\title{
Thermal Management at Hypersonic Leading Edges
}

\author{
A Dissertation \\ Presented to \\ The Faculty of the School of Engineering and Applied Science \\ University of Virginia
}

in partial fulfillment

of the requirements for the degree in

Doctor of Philosophy

Scott D. Kasen

May 2013 


\section{APPROVAL SHEET}

This dissertation is submitted in partial fulfillment of the requirements for the degree of

Doctor of Philosophy

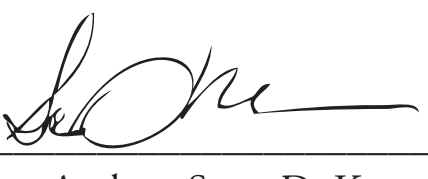

Author, Scott D. Kasen

This dissertation has been read and approved by the examining committee:
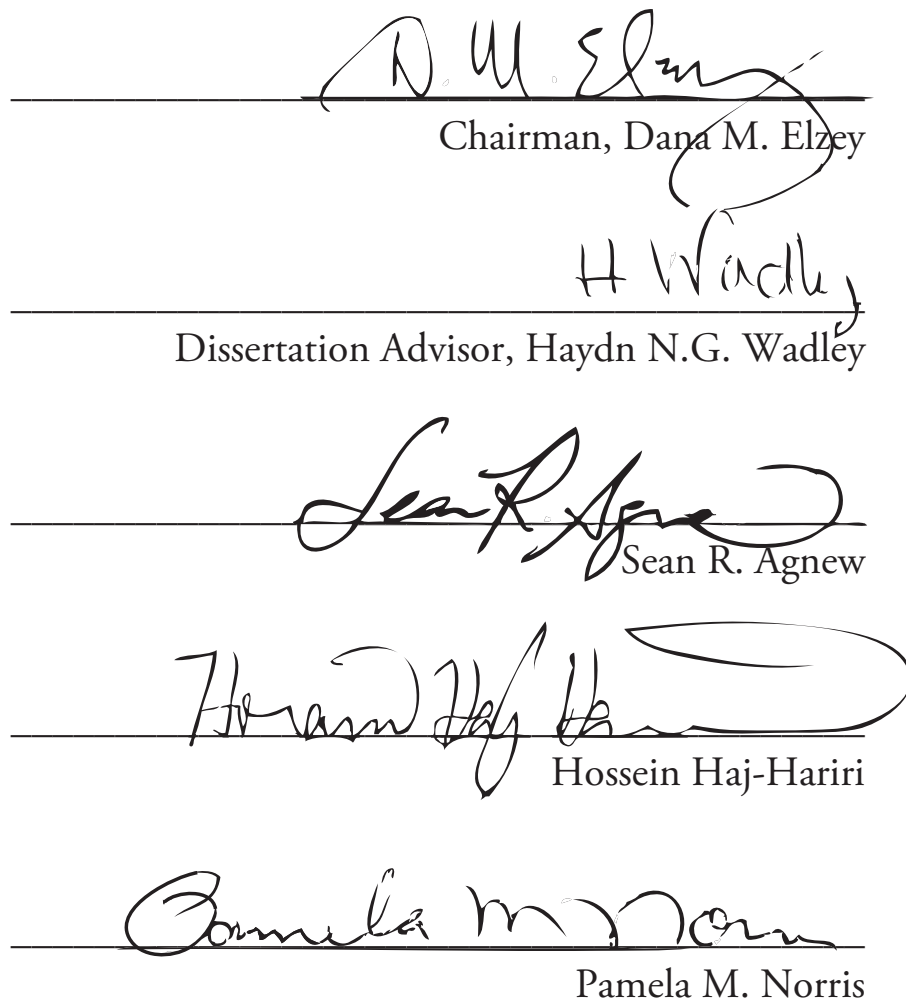

Accepted for the School of Engineering and Applied Science:

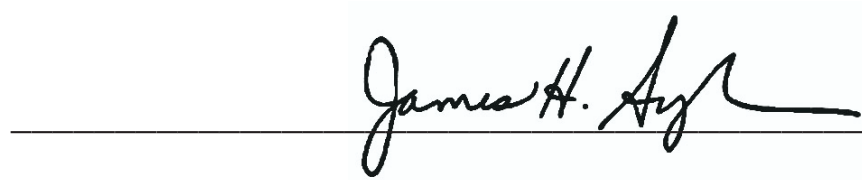

Dean, School of Engineering and Applied Science 


\section{Abstract}

The intense heat flux incident upon the leading edges of hypersonic vehicles traveling through the low earth atmosphere at speeds of Mach 5 and above requires creative thermal management strategies to prevent damage to leading edge components. Conventional thermal protection systems (TPSs) include the ablative coatings of NASA's Mercury, Gemini, and Apollo vehicles and the reusable reinforced carbon-carbon (RCC) system of the Space Shuttle Orbiter. The ablative approach absorbs heat by endothermic transformation (phase and/or chemical change to the polymeric coating). The heat is dissipated from the vehicle as the single-use coating eventually vaporizes. The RCC approach manages the intense heat by operating at high temperatures and radiating heat to its surroundings. The effectiveness of both approaches is predicated on keeping the heat flux that impinges upon the susceptible aluminum airframe below a critical level. 
This dissertation has explored an alternative metallic TPS concept which seeks to redistribute the heat from the leading edge, thereby eliminating local hot spots. It makes use of high thermal conductance heat pipes coupled to the leading edge so that the thermal load may be redistributed from a high heat flux location (at the stagnation point) to regions where it can be effectively radiated from the vehicle. The sealed system concept is based upon the evaporation of a fluid near the heat source that sets up a region of elevated vapor pressure inside the pipe. The latent heat is transported down the resulting pressure gradient by the vapor stream where it condenses at cooler regions, releasing the heat for removal. Replenishment of the condensed working fluid to the evaporator region is accomplished through the capillary pumping action of a porous wick which lines the interior surface of the pipe.

A design methodology for a wedge-shaped heat pipe is presented which uses a coupled flow-wall temperature model to construct design maps which relate design parameters of the leading edge system (overall length, wall thickness, and alloy) to its operating conditions (isothermal temperature, maximum temperature, maximum thermal stress). Potential bounds on heat transport due to physical phenomena linked to the sound speed within a chamber (sonic limit), capillarity, and boiling nucleation are considered by extending models developed for tube designs to the wedge geometry. A new heat flux limit is proposed which, should it be exceeded, subjects the leading edge to thermally-induced plastic deformation of the TPS. 
To investigate the validity of the design approach and thermal spreading effectiveness of the proposed concept, a low temperature wedge-shaped leading edge was designed and constructed using stainless steel as the case material and water as the working fluid. Under localized tip heating, the maximum temperatures were significantly reduced compared to an otherwise identical but evacuated (no working fluid) test article. Isothermal operation was observed over its length. There was good agreement between experimental and design predictions. To test the concept at hypersonic flow enthalpies and temperatures, a high temperature Ni-based Inconel / sodium system was designed, fabricated, and tested. While there was a significant reduction in maximum temperature over an identical system containing no working fluid, isothermal operation was not observed. It is hypothesized that there is a lower bound on the wall heat flux which must be exceeded for the evaporated fluid to behave in the continuum flow regime predicted by the models. Finally, an assessment is made on three material-working fluid system combinations (Inconel/sodium, Nb-based C103/lithium, and Mo-based TZM/lithium) for a leading edge TPS that would be utilized by air-breathing hypersonic vehicles of the future. 


\section{Acknowledgements}

When I embarked on this journey, I thought unbridled enthusiasm, curiosity and hard work would guarantee success. However, it was not without its stumbling blocks. Had I not been surrounded by love and support, this journey would not have been completed.

I would like to thank my advisor, Haydn Wadley, who pushed me to explore outside my academic "comfort zone" and from who I have tried to learn the art of elegant research.

I am appreciative of my advising committee for their time and effort; in particular, Dana Elzey, whose mentorship during my early graduate years was invaluable to the completion of this work, and Hossein Haj-Hariri, who probably doesn't realize the value gained by me through his constant encouragement and reassurance. 
I would also like to acknowledge the support of my IPM labmates and work colleagues, especially Doug Queheillalt who offered his support at the University and afterwards. Thanks also to Tommy Eanes, and Sherri Sullivan, who each spent many hours supporting my work, and to David Glover, whose machining and "anything-else-you-need" skills (even an open ear to listen) are unmatched. A special thank you also to Don Jordan for his helpful discussions.

Finally, I would also like to acknowledge:

Sammy, who always gives me a greeting when I arrive home.

My parents, brother, and in-laws, who have given an enormous amount of their time, love, and support to this endeavor. Thank you so much.

My sons, Jacob and Jon, whose smiling faces and unconditional love remind me why I followed this journey to the end. I am so proud of you both.

And my wife Sarah, who has sacrificed more than anyone (me included) so that I could complete this work. She has given me the time, support, encouragement, proofreading, hope, pencils and paper, packed lunches, help, late night snacks, completed errands, filed taxes, inspiration...thank you for everything. I love you. 


\section{Table of contents}

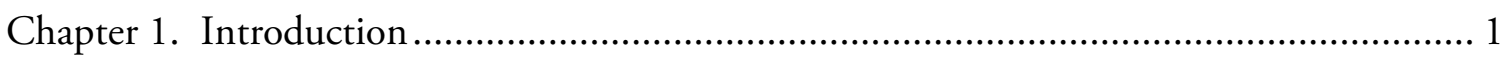

1.1. Rationale for dissertation.............................................................................. 1

1.2. Dissertation objectives................................................................................... 9

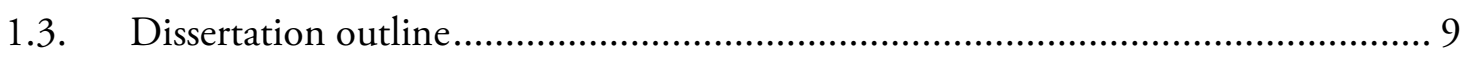

Chapter 2. Thermal protection concepts ......................................................................... 11

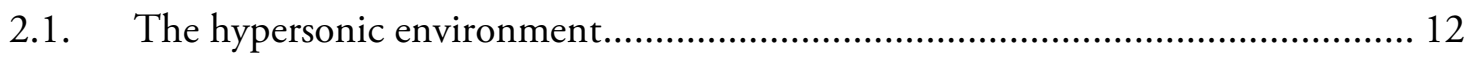

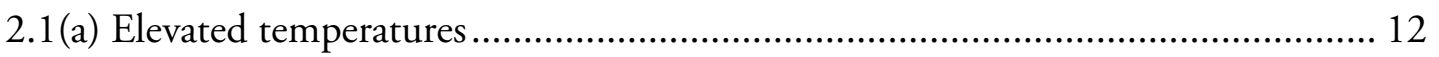

2.1(b) Thermal gradients and stresses...................................................................... 14

2.1(c) Chemically reacting flows .......................................................................... 19

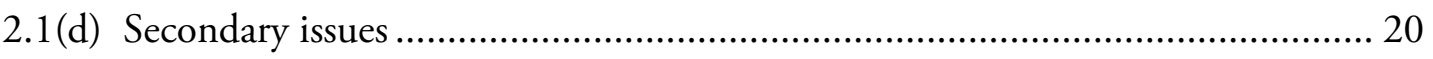

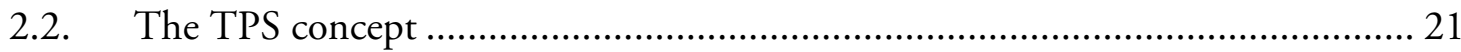

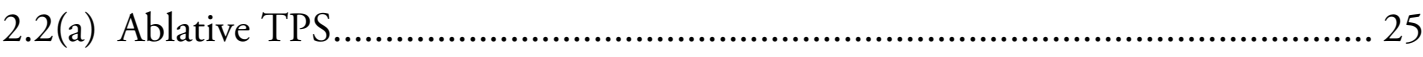

2.2(b) Hot structure concepts ................................................................................... 29

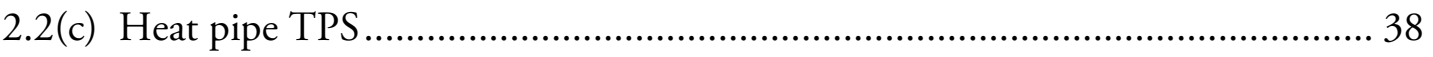

2.3. Proposed leading edge heat spreader ................................................................. 47

Chapter 3. Leading edge design methodology using hypersonic flow theory ....................... 51 


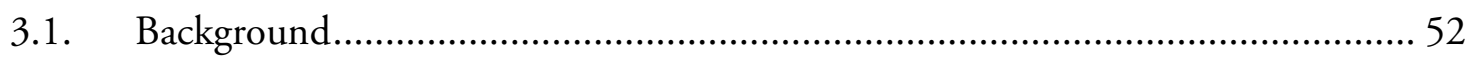

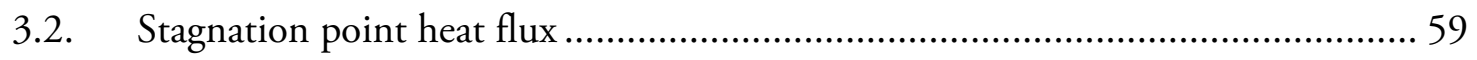

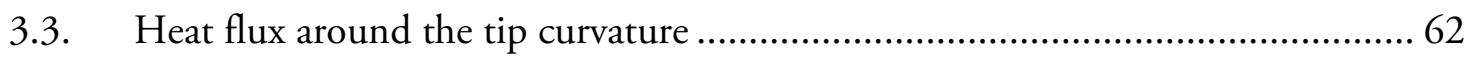

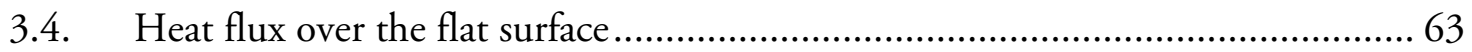

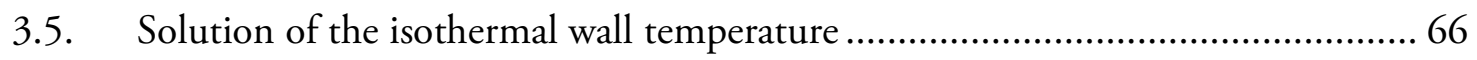

Solution of the maximum temperature and thermal stress ............................................ 70

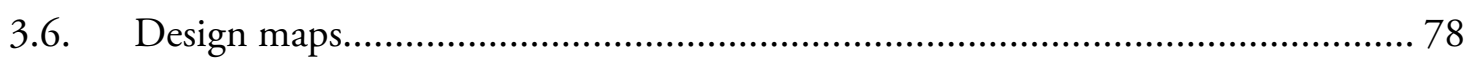

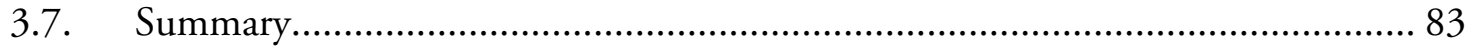

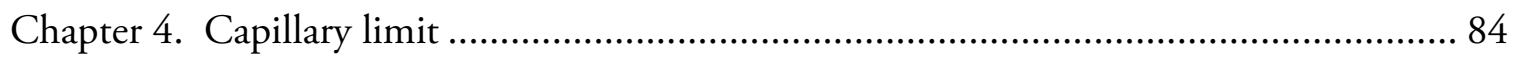

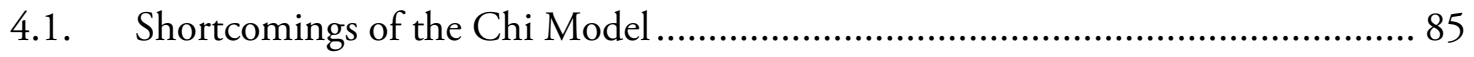

4.2. Surface tension and the liquid-vapor interface .................................................... 95

4.3. Modified Chi Model (Cylindrical Pipe) ………............................................. 103

4.3(a) Assumptions ........................................................................................... 103

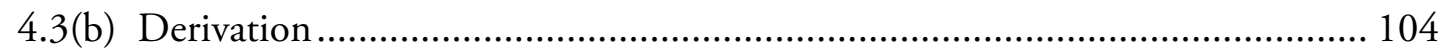

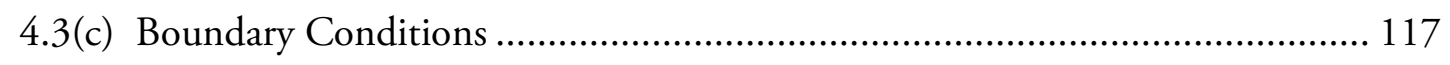

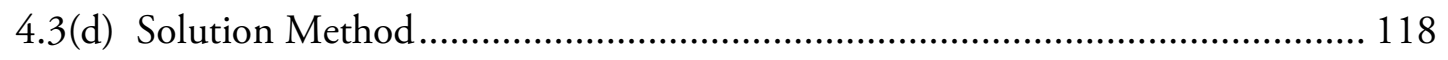

4.3(e) Results and discussion.................................................................................. 121

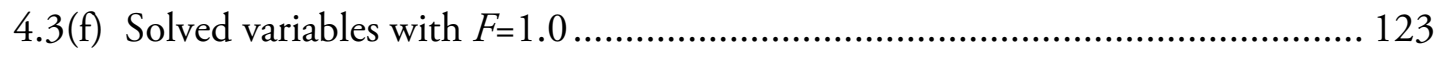

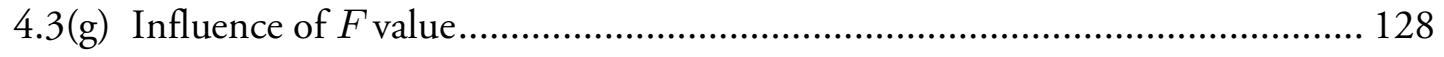

4.3(h) Validation of the Modified Chi Model............................................................. 130

4.4. Modified Chi Model (Wedge Geometry) ......................................................... 135

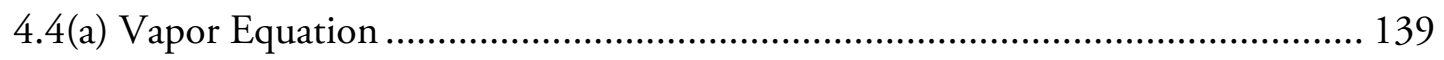

4.3(b) Wall/Wick and Wick/Vapor Heat Balance ................................................... 140

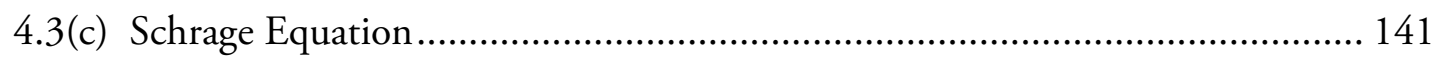

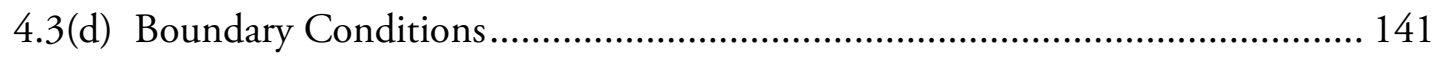

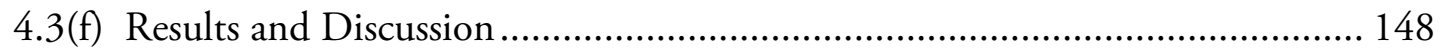

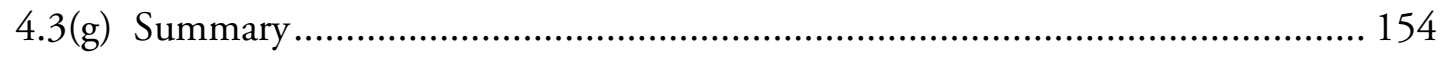

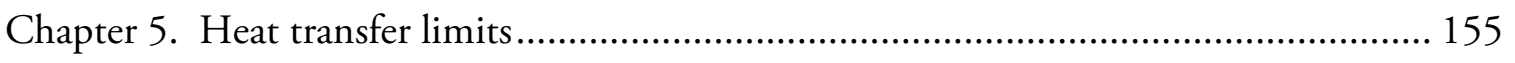

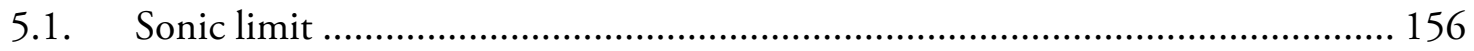




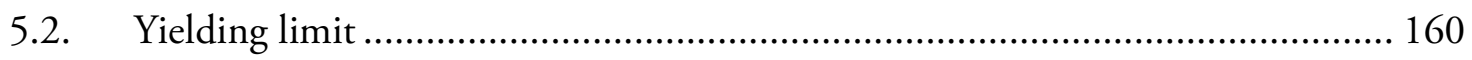

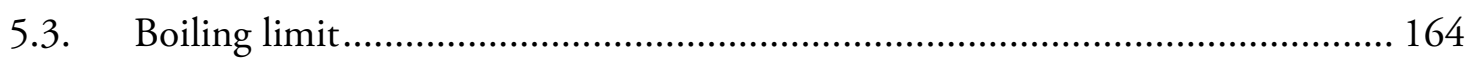

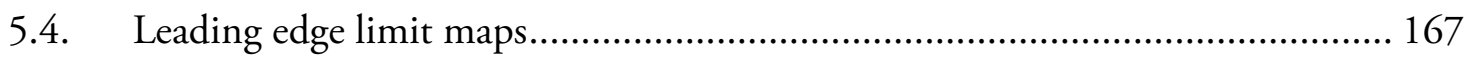

Chapter 6. Low temperature evaluation...................................................................... 173

6.1. Leading edge design and fabrication .......................................................... 174

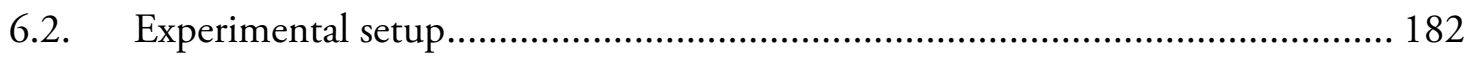

6.3. Experimental results ......................................................................... 184

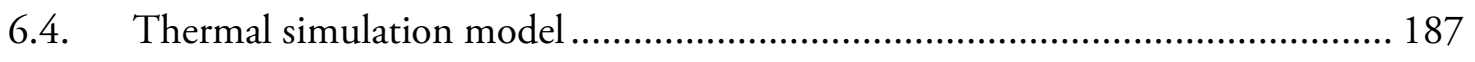

6.4 (a) Vapor Thermal Conductivity...................................................................... 188

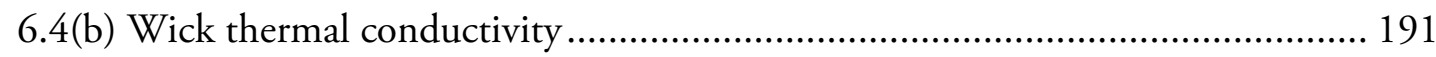

7.4(c) Boundary Conditions ............................................................................... 192

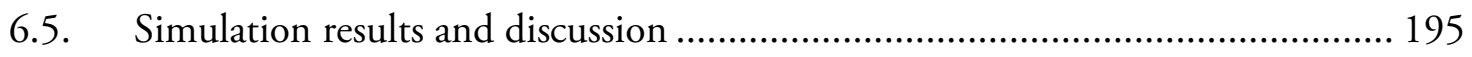

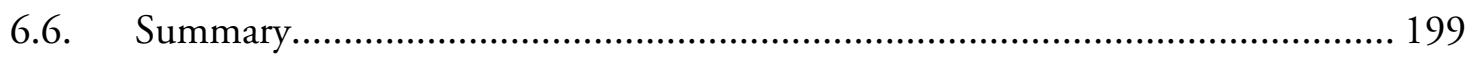

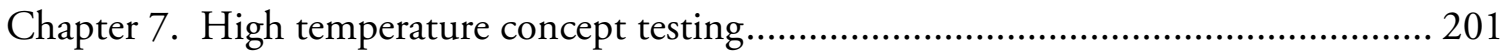

7.1. Leading edge design and fabrication ............................................................. 202

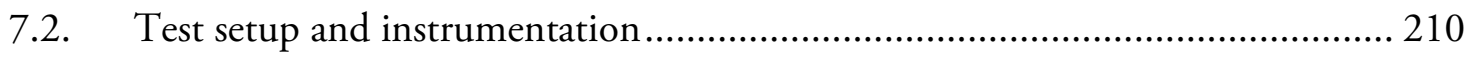

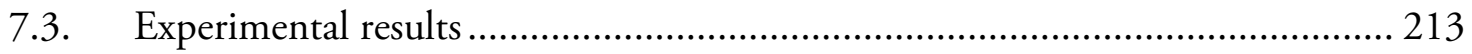

7.4. Finite element model ................................................................................. 216

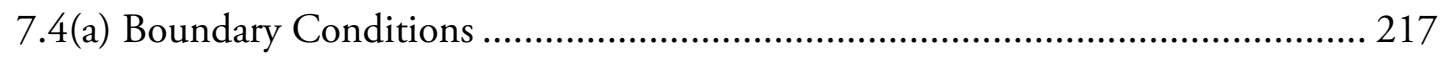

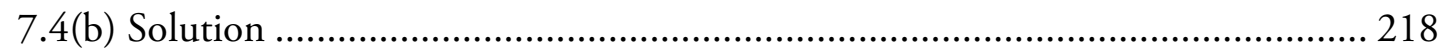

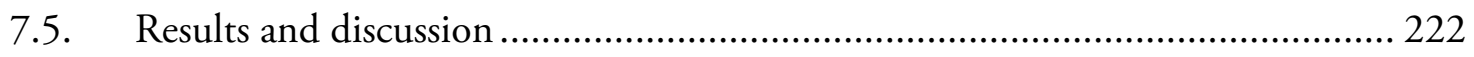

Chapter 8. Discussion ............................................................................................ 229

8.1. Two-model design methodology ………………......................................... 229

8.2. Assessment of metallic leading edges for hypersonic flight ................................ 239

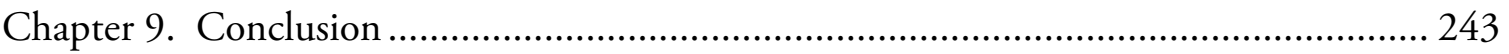

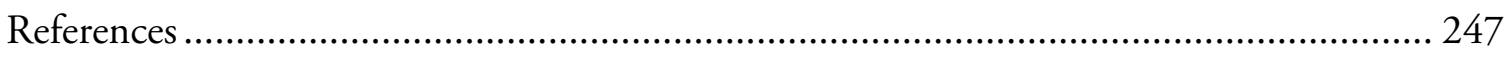

Appendix A. Thermo-mechanical properties for SS-304, IN718,C-103, and TZM......... 259

Appendix B. Working fluid properties for water, sodium, and lithium............................ 270

Appendix C. Derivation of the vapor momentum equation (cylindrical pipe) .................. 274 
Appendix D. Derivation of the vapor momentum equation (wedge geometry) ............... 278

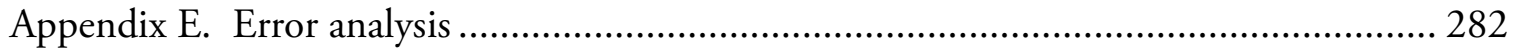




\section{List of figures}

Figure 1.1. Thermodynamic properties of air as a function of altitude based on the U.S. Standard Atmosphere [4].

Figure 1.2. Schematic illustration of the DARPA/ONR hypersonic HyFly vehicle showing areas of stagnation point heating at the nose cone, scramjet inlet, and tail fin leading edges. 5

Figure 1.3. (a) The relationship between altitude and Mach number for a dynamic pressure

of $40 \mathrm{kPa}$. (b) The influence of leading edge radius on the stagnation point heat flux assuming a cold wall (the temperature of the gas >> temperature of the vehicle).................... 7

Figure 2.1. Material property chart of yield strength normalized by density and maximum service temperature for common metals and aerospace composites.

Figure 2.2. (a) Leading edge geometry defined by tip radius $R_{L E}$ and half angle $\varphi$, and (b) the heat flux profile along $x$ for a wedge with $R_{L E}=3 \mathrm{~mm}$ and $\varphi=6^{\circ}$ at Mach 7 $(29.0 \mathrm{~km})$.

Figure 2.3. Chart showing the relative potential of materials for localized high heat flux, high temperature environments. The most favorable materials are ones with high yield strength, high thermal conductivity, low stiffness, and low thermal expansion, which can be 
found in the upper left quadrant. There is a material gap between diamond and the refractory metallics. 18

Figure 2.4. Velocity-altitude map showing the flight condition at which vibrational excitation and chemical reactivity effects of the gas are significant. Most airbreathing flight trajectories are subjected to significant vibrational excitation effects only. Altitude-velocity map from [3]. Iso-Mach lines (....) have been added for reference. Flight data compiled from [3], [7], [20], [21], [22].

Figure 2.5. Various TPS concepts in operation or proposed for use on hypersonic vehicles. Adapted and modified from [26]. 22

Figure 2.6. The heat dissipation mechanisms of ablation heat shields. Adapted from [31] and [39]. 26

Figure 2.7. The specific yield strength of some high temperature metals and ceramics. ..... 31

Figure 2.8. TPS for NASA's X-43A experimental vehicle. Adapted from [32] 33

Figure 2.9. Advanced RCC with oxidation protection coating [53].... 34

Figure 2.10. Crack in a RCC Shuttle leading edge from a laboratory-controlled foam strike [51]. 38

Figure 2.11. Cross-section schematic of a cylindrical heat pipe illustrating its operation.... 40

Figure 2.12. (a) An Orbiter leading edge developed by NASA using a Hastelloy X and sodium working fluid [33], and (b) a Mo-Re heat pipe embedded in a RCC leading edge skin [35]..... 41

Figure 2.13. Examples of periodic cellular materials. From [78]. 44

Figure 2.14. Leading edge I-core system which requires bending to form the leading edge tip radius [88]. 46

Figure 2.15. Three leading edge heat spreader concepts: (a) an I-core design having low flow resistance in the longitudinal direction, (b) a perforated corrugation design and (b) cruciform (truncated honeycomb) design, both of which allow longitudinal and transverse vapor flow. 48 
Figure 2.16. Thermal operating window of some heat pipe working fluids [72]. 50

Figure 3.1. Local balance of the heat fluxes at a leading edge surface. The positive flux direction is into the vehicle and " $s$ " is a coordinate on the leading edge surface. 53

Figure 3.2. Shock pattern and flow variables around the leading edge. Adapted from [3]. 56

Figure 3.3. The heat flux incident upon an IN718 leading edge for $M_{\infty}=2$ to 6 at the solved isothermal wall temperatures. $R_{L E}=3 \mathrm{~mm}, \varphi=6^{\circ}, L_{\text {flat }}=82.3 \mathrm{~mm}$.

Figure 3.4. Isothermal wall temperatures for (a) IN718, (b) C-103, and (c) TZM alloys as a function of length for free-stream Mach numbers 5-12. $R_{L E}=3 \mathrm{~mm}$ and $\varphi=6^{\circ}$.

Figure 3.5. Schematic illustration of thermal stress state at the leading edge tip. (a) Unconstrained material showing strain misfit due to differential thermal expansion. (b) Localized heating generates a through-thickness stress gradient which causes failure when the yield strength is exceeded. 72

Figure 3.6. Enlarged view of the leading edge. 73

Figure 3.7. Effects of the wall thickness and design length upon $T_{\max }$ for IN718 alloy leading edge in (a) Mach $5(24.5 \mathrm{~km})$ and (b) Mach $6(26.9 \mathrm{~km})$ flows. $R_{L E}=3 \mathrm{~mm}$ and $\varphi=6^{\circ}$ 76

Figure 3.8. Effects of the wall thickness and design length upon $T_{\max }$ for C-103 alloy leading edge in (a) Mach $6(26.9 \mathrm{~km})$ and (b) Mach $8(30.8 \mathrm{~km})$ flows. $R_{L E}=3 \mathrm{~mm}$ and $\varphi=6^{\circ}$ 76

Figure 3.9. Effects of the wall thickness and design length upon $T_{\max }$ for TZM alloy leading edge in (a) Mach $6(26.9 \mathrm{~km})$ and (b) Mach $8(30.8 \mathrm{~km})$ flows. $R_{L E}=3 \mathrm{~mm}$ and $\varphi=6^{\circ}$ 77

Figure 3.10. IN718 alloy design maps for a leading edge operating at (a) Mach 5 and (b) Mach 6. 80

Figure 3.11. C-103 alloy design maps for a leading edge operating at (a) Mach 6 and (b) Mach 8 
Figure 3.12. TZM alloy design maps for a leading edge operating at (a) Mach 6 and (b) Mach 8

Figure 4.1. Cross-section view of a cylindrical heat pipe as represented by the Chi model. The evaporation/condensation cycle is shown in (a), with close-ups of the evaporator meniscus profile in (b) and condenser meniscus profile in (c). 87

Figure 4.2. Pressure profiles for an operating heat pipe. Adapted from [73]. 89

Figure 4.3. Molecular view of a liquid-vapor interface as represented by (a) kinetic theory and (b) the Chi model. The Chi model omits the interfacial region which is the active site for evaporation and condensation processes.

Figure 4.4. Liquid meniscus profile showing the variation in heat flux across a wick pore. Evaporation is suppressed at a small film thickness due to intermolecular attraction between the solid and liquid. It is likewise suppressed at the central meniscus which has a long thermal conduction pathway. 95

Figure 4.5. Schematic representation of (a) the intermolecular potential and (b) force as it depends on separation distance between two isolated molecules. The derivative of the potential gives the force. Adapted from [119]

Figure 4.6. View of (a) the liquid-vapor interface showing three distinct regions whose fluid density is schematically plotted in (b). Near the liquid surface, forces of attraction $(\longrightarrow$ ) and repulsion $(-->)$ as a function of depth are shown in (c). Adapted from [108], [118].

Figure 4.7. Free body diagram of a pore's liquid meniscus. 102

Figure 4.8. The heat pipe used for the modified Chi model is shown in (a), with close-up views of the (b) evaporator meniscus and (c) condenser meniscus. 106

Figure 4.9. Force balance for an interfacial molecule at a (a) flat and (b) curved interface. An increase in intermolecular spacing above the curved surface maintains the force balance. 108

Figure 4.10. Schematic of the meniscus interface. An elevated view in (a) shows the repeating unit cell pore with area $A_{C}$. The wick's unit cell, shown in (b), has evaporation occurring with local mass flux $\dot{m}_{i}$ over the thin film region of projected area $A_{A}$. The local 
mass flux $\dot{m}_{i}$ (mass per unit time per unit area) can be represented as a unit cell mass flux $\bar{m}_{i}$ over $A_{C}$ with an equivalent evaporation rate (mass per unit time). 113

Figure 4.11. Applied cumulative heat flow through the length of the heat pipe. 118

Figure 4.12. Flow diagram for the solution algorithm. 121

Figure 4.13. Calculated vapor and liquid pressure drops using the modified Chi model for the sodium heat pipe described by and operating at $Q_{\max }=21.6 \mathrm{~kW}$ with $T_{V, o}=927^{\circ} .124$

Figure 4.14. The vapor mach number as a function of position using the modified Chi model for the sodium heat pipe operating at $Q_{\max }=21.6 \mathrm{~kW}\left(T_{V, o}=927^{\circ} \mathrm{C}\right)$.........

Figure 4.15. The vapor density as a function of position using the modified Chi model for the sodium heat pipe operating at $Q_{\max }=21.6 \mathrm{~kW}\left(T_{V, o}=927^{\circ} \mathrm{C}\right) \ldots \ldots \ldots \ldots \ldots \ldots \ldots \ldots \ldots \ldots . . . .126$

Figure 4.16. The meniscus radius as a function of position using the modified Chi model for the sodium heat pipe operating at $Q_{\max }=21.6 \mathrm{~kW}\left(T_{V, o}=927^{\circ} \mathrm{C}\right)$. A sketch of the liquid surface profile along the pipe is provided above the plot.

Figure 4.17. Temperature profiles generated by the modified Chi model for the sodium heat pipe operating at $Q_{\max }=21.6 \mathrm{~kW}\left(T_{V, o}=927^{\circ} \mathrm{C}\right)$. 128

Figure 4.18. Comparison of the pressure drops for the modified Chi model using active interfacial mass transfer regions of $100 \%$ and $10 \%$ (i.e., $F=1.0$ and $F=0.1$, respectively). The wet point has shifted towards the middle of the condensation zone for the lower value of F..

Figure 4.19. Axial wall temperature profile, $T_{W}$, for several $F$ values.

Figure 4.20. Limits map for heat pipe performance. Experimental data, viscous, and sonic limits reproduced from [104]. Each data symbol type represents changes made to the experimental setup to try and improve the experimentally determined limits in the capillary limit region.

Figure 4.21. Geometry of the wetted unit cell: (a) elevated view, (b) profile view. 134

Figure 4.22. Evaporation mass flux as a function of distance along a microchannel wall of varying widths and with a wall superheat of $1 \mathrm{~K}$. The evaporating film length is nearly 
independent of microchannel width. Significant evaporation occurs within the first 2 to $4 \mu \mathrm{m}$. Reproduced from [123].

Figure 4.23. (a) Leading edge thermal spreader with wedge geometry. The real system in (a) is modeled as the diverging duct in (b). 138

Figure 4.24. Heat profiles used as thermal boundary conditions in the model. In (a), the simplified tanh function for the heat flux through the wall compares well to the full hypersonic theory. The cumulative power of the system is shown to obey an energy balance using the tanh approximation in (b). $R_{L E}=3 \mathrm{~mm}$ and $\varphi=6^{\circ}$ 144

Figure 4.25. Vapor Mach number as a function of axial position for the Sodium-IN718 leading edge operating at its capillary limit of $q_{\text {cap }}=217.1 \mathrm{~kW} / \mathrm{m}^{2}$.

Figure 4.26. Pressure profiles as a function of axial position for the Sodium-IN718 leading edge operating at its capillary limit of $q_{\text {cap }}=217.1 \mathrm{~kW} / \mathrm{m}^{2}$ 150

Figure 4.27. Capillary pressure (left ordinate) and meniscus radius (right ordinate) as a function of axial position for the Sodium-IN718 leading edge operating at its capillary limit of $q_{\text {cap }}=217.1 \mathrm{~kW} / \mathrm{m}^{2}$.

Figure 4.28. Axial temperature profiles for the Sodium-IN718 leading edge operating at its capillary limit of $q_{c a p}=217.1 \mathrm{~kW} / \mathrm{m}^{2}$

Figure 4.29. Parameter map for $L_{\text {flat }}$ and $b_{\text {wall }}$ showing iso-flux lines for $q_{\text {cap }} \ldots \ldots \ldots \ldots . . . .154$

Figure 5.1. The maximum heat flux subject to the sonic limit using sodium and lithium as working fluids.

Figure 5.2. Influence of wall thickness and vapor stagnation temperature (at $x=0$ ) on the yielding limit for a (a) IN718, (b) C-103, and (c) TZM case material. 163

Figure 5.3. Nucleate boiling in the wick at the leading edge tip leading to a dryout condition. 165

Figure 5.4. The maximum heat flux subject to wick boiling plotted as a function of wick thickness for a sodium-saturated nickel wick at $827^{\circ} \mathrm{C}$ and lithium-saturated C-103 and TZM wicks at $1,227^{\circ} \mathrm{C}$. $R_{L E}=3 \mathrm{~mm}$. 167 
Figure 5.5. Operational map for the sodium-IN718 system. 170

Figure 5.6. Operational map for the lithium-C103 system. 171

Figure 5.7. Operational map for the lithium-TZM system. 172

Figure 6.1. Friction factors for various core topologies as a function of the Reynolds number. The lightened diagonal lines indicate contours of constant ( $\left.\mathrm{f} \mathrm{Re}_{\mathrm{V}}\right)$. Adapted from [129]. 175

Figure 6.2. Schematic of the I-core leading edge design. 176

Figure 6.3. Photograph of the stainless steel leading edge system showing (a) the curved leading edge and (b) the internal core and wick (prior to assembly of the rear face sheet). 178

Figure 6.4. Evacuation and charging apparatus connected to the test article. 181

Figure 6.5. Experimental setup for testing the low temperature leading edge heat spreader. 184

Figure 6.6. Steady state, top surface thermographs of (I) the evacuated and (II) charged leading edge at several applied power levels. 186

Figure 6.7. The effective thermal conductivity of the vapor as a function of $Q_{\max }$. The right ordinate normalizes $k_{\text {eff }}$ by the room temperature thermal conductivity of copper $\left(k_{C u} \approx 400 \mathrm{~W} / \mathrm{mK}\right)$.

Figure 6.8. Boundary conditions of the quarter symmetry model. 193

Figure 6.9. Isometric view of quarter-symmetry model showing the mesh. 194

Figure 6.10. Comparison of the simulated and experimental line profile surface measurements of (I) the evacuated and (II) charged leading edge at applied power levels of 5, 15 , and $25 \mathrm{~W}$. The inset in I(a) shows the location of the line profile. The "•" data point is the tip thermocouple temperature reading. 196

Figure 6.11. Steady state comparison of the predicted surface temperature difference to experimental data. The difference is taken from the thermocouple measurement at the tip to 
the rear edge IR measurement as shown in the inset. The dashed lines are best fits to the data. 197

Figure 6.12. Operating limits for the water-SS304 leading edge heat spreader. The sonic and boiling, and yielding limits are not shown because they are well above the plot's range. 198

Figure 6.13. Comparison of isothermal surface temperature (taken by the IR camera) and vapor pressure measurements correlated by the Clausius-Clapeyron equation. The IR temperature measurements correspond to the right ordinate.

Figure 7.1. Schematic of the IN718 / $\mathrm{Na}$ system with relevant design parameters. Aerodynamic constraints set $R_{L E}=3 \mathrm{~mm}$ and $\varphi=6^{\circ}$

Figure 7.2. IN718 / Na design map for Mach 5 flight.

Figure 7.3. Fabrication and assembly process for the high temperature leading edge using the solid-Na charging procedure. 208

Figure 7.4. Scanning electron micrograph of the sintered foam-IN718 interface.

Figure 7.5. Photograph of the fabricated high temperature leading edge (a) prior to insertion of the wick and (b) after diffusion bonding of the wick. The completed test article is shown in $(\mathrm{c})$.

Figure 7.6. (a) Setup for the high heat flux experiments, and (b) photograph of the sample tip during testing.

Figure 7.7. Plots comparing the steady state surface temperature profiles of the empty and charged systems at three standoff positions: (a) $175 \mathrm{~mm}$, (b) $158 \mathrm{~mm}$, and (c) $146 \mathrm{~mm}$....... 214

Figure 7.8. Maximum $\Delta T_{s}$ taken over the sample's length as shown in the inset. The dashed lines are a best-fit approximation.

Figure 7.1. Two views $[(a)$ and (b)] of the finite element mesh superimposed on the solid model. 
Figure 7.2. Isometric view of quarter-symmetry charged model showing the applied boundary conditions. The evacuated model is identical but has no vapor core and its wick interior surface is treated adiabatic. 218

Figure 7.9. Steady state thermal profile plots of (I) the iterative FE solution and experiment for the evacuated sample which confirmed the boundary conditions of the model, and (II) a comparison of the simulation and experiment for the charged sample. 220

Figure 7.10. Axial temperature profiles of the charged system for seven standoff positions. There is the emergence of an inflection point at position $434.3 \mathrm{~kW} / \mathrm{m}^{2}$, which persists through tests at $563.8 \mathrm{~kW} / \mathrm{m}^{2}$ and $671.2 \mathrm{~kW} / \mathrm{m}^{2}$. Error is $\pm 2 \%$ of the temperature reading; errors bars have been removed for clarity.

Figure 7.11. Illustration showing the vapor core transition from a rarefied gas, to a continuum vapor, to a dryout condition and each operating regime's representative temperature profile. 226

Figure 7.12. Sodium-IN718 limits map showing the regions probed by the experimental work. The vapor temperature is estimated to be equivalent to the surface temperature immediately aft of the ceramic shield. The shaded area indicates a region made nonoperational by the Knudsen limit (for $D=2 H_{V}(x=0)$ ).

Figure 8.1. Design map for the SS304 wedge subjected to $25 \mathrm{~W}$ over its tip curvature. The yield strength-temperature curve for SS304 is well above the plot's range.

Figure 8.2. Heat transport limits map for the water-SS304 leading edge heat spreader. The sonic, boiling, and yielding limits are not shown because they are well above the plot's range. 234

Figure 8.3. Design map for the tested IN718-sodium leading edge under Mach $5(24.5 \mathrm{~km})$ enthalpy conditions.

Figure 8.4. Heat transport limits map for the IN718-sodium system with the proposed Knudsen limit. There is no operational window (indicated by a fully shaded plot). 236

Figure 8.5. Heat transport limits map for the sodium-IN718 system showing the dependence of the Knudsen limit (for $D=2 H_{V}(x=0)$ ) and flight operating condition on the design length, $L_{\text {flat }}$. There is no operational window (indicated by a fully shaded plot). 
Figure 8.6. Heat transport limits map for an IN718/sodium system showing the dependence of the capillary limit on the wick pore size. The white area delineates the

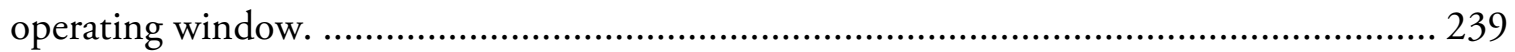

Figure 8.7. Heat transport limits map for a C103/lithium system. The white area delineates

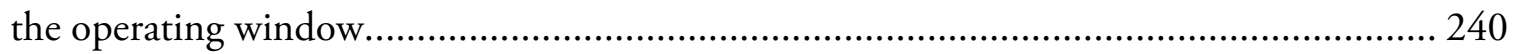

Figure 8.8. Heat transport limits map for a TZM/lithium system. The white area delineates

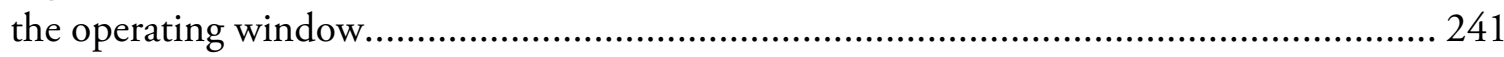

Figure A.1 Heat capacity temperature dependence. …........................................... 261

Figure A.2 Yield strength temperature dependence.................................................... 263

Figure A.3 Young's modulus temperature dependence. ............................................ 265

Figure A.4 Thermal conductivity temperature dependence. ..................................... 267

Figure C.1. Control volume for the vapor momentum equation of a constant area duct. 274

Figure D.1. Control volume for the vapor momentum equation of a diverging duct....... 278 


\section{List of tables}

Table 3.1. Calculated free-stream and stagnation conditions for Mach 5-12 flight at a dynamic pressure $(\rho \infty V \infty 2 / 2)$ of $48 \mathrm{kPa}[96]$.

Table 4.1. The nine equation system and variables for the modified Chi model of the cylindrical heat pipe.

Table 4.2. Relevant parameters for the Vinz and Busse heat pipe [104]

123

Table 4.3. List of the system of nine equations and unknown variables for the leading edge heat pipe. 146

Table 4.4. Relevant parameters for the sodium-IN718 Alloy leading edge system. 148

Table 5.1. Relevant parameters for the high temperature leading edge systems. 168

Table 6.1. Relevant parameters for the low temperature leading edge heat pipe. 179

Table 7.1. Relevant parameters for the high temperature leading edge heat spreader. 205 
Table 8.1. Influence of design parameters and material selection on limits for a leading edge

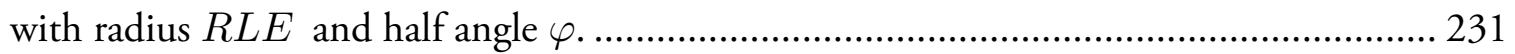

Table 8.2. Design values for hypersonic leading edges of three case alloy/working fluid

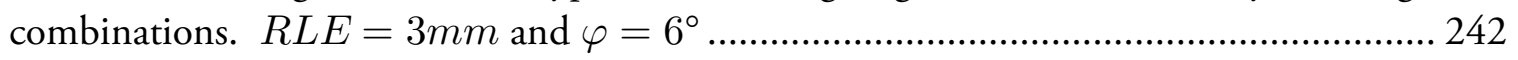

Table A. 1. Temperature dependent yield strength of IN718 [140] ............................. 264

Table A. 2. Temperature dependent yield strength of C103 [139] ............................... 264

Table A. 3. Temperature dependent yield strength of TZM [28] ................................. 264

Table E.1. Infrared temperature uncertainty............................................................ 283

Table E.2. Uncertainties associated with the applied power....................................... 284

Table E.3. Uncertainties associated with the temperature difference calculation............. 285

Table E.4 Uncertainties associated with the thermal stress calculation........................... 286 


\title{
List of symbols
}

\author{
$A_{A} \quad$ surface area of unit cell active in evaporation in the wick \\ $A_{C} \quad$ surface area of unit cell in the wick \\ $A_{V} \quad$ vapor space cross-sectional area \\ $A_{c} \quad$ surface area of curved tip region \\ $A_{\text {wick }} \quad$ wick cross-sectional area \\ $\bar{A}_{V} \quad$ average vapor space cross-sectional area \\ Bi Biot modulus \\ $b_{\text {wall } \quad \text { case wall thickness }}$ \\ $b_{\text {wick }} \quad$ wick thickness \\ $C_{f}^{*} \quad$ reference friction factor \\ $C_{H} \quad$ Stanton Number \\ $C_{p, a w} \quad$ specific heat capacity of the wall material at its adiabatic temperature \\ $C_{p, w} \quad$ specific heat capacity of the wall material \\ $C_{p, \infty} \quad$ free-stream specific heat capacity at constant pressure \\ c speed of sound, accomodation coefficient \\ $c_{p} \quad$ specific heat capacity at constant pressure
}




\begin{tabular}{|c|c|}
\hline$D$ & characteristic dimension \\
\hline$d_{w}$ & wire diameter \\
\hline$E$ & Young's modulus \\
\hline$e$ & thermal effusivity \\
\hline$F$ & areal fraction of wick unit cell surface active in evaporation \\
\hline$F_{\max }$ & maximum areal fraction of wick unit cell surface available for evaporation \\
\hline$F_{L}$ & force vector acting liquid \\
\hline$F_{L V-E Q}$ & force vector acting on interfacial region (due to $P_{L V-E Q}$ ) \\
\hline$F_{C}$ & force vector due to Laplace pressure \\
\hline$f_{c}$ & compressible friction factor \\
\hline$f_{V}$ & duct friction factor \\
\hline$H_{V}$ & vapor space height \\
\hline$h$ & convective heat transfer coefficient \\
\hline$h_{\text {cool }}$ & natural convection coefficient \\
\hline$h_{e, a w}$ & adiabatic wall enthalpy behind the oblique shock \\
\hline$h_{s t, a w}$ & adiabatic wall enthalpy at the stagnation point \\
\hline$h_{w}$ & wall enthalpy \\
\hline$h_{\infty}$ & free-stream enthalpy \\
\hline$K$ & wick permeability \\
\hline $\mathrm{Kn}$ & Knudsen number \\
\hline$k_{C u}$ & thermal conductivity of copper \\
\hline$k_{e f f}$ & effective thermal conductivity \\
\hline$k_{L}$ & liquid thermal conductivity \\
\hline$k_{S}$ & thermal conductivity of the solid wick material \\
\hline$k_{\text {wall }}$ & wall material thermal conductivity \\
\hline$k_{w i c k}$ & effective wick thermal conductivity \\
\hline$L_{a}$ & adiabatic zone length \\
\hline$L_{c}$ & condenser length \\
\hline$L_{e}$ & evaporator length \\
\hline
\end{tabular}




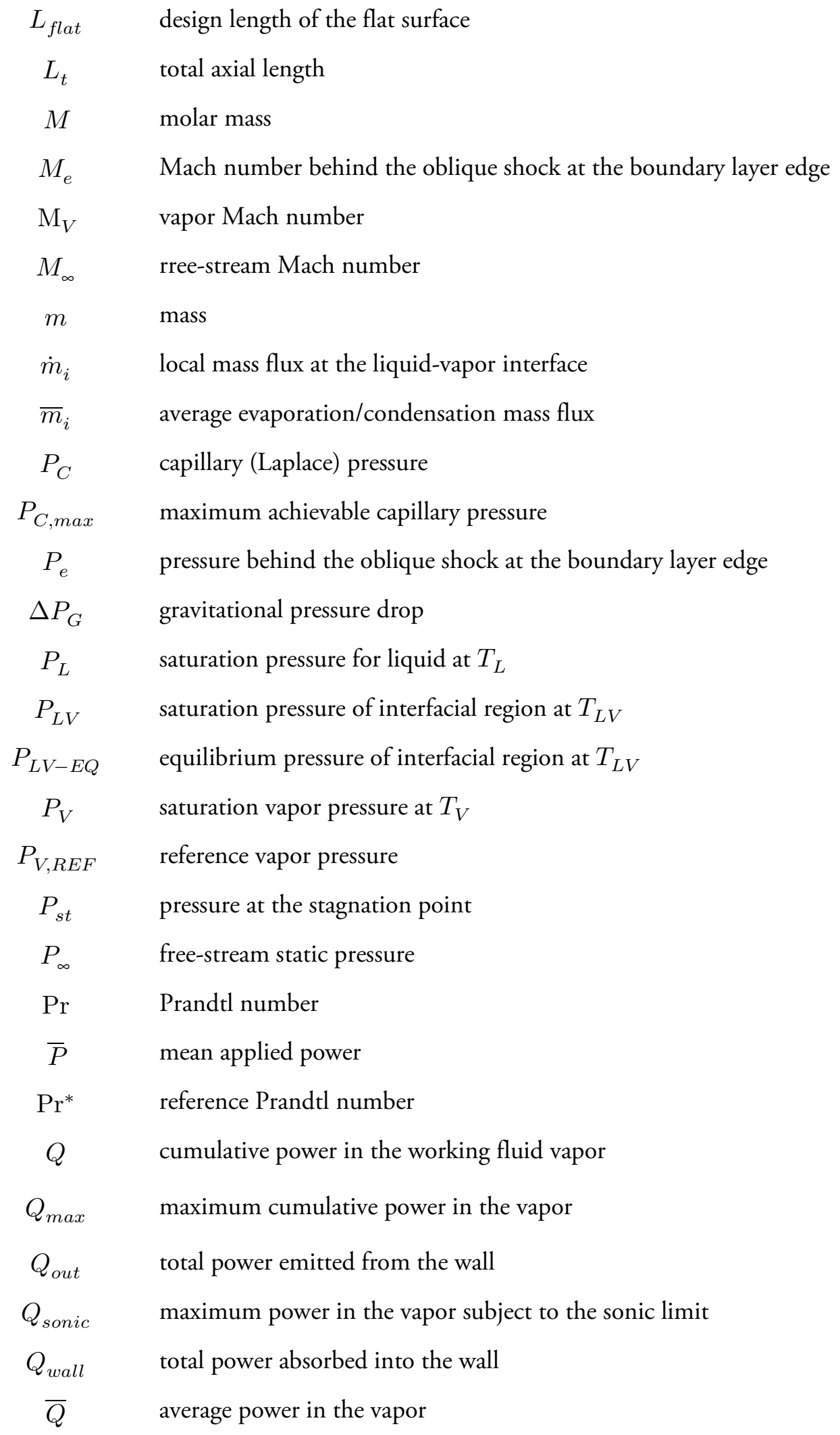




\begin{tabular}{|c|c|}
\hline$q_{b o i l}$ & heat flux at the boiling limit \\
\hline$q_{c a p}$ & heat flux at the capillary limit \\
\hline$q_{f, g w}$ & heat flux to the wall over the flat surface \\
\hline$q_{g w}$ & heat flux from the gas to the wall \\
\hline$q_{K n}$ & heat flux at the Knudsen limit \\
\hline$q_{\max }$ & maximum wall heat flux \\
\hline$q_{r}$ & radiant heat flux \\
\hline$q_{\text {sonic }}$ & heat flux at the sonic limi \\
\hline$q_{s t}$ & stagnation point heat flux \\
\hline$q_{s t, g w}$ & heat flux from the gas to the wall at the stagnation point \\
\hline$q_{t i p, g w}$ & heat flux to the wall around the tip curvature \\
\hline$q_{w}, q_{\text {wall }}$ & heat flux through the wall \\
\hline$q_{\text {yield }}$ & heat flux at the yielding limit \\
\hline$q^{\prime} P h C$ & phase change heat transfer rate per unit length at the liquid-vapor interface \\
\hline$q^{\prime}{ }_{w}$ & heat transfer rate per unit length in the wall \\
\hline$q^{\prime}{ }_{\text {wick }}$ & heat transfer rate per unit length in the wick \\
\hline$R$ & universal gas constant \\
\hline$R_{L E}$ & leading edge radius of curvature \\
\hline$R_{c}$ & circuit resistance \\
\hline$R_{m}$ & meniscus radius \\
\hline$R_{p}$ & pore radius \\
\hline$R_{V}$ & vapor space radius \\
\hline$R_{h}$ & conduction resistance at the heater-wall interface \\
\hline$R_{\text {wall }}$ & radius of the exterior wall surface in the cylindrical heat pipe \\
\hline$R e_{V}$ & working fluid Reynolds number (vapor) \\
\hline$R e_{x}$ & plate Reynolds number \\
\hline$r$ & recovery factor \\
\hline$r_{b}$ & bubble radius \\
\hline$r_{h}$ & hydraulic radius \\
\hline
\end{tabular}




$\begin{array}{cl}s, x, y & \text { coordinate } \\ T_{a w} & \text { adiabatic wall temperature } \\ \Delta T_{b} & \text { critical superheat for bubble formation } \\ T_{e} & \text { temperature behind the oblique shock at the boundary layer edge } \\ T_{h} & \text { heater temperature } \\ T_{i s o} & \text { isothermal operating temperature } \\ T_{s, i} & \text { surface temperature taken by IR } \\ T_{L} & \text { liquid temperature } \\ T_{L V} & \text { liquid-vapor interface temperature } \\ T_{m a x} & \text { maximum temperature of the wall } \\ T_{s} & \text { wall surface temperature } \\ T_{t r} & \text { Knudsen transition temperature } \\ T_{V} & \text { vapor temperature } \\ T_{V, R E F} & \text { reference vapor temperature } \\ T_{w} & \text { wall temperature } \\ T_{\infty} & \text { free-stream temperature, ambient temperature } \\ T^{*} & \text { reference temperature } \\ u_{e} & \text { air velocity behind the oblique shock at the boundary layer edge } \\ u_{s t} & \text { velocity at outer edge of boundary layer at stagnation point } \\ V_{r m s} & \text { root mean square of the voltage } \\ V_{\infty} & \text { free-stream velocity } \\ W & \text { vapor space width } \\ w & \text { meniscus shoulder length } \\ & \end{array}$

\section{Greek}

$\alpha \quad$ thermal expansion coefficient

$\beta \quad$ oblique shock angle, momentum correction coefficient 


\begin{tabular}{|c|c|}
\hline$\gamma$ & ratio of specific heat capacities \\
\hline$\varepsilon$ & wick porosity \\
\hline$\epsilon$ & emissivity \\
\hline$\theta$ & angle between flight direction and leading edge radius vector \\
\hline$\theta_{o}$ & tip curvature angle \\
\hline$\kappa$ & thin film length (active in evaporation), Boltzmann constant \\
\hline$\lambda$ & latent heat of vaporization \\
\hline$\lambda_{m}$ & mean free path \\
\hline$\mu_{e}$ & viscosity behind the oblique shock at the boundary layer edge \\
\hline$\mu_{V}$ & working fluid vapor viscosity \\
\hline$\mu_{s t}$ & air viscosity at the stagnation point \\
\hline$\nu$ & Poisson's ratio \\
\hline$\rho$ & material density \\
\hline$\rho_{L}$ & working fluid liquid density \\
\hline$\rho_{V}$ & working fluid vapor density \\
\hline$\rho_{V, o}$ & vapor stagnation density \\
\hline$\rho_{e}$ & density behind the oblique shock at the boundary layer edge \\
\hline$\rho_{\text {wick }}$ & wick density \\
\hline$\rho_{\text {solid }}$ & density of solid wick material \\
\hline$\rho_{s t}$ & air density at the stagnation point \\
\hline$\rho_{\infty}$ & free-steam density \\
\hline$\sigma$ & Stefan-Boltzmann constant \\
\hline$\sigma_{L V}$ & liquid surface tension \\
\hline$\sigma_{d}$ & molecule diameter \\
\hline$\sigma_{f}$ & fracture strength \\
\hline$\sigma_{t h}$ & thermo-elastic stress \\
\hline$\sigma_{y}$ & yield strength \\
\hline
\end{tabular}


$\varphi \quad$ wedge half angle

$\omega \quad$ contact angle 


\section{Chapter 1. Introduction}

\subsection{Rationale for dissertation}

Ever since the Wright brothers' famous 1903 first flight in Kill Devil Hills, North Carolina, mankind has aspired for faster flight vehicles which push the limits of engineering. Beyond the natural human desire to "go faster", innovation has been driven by the need for vehicles which re-enter the Earth's atmosphere (or other planetary atmospheres) at speeds approaching Mach 25, efforts to transport people and goods on transatlantic flights in under an hour, and facilitate critical military aircraft and missile systems.

The severe thermal environment to which these hypersonic vehicles are subjected during flight pose significant technical challenges. The hypersonic regime, generally considered to be Mach 5 or higher, is defined as "the realm of speed wherein the physics of flows is 
dominated by aerodynamic heating" [1]. Exposure to extremely high temperatures (and heat fluxes) during hypersonic flight through the atmosphere is the result of two phenomena [2]:

(a) Air stagnation: as the air molecules ahead of a hypersonic structure are decelerated across the bow shock, the kinetic energy is converted to internal energy of the gas, generating very high temperatures at leading edges.

(b) Skin friction: downstream of the leading edge shock, a boundary layer forms against the vehicle surface and extreme viscous dissipation creates high temperatures in the gas flow.

In both situations, the thermophysical (and, at high enough temperatures, thermochemical) properties of air play a central role in the gas temperature in contact with the vehicle surface. At sea level, the density of air is $1.225 \mathrm{~kg} / \mathrm{m}^{3}$ (about $1 / 1000$ that of water) and decreases with altitude as Earth's atmosphere (continuum regime) gives way to the free molecular (rarified) regime of space as shown in Figure 1.1. The U.S. Space Shuttle, reenters Earth's atmosphere (at an altitude of $120 \mathrm{~km}$ ) at a speed of about $8 \mathrm{~km} / \mathrm{s}$ or approximately 28 times the speed of sound. In this uppermost region of the atmosphere, the temperature behind the bow shock reaches $7,000 \mathrm{~K}[3]$. For a lunar orbiting vehicle returning to Earth, vehicle speeds approach $11 \mathrm{~km} / \mathrm{s}$ and the bow shock can reach $11,600 \mathrm{~K}$ (almost twice that of the Sun's surface [2]). 


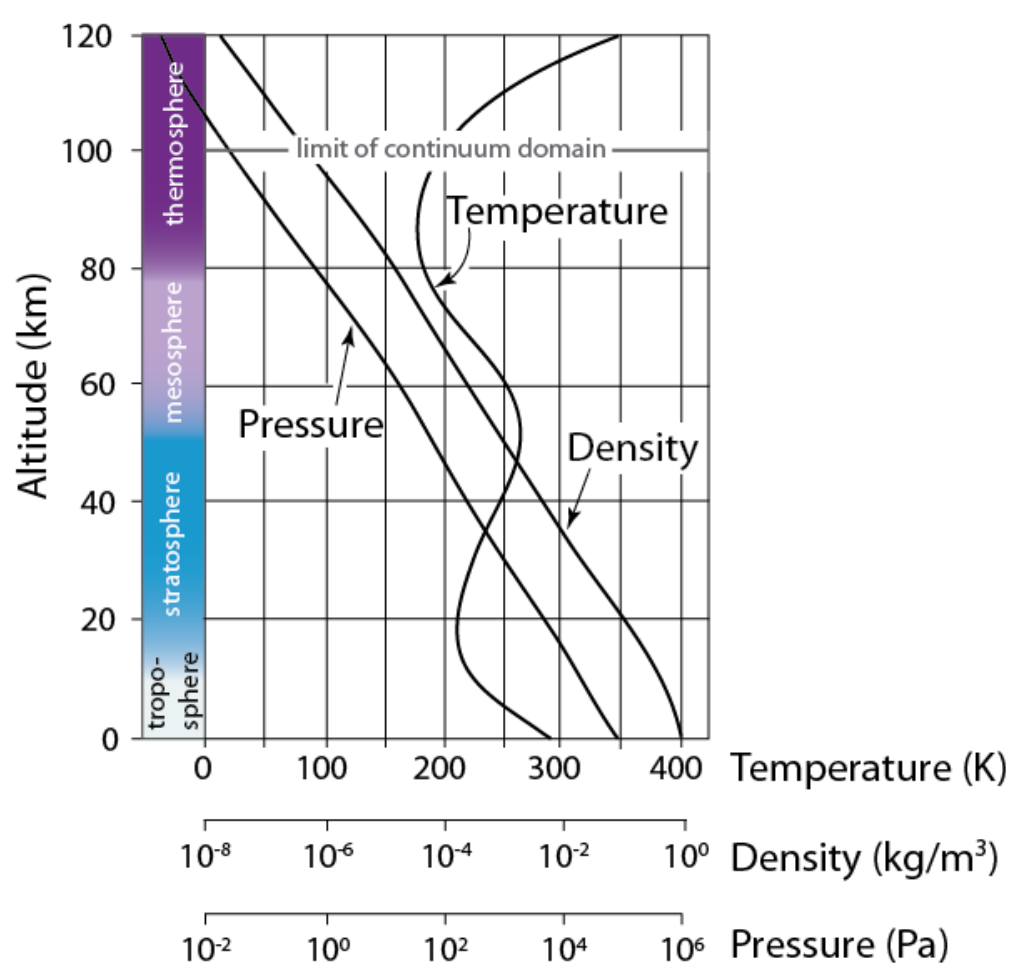

Figure 1.1. Thermodynamic properties of air as a function of altitude based on the U.S. Standard Atmosphere [4].

At lower altitudes, where the ambient pressure and density are much higher, stagnation and viscous temperature effects are exacerbated. For example, while a Mach 6 vehicle will generate a temperature behind the bow shock of $1,300 \mathrm{~K}$ at an altitude of $100 \mathrm{~km}$, it is almost twice that, $2,300 \mathrm{~K}$, at $10 \mathrm{~km}$.

As a result, some of the most challenging thermal problems in aerospace vehicle design are encountered at the leading edges of hypersonic airbreathing vehicles. The scramjet engine, which has been used in vehicle designs to attain Mach 10 [5], necessitate low-altitude, atmospheric flight for their efficient operation. The propulsion system 
combusts a mixture of hydrocarbon fuel and compressed atmospheric air which contains about $20 \%$ oxygen. The advantage of this system is that the oxidizing reactant used for fuel combustion is taken from the atmosphere through which it flies, lightening on-board fuel requirements [5].

Figure 1.2 shows a schematic of HyFly, a hypersonic vehicle proposed by the Defense Advanced Research Program Agency (DARPA) and Office of Naval Research (ONR). The goal of the demonstrator program was to investigate low altitude Mach 6 cruise for over 600 nautical miles using a scramjet engine [6]. NASA has also tested Mach 10 air breathing vehicle concepts in the $\mathrm{X}-43 \mathrm{~A}$ program[7]. While the entire vehicle body is subjected to atmospheric heating due to drag, the leading edges - including the nose region, tail edges, and scramjet inlets - are exposed to intense, highly localized stagnation point heating and a severe thermal environment. 


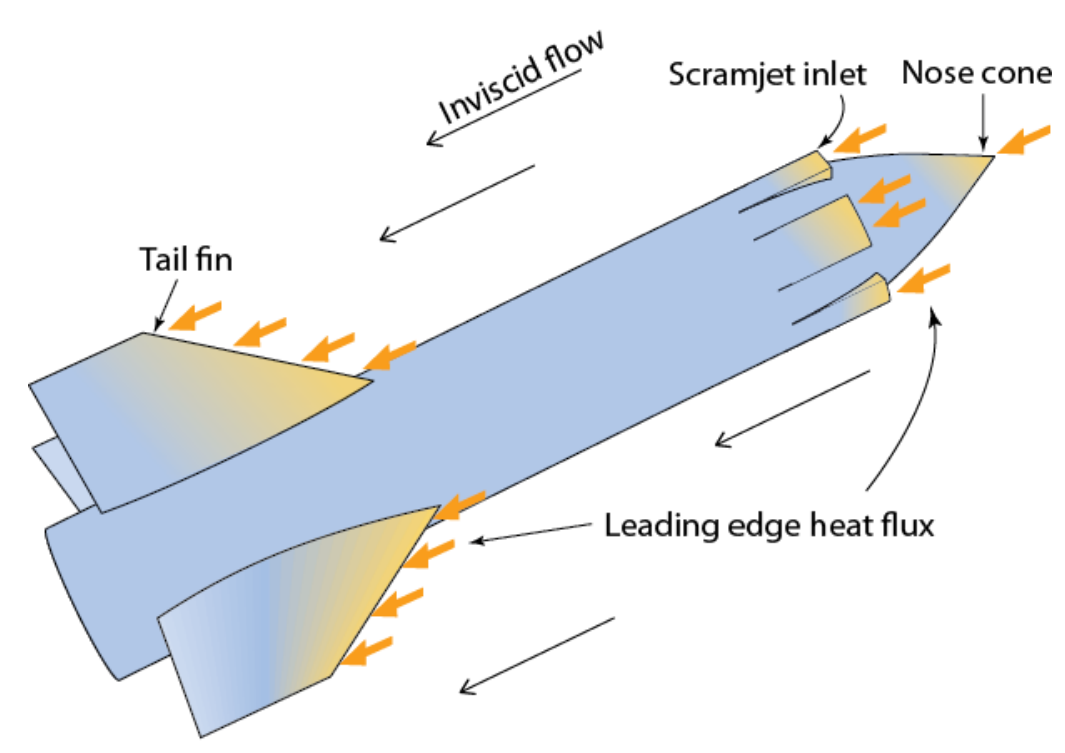

Figure 1.2. Schematic illustration of the DARPA/ONR hypersonic HyFly vehicle showing areas of stagnation point heating at the nose cone, scramjet inlet, and tail fin leading edges.

As a rule of thumb, efficient combustion in air-breathing scramjet engines requires a dynamic pressure of $\rho_{\infty} V_{\infty}^{2} / 2=48 \mathrm{kPa}$, where $\rho_{\infty}$ is the free-stream (atmospheric) air density and $V_{\infty}$ the vehicle velocity[8]. This criterion is shown in Figure 1.3(a), which shows that flight at higher altitudes (lower air density) requires an increasingly higher Mach number to maintain the necessary dynamic pressure. The scramjet flight trajectory is therefore dictated by any two of these three variables; the third variable becomes fixed. This is important to note upfront because these variables play a role in stagnation point heating.

Unlike reentry vehicles such as the Space Shuttle Orbiter or its replacement, the Orion Multipurpose Crew Vehicle (under development at NASA), a low drag aerodynamic 
design is paramount for scramjet vehicles. Figure 1.3(b) shows the influence of the leading edge radius on the stagnation heat flux for Mach 6, 7, and 8 flight at a constant dynamic pressure of $48 \mathrm{kPa}$. The plotted relationship has the heat flux inversely proportional to the square root of the leading edge radius. Reentry vehicle design is primarily concerned with slowing the vehicle as it reenters Earth's atmosphere, so blunt leading edges are used not only for deceleration, but also to mitigate the intense heating (e.g. the Shuttle uses a radius of $600 \mathrm{~mm}[9])$. In contrast, scramjets must be able to accelerate through the atmosphere and therefore make use of sharp leading edges to minimize drag. For example, the HyFly program scramjet inlet design proposed a $3 \mathrm{~mm}$ leading edge radius[10]. Such sharp tips create exceptional thermal challenges (discussed in Chapter 2) that require innovative solutions. 

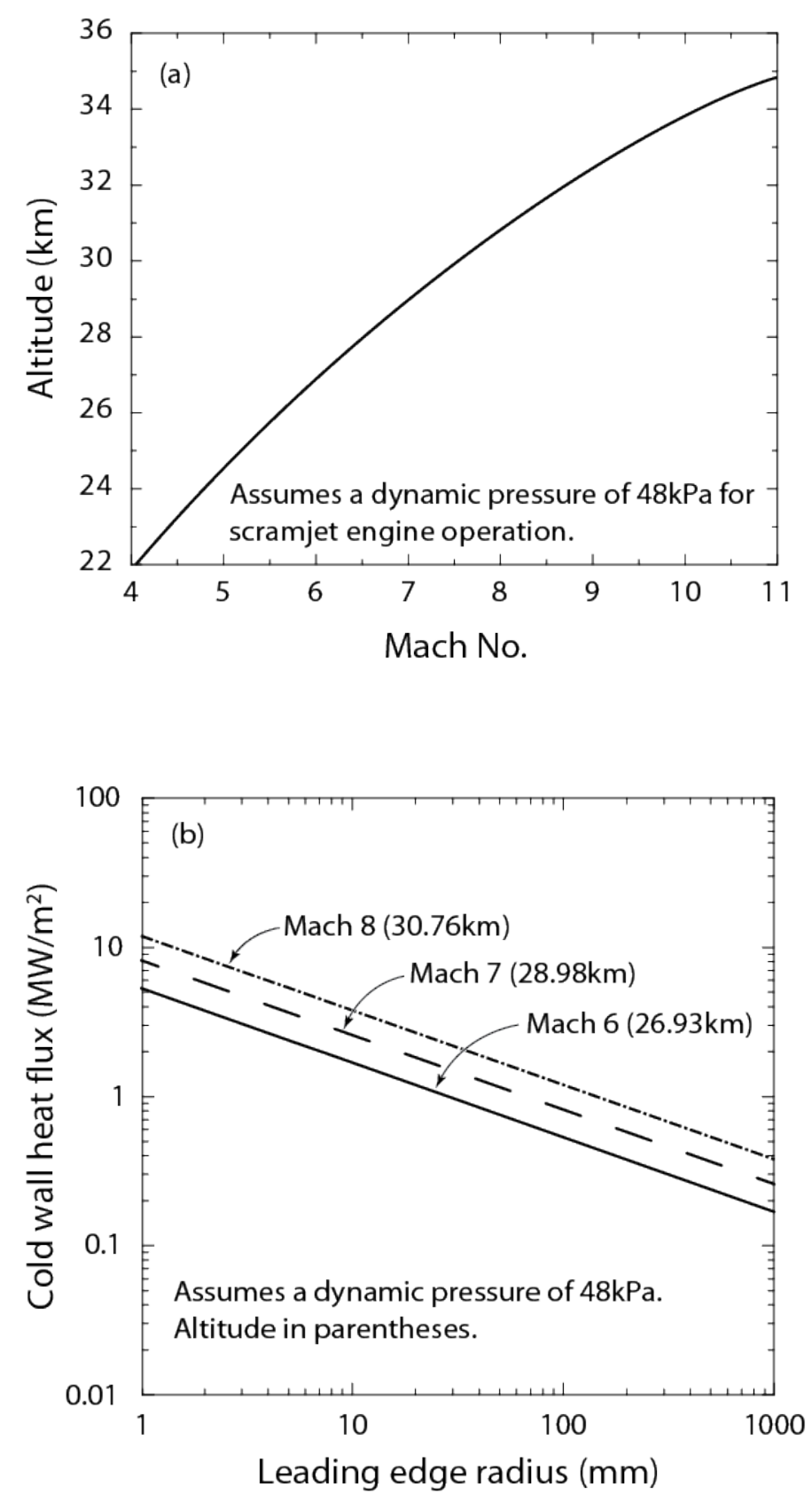

Figure 1.3. (a) The relationship between altitude and Mach number for a dynamic pressure of $40 \mathrm{kPa}$. (b) The influence of leading edge radius on the stagnation point heat flux assuming a cold wall (the temperature of the gas >> temperature of the vehicle). 
Materials are an enabling technology in high speed air vehicle development because the severe thermal environment at a vehicle's leading edges requires the use of a thermal protection system (TPS) to maintain the underlying airframe structure within allowable material temperature limits. Example TPS's used for managing the intense heat at leading edges include; ( $i$ ) the ablative coatings of NASA's Mercury, Gemini, and Apollo vehicles [1] and (ii) the reusable reinforced carbon-carbon (RCC) system of the Space Shuttle Orbiter [11]. The ablative approach absorbs heat by endothermic transformation (phase and/or chemical change to the polymeric coating). The heat is dissipated from the vehicle as the single-use coating eventually vaporizes. The RCC approach manages the intense heat by operating at high-temperatures and radiating heat to its surroundings. The effectiveness of both systems is predicated on keeping the heat flux that impinges upon the susceptible airframe below a critical level.

This dissertation explores an alternative TPS concept which seeks to redistribute the heat from the leading edge and thereby eliminate leading edge hot spots. It makes use of high thermal conductance heat pipes coupled to the leading edge so that the thermal load may be redistributed from a high heat flux location (at the stagnation point) to regions where it can be effectively radiated from the vehicle. The sealed system concept is based upon the evaporation of a fluid near the heat source that sets up a region of elevated vapor pressure inside the pipe. The latent heat is transported down the resulting pressure gradient by the 
vapor stream where it condenses at cooler regions, releasing the heat for removal. Replenishment of the condensed working fluid to the evaporator region is accomplished through the capillary pumping action of a porous wick which lines the interior surface of the pipe. The result might be a nearly isothermal leading edge that would reduce the maximum temperature and thermal stress.

\subsection{Dissertation objectives}

The objectives of this dissertation are to: (i) explore the potential of heat pipe-based metallic leading edges for hypersonic vehicles; (ii) establish a methodology for their design; (iii) develop methods for fabricating high-temperature leading edges from appropriate high temperature materials; (iv) investigate their performance under simulated hypersonic heat fluxes; and $(v)$ develop models that link heat transfer limits to materials and structure.

\subsection{Dissertation outline}

The dissertation is organized as follows: Chapter 2 provides a background on the thermomechanical issues located at the leading edges of airbreathing hypersonic vehicles, an overview of TPS solutions, and an introduction to the metallic thermal spreading concept proposed here. Chapter 3 reviews the relevant theory for calculating the heat flux at the leading edge and proposes a design methodology for its design. Chapter 4 examines the 
shortcomings of a popular heat transfer limit model and an improved model is developed. The heat transfer limits of the leading edge heat spreader are explored in Chapter 5, including a new limit which has not received attention previously. Chapter 6 describes the fabrication and testing of a low temperature system whose results are used to validate the design methodology. Chapter 7 details the design, fabrication, and testing of a high temperature leading edge heat spreader. A discussion on three proposed high temperature case material and fluid combinations using the models is presented in Chapter 8. The dissertation concludes with a summary of the main findings of this work in Chapter 9. 


\section{Chapter 2. Thermal protection concepts}

Despite significant advances in high temperature materials, air vehicle design, aerothermodynamic /material modeling efforts over the last 50 years, the TPS failure on the U.S. Space Shuttle Columbia in February 2003 exposed a need for more robust leading edge materials and structures for the extreme hypersonic environment. Growing interest in low altitude hypersonic flight and the success of NASA's X-43A Mach 10 airbreathing vehicle [7] is further increasing interest in novel approaches to thermal protection at leading edges. This chapter presents some of the factors that must be addressed in the design of a TPS. First, the thermal requirements unique to sharp leading edges are presented ${ }^{1}$ and followed with an overview of current TPS concepts. Three of these concepts are reviewed in more detail together with an assessment of their viability for scramjet leading edges. The rationale for a

\footnotetext{
1 A detailed analysis is presented in Chapter 3.
} 
metallic leading edge heat spreading concept is then presented, and its potential for overcoming the deficiencies of other systems is identified.

\subsection{The hypersonic environment}

\section{1(a) Elevated temperatures}

The impact of atmospheric molecules with a hypersonic structure generates high temperatures in regions of flow stagnation. The National AeroSpace Plane (NASP), an airbreathing hypersonic vehicle concept whose program was eventually cancelled in 1993, was intended for Mach 8 flight with leading edge gas temperatures approaching $1,650^{\circ} \mathrm{C}$ [12]. As speeds increase, future vehicles will create stagnation regions near the leading edges where temperatures approach 2,000 to $2,400^{\circ} \mathrm{C}$ [13]. Figure 2.1 is a material property chart showing maximum service temperature and specific strength (yield or fracture strength normalized by its density). Low cost materials suited for aerospace have a high specific strength, such as aluminum, titanium, and lightweight composites. Aluminum and titanium alloys have maximum service temperatures of approximately $200^{\circ} \mathrm{C}$ and $500^{\circ} \mathrm{C}$, respectively. Nickel alloys push this thermal envelope to $1,000^{\circ} \mathrm{C}$. Their specific strength is lower than titanium so their use must be selective and likely unacceptable for the entire airframe or skin. If the heat in the stagnation region were to equilibrate with the leading edges structure, the material temperatures would far exceed the thermo-structural limit of conventional polymer 
and metallic matrix composite airframe materials. Ceramic composites, such as a silicon carbide fiber in a silicon carbide matrix $(\mathrm{SiC} / \mathrm{SiC})$ and a carbon fiber in a carbon matrix $(\mathrm{C} / \mathrm{C})$, have temperature service limited by their reactive chemistry with the atmosphere and require environmental barrier coatings which have proven difficult to develop. It is evident that either new materials are needed, or a TPS is required to protect the airframe from stagnated region temperatures, or both.

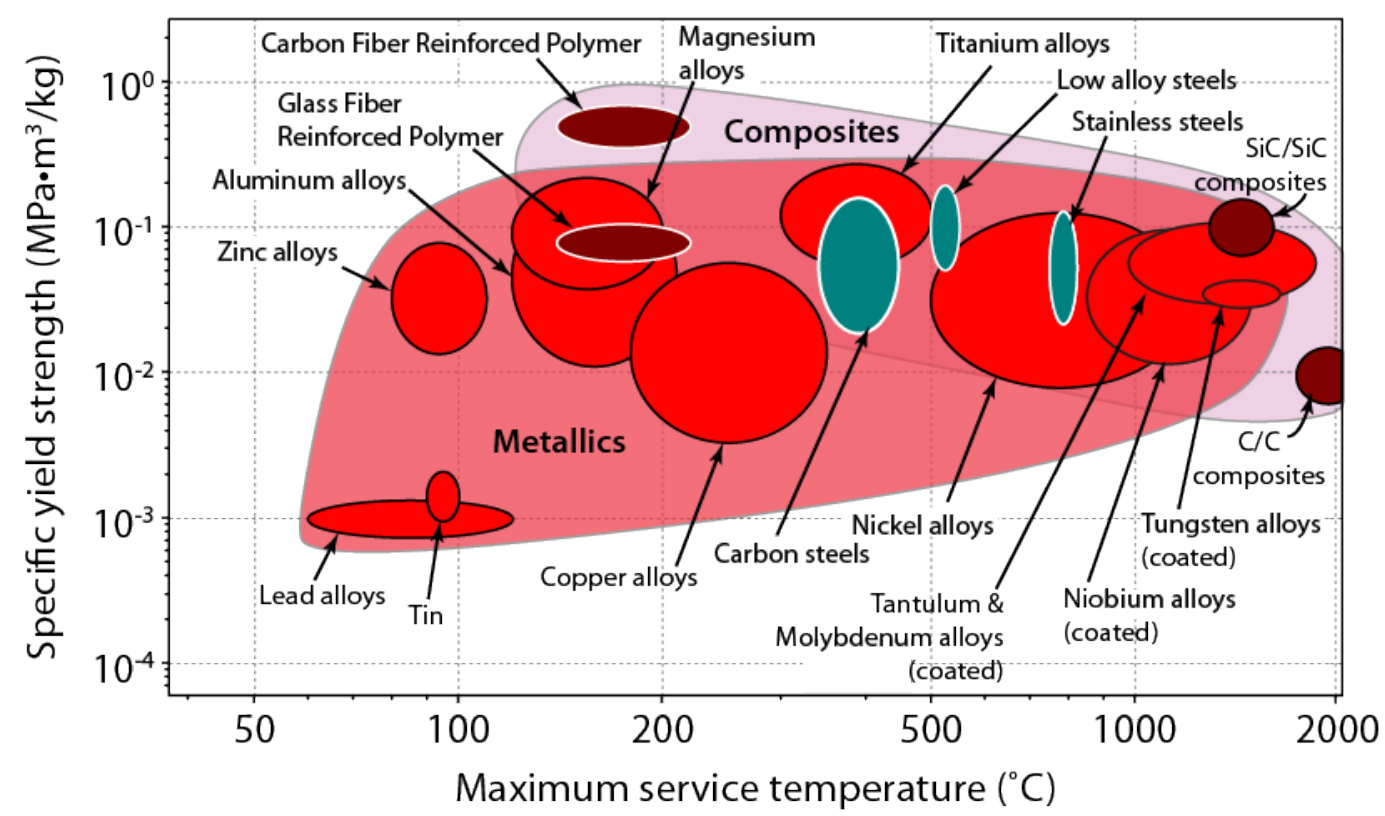

Figure 2.1. Material property chart of yield strength normalized by density and maximum service temperature for common metals and aerospace composites. 


\section{1(b) Thermal gradients and stresses}

Temperature alone does not fully characterize the aerothermal environment at the leading edge. A first order approximation ${ }^{2}$ of the heat flux incident upon a cold surface at the stagnation point, $q_{s t}\left(\mathrm{~W} / \mathrm{cm}^{2}\right)$, has been proposed by Anderson [3];

$$
q_{s t}=1.83 \times 10^{-8}\left(\frac{\rho_{\infty}}{R_{L E}}\right)^{1 / 2} V_{\infty}^{3}
$$

where $\rho_{\infty}\left(\mathrm{kg} / \mathrm{m}^{3}\right)$ and $V_{\infty}(\mathrm{m} / \mathrm{s})$ are the free stream density and velocity, respectively, and $R_{L E}(\mathrm{~m})$ is the leading edge radius. The flux is a very strong function of the vehicle speed. It also increases with reduction in altitude and leading edge radius.

The heat flux for laminar flow on the flat surface aft of the leading edge tip, $q_{f}$, can be analyzed by flat plate flow theory, which gives an estimated [3];

$$
q_{f}=2.53 \times 10^{-9} \sin \varphi\left(\frac{\rho_{\infty} \cos \varphi}{\eta}\right)^{1 / 2} V_{\infty}^{3.2}
$$

where $\varphi$ is the angle of the flat surface to the flow (for the case of level flight, this is the wedge half angle) and $\eta(\mathrm{m})$ is the distance measured along the body surface from a virtual point extended past the curved tip to the horizontal. Both of these geometric parameters are defined in Figure 2.2(a). The free stream properties use the same units above.

2 The physical principles and mathematical theory behind a more accurate heat flux estimation is presented in Chapter 3. 
Equation (2.2) shows that the flux over the flat portion falls away from the leading edge tip and is strongly dependent on the vehicle velocity. The flux decreases with increasing altitude and decreasing angle $\varphi$. A plot of these equations is shown in Figure 2.2 for the heat flux distribution around a vehicle's leading edge traveling Mach 7 at an altitude of $29 \mathrm{~km}$ above sea level $\left(\rho_{\infty}=2.16 \times 10^{-2} \mathrm{~kg} / \mathrm{m}^{3}\right.$ and $V_{\infty}=2.1 \mathrm{~km} / \mathrm{s}$. The leading edge tip radius is taken as $3 \mathrm{~mm}$ and the wedge half angle is $6^{\circ}$. The peak heat flux of $460 \mathrm{~W} / \mathrm{cm}^{2}$ occurs at the tip and rapidly decreases around the curved radius toward the flat surface. The heat flux behind the leading edge falls to $6 \mathrm{~W} / \mathrm{cm}^{2}$ at $x / L=1$, a nearly hundred-fold decrease from that at the tip. Such a rapid drop in heat flux would result in a significant thermal gradient. The thermal expansion of a material is proportional to its temperature rise; therefore, a variation in temperature, $\Delta T$, within a material induces a thermal stress, $\sigma_{t h}$, given by [14];

$$
\sigma_{t h}=\frac{E \alpha}{1-v} \Delta T
$$

where, $\alpha$ is the material's thermal expansion coefficient, $E$ is the material's Young's modulus, $\Delta T$ is the temperature difference within the material, and $v$ is the material's Poisson's ratio.

Equation (2.3) shows that the thermal stresses are proportional to the temperature difference and Young's modulus (which usually increases with material melting temperature, making high temperature materials susceptible to large thermal stresses). The thermal stresses can be avoided by using low stiffness materials, which conflicts with aerodynamic design requirements, or by the use of very low thermal expansion materials. 
An alternative strategy is to use a high thermal conductivity material which would reduce $\Delta T$ since, by way of Fourier's law [15];

$$
\Delta T \propto \frac{1}{k}
$$

where $k$ is the material's thermal conductivity. Substituting into equation (2.3) gives;

$$
\sigma_{t h} \propto \frac{E \alpha}{(1-v) k}
$$

To avoid yielding or brittle fracture, the materials strength must exceed the induced stresses, or $\sigma_{y}>\sigma_{t h}$ where $\sigma_{y}$ is the material's yield or fracture strength. At the limit of failure;

$$
\sigma_{t h}=\sigma_{y} \propto \frac{E \alpha}{(1-v) k}
$$




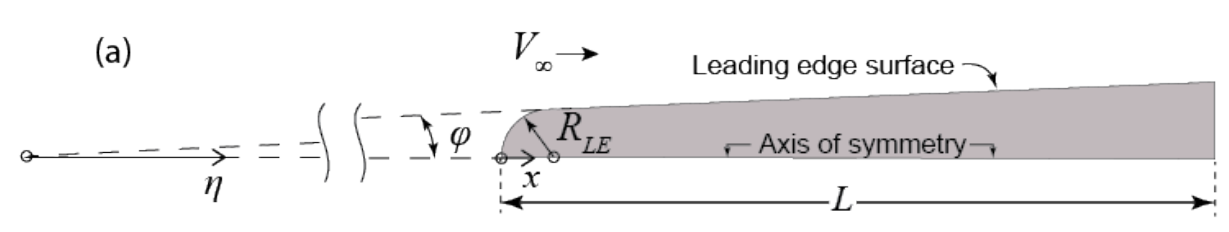

(b)

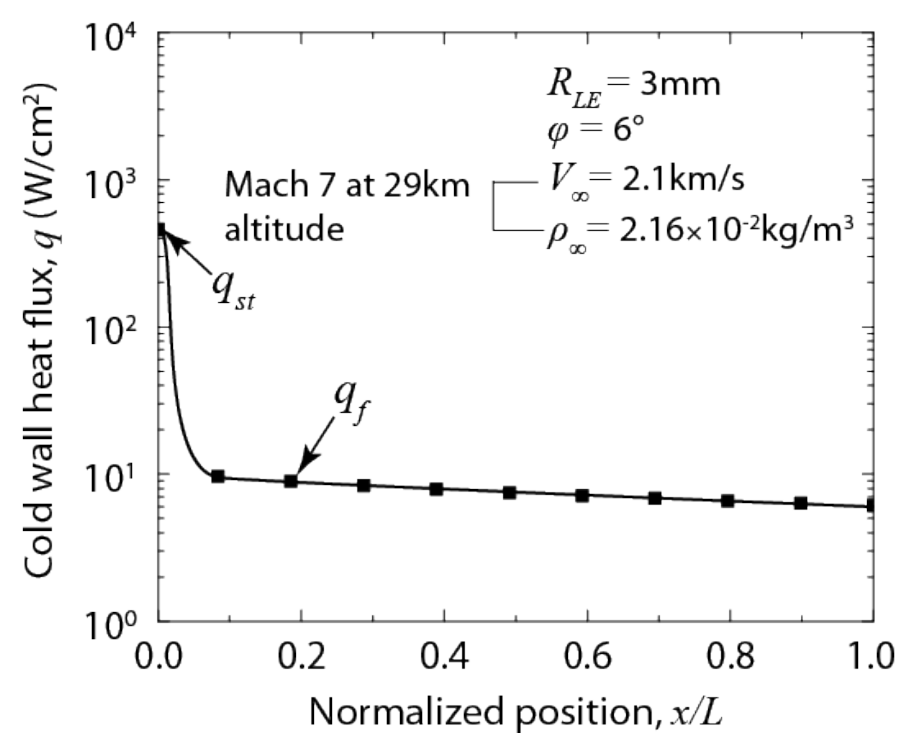

Figure 2.2. (a) Leading edge geometry defined by tip radius $R_{L E}$ and half angle $\varphi$, and (b) the heat flux profile along $x$ for a wedge with $R_{L E}=3 \mathrm{~mm}$ and $\varphi=6^{\circ}$ at Mach 7 (29.0km).

Equation (2.6) shows that a combination of material properties governs thermomechanical failure: the use of a material of high thermal conductivity, low Poisson's ratio, low stiffness, and low thermal expansion coefficient reduces the risk of failure, especially if high strength materials are used. Figure 2.3 is a material property chart where this combination of material properties is the abscissa and the material strength is plotted on the ordinate axis. Only materials with a maximum service temperature above $800^{\circ} \mathrm{C}$ are 
shown $^{3}$. Materials with the most desirable properties are in the upper left quadrant while those with the least are in the lower right quadrant. The refractory metallics exhibit high strength but their relatively large thermal expansion coefficient can lead to large stresses. There is a material gap between the refractory metallics and diamond which, if filled, has potential as an ideal TPS material.

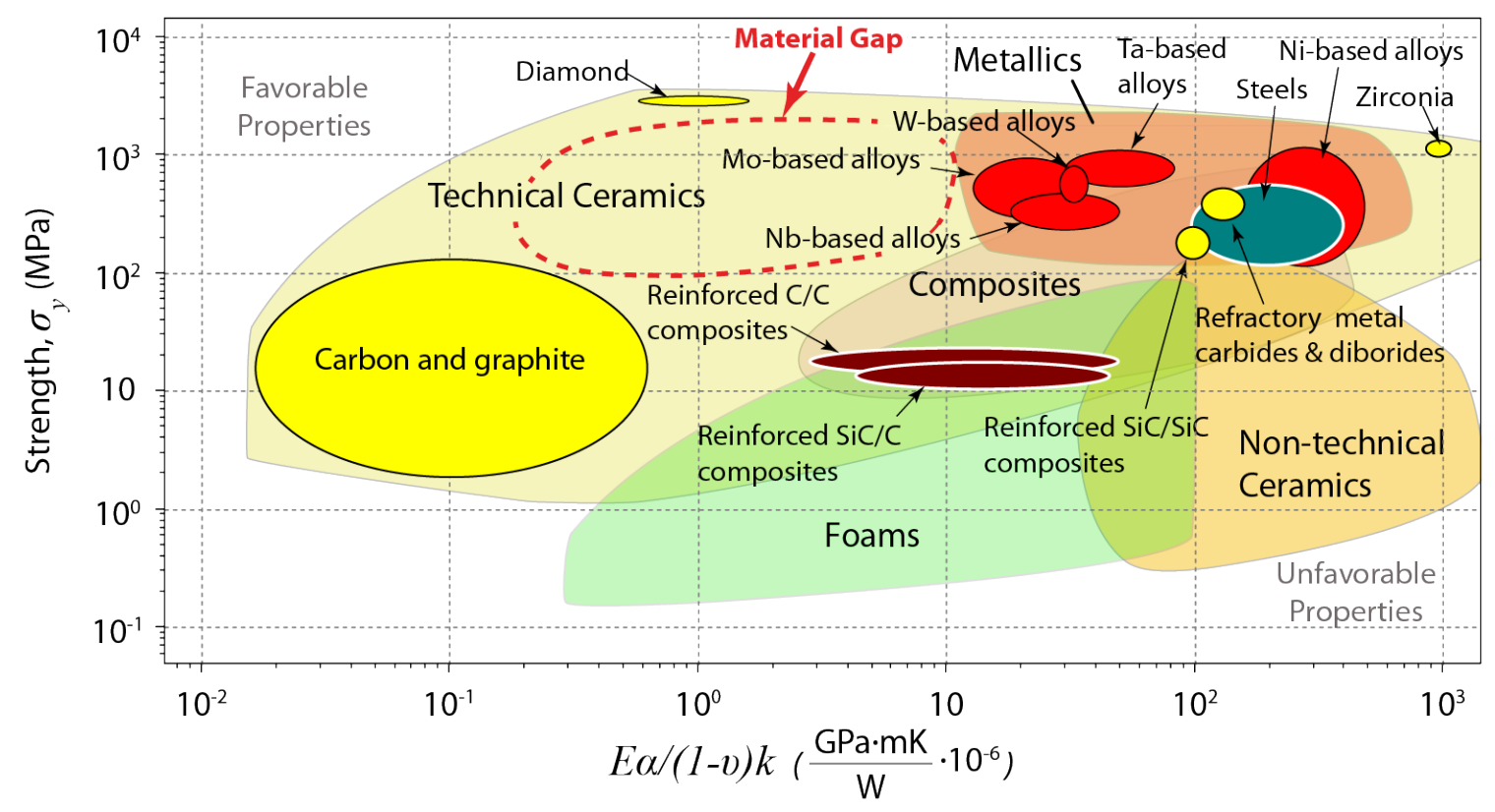

Figure 2.3. Chart showing the relative potential of materials for localized high heat flux, high temperature environments. The most favorable materials are ones with high yield strength, high thermal conductivity, low stiffness, and low thermal expansion, which can be found in the upper left quadrant. There is a material gap between diamond and the refractory metallics.

3 Refractory metallics are assumed to have adherent environmental barrier coatings for protection from oxidation above $800^{\circ} \mathrm{C}$ for the purpose of the figure. 


\section{1(c) Chemically reacting flows}

In the region of flow stagnation ahead of the leading edge, the kinetic energy of the free stream is reduced to zero and converted to internal energy (heat) of the gas which, at high enough temperatures, becomes chemically reactive. The vibrational energy of the molecules becomes significant at $530^{\circ} \mathrm{C}$ and standard pressure $(101 \mathrm{kPa})$ and at $2,200^{\circ} \mathrm{C}, \mathrm{O}_{2}$ starts to dissociate and become highly reactive. At $3,700^{\circ} \mathrm{C} \mathrm{N}_{2}$ dissociation also begins, further increasing the reactivity of atmospheric gas. Monotonic $\mathrm{O}$ and $\mathrm{N}$ start to ionize above $8,730^{\circ} \mathrm{C}$, creating highly reactive ionic gases that can degrade the vehicle surface [2], [3], [16]. For a reusable TPS, these gas-surface interactions become an increasing concern for some materials that might be considered for a TPS [17].

To further investigate this issue, an altitude-vehicle velocity map is shown in Figure 2.4. Regions of vibrational excitation, dissociation, and ionization have been superimposed on the map together with flight velocity and altitude combinations associated with various hypersonic vehicles. As the atmospheric pressure decreases (with increasing altitude), the dissociation and ionization temperatures decrease, which is captured by the leftward leaning orientation of each region. Reentry vehicles enter the atmosphere at high velocities (e.g. the U.S. Space Shuttle at nearly Mach $28(8 \mathrm{~km} / \mathrm{s}))$ and gas enthalpies are well within the chemically reactive nitrogen and oxygen dissociation regions [18]. Reactions between the gas and the surface can be exothermic, further increasing the temperatures (and heat flux) in the boundary layer [19]. Because of the requirement for oxygen combustion (which requires 
lower altitudes for higher oxygen densities), most scramjet powered vehicle concepts (X-15,

$\mathrm{X} 51 \mathrm{~A}, \mathrm{HiFire})$ operate in a regime in which they are not subjected to a chemically reacting gas.

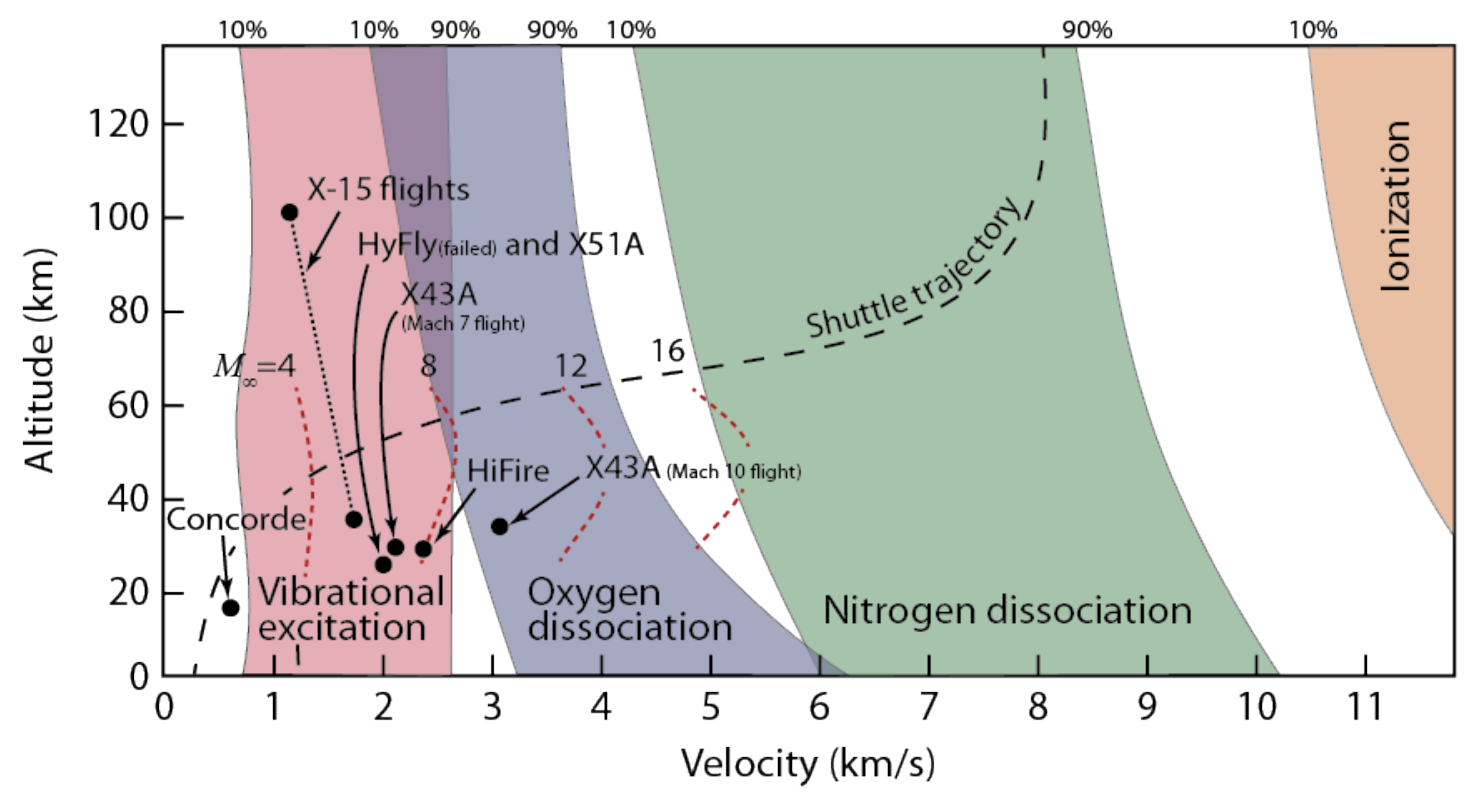

Figure 2.4. Velocity-altitude map showing the flight condition at which vibrational excitation and chemical reactivity effects of the gas are significant. Most airbreathing flight trajectories are subjected to significant vibrational excitation effects only. Altitude-velocity map from [3]. IsoMach lines (....) have been added for reference. Flight data compiled from [3], [7], [20], [21], [22].

\section{1(d) Secondary issues}

The combined aerodynamic and thermal loads present additional challenges which must be addressed during material selection[23]. A reusable TPS intended to have a multiple flight lifespan must resist high temperature creep. Acoustic loads on airbreathing vehicles can reach as high as $180 \mathrm{~dB}$; fatigue generated by boundary layer turbulence and engine vibrations operating at natural frequencies of structures must be considered [24]. Surface 
roughness can "trip" laminar boundary layer flow on the vehicle skin, generating an increase in heat flux from turbulent flow. While many factors influence the tripping point, including roughness height, boundary-layer thickness, and Reynolds number, surface elements down to several thousandths of an inch can be problematic for hypersonic vehicle skins [25].

\subsection{The TPS concept}

In order to be viable, a scramjet's leading edge TPS must be manufacturable to a sharp radius, be made from a material capable of operating in an oxidizing atmosphere, and should minimize parasitic mass. Figure 2.5 depicts some of the many TPS concepts proposed for high speed flight. They can be categorized as either passive or active. A passive TPS requires no continuous supply of coolant for the removal of heat. In contrast, forced (pumped) convective flow of a liquid coolant is used for the absorption and removal of heat in a TPS of the active type. 

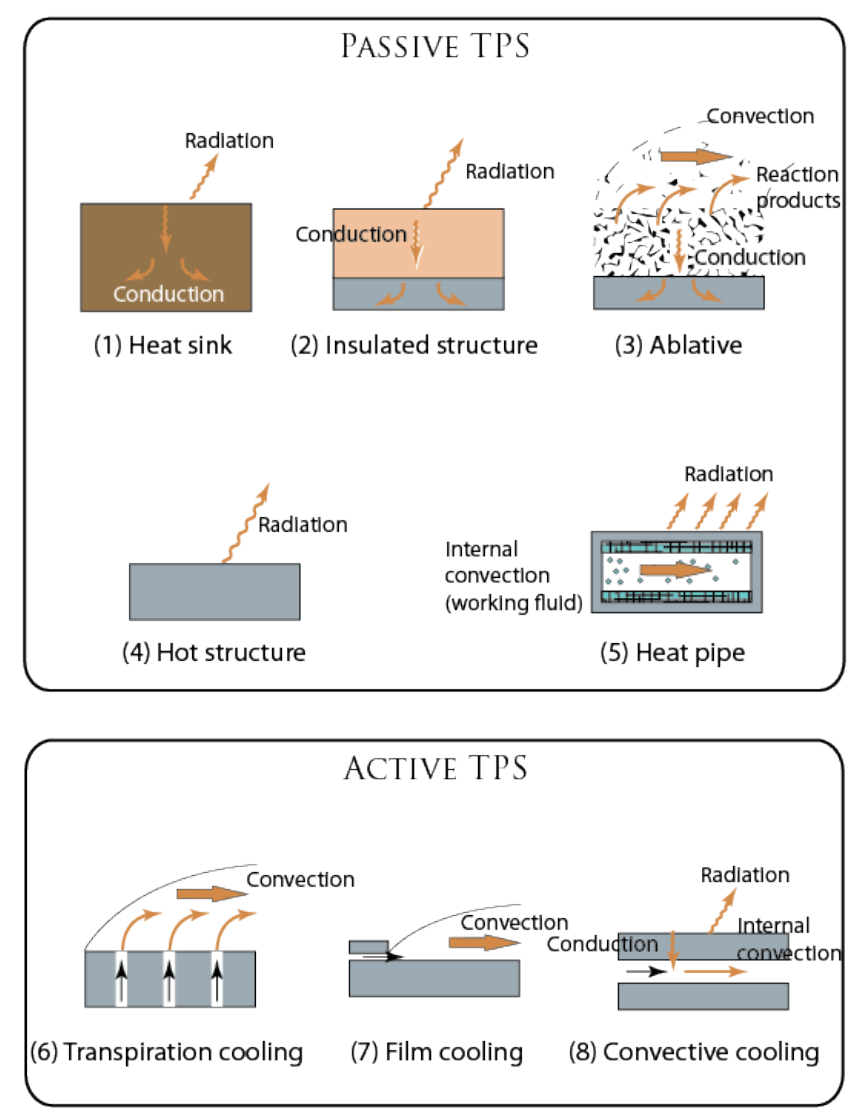

Figure 2.5. Various TPS concepts in operation or proposed for use on hypersonic vehicles. Adapted and modified from [26].

Each concept relies on widely differing operating principles:

(1) Heat sink concepts. Heat is absorbed and stored in a structural material having a combination of high thermal conductivity $(k)$ for uniform heat spreading throughout the sink material and high heat capacity (given by the volumetric heat capacity, i.e. the product of specific heat capacity, $c_{p}$, and density, $\rho$ ) for thermal storage. This is quantified by a material's thermal effusivity, $e$, which is a measure of its ability to absorb heat [15]; 


$$
e=\sqrt{k \rho c_{p}}
$$

The first heat sink concepts utilized copper $\left(e=3.19 \mathrm{Ws}^{1 / 2} \mathrm{~cm}^{-2} \mathrm{~K}^{-1}\right)$ and were used for the nose of an InterContinental Ballistic Missile in the 1950s [27]. Beryllium heat sinks ( $e=2.61 \mathrm{Ws}^{1 / 2} \mathrm{~cm}^{-2} \mathrm{~K}^{-1}$ ) were at one time considered for the reentry capsule of the first manned space flights of the Mercury program [27], [28]. In order to be self insulating, the high thermal conductance heat sink materials must be of sufficient mass to store the total heat accumulated during flight [29]. This conflicts with the design objective of minimum mass for an airbreathing propulsion system. Flight times with long durations of heating would require more massive heat sinks. While not an airbreathing vehicle (which imposes even stricter mass requirements on vehicles), NASA thermal engineers concluded the required mass for a heat sink TPS in the Mercury program was prohibitive [1].

(2) Insulating TPS. An insulating TPS either incorporates a high temperature, low thermal conductance ceramic insulation (often in the form of widely spaced woven or matted fibers or a closed cell foam) to resist thermal diffusion to a susceptible aerostructure or make use of a stand-off design which introduces an insulating gas -filled or vacuum gap between the hot skin and airframe. This approach has been limited to large acreage, non-structural systems since the combination of low thermal conductivity and low fracture toughness of highly porous materials make them unsuitable for use on leading edges that must sustain 
large thermo-mechanical stress. With the exception of the nose cone and leading edges, the Space Shuttle's skin is protected from the hot gases of reentry with an insulating TPS [30].

(3) Ablative. An ablative TPS makes use of a single-use coating that accommodates the heat by way of a combination of endothermic phase changes, chemical changes, and/or (evaporative) mass loss [31].

(4) Hot structure. Structural components of the airframe can be designed to withstand high temperatures by using a high emissivity surface or coating to reradiate the heat. At steady state, hot TPS structures are designed to operate near their adiabatic temperature [12], [32], which is the temperature at which heating of the vehicle's surface balances the radiated thermal energy from the vehicle (i.e. no net energy absorption through the vehicle skin). Reinforced carbon/carbon (RCC) used on the U.S. Space Shuttle's nose and leading edges is an example of this concept [30].

(5) Heat pipe. The heat pipe relies on high effective thermal conductivity (via internal convection), many times greater than that of copper, to move heat from an area of intense, localized heat to cooler locations where it can be effectively rejected from the vehicle's surface or internally stored (e.g. by heating fuel). Several heat pipe concepts have been explored [33], [34], [35], [36], including the development of a heat pipe leading edge for the Space Shuttle Orbiter [33]. 
(6-8) Transpiration cooling, film cooling, convective cooling. These active TPSs use forced convection (coupled with evaporation in the first two) of a liquid coolant for removal of heat. Active cooling systems often add increased complexity and only receive consideration when passive cooling techniques are deemed insufficient.

Because passive concepts suffer from both excessive mass (case 1) and low fracture toughness (case 2), and the added complexity of an active concept is not desirable, we limit further discussion here to the passive concepts of cases 3 to 5; namely ablative, hot structure, and heat pipe TPS, which are most suited for the thermo-mechanical stress state at a hypersonic vehicle's leading edges.

\section{2(a) Ablative TPS}

Figure 2.6 depicts the mechanisms by which heat is shielded from the airframe by an ablative, reinforced polymer (resin) composite. As hot gases behind the boundary layer impinge on the vehicle surface, a polymer is heated and undergoes a pyrolysis reaction. The reaction is endothermic, absorbing heat from the boundary layer and expending it to create a carbonaceous char and reaction gases which diffuse into the boundary layer. The char has a high emissivity and is effective at radiating absorbed heat back into space while the gas byproducts thicken the boundary layer which reduces convective heating. A reinforcement in the polymer, often fibers of silica, serves a dual purpose. First, they hold the char layer to 
the vehicle, preventing its removal by aerodynamic shear forces. This slows the ablation rate of the underlying virgin material by hindering heat ingress. Second, the silica fibers absorb heat as they melt. Silica, in particular, is a highly viscous liquid which remains on the structure, blocking heat, until the aerodynamic forces are sufficient to remove it. When it is torn from the surface, the absorbed heat is removed. Identically, heat is removed when char spalls (mechanical erosion) from the surface [29], [31], [37], [38].

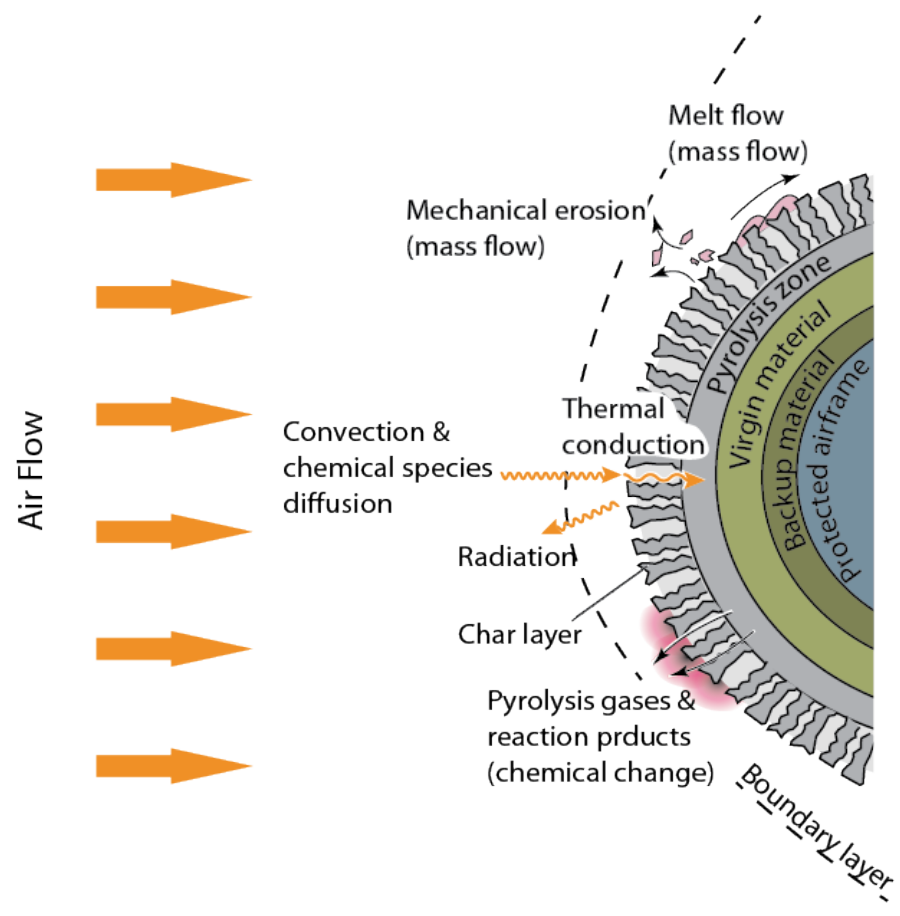

Figure 2.6. The heat dissipation mechanisms of ablation heat shields. Adapted from [31] and [39].

Materials for ablation must have good thermal insulation characteristics to confine the ablation process to the surface, produce gaseous reaction byproducts for boundary layer 
thickening, and be able to endure aerodynamic loads as well as mechanical and thermal shock [29].

Ablatives were first developed in the mid 1950s by the U.S. Army and U.S. Air Force for an InterContinental Ballistic Missile (ICBM) which was to leave Earth's atmosphere and reenter it at high Mach number. The U.S. Army was the first to test a reinforced polymer ablative - a melamine resin reinforced with a glass fiber. Initial success with this material led to further testing of polymers including silicones, phenolics, teflon, epoxies, polyesters, and synthetic rubbers [1]. Filler materials for strength included fibers of silicon dioxide and aluminum silicate, mica, quartz, asbestos, nylon, graphite, beryllium, beryllium oxide, and cotton [1].

Ablative materials may be grouped into one of the following four categories [29]:

(1) Polymers which depolymerize into monomers whose vapor pressure are sufficiently high to flash directly to a gas (e.g. teflon);

(2) Materials which sublime and react with air behind the boundary layer creating a surface combustion product (graphite);

(3) Materials which melt and then vaporize (silicon dioxide); and,

(4) Composite materials which pyrolize and char such as a fiber reinforced epoxybased matrix. 
Composites make use of constituents that dissipate heat and provide thermal resistance to the airframe by several of the mechanisms shared by categories (1)-(3). This multifunctional trait makes them attractive for use on high speed flight vehicles.

The search for advanced ablative materials has mostly been driven by the need for lightweight heat shields on unmanned space vehicles descending into planetary atmospheres other than Earth's. Over the last 30 years (post-Apollo period), ablator material advancements may be classified into two categories, carbon-based Lightweight Ceramic Ablators (LCA) and silicone-based LCAs [40]. Both consist of a fibrous ceramic reinforcement impregnated with an organic resin [41].

A carbon-phenolic LCA system was used on the Pioneer-Venus and Galileo probes whose peak heat fluxes was $4.7 \mathrm{~kW} / \mathrm{cm}^{2}$ and peak pressures reached $300 \mathrm{kPa}$ [40]. Another carbon-based LCA material is being considered for NASA's proposed ORION Crew Exploration Vehicle (CEV). Designed to withstand a heat flux above $300 \mathrm{~W} / \mathrm{cm}^{2}$, the Phenolic Impregnated Carbon Ablator (PICA) consists of a chopped carbon fiber reinforcement infiltrated with a phenol-formaldehyde resin. A high temperature vacuum carbonization step at $780^{\circ} \mathrm{C}$ followed by a heat treatment at $1780^{\circ} \mathrm{C}$ results in a highly porous, all-carbon structure which has low thermal conductivity compared to monolithic graphite and even carbon foam. Depending on processing parameters, final densities are in the $250-600 \mathrm{~kg} / \mathrm{m}^{3}$ range $[41]$. 
The silicone-based LCAs have lower densities than PICA but are designed for less extreme reentry environments (heat fluxes $<300 \mathrm{~W} / \mathrm{cm}^{2}$ ). Silicone Impregnated Reusable Ceramic Ablator (SIRCA), with a density of $150-400 \mathrm{~kg} / \mathrm{m}^{3}$, consists of silica fibers in a vulcanized silicone matrix [42], [43]. It was baselined for the leading edges on the X-34A, a Mach 8 vehicle fueled by liquid rocket propellant which, though built, was never flown [40].

Unlike its hypersonic predecessors, scramjet propelled vehicles impose additional constraints which make an ablative less suited for its TPS. First, a shape change to the coating, a result of its evolving thickness during ablation, can have a significant impact on critical aerosurface dynamics. Second, an ablative is designed for exposure to high heat fluxes for short durations - flight time is dependent on coating thickness - and is suited for atmospheric reentry but not sustained flight. Third, the parasitic weight is detrimental to an airbreathing hypersonic vehicle where a design driver is mass reduction. Fourth, the surface roughness increases drag which, while acceptable for a reentry capsule which requires aerobraking, may pose an issue for a scramjet vehicle. Finally, if access to space is to become economical, the TPS must be reusable.

\section{2(b) Hot structure concepts}

Hot structure design requires lightweight structural materials which can operate at high temperature, have a high surface emissivity for radiative emission of absorbed heat, and 
a capability to withstand the dynamic pressure of hypersonic flight. Figure 2.7 shows a material property chart for specific strength and maximum service temperatures for materials that can be used above $1,000^{\circ} \mathrm{C}$. Here, maximum service temperature defines a temperature above which at which there is significant degradation in performance (due to, for example, solutionizing of a superalloy's strengthening phase(s) or oxidation). The chart shows that high temperature ceramic materials, such as the carbons and oxides are hot structure candidates. Despite the abundance of ceramic materials with high melting temperatures, only a few are capable of maintaining their structural integrity and dimensional stability at temperatures of $2,000^{\circ} \mathrm{C}$ in an aggressive oxidizing environment [44]. Most refractory metallics show good temperature resistance only up to $1,200^{\circ} \mathrm{C}$, although the tantalum alloys have service temperatures past $1,500^{\circ} \mathrm{C}$. Environmental barrier coatings extend the serviceable temperature range of some refractory metallics [45], [46]. 


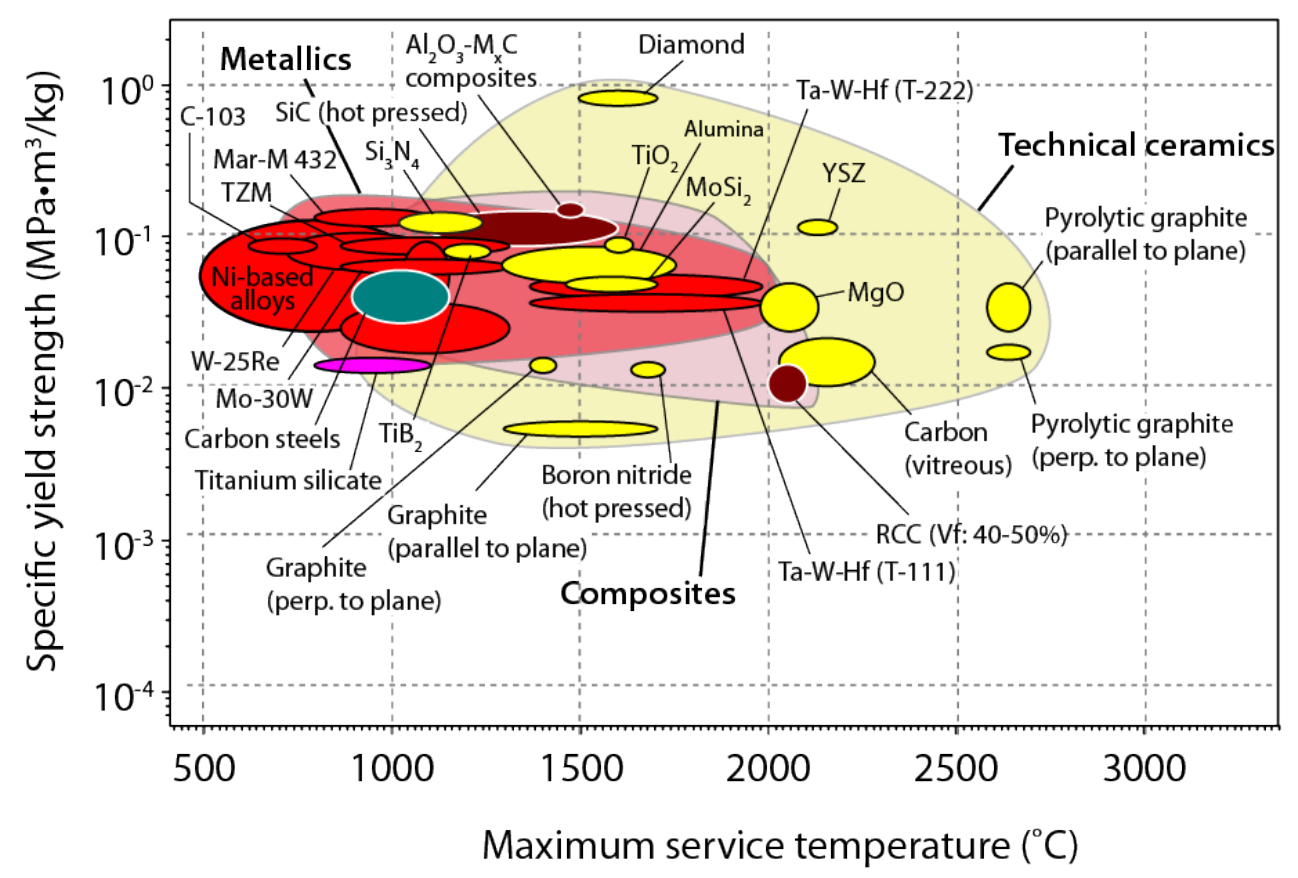

Figure 2.7. The specific yield strength of some high temperature metals and ceramics.

The X-15 research vehicle was the first hypersonic aircraft to make use of a hot structure TPS [1]. Inconel X (now more commonly referred to as X-750), a nickelchromium-based superalloy with $\gamma^{\prime}$ phase strengthening, maintains $90 \%$ of its room temperature yield strength at $600^{\circ} \mathrm{C}[47]$, so it could be used to bear structural loads while the metallic nature of the material permitted spreading of localized hot spots. The uncoated alloy was successfully used for the outer skin of the X-15 for low Mach number flights, but required an ablative coating for flights above Mach 6 [48].

An example of one of the most successful and yet, at the same time, spectacular failures of a hypersonic hot structure could be found on the U.S. Space Shuttle, which flew a 
total of 133 safe flights [49]. The Shuttle leading edge TPS comprises a high temperature Reinforced Carbon/Carbon (RCC) ceramic. It is manufactured by pyrolizing a nylon cloth (converting it to graphite) which is then impregnated with a phenolic resin and cured in an autoclave. The cured material is pyrolized again to transform the cured resin to carbon. The part is then densified by impregnating it with furfural alcohol in a vacuum and pyrolizing a third time for carbon transformation. The densification step is repeated three times until the final RCC part has achieved a density of $1,440-1,600 \mathrm{~kg} / \mathrm{m} 3$ [30]. The RCC leading edges are exposed to peak temperatures of $1,310^{\circ} \mathrm{C}$ on reentry and exhibit low thermal expansion [11]. Failure occurred as a result of the material's low fracture toughness $(6 \mathrm{MPa} \sqrt{\mathrm{m}},[50])$ when impacted by high velocity foam which detached from the Shuttle's fuel tank[51].

One of the most successfully tested airbreathing research vehicles was the X-43A developed under NASA's HyperX program, Figure 2.8. In 2004, the vehicle completed Mach 7 and Mach 10 flights. The TPS consisted of a coated RCC on its nose leading edge which had a $0.763 \mathrm{~mm}$ radius. The RCC used an unbalanced continuous carbon fiber weave of 3:1 to increase the density of high thermal conductivity fibers oriented perpendicular to the spanwise direction for effective transport of the heat from the tip to the flat portions of the vehicle where it could be radiated to the ambient. A higher weave ratio was considered; however, a balance of fiber orientation was needed to resist the compressive stresses formed by restrained thermal expansion in the spanwise direction [52]. 


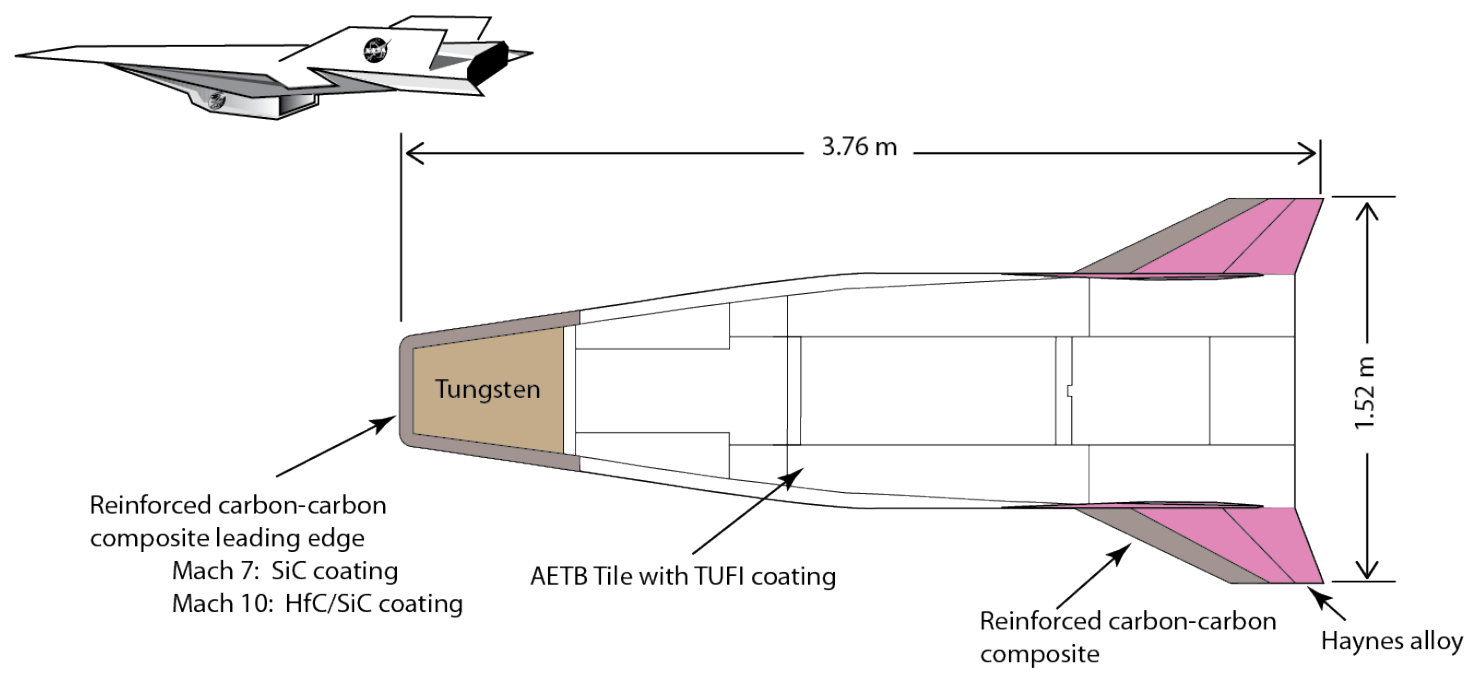

Figure 2.8. TPS for NASA's X-43A experimental vehicle. Adapted from [32].

A significant drawback to RCC is its poor oxidation resistance at temperatures as low as $500^{\circ} \mathrm{C}$. The gaseous oxidation reaction products $\mathrm{CO}$ and $\mathrm{CO}_{2}$ result in erosion and rapid mass loss [53]. In the case of the Shuttle leading edge, a SiC coating was applied via a high temperature diffusion process with an alumina, silicon, and silicon-carbide dry pack [30]. Oxidation of the $\mathrm{SiC}$ surface then leads to the initial evolution of $\mathrm{CO}$ quickly followed by formation of $\mathrm{S} \mathrm{SiO}_{2}$ barrier layer that seals the surface. Further oxidation requires transport through the $\mathrm{SiO}_{2}$, which is slow [54]. Thermal expansion mismatch between the $\mathrm{SiC}$ layer and RCC substrate results in cracking of the SiC layer [30]. Tetraethylortho silicate (TEOS) is therefore vacuum impregnated and pyrolyzed to seal the cracks in the SiC layer [30]. Figure 2.9 shows the layers of an advanced RCC material that incorporates an additional coating of sodium-silicate glass for further oxidation resistance [53], [55]. 


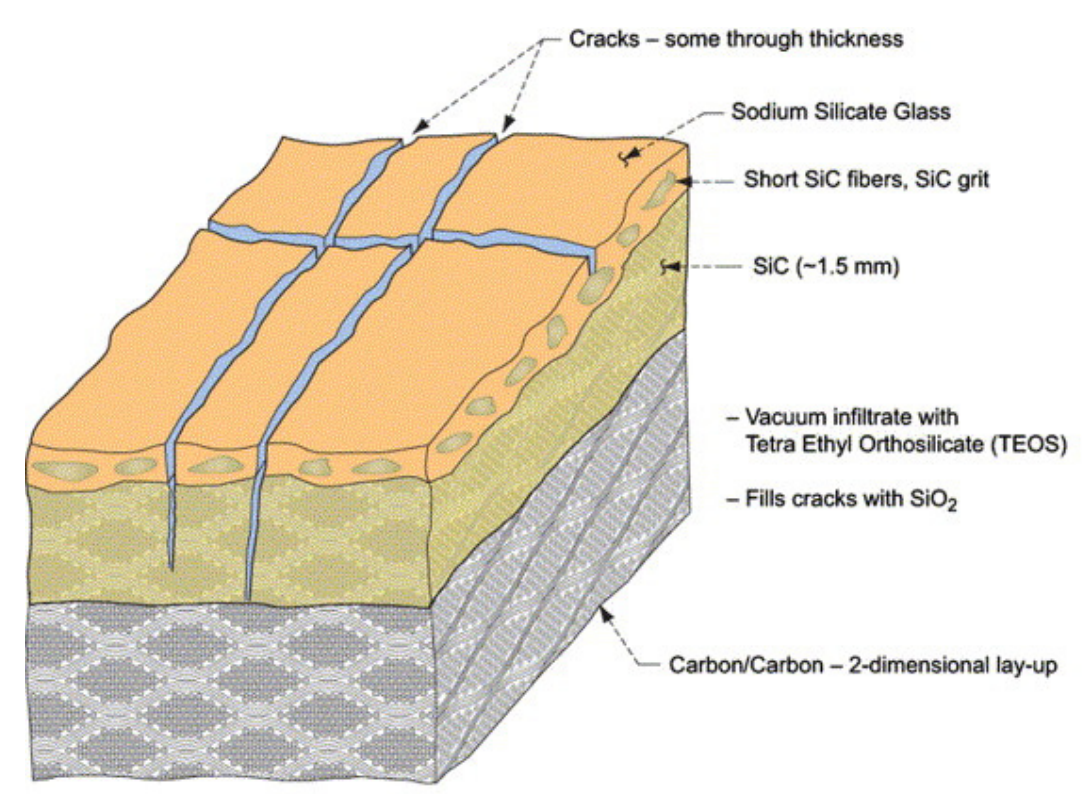

Figure 2.9. Advanced RCC with oxidation protection coating [53].

Presently, structural materials that reliably operate in a high temperature oxidizing environment are limited to RCC (with $\mathrm{SiC}$ or $\mathrm{HfC}$ coatings), $\mathrm{SiC}, \mathrm{Si}_{3} \mathrm{~N}_{4}$, oxide ceramics, and their composites [56]. The formation of a protective layer of $\mathrm{SiO}_{2}$ protects $\mathrm{Si}$-based materials and coatings from further oxidation under conditions of moderate temperature (up to $\approx 1600^{\circ} \mathrm{C}$ ) [57]. For both $\mathrm{SiC}$ and $\mathrm{Si}_{3} \mathrm{~N}_{4}$, Vaughn and Maahs found the transition from passive oxidation protection to gaseous production of $\mathrm{SiO}$ (and mass loss) occurred at conditions of $1,347^{\circ} \mathrm{C}$ for an oxygen partial pressure of $2.5 \mathrm{~Pa}$ and $1,543^{\circ} \mathrm{C}$ at $123.2 \mathrm{~Pa}$, indicating that increases in oxygen pressure elevated the maximum use temperature of the binary compounds [58]. Scramjet engines typically operate with dynamic pressures in the range of $24 \mathrm{kPa}$ to $96 \mathrm{kPa}$ [23]. The mole fraction of oxygen in air is 0.21 and stays relatively 
constant with altitude. Therefore, oxygen partial pressures in stagnation regions can be estimated between $5.04 \mathrm{kPa}$ and $20.16 \mathrm{kPa}$, leaving the Si-based compounds susceptible to oxidation at scramjet leading edge operating temperatures. Boron-doped RCC was shown to improve the oxidation resistance (raising initial oxidation temperatures form $550 \mathrm{C}$ to $700^{\circ} \mathrm{C}$ [59]) through the formation of a passive boron oxide coating and the introduction of substitutional atoms in the carbon lattice which suppressed $\mathrm{O}_{2}$ chemisorption [60], [61], [62].

High temperature oxide ceramics, such as zirconia $\left(\mathrm{ZrO}_{2}\right)$ and halfnium $\left(\mathrm{HfO}_{2}\right)$, have melting points of $2,700^{\circ} \mathrm{C}$ and $2,800^{\circ} \mathrm{C}$, respectively [56]. Yet, compared to the $\mathrm{SiC}$ and $\mathrm{Si}_{3} \mathrm{~N}_{4}$, their creep rates are high [56]. Zirconia undergoes crystallographic transformations at high temperatures which are accompanied by volume changes which can cause fracture upon cooling. To improve its toughness, yttrium has been used in amounts of $6-8 \mathrm{wt} \%$ to stabilize zirconia in a tetragonal phase [63]. However, this is only successful for heating to $1,300^{\circ} \mathrm{C}$ [63], [64], [65], well below the leading edge temperatures of interest.

Hot pressed UltraHigh Temperature Ceramics (UHTC), which consist of rare earth and refractory metal borides and carbides, have been most recently considered for use as an advanced hot structure TPS due to their combination of high melting point and high thermal conductivity. $\mathrm{HfB}_{2}, \mathrm{ZrB}_{2}, \mathrm{HfC}$, and $\mathrm{ZrC}$ have melting points in excess of $2,500^{\circ} \mathrm{C}$ [44]. $\mathrm{HfB}_{2}$ and $\mathrm{ZrB}_{2}$ have much higher thermal conductivities than the carbides, suggesting they may be suitable materials for leading edges where thermal gradients can be extreme [44]. 
The room temperature thermal conductivity of $\mathrm{HfB}_{2}$ has been measured at over $70 \mathrm{~W} / \mathrm{mK}$ at $800^{\circ} \mathrm{C}$ (compared to $20 \mathrm{~W} / \mathrm{mK}$ at room temperature for $\mathrm{HfC}$ ) [57], but is sensitive to the microstructure formed during hot pressing [56].

While the thermal properties are promising, the mechanical properties of the diborides are often problematic, with $\mathrm{HfB}_{2}$ and $\mathrm{ZrB}_{2}$ exhibiting poor fracture toughness [66]. The fracture strength, $\sigma_{f}$, of a ceramic is a function of the fracture toughness, $K_{I C}$, and the defect radius, $a$;

$$
\sigma_{f}=Y K_{I C} / \sqrt{\pi a}
$$

where $Y$ is a geometric factor. The fracture strength can be improved by either increasing the fracture toughness or reducing the defect size [67]. Toughening by grain refinement and the addition of particulate reinforcement, such as $\mathrm{SiC}, \mathrm{MoSi}_{2}$ or $\mathrm{ZrSi} i_{2}$, have been explored to impede the propagation of cracks (by deflection) [56].

The high temperature metallic diborides also exhibit better oxidation resistance then RCC[68]. When $\mathrm{HfB}_{2}$ and $\mathrm{ZrB}_{2}$ are pressed with a silica former, typically $10-30 \% \mathrm{SiC}$ [44], a protective layer of $\mathrm{SiO}_{2}$ is formed that persists up to $1,800^{\circ} \mathrm{C}$ [69]. Peng et al. found the addition of $\mathrm{TaSi}_{2}$ improved oxidation resistance up to $1,550^{\circ} \mathrm{C}$ due to the formation of a denser $\mathrm{SiO}_{2}$ layer which inhibits the inward diffusion of oxygen [70]. Zhang et al. found the oxidation resistance could be improved to $2,400^{\circ} \mathrm{C}$ through the addition of lanthanum hexaboride (LaB6). Upon exposure to a high temperature oxidizing environment, a 
protective passive lanthanum zirconate $\left(\mathrm{La}_{2} \mathrm{Zr}_{2} \mathrm{O}_{7}\right)$ coating formed on its surface, covering cracks and protecting subsurface material from oxygen exposure [71]. A follow-up study on the mechanical properties of $\mathrm{ZrB}_{2}-20 \% \mathrm{vol} \mathrm{SiC}-10 \% \mathrm{vol} \mathrm{LaB}_{6}$ reported a fracture toughness of 5.7MPa $\cdot \mathrm{m}^{1 / 2}$, compared to reports of $\mathrm{ZrB}^{2}-20 \% \mathrm{vol} \mathrm{SiC}$ at $4.0-4.8 \mathrm{MPa} \cdot \mathrm{m}^{1 / 2}[69]$.

The impact tolerance (fracture toughness) of TPS designs is critical to their performance. Should a fracture compromise their surface, hot gases could penetrate to vehicle structural components or systems not capable of withstanding elevated temperatures. In the case of the Columbia shuttle disaster, a piece of foam from the External Tank detached and struck, at a relative speed of $237 \mathrm{~m} / \mathrm{s}$, the RCC leading edge of the left wing. The breach, reproduced in laboratory tests and shown in Figure 2.10, allowed superheated gases to penetrate the TPS and melt the aluminum airframe. As a result of the accident, the Columbia Accident Investigation Board recommended the initiation of "a program designed to increase the Orbiter's ability to sustain minor debris damage by measures such as improved impact-resistant RCC...” [51]. 


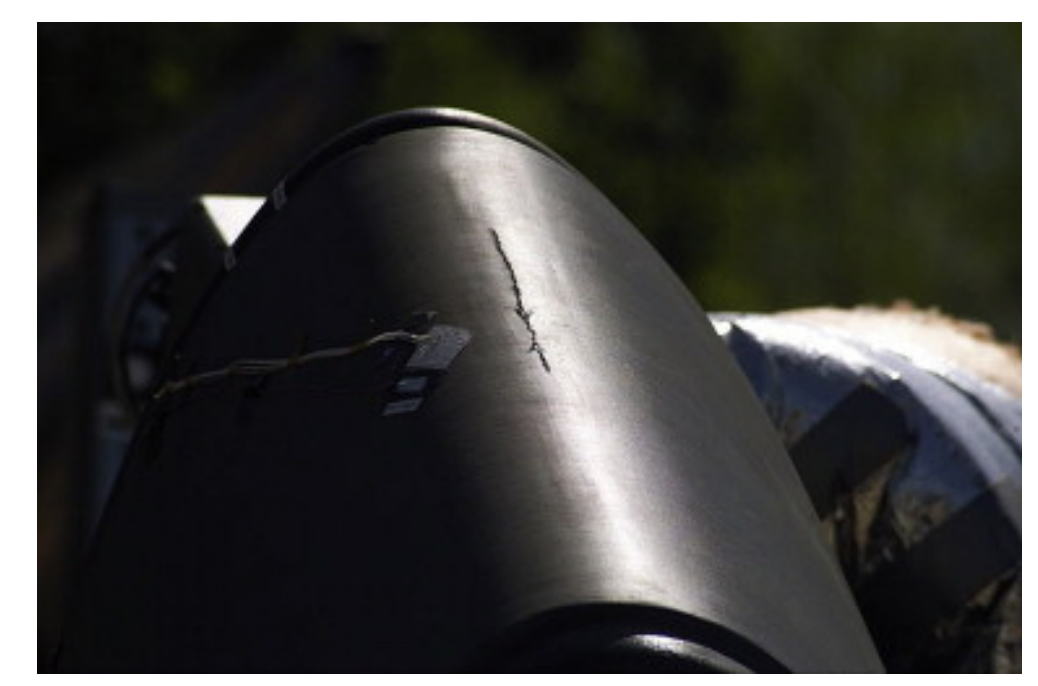

Figure 2.10. Crack in a RCC Shuttle leading edge from a laboratory-controlled foam strike [51].

\section{2(c) Heat pipe TPS}

It was shown earlier (c.f. Figure 2.1 and Figure 2.7) that refractory metallics are generally limited to use temperatures below $1,200^{\circ} \mathrm{C}$ with the exception of some tantalumbased alloys. If metallics are to be used at hypersonic leading edges, either their high temperature thermo-mechanical properties must be improved or their operating temperatures must be kept below this limit. On the latter point, an alternative leading edge metallic TPS concept based on high thermal conductance heat pipes has been proposed [613]. A heat pipe is a two-phase, passive heat transfer device capable of transporting, (by more than an order of magnitude) the heat that can be thermally conducted by metals. Heat pipes have been used in applications ranging from spacecraft thermal control to electronics 
packaging to permafrost preservation under the Alaskan oil pipeline [72]. Most laptop computers utilize heat pipes for cooling [73].

Figure 2.11 schematically illustrates the fundamental operating mechanisms of a heat pipe. The concept is based upon the evaporation of a fluid near a heat source that sets up a region of elevated pressure in the sealed system. The latent heat of evaporation is transported down the pressure gradient as a vapor stream until it condenses at cooler regions in the device. There it releases the latent heat of vaporization at a location well suited for removal. Replenishment of the condensed working fluid to the evaporator region is driven by a liquid pressure gradient in a saturated, porous wick which lines its walls. The evaporation, vapor flow (heat transport), and condensation of the working fluid isothermalizes the structure.

Through proper material selection and design, extremely large heat fluxes can be driven down a heat pipe with only small temperature drops along its length; effective thermal conductivities can reach several thousand $\mathrm{W} / \mathrm{mK}$ [72], [73], [74], an order of magnitude larger than that of copper. It is this extremely high "effective" thermal conductivity that is attractive for a TPS application in the hypersonic environment. The rapid spreading of heat from the stagnation point could reduce peak temperatures and thermal gradients (and therefore thermal stresses). Because the case material could be a metal, the TPS may be much less vulnerable to impact fracture. 


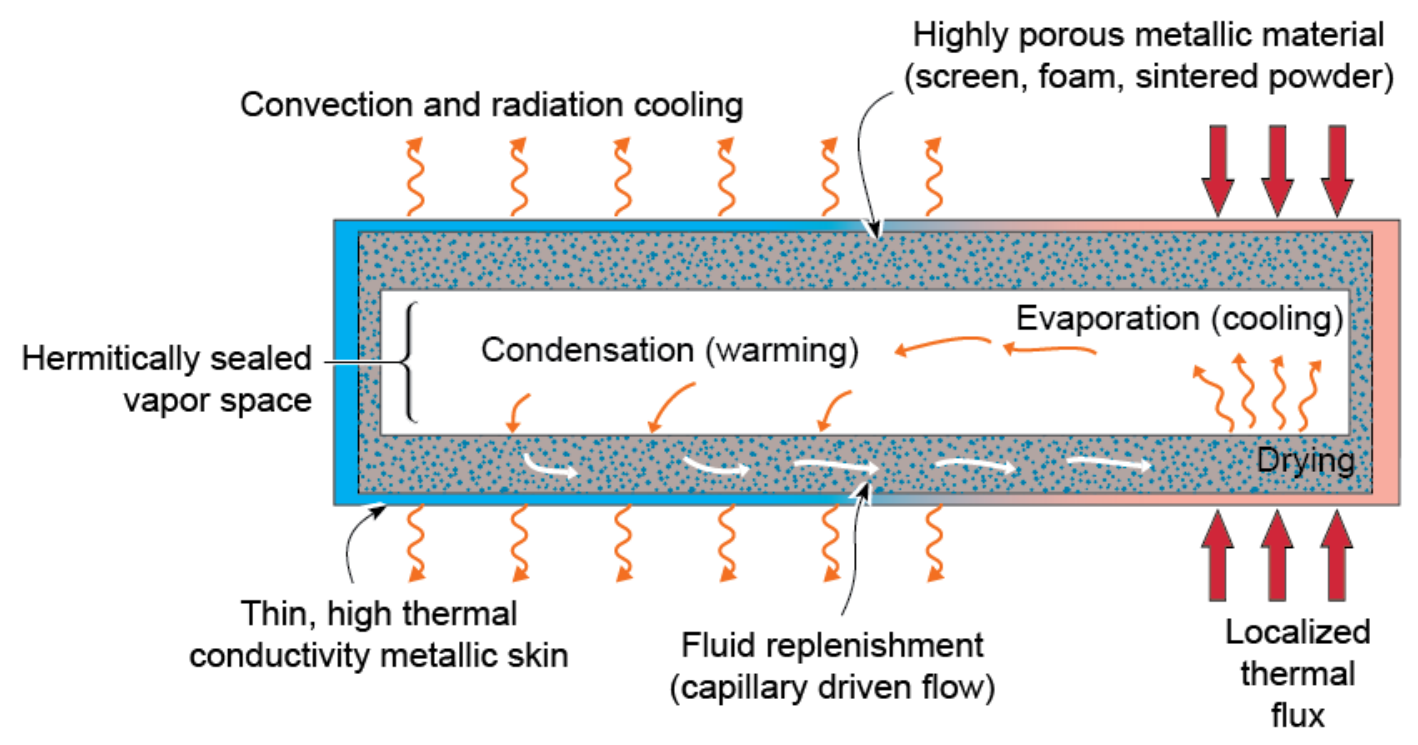

Figure 2.11. Cross-section schematic of a cylindrical heat pipe illustrating its operation.

The concept of coupling heat pipes to leading edges has received only limited investigation [34], [35], [36], [75], [76]. Nearly all leading edge heat pipe concepts employed arrays of hollow circular tubes or channels either metallurgically joined to a high temperature skin or embedded in a wedge-shaped composite [33], [34], [35], [36]. The heat pipes were bent to conform to the radius of the leading edge. Evaporation occurs in the radius of the bend, driving vapor in opposing directions aft of the tip where it condenses, releasing its latent heat for conduction through the tube wall and skin from which it is radiated into space. 
(a)

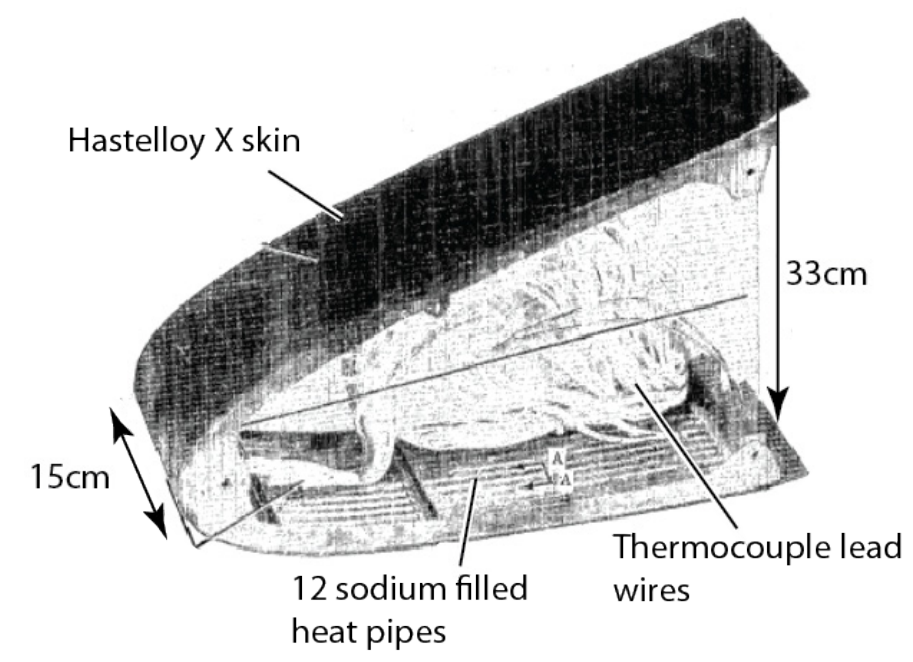

(b)

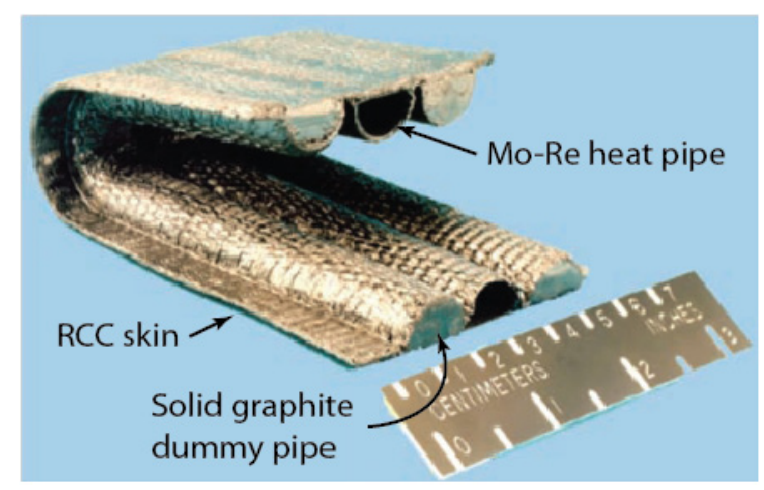

Figure 2.12. (a) An Orbiter leading edge developed by NASA using a Hastelloy X and sodium working fluid [33], and (b) a Mo-Re heat pipe embedded in a RCC leading edge skin [35].

Carmada and Masek designed and tested a Space Shuttle Orbiter leading edge using twelve Hastelloy $\mathrm{X}$ heat pipes metallurgically joined using a nickel-braze alloy to an outer Hastelloy X skin, shown in Figure 2.12(a). Sodium was used as the working fluid. The half 
-scale test model with an estimated ${ }^{4}$ radius on the order of $10 \mathrm{~cm}$ was designed to sustain a cold wall heat flux of $40.8 \mathrm{~W} / \mathrm{cm}^{2}$ and reduce wall temperatures at the tip from $1,317^{\circ} \mathrm{C}$ to $1,012^{\circ} \mathrm{C}$. Radiant heat tests to simulate the high enthalpy Shuttle Orbiter reentry environment showed a temperature difference of only $5.6^{\circ} \mathrm{C}$ over the $56 \mathrm{~cm}$ length [33], [34].

A slightly different approach has been taken by others, replacing the conventional tubes with an improved "D-shaped" cross-section [75], [76], Figure 2.12(b). Positioning the flattened side of the heat pipe wall against the outer skin reduces the thermal pathway for heat ingress and egress. Clark and Glenn designed and tested a Haynes 188 D-shaped heat pipe with a sodium working fluid and nickel screen wick. The tube was formed by bending to replicate a generic leading edge profile but was not adhered to a skin. A leading edge radius was not specified. Relatively low temperature tests showed maximum wall temperatures at the tip of $600^{\circ} \mathrm{C}$ and a maximum temperature difference over the $175 \mathrm{~cm}$ length (active portion) of approximately $175^{\circ} \mathrm{C}$ [76]. A second leading edge having multiple "D-shaped" cross-section tubes with a sintered powder wick lining it's walls was fabricated from a high temperature niobium-based alloy, C-103 (Nb-10Hf-1Ti), and adhered to a skin of the same alloy which had been formed to a leading radius of $2.22 \mathrm{~cm}$. This specimen was neither charged nor tested [76]. Glass et al. embedded Mo-41Re "D-shaped" heat pipes in a 3D woven carbon perform, shown in Figure 2.12(b), which was densified to form a reinforced carbon-carbon leading edge with a $1.27 \mathrm{~cm}$ radius [35]. The tubes had a 400

\footnotetext{
${ }^{4}$ Radius dimension was estimated from photographs of the test article.
} 
mesh Mo-5Re screen wick and used lithium as a working fluid. Four tests were conducted on the test article before catastrophic failure due to the formation of an electrical arc between the test article and an RF induction heater. Neither design temperatures nor isothermal operation were obtained, with a temperature difference across the test article of at least $200^{\circ} \mathrm{C}$ at steady state [75].

There are a number of drawbacks to this heat pipe array concept. Firstly, fabrication issues with bending the tubes to a small leading edge radius (on the order of $3 \mathrm{~mm}$ or less for scramjet inlets) is problematic [75]. At small radii, buckling of the wall at the bend line weakens the tube and pinches the vapor space closed, restricting flow. Secondly, the joining of an exterior face sheet to the heat pipe tubes increases the solid wall thickness. It is optimal to keep this wall thickness as small as possible (subject to any structural requirements) in order to maximize the thermal flux that enters the heat pipe and to minimize high temperatures and induced thermal stresses. Thirdly, in each of the aforementioned systems, it was necessary to install the wick, whether it was sintered powder or a metal screen, prior to forming. During bending, tensile and compressive stresses form in the wick which may cause damage that hinders capillary flow and leads to a dryout condition, or temperature spike, and failure of the heat pipe system.

Incorporating periodic cellular lattices (see Figure 2.13) in a leading edge heat plate design may overcome the aforementioned drawbacks. When configured as the cores of sandwich panels, cellular lattices can exhibit exceptional structural efficiency [77], [78]. The 
mechanical properties of low relative density honeycomb (hexagonal, square, triangular types), truss (pyramidal, tetrahedral, Kagome types), and corrugated (triangular, diamond, NavTruss types) cores are well characterized, having been assessed in compression, shear, and bending, as well under conditions of high impulse loading [79], [80], [81], [82]. These cellular structures potentially overcome several of the disadvantages of past leading edge heat spreaders while improving the structural efficiency of the TPS. This latter aspect is important as the stagnation pressure, skin friction, and acoustic loads can be significant during low altitude hypersonic flight [4].

Truss

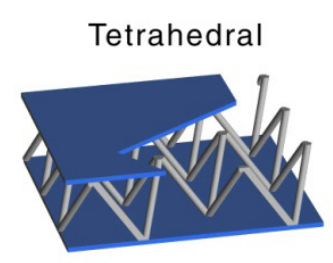

Pyramidal

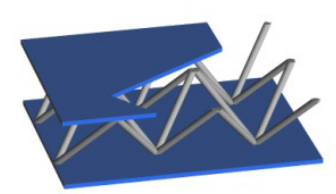

Kagomé

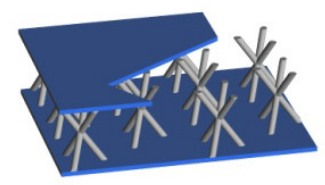

Honeycomb

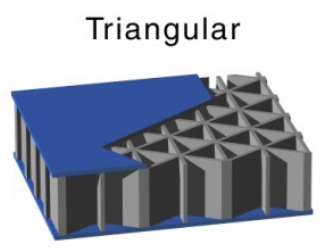

Square

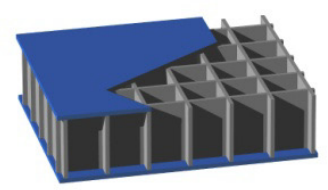

Hexagonal

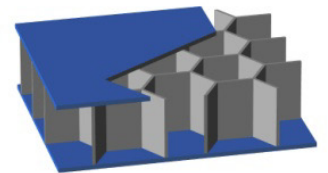

Prismatic

Triangular

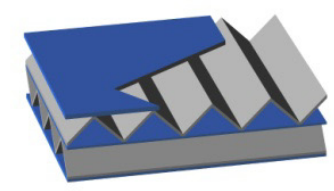

Diamond

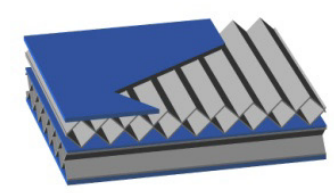

Navtruss

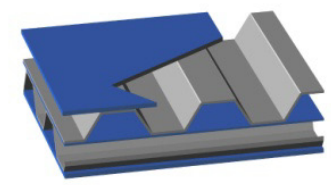

Figure 2.13. Examples of periodic cellular materials. From [78]. 
Several groups have noted the good combination of structural and thermal properties of open-cell lattice or prismatic materials that are well suited for heat pipe structures. Basiulis and Camarda constructed and tested an Inconel 718 hexagonal honeycomb sandwich panel having a metallic woven mesh wick and a potassium working fluid [83]. The honeycomb core was perforated to allow vapor cross-flow between cells and notched at the base of its walls for fluid return. Queheillalt et al. designed and tested a nickel-plated aluminum flat heat plate having a truncated square honeycomb core, which allowed three dimensional vapor flow [84]. A nickel foam was used for the wick and deionized water for the working fluid. The nickel coating was used to avoid hydrogen generation through a reaction between the aluminum and water working fluid. This structure formed the basis for a large (6ft by $14 \mathrm{ft})$ jet blast deflector [85].

An I-core sandwich panel topology has been proposed for a high temperature leading heat edge pipe [86], [87], [88], Figure 2.145. The design differs from the cylindrical and "D-shaped" concepts by employing an I-core sandwich panel with rectangular channels situated in the forward-aft direction. The proposed manufacturing process involves fabricating individual, sealed channels which are cold-formed into the leading edge curvature and then metallurgically joined at their sidewalls. Merrigan et al. evaluated a Hastelloy X leading edge heat pipe of this design with a sodium charge [86]. Only a single rectangular channel was tested. The tip radius was $5.08 \mathrm{~cm}$ while the rectangular channel had internal

${ }^{5}$ A photograph of reproducible quality could not be found for the leading edge systems cited here. 
dimensions of $1.27 \mathrm{~cm}$ by $0.953 \mathrm{~cm}$ and used a stainless-steel mesh wick. During thermal testing there was a breach of the wall material due to overheating. The cause was attributed to dryout in the wick at the location of a tear which formed during bending. Boman and Elias [87] designed and tested an identical system. An attempt was made to internally instrument the vapor space with pressure transducers for validating model predictions of vapor thermodynamics. This system also failed; it was hypothesized that this was due to inhomogeneous distribution of solid phase sodium in the wick at the start of the test [87].

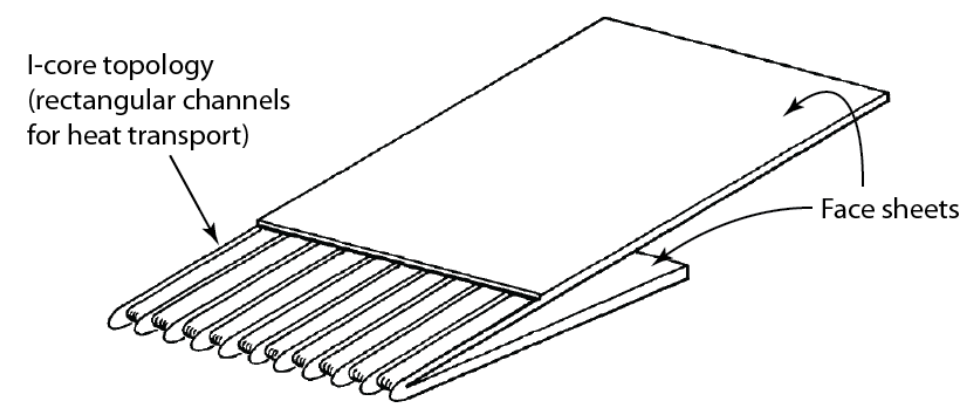

Figure 2.14. Leading edge I-core system which requires bending to form the leading edge tip radius [88].

These systems use a constant cross-sectional area I-core, requiring the panel to be formed, by bending, into the leading edge shape in a manner similar to the heat pipe approach; therefore, the above noted disadvantages of using pipes pertain to this I-core design as well. 


\subsection{Proposed leading edge heat spreader}

In order to overcome these drawbacks, a structural leading edge heat spreader is proposed which, in contrast to other heat pipe TPS systems, requires no bending after heat pipe fabrication. The prismatic and truss cores of the leading edge concepts in Figure 2.15 provide structural support and configure a pathway for easy vapor flow. Its core extends between the top and bottom skin regions to support the entire leading edge under hypersonic loads, with much higher bending stiffness and compressive strength than other designs. One can envisage an open cell truss core design as well. The all-metallic heat spreader concepts use a diverging vapor space whose cross-section increases from the stagnation area at the tip to regions aft. Leading edge heat spreaders of this type have not received attention as a prospective TPS. 
(a)

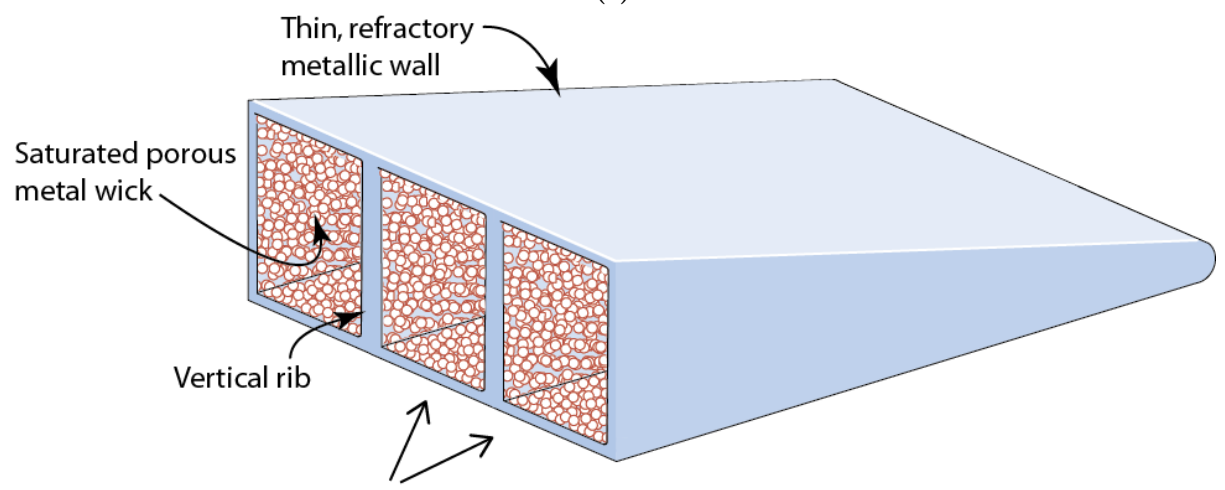

Multiple vapor cells

(b)

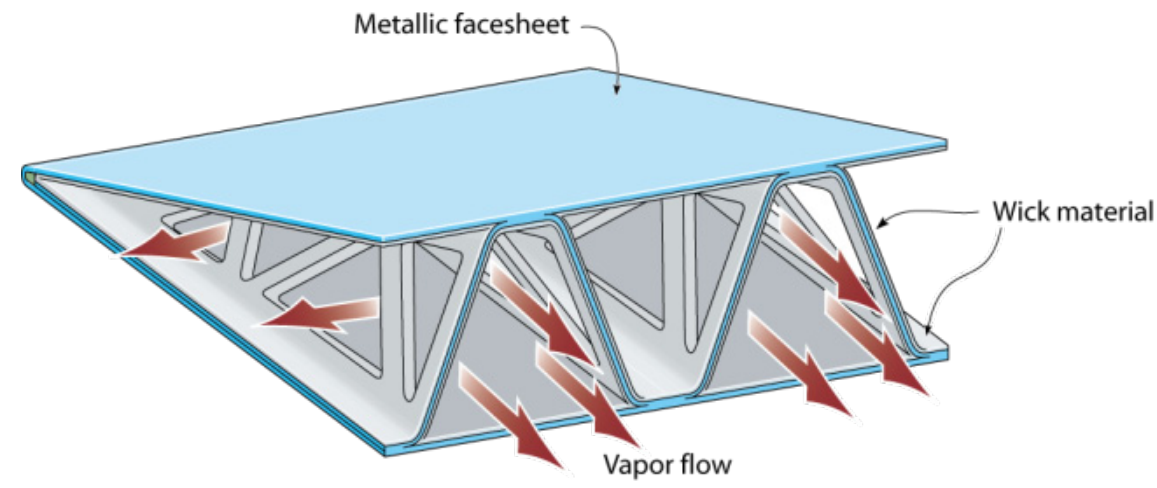

(c)

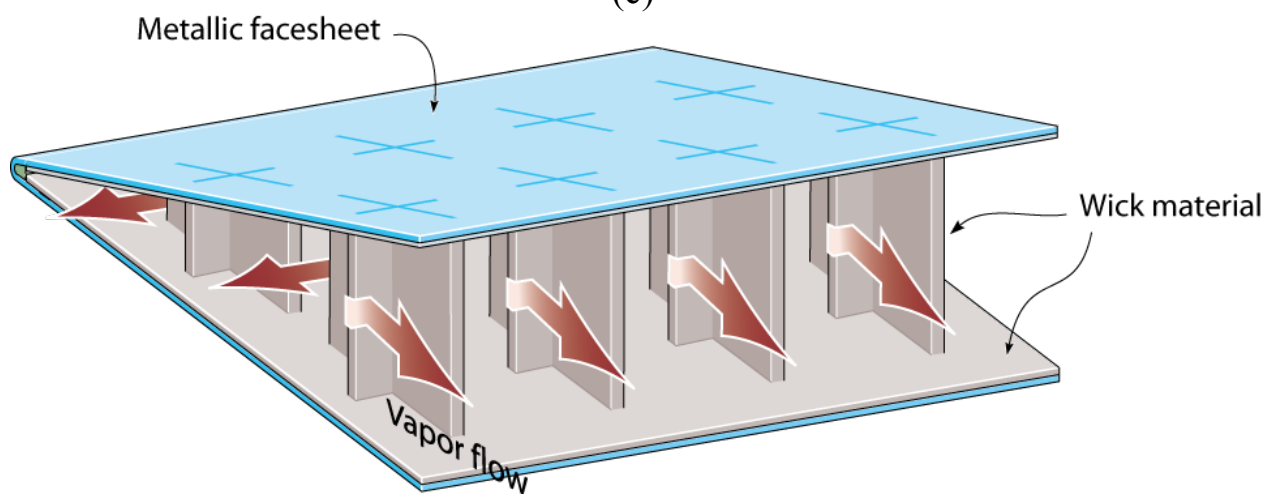

Figure 2.15. Three leading edge heat spreader concepts: (a) an I-core design having low flow resistance in the longitudinal direction, (b) a perforated corrugation design and (b) cruciform (truncated honeycomb) design, both of which allow longitudinal and transverse vapor flow. 
Figure 2.16 shows a number of different heat transfer fluids which have been used in heat pipes. Each bar is bound by its melting temperature on the left side and its critical temperature on the right. For heat pipe operation, the fluid must exist as a two-phase medium. The red region outlines the operating temperatures of interest for hypersonic applications. The alkali metals, which have a very high latent heat of evaporation (see Appendix B) are optimal for this application. However, selection of a compatible case material is limited due to the highly reactive nature of the liquid alkalis. Liquid metal corrosion is a significant concern, even for short duration flight (less than 10 minutes). Despite a lack of consensus on the particular corrosion mechanisms, there is conclusive evidence for good chemical compatibility between specific liquid metal and containment material combinations [89], [90], [91]. This dissertation investigates compatible material combinations Ni-based Inconel 718 (IN718) and sodium, niobium-based C103 and lithium, and molybdenum-based TZM and lithium. 


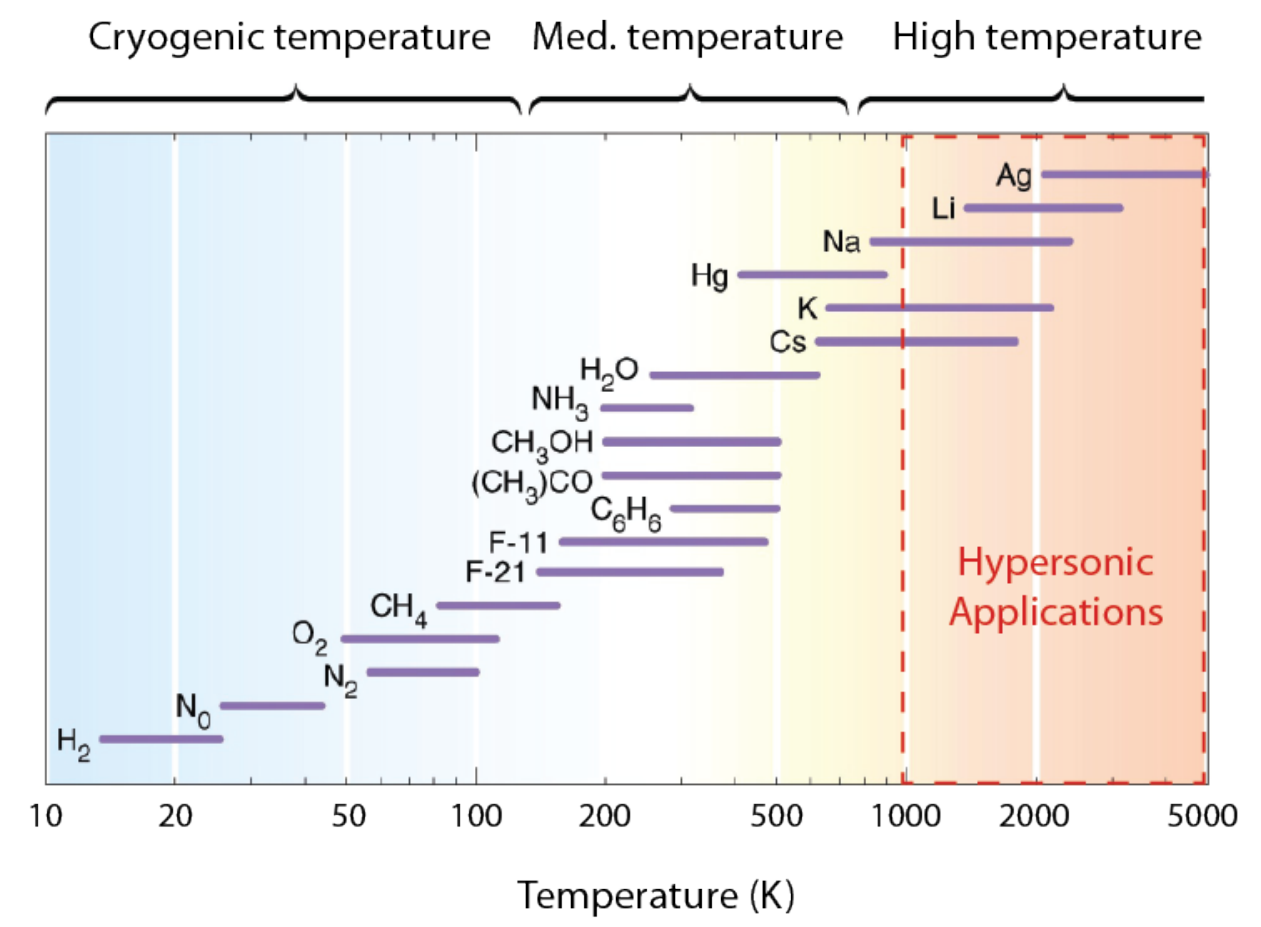

Figure 2.16. Thermal operating window of some heat pipe working fluids [72].

The proposed wedge heat spreader can be employed anywhere on the vehicle with localized heating at the tip and connectivity to cooler regions, see Figure 1.2. This dissertation explores the use of leading edges at scramjet inlets whose aerodynamic design requirements require a leading edge radius of $\sim 3 \mathrm{~mm}$ and a wedge half-angle of $6^{\circ}$. All subsequent analysis assumes these values.

Before undertaking a design analysis, a more rigorous theory of heat pipe operation in the thermal hypersonic environment is necessary. This is developed in the next chapter. 


\section{Chapter 3. Leading edge design methodology using hypersonic flow theory}

The hypersonic heat flux model presented in Chapter 2 (see equations (2.1) and (2.2)) are insufficient for even first-order analysis of a potential leading edge thermal protection concept. The model, and equations that represent it, do not include wall temperature effects. The exclusion of the wall temperature as a variable in any heat spreader model would be a significant flaw since it eliminates the possibility of using it as a metric. A model that incorporates a more rigorous description of the heat transfer between the wall and the boundary layer is therefore necessary and is presented here. A coupled flow-wall temperature model is presented to obtain the maximum operating temperature and thermal stresses in a wedge-shaped heat pipe-based leading edge structure. A methodology for their design is then presented which uses hypersonic flow theory to construct design maps which relate design parameters of the leading edge (overall length, wall thickness, and alloy) to its 
operating conditions (isothermal temperature, maximum temperature, maximum thermal stress). The maps are similar to those used for the design of finned heat exchangers to relate design variables (fin length and thickness) to fin efficiency (a measure of heat transfer effectiveness for a particular fin profile). Three potential material systems (case and working fluid combinations) are examined:- (i) a nickel-based with sodium, (ii) a niobium-based alloy with lithium, and (iii) a molybdenum-based alloy with lithium. Their performance over a range of hypersonic Mach numbers is assessed.

\subsection{Background}

As a vehicle flies through the atmosphere, air molecules impact its leading edge surface and a region of stagnated flow develops. This interaction leads to a transfer of some of the vehicle's kinetic energy to potential energy of the gas (i.e. an increase in air pressure near the vehicle surface), to kinetic energy of the gas (i.e. acceleration or deceleration of the gas flow), and for high-speed flight, significant changes to the internal energy of the gas atoms and molecules by rotational/vibrational and electronic excitations. The latter phenomena lead to very high gas temperatures immediately in front of the vehicle leading edge surface, providing a thermal source which transfers heat to the cooler vehicle walls. A second thermal flux contribution can arise from the shear (viscous) resistance of the fluid flow and impacts locations aft of the stagnation point. 
At steady state, a local thermal energy balance must exist between the vehicle and its environment. The heat flux penetrating the vehicle's wall, $q_{w}$, is equal to the net energy transferred by contact with the gas minus that reradiated by the vehicle surface;

$$
q_{w}=q_{g w}+q_{r}
$$

where $q_{g w}$ is the heat transferred from the gas to the wall surface, and $q_{r}$ is the thermal radiation emitted from the wall surface into the gas. This balance is depicted in Figure 3.1.

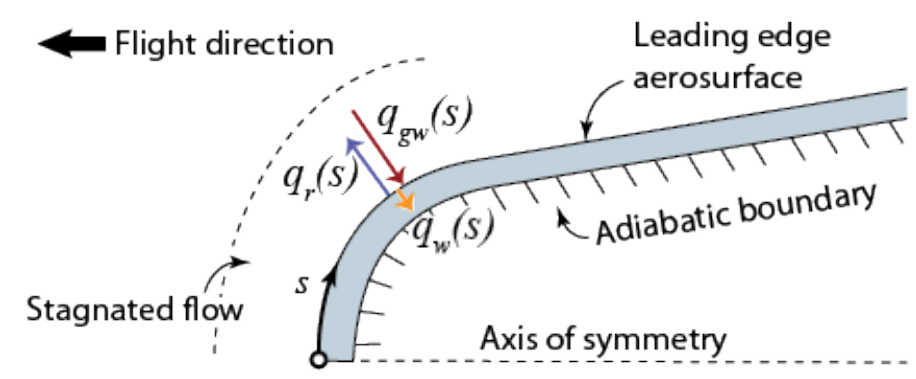

Figure 3.1. Local balance of the heat fluxes at a leading edge surface. The positive flux direction is into the vehicle and " $s$ " is a coordinate on the leading edge surface.

Two limiting cases are used in conventional analysis of the energy balance [4]:

(i) Cold-wall (non-radiating) limit where $q_{r}=0$ and $q_{w}=q_{g w}$. The wall temperature is then assumed to be a cold surface with no radiation cooling. The heat flux into the wall balances the net impinging heat flux. 
(ii) Radiation-adiabatic wall limit where $q_{w}=0$ and $q_{r}=-q_{g w}$. Passive radiation cooling balances the impinging heat flux; the wall temperature is taken to be that of the radiation adiabatic temperature and no heat is conducted into the vehicle's surface.

The first limit was used in the engineering hypersonic heat flux formulas presented in Chapter 2 and while helpful in developing a conservative estimate of the wall heat flux, it provides no means to infer the temperature of the wall. The second limit is valuable for analysis of thermally insulated hot structures in which the wall heat flux is low. However, this does not apply to leading edge heat spreaders which use heat redistribution to control the wall temperature when a high wall heat flux enters the leading edge.

To overcome these limitations, a method for determining the heat flux through the wall is developed below. The model initially assumes that the wall temperature, $T_{w}(s)$, is uniform over the surface and equal to an isothermal wall temperature, $T_{w}(s)=T_{i s o}$. The validity of this assumption can be assessed by examination of the Biot modulus [15], Bi; a dimensionless ratio of a body's conductive thermal resistance to its convective thermal resistance at it surface [15];

$$
\mathrm{Bi}=h D / k
$$

where $h$ is the surface heat transfer coefficient, $k$ is the thermal conductivity of the material, and $D$ is a characteristic dimension, typically the volume of the body divided by its surface area. In general, material systems with a $\mathrm{Bi}<1$ are thermally simple with a nearly 
homogenous internal temperature distribution [15]. The vapor transport in a well designed heat pipe has an effective thermal conductivity $k$ which is orders of magnitude greater than convective transport to the surface $(h D)$. As a result, $\mathrm{Bi} \ll 1$, supporting the isothermal assumption [73], [92].

The free-stream Mach number $M_{\infty}$ is used to define the hypersonic flight window: $M_{\infty} \geq 5$. The Mach number is the ratio of the velocity of an object $V_{\infty}$ relative to the speed of sound, $c$, in the surrounding medium [93];

$$
M_{\infty}=V_{\infty} / c
$$

For $M_{\infty} \geq 1$, bow shocks form ahead of the vehicle and radiate obliquely from the sides of a vehicle's leading edges, Figure 3.2. Pressure disturbances which effectively allow the downstream conditions to communicate with the upstream conditions are no longer able to propagate against the flow, leading to discontinuities (jumps) in some of the flow properties immediately upstream and downstream of the shock line.

In the following analysis, upstream properties of the inviscid ${ }^{6}$ free-stream flow (that are unaware of the vehicle's approach) are denoted with the subscript " $\infty$ ", and downstream properties near the boundary layer edge are denoted with a subscript " $e$ ". Properties in the stagnation region (behind the bow shock) are designated with subscript "st". It is usual to

${ }^{6}$ Inviscid describes a flow in which friction (shear resistance) is negligible, i.e. frictionless flow. 
change the reference coordinate system and consider the vehicle to be stationary and impacted by a gas flow with a velocity equal to the vehicle velocity, Figure 3.2.

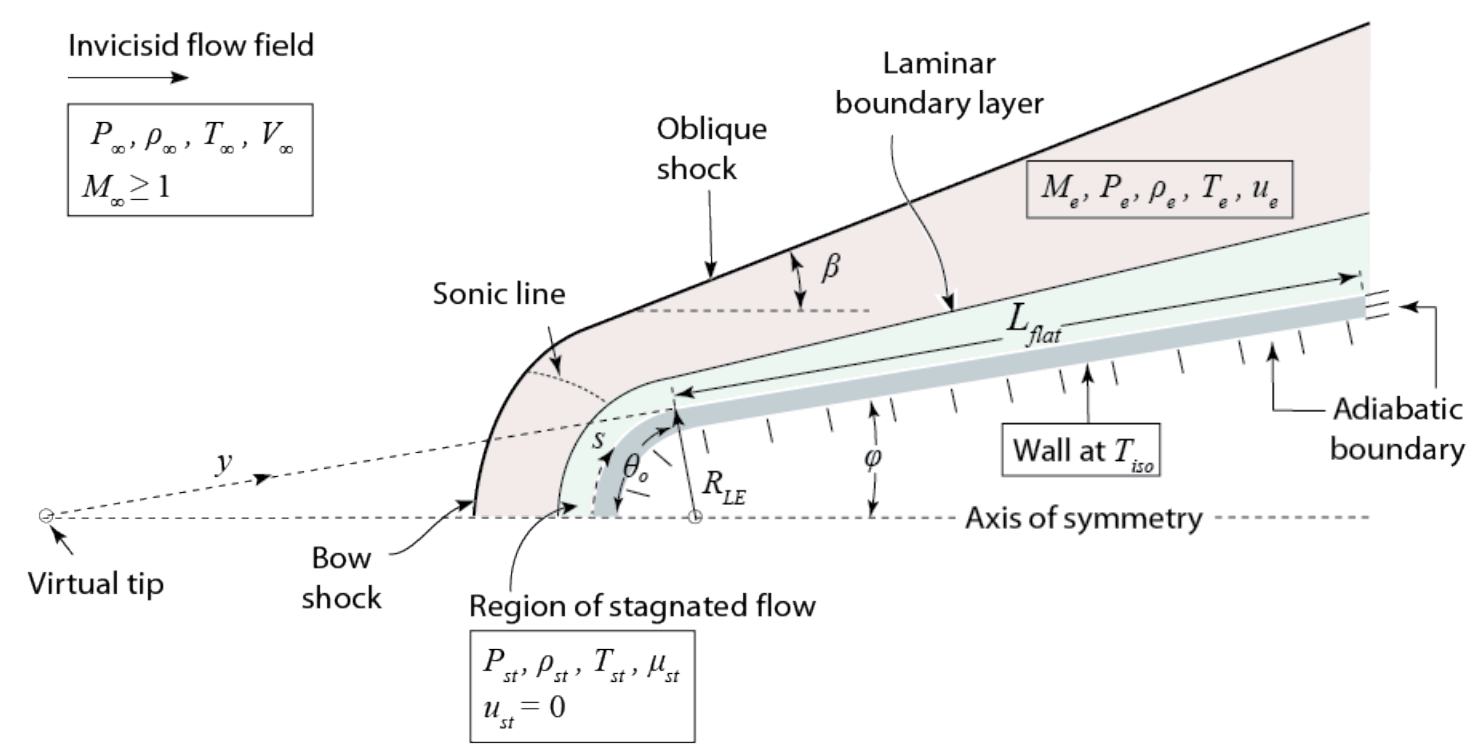

Figure 3.2. Shock pattern and flow variables around the leading edge. Adapted from [3].

To analyze the heat flux impinging on the vehicle as a function of the inviscid flow field properties, we make the following assumptions:

(i) The vehicle is undergoing steady, level flight;

(ii) No chemical or ionization reactions occur in the gas (see Chapter 2 for a discussion on the validity of this assumption); 
(iii) There is only weak viscous interaction between the boundary layer and inviscid flow, so that any influence in the inviscid flow results in negligible feedback on the boundary layer;

(iv) The boundary layer flow is laminar;

(v) A "no-slip" condition is imposed at the wall (i.e. all fluid velocity components are zero at the wall).

The leading edge system in Figure 3.2 has a leading edge radius $R_{L E}$ and wedge halfangle $\varphi$. The rear boundary is assumed adiabatic so that any heat which enters the leading edge at steady state is emitted from its surface (not the rear boundary). A design length $L_{\text {flat }}$ describes the length of the flat section that lies on a $y$-axis which extends from a virtual tip in front of the curved tip. A second, s-axis follows the tip curvature. The $y$-axis and s-axis are used for calculating the heat fluxes along the flat and curved sections, respectively. In the subsequent analysis, the leading edge radius $R_{L E}=3 \mathrm{~mm}$ and the wedge half-angle $\varphi=6^{\circ}$. These values are driven by aerodynamic design requirements, not thermal design requirements.

The thermal flux incident on the vehicle body depends on the atmospheric pressure $P_{\infty}$, density $\rho_{\infty}$, and temperature $T_{\infty}$, which vary with altitude. Thus, the combination of free-stream Mach number and altitude must be specified in the model order to compute a heating rate. The relationship between Mach number and altitude is established by the 
dynamic pressure at which the vehicle's engine was designed to operate [94]. For hydrocarbon-fueled scramjets, this is typically $48 \mathrm{kPa}$ [95]. Table 3.1 provides the relevant flight condition (Mach number and altitude) which meet this criterion, along with the associated thermodynamic properties for air at altitude.

The relevant temperature-dependent thermo-mechanical properties for the three alloys investigated - nickel-base IN718, niobium base C-103, and the TZM molybdenum alloy - are provided in Appendix A.

Table 3.1. Calculated free-stream and stagnation conditions for Mach 5-12 flight at a dynamic pressure $\left(\rho_{\infty} V_{\infty}^{2} / 2\right)$ of $48 k \mathrm{~Pa}[96]$.

\begin{tabular}{|c|c|c|c|c|c|c|c|}
\hline $\begin{array}{l}\text { Mach } \\
\text { No., } \\
M_{\infty}\end{array}$ & $\begin{array}{c}\text { Altitude } \\
(\mathrm{km})\end{array}$ & $\begin{array}{c}\text { Free-stream } \\
\text { temperature, } \\
T_{\infty}(\mathrm{K})\end{array}$ & $\begin{array}{c}\text { Free-stream } \\
\text { stagnation } \\
\text { temperature, } \\
T_{s t}(\mathrm{~K})\end{array}$ & $\begin{array}{c}\text { Free- } \\
\text { stream } \\
\text { static } \\
\text { pressure, } \\
P_{\infty}(\mathrm{Pa})\end{array}$ & $\begin{array}{c}\text { Free- } \\
\text { stream } \\
\text { density, } \\
\rho_{\infty} \\
\left(\mathrm{kg} / \mathrm{m}^{3}\right)\end{array}$ & $\begin{array}{c}\text { Free- } \\
\text { stream } \\
\text { velocity, } \\
V_{\infty} \\
(\mathrm{m} / \mathrm{s})\end{array}$ & $\begin{array}{c}\text { Adiabatic } \\
\text { wall } \\
\text { enthalpy, } \\
h_{s t, a w} \\
(\mathrm{MJ} / \mathrm{kg})\end{array}$ \\
\hline 5 & 24.5 & 221.08 & $1,326.46$ & $2,743.6$ & 0.0432 & 1,490 & 1.33 \\
\hline 6 & 26.9 & 223.44 & $1,832.18$ & $1,908.6$ & 0.0296 & 1,798 & 1.84 \\
\hline 7 & 29.0 & 225.48 & $2,435.13$ & $1,399.6$ & 0.0216 & 2,107 & 2.45 \\
\hline 8 & 30.8 & 227.24 & $3,135.97$ & $1,071.6$ & 0.0164 & 2,418 & 3.15 \\
\hline 9 & 32.3 & 229.12 & $3,940.81$ & 846.7 & 0.0129 & 2,731 & 3.96 \\
\hline 10 & 33.8 & 233.11 & $4,895.31$ & 685.7 & 0.0102 & 3,061 & 4.92 \\
\hline 12 & 36.3 & 240.18 & 7157.46 & 476.2 & 0.00691 & 3,728 & 7.19 \\
\hline
\end{tabular}




\subsection{Stagnation point heat flux}

The stagnation point is located within the boundary layer behind the bow shock and is defined as the region where the flow velocity in the s-axis direction, $u_{s t}$, is zero. The heat flux in the gas at the wall at the stagnation point of a slender body vehicle can be approximated from the two-dimensional flow past a cylindrical body [3];

$$
q_{s t, g w}=0.57 \operatorname{Pr}^{-0.6}\left(\rho_{s t} \mu_{s t}\right)^{1 / 2}\left(h_{s t, a w}-h_{w}\right) \sqrt{\frac{d u_{s t}}{d s}}
$$

where $\mathrm{Pr}$ is a non-dimensional Prandtl number ${ }^{7}$ and is taken as 0.715 for non-disassociating air [3]. The remaining fluid properties are the stagnation air density, $\rho_{s t}$, and viscosity, $\mu_{s t}$. The air density at the stagnation point $\rho_{s t}$ can be determined assuming it obeys the ideal gas law;

$$
\rho_{s t}=\frac{P_{s t}}{R T_{s t}}
$$

where $P_{s t}$ and $T_{s t}$ are the stagnation pressure and temperature behind the shock, and $R$ is the specific gas constant (for dry air, $R=287 \mathrm{~J} / \mathrm{kgK}$ ). The stagnation temperature does not change across a shock [3], so $T_{s t}$ is taken as the free stream stagnation temperature. $P_{s t}$

7 The Prandtl number is the ratio of a fluid's momentum diffusivity to its thermal diffusivity (a measure of its ability to transfer energy by convection versus conduction) 
is found from the normal shock relations for a perfect gas which relate the upstream and downstream properties across a shock to the Mach number and heat capacity ratio, $\gamma[3]$;

$$
P_{s t}=P_{\infty}\left[\frac{(\gamma+1) M_{\infty}^{2}}{2+(\gamma-1) M_{\infty}^{2}}\right]^{\gamma / \gamma-1}\left[\frac{(\gamma+1)}{2 \gamma M_{\infty}^{2}-(\gamma-1)}\right]^{1 / \gamma-1}
$$

where $P_{\infty}$ is the free-stream pressure. For non-catalytic air flow, $\gamma=1.4$ [3]. The viscosity term, $\mu_{s t}$, of equation (3.4) can be estimated using either the viscosity power law or Sutherland equation [97], both of which relate fluid viscosity to temperature. The latter is used here [97];

$$
\frac{\mu}{\mu_{\text {Ref }}}=\left(\frac{T_{\text {Ref }}+C}{T+C}\right)\left(\frac{T}{T_{\text {Ref }}}\right)^{3 / 2}
$$

where $C=120 \mathrm{~K}$ for air and reference parameters $\mu_{R e f}=1.827 \times 10^{-5} \mathrm{kgm}^{-1} \mathrm{~s}^{-1}$ at $T_{\text {Ref }}=291.15 \mathrm{~K}[97]$.

Equation (3.4 states that the heat flux through the boundary layer to the vehicle surface is driven by the difference in the adiabatic wall enthalpy and the actual wall enthalpy, $\left(h_{a w}-h_{w}\right)$. These wall enthalpies are given by;

$$
\begin{gathered}
h_{a w}=C_{p, a w} T_{a w} \\
h_{w}=C_{p, w} T_{i s o}
\end{gathered}
$$


where $C_{p, a w}$ and $C_{p, w}$ are the temperature-dependent specific heats of the wall material operating at wall temperatures $T_{a w}$ and $T_{i s o}$, respectively. A wall at $T_{a w}$ balances the impinging heat flux with radiation cooling so there is no net heat transfer. $T_{a w}$ is, of course, not known. However, under an adiabatic condition, we can equate the enthalpy of the flow to the enthalpy of the wall in order to pose equation (3.8) in terms of known flow variables [3];

$$
h_{a w}=h_{\infty}=C_{p \infty} T_{\infty}+\frac{V_{\infty}^{2}}{2}
$$

where $C_{p \infty}$ is the specific heat of air (taken as $C_{p \infty}=1006 \mathrm{~J} / \mathrm{kgK}$ ). $T_{\infty}$ and $V_{\infty}$ are the free-stream static temperature and velocity, respectively. Values for the adiabatic wall enthalpy are provided for various flow conditions in Table 3.1.

The $\frac{d u_{s t}}{d s}$ term in equation $(3.4$ describes the rate at which the velocity at the outer edge of the boundary layer increases along the surface curvature (s-axis) from the stagnation point. This is given by [3];

$$
\frac{d u_{s t}}{d s}=\frac{1}{R_{L E}} \sqrt{\frac{2\left(P_{s t}-P_{\infty}\right)}{\rho_{s t}}}
$$

Substituting equation (3.11) for $\frac{d u_{s t}}{d s}$ in equation (3.4), reveals that the heat flux is inversely proportional to the square root of the leading edge radius, $R_{L E}$ : an aerodynamic design requiring sharp leading edges increases the incident heat by the factor $R_{L E}{ }^{-1 / 2}$. 


\subsection{Heat flux around the tip curvature}

The flow rapidly accelerates away from the stagnation point around the leading edge tip curvature and becomes entrained in the free-stream flow. The increase in velocity requires a transfer of potential and internal energy to kinetic energy, decreasing the available heat for transfer to the vehicle. As a result, the incident heat transfer falls over the curved portion of the tip as the flow accelerates. Lees [98] derived an expression for the heat flux incident upon a hemispherical surface, $q_{t i p, g w}$, as a function of the stagnation point heat flux;

$$
\frac{q_{t i p, g w}}{q_{s t, g w}}=2 \theta \sin \theta\left[\left(1-\frac{1}{\gamma M_{\infty}^{2}}\right) \cos ^{2} \theta+\frac{1}{\gamma M_{\infty}^{2}}\right] G^{-1 / 2}
$$

where

$$
\begin{array}{r}
G=\left(1-\frac{1}{\gamma M_{\infty}^{2}}\right)\left[\theta^{2}-\frac{\theta}{2} \sin 4 \theta+\frac{(1-\cos 4 \theta)}{8}\right] \\
+\frac{4}{\gamma M_{\infty}^{2}}\left[\theta^{2}-\theta \sin 2 \theta+\frac{(1-\cos 2 \theta)}{2}\right]
\end{array}
$$

$\theta$ is the angle between the flight direction and radius vector from the center of curvature of the nose (c.f. Figure 3.2) and is given by $\theta=s / R_{L E}$. As $s$ approaches $0, \frac{q_{t i p, g w}}{q_{s t, g w}} \rightarrow 1$. 


\subsection{Heat flux over the flat surface}

The heat flux of the boundary gas layer that impinges on the flat portion of the vehicle, $q_{f, g w}$, can be evaluated by analyzing the simple case of a flat plate in a high enthalpy flow [99];

$$
q_{f, g w}=C_{H} \rho_{e} u_{e}\left(h_{e, a w}-h_{w}\right)
$$

where $C_{H}$ is the dimensionless Stanton number (the ratio of heat transferred into a fluid to the fluid's heat capacity). Equation (3.14) states that the heat flow through the boundary layer gas is driven by the difference in enthalpies. The wall enthalpy $h_{w}$ is given by equation (3.9). The adiabatic wall enthalpy is [99];

$$
h_{e, a w}=C_{p \infty} T_{e}+r \frac{u_{e}^{2}}{2}
$$

where $r$ is a recovery factor. Its introduction permits the application of simple, low speed, inviscid flow equations to high speed, viscous flow by accounting for dissipation effects. For laminar flow, $r \approx \sqrt{\operatorname{Pr}}$ [97]. The flow properties near the edge of the boundary layer, namely $\rho_{e}, u_{e}$, and $T_{e}$ (density, velocity, and temperature behind the oblique shock) are given by the oblique shock relations, which relate these properties to those of the free stream [3], [8], [100];

$$
\rho_{e}=\rho_{\infty} \frac{(\gamma+1) M_{\infty}^{2} \sin ^{2} \beta}{2+(\gamma-1) M_{\infty}^{2} \sin ^{2} \beta}
$$




$$
\begin{gathered}
u_{e}=u_{\infty} \frac{M_{e}}{M_{\infty}}\left(\frac{P_{e} \rho_{\infty}}{P_{\infty} \rho_{e}}\right)^{1 / 2} \\
T_{e}=\frac{P_{e} \rho_{\infty}}{P_{\infty} \rho_{e}} T_{\infty}
\end{gathered}
$$

where $\beta$ is the oblique shock wave angle which can be found from;

$$
\begin{aligned}
\operatorname{Tan} \beta= & {\left[\left(\frac{\gamma+1}{2}\right)\left(\frac{M_{\infty}^{2}}{M_{\infty}^{2} \sin ^{2} \beta-1}\right)-1\right]^{-1} \operatorname{Cot} \varphi } \\
M_{e}= & {\left[\frac{1}{\sin ^{2}(\beta-\varphi)} \cdot \frac{M_{\infty}^{2} \sin ^{2} \beta+\frac{2}{\gamma-1}}{\frac{2 \gamma M_{\infty}^{2} \sin ^{2} \beta}{\gamma-1}-1}\right]^{1 / 2} } \\
& \frac{P_{e}}{P_{\infty}}=1+\frac{2 \gamma}{1+\gamma}\left(M_{\infty}^{2} \sin ^{2} \beta-1\right)
\end{aligned}
$$

Equation (3.19) can be iteratively solved for the shock wave angle $\beta$ (or a lookup table can be used, see [100]).

To determine the Stanton number, we employ the reference temperature concept which adapts incompressible flow formulas to compressible flows by replacing thermodynamic and transport properties for incompressible flow with those evaluated at a reference temperature, $T^{*}$. The correlation used here is given by Eckert [97];

$$
\frac{T^{*}}{T_{e}}=0.5+0.039 M_{e}^{2}+0.5 \frac{T_{i s o}}{T_{e}}
$$


Using the above empirical correlation for $T^{*} / T_{e}$, a reference friction factor, $C_{f}^{*}$, can be computed from [97];

$$
C_{f}^{*}=0.664\left(\frac{T^{*}}{T_{e}}\right)^{-1 / 6} \operatorname{Re}_{y}^{-1 / 2}
$$

where the Reynolds number is a function of distance $y$ taken from a virtual tip (c.f. Figure $3.2)$;

$$
\operatorname{Re}_{y}=\frac{\rho_{e} u_{e} y}{\mu_{e}}
$$

As before, the viscosity can be determined from the Sutherland relation (equation (3.7)). A reference Prandtl number can be approximated as [97];

$$
\operatorname{Pr}^{*} \approx\left[\left(\frac{T_{a w}}{T_{e}}-1\right)\left(\frac{2}{(\gamma-1) M_{e}^{2}}\right)\right]^{2}
$$

The reference friction factor and Prandtl number can then be used to determine the Stanton number using the Reynolds analogy [97];

$$
C_{H}=\frac{C_{f}^{*}}{2 \operatorname{Pr} * 2 / 3}
$$




\subsection{Solution of the isothermal wall temperature}

The wall temperature in the isothermal limit can be solved using a cumulative, steady state heat balance over the vehicle surface which is expressed as a summation of integrals [94];

$$
\int_{0}^{R_{L E} \theta_{0}}\left(q_{s t, g w} \frac{q_{t i p}}{q_{s t, g w}}+q_{r}\right) d s+\int_{R_{L E} \tan \theta_{0}}^{L_{f l a t}+R_{L E} \tan \theta_{0}}\left(q_{f, g w}+q_{r}\right) d y=0
$$

where the heat flux radiated away from the vehicle surface is;

$$
q_{r}=-\sigma \varepsilon T_{i s o}^{4}
$$

The Stefan-Boltzmann constant $\sigma=5.67 \times 10^{-8} \mathrm{~W} / \mathrm{m}^{2} \mathrm{~K}^{4}$ and $\varepsilon$ is the emissivity (set to 0.95 here). In the integral bounds, $R_{L E} \tan \theta_{0}$ is the distance from the virtual tip to the start of the flat surface and $\theta_{o}=\frac{\pi}{2}-\varphi$.

Because the specific heat capacity of the wall is temperature-dependent, a wall temperature must be guessed by assuming an initial value for the heat capacity, and equation (3.27) numerically solved for $T_{i s o}$. The new wall temperature is then used to recalculate the specific heat capacity and the heat balance equation is again solved. The solution of the wall temperature quickly converges upon several iterations. In this work, the false position method [101] is used to converge within $0.1 \%$ of the exact solution. 
Figure 3.3 shows the incident heat flux for an IN718 leading edge at several Mach numbers. For a fixed Mach number flow, the incident flux is highest at the tip and falls rapidly as the curved tip region transitions to the planar part of the wedge and then falls slowly as the adiabatic edge is approached. The maximum incident flux rises from 0.1 to $1.0 \mathrm{MWm}^{-2}$ as the flow velocity increases from $M_{\infty}=2$ to $M_{\infty}=6$ (i.e. as one approaches the hypersonic regime). The values for $T_{i s o}$ listed on the plot are the solved isothermal equilibrium temperatures of the wedge. It should be noted that the only material property which factors into the calculation is the specific heat capacity of the alloy.

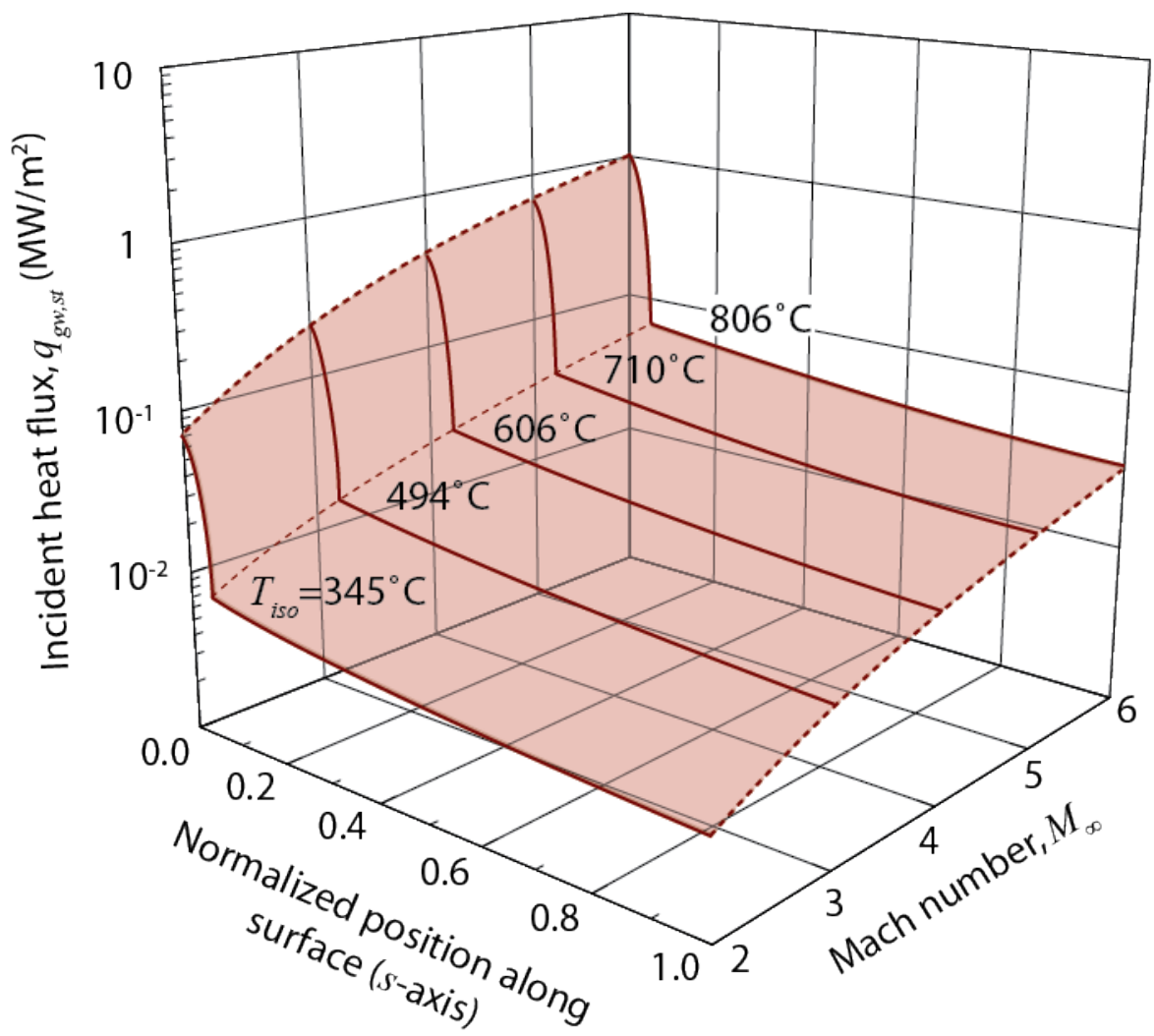

Figure 3.3. The heat flux incident upon an IN718 leading edge for $M_{\infty}=2$ to 6 at the solved isothermal wall temperatures. $R_{L E}=3 \mathrm{~mm}, \varphi=6^{\circ}, L_{\text {flat }}=82.3 \mathrm{~mm}$. 
Figure 3.4 plots the predicted wall temperature as a function of design length, $L_{\text {flat }}$, for several Mach numbers and each of the three materials systems. Increases in design length provide a larger radiating surface for cooling, thereby lowering wall temperatures. The plots of C-103 and TZM are nearly identical, since these have similar specific heat capacities. However, IN718 has over twice the specific heat capacity at high temperatures and, as a result, wall temperatures are lower. The respective melting temperatures are noted in the plots which guide selection of an appropriate design length. The melting temperature of IN718 will not be exceeded for flight velocities of Mach 6 or lower, but care must be taken to choose a design length which is large enough for higher flight speeds. For C-103 and TZM, flight speeds as high as Mach 12 may be accessible with reasonable lengths. It is with uncertainty that this is stated this since, at this point in the analysis, elevated temperatures at the tip have yet to be considered. The maximum temperature is an important design consideration during alloy selection since it will dictate the temperature-dependent material strength and thermo-chemical (oxidation) resistance of the wall alloy. Its solution is presented in the next section. 

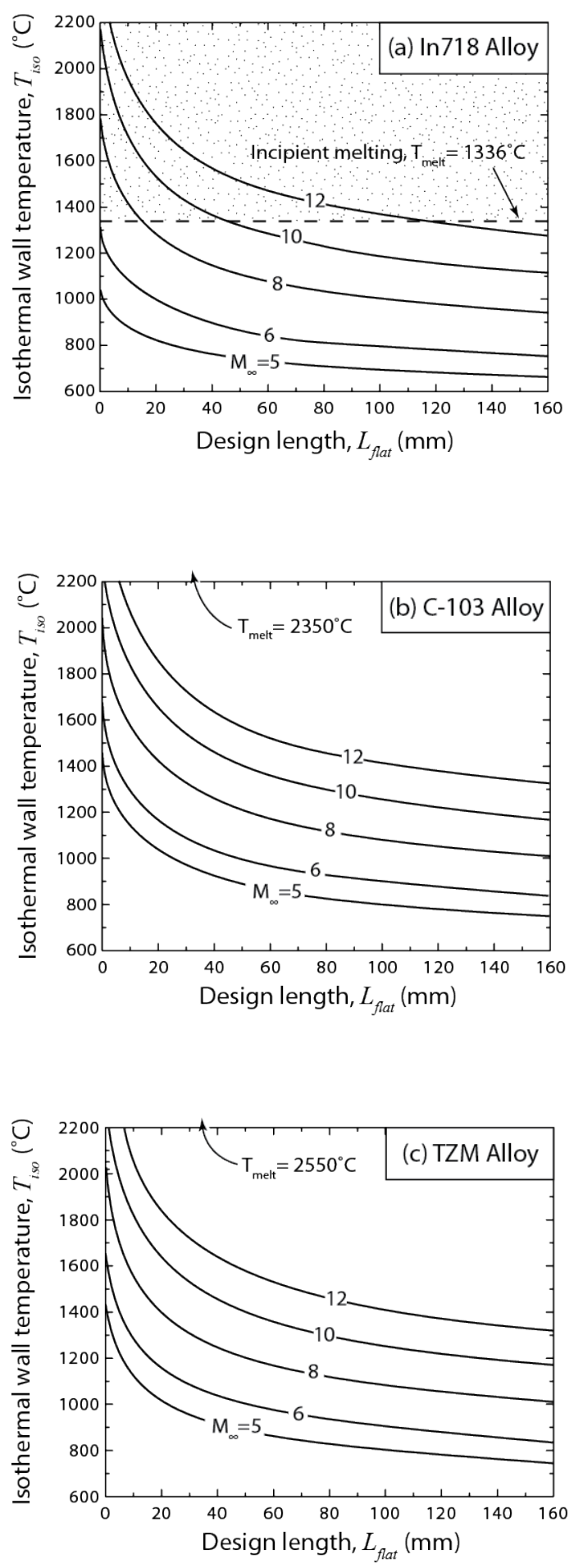

Figure 3.4. Isothermal wall temperatures for (a) IN718, (b) C-103, and (c) TZM alloys as a function of length for free-stream Mach numbers 5-12. $R_{L E}=3 \mathrm{~mm}$ and $\varphi=6^{\circ}$. 


\section{Solution of the maximum temperature and thermal stress}

The assumption of an isothermal wall temperature is defensible through the examination of experimental heat pipe test results from traditional cylindrical structures [72], [73], [74] and flat vapor chamber designs [102]. However, the large heat flux gradient, shown in Figure 3.3, gives rise to a localized but steep temperature drop in the wall's through-thickness direction at the tip. Because a heat pipe operates by redistributing its heat internally (i.e. through the vapor core of the sealed system), a large amount of heat, being driven by the thermal gradient, is conducted through the tip's wall. A larger gradient transports more heat but also induces greater thermal stresses which are created when the thermal expansion of the hot solid is confined by cooler surrounding wall material.

Consider two strips of material of identical chemical composition, temperature, and size. Should one now be heated, as drawn in Figure 3.5(a), its expansion gives rise to a thermal strain, $\alpha \Delta T$, where $\alpha$ is the material's thermal expansion coefficient. Now consider the case of the leading edge wall, shown in (b). The hot stagnation gases give rise to temperature $T_{\text {max }}$ at the tip's surface, while the inside wall temperature (at the wall-wick interface) is $\approx T_{i s o}$. The magnitude of the temperature difference, $\Delta T=T_{\text {max }}-T_{\text {iso }}$, can be quite large for hypersonic enthalpies. Unlike the unconstrained situation, a strain misfit 
between the warmer and cooler material gives rise to a compressive thermal stress, $\sigma_{t h}$. The maximum tip stresses which are produced can be determined from [14];

$$
\sigma_{t h}=\frac{E \alpha\left(T_{\max }-T_{i s o}\right)}{1-\nu}
$$

where $E, \alpha$, and $\nu$ are the Young's modulus, the thermal expansion coefficient, and Poisson's ratio, respectively, of the case alloy. Values for the three alloys can be found in Appendix A. Alloys which are stiff at the use temperature (high $E$ ) and have a large $\alpha$ value produce the highest thermal stresses.. Face sheet wrinkling (by instantaneous plastic deformation) occurs if this stress exceeds the material's yield strength $\left(\sigma_{y}>\sigma_{t h}\right)$ using the Tresca yield criterion. Finite element simulations on leading edge heat pipes by Steeves et. al. [94] showed good agreement with the stress model presented here. 
(a) Unconstrained material

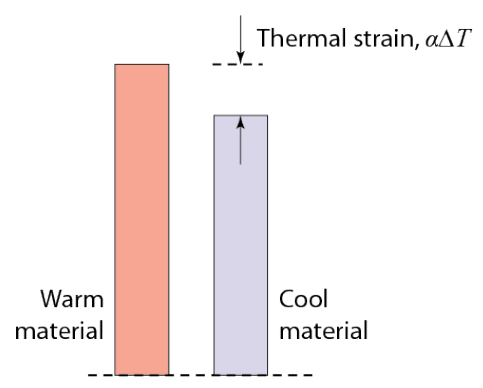

(b) Constrained thermal expansion

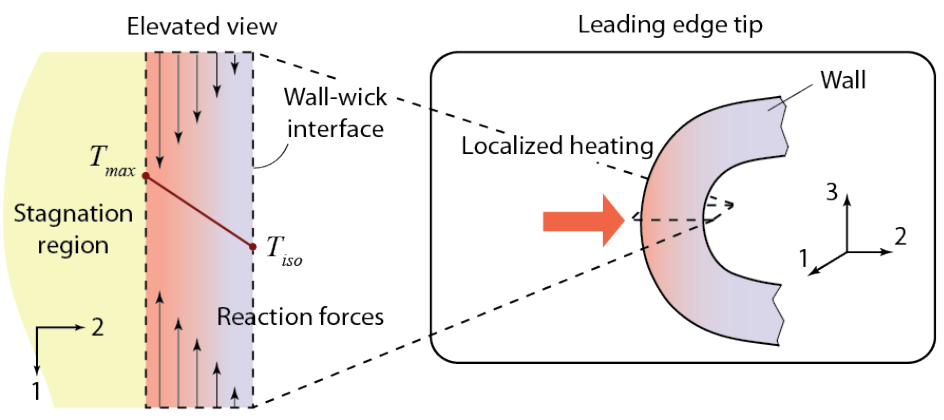

Figure 3.5. Schematic illustration of thermal stress state at the leading edge tip.

(a) Unconstrained material showing strain misfit due to differential thermal expansion.

(b) Localized heating generates a through-thickness stress gradient which causes failure when the yield strength is exceeded.

To determine the maximum stresses, the maximum wall temperature $T_{\text {max }}$ must be determined. Figure 3.6 shows an enlarged view of the leading edge tip with a case wall thickness of $b_{\text {wall }}$. $T_{\text {max }}$ is found on the external surface at the stagnation point. If the fluid in the wick is near the working fluid's liquid-vapor saturation temperature, then the internal wall surface can be approximated by the isothermal temperature $T_{i s o}$. 


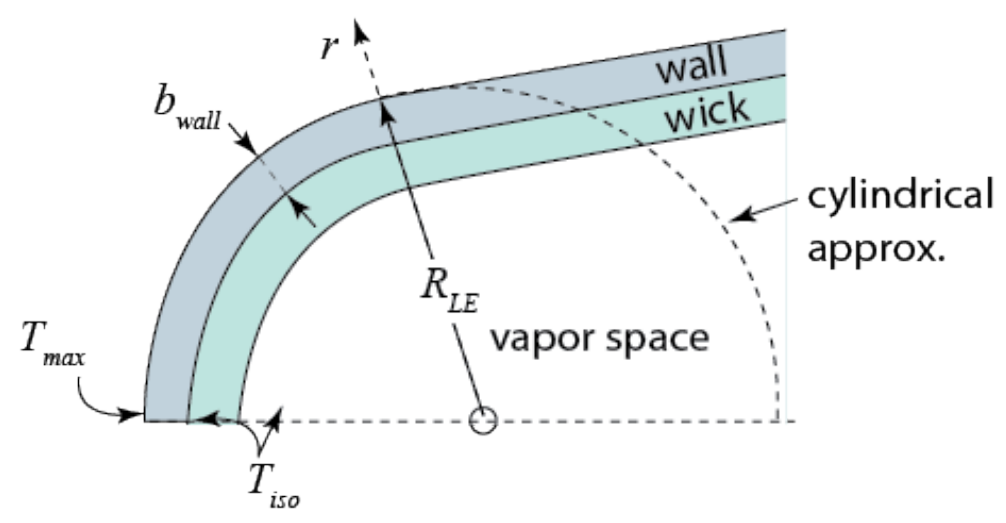

Figure 3.6. Enlarged view of the leading edge.

The wall heat flux at the tip can be found from the steady state heat equation for a hollow cylinder. In cylindrical coordinates this takes the form [103];

$$
\frac{d}{d r}\left(r \frac{d T}{d r}\right)=0
$$

where $r$ is the radius to the outer surface of the wall. The boundary conditions are;

$$
\begin{gathered}
T\left(R_{L E}-b_{\text {wall }}\right)=T_{\text {iso }} \\
T\left(R_{L E}\right)=T_{\max }
\end{gathered}
$$

Equation (3.30) can be solved to yield an expression for the temperature distribution through the wall; 


$$
T(r)=T_{\text {iso }}+\left(T_{\text {iso }}-T_{\text {max }}\right) \frac{\ln \left(\frac{r}{R_{L E}-b_{\text {wall }}}\right)}{\ln \left(\frac{R_{L E}}{R_{L E}-b_{\text {wall }}}\right)}
$$

Differentiation in the radial coordinate gives;

$$
\frac{d T}{d r}=\frac{\left(T_{i s o}-T_{\max }\right)}{r \ln \left(\frac{R_{L E}}{R_{L E}-b_{w a l l}}\right)}
$$

Fourier's law of conduction states the conducted heat flux;

$$
q=-k \frac{d T}{d r}
$$

Upon substituting (3.34) for $d T / d r$, the heat flux through the external surface evaluated at $r=R_{L E}$ is;

$$
q_{s t, w}=\frac{k_{\text {wall }}\left(T_{\text {max }}-T_{\text {iso }}\right)}{R_{L E} \ln \left(\frac{R_{L E}}{R_{L E}-b_{\text {wall }}}\right)}
$$

where $k_{\text {wall }}$ is the wall's thermal conductivity. An energy balance at the stagnation point can be written;

$$
q_{s t, w}=q_{s t, g w}+q_{s t, r}
$$

where $q_{s t, g w}$ is solved according to the procedure outlined in the previous section, and where radiative heat flow away from the vehicle at the tip is approximated by; 


$$
q_{s t, r}=-\sigma \varepsilon T_{\max }^{4}
$$

Combining equations (3.36), (3.37), and (3.38) gives an expression for the maximum temperature at the tip;

$$
T_{\text {max }}=\frac{R_{L E}}{k_{\text {wall }}} \ln \left(\frac{R_{L E}}{R_{L E}-b_{\text {wall }}}\right)\left(q_{s t, g w}\left(T_{i s o}\right)-\sigma \varepsilon T_{\text {max }}^{4}\right)+T_{i s o}
$$

Equation (3.39) can be iteratively solved for $T_{\max }$ for a chosen combination of $L_{\text {flat }}$ and $b_{\text {wall }}$. Figure 3.7, Figure 3.8, and Figure 3.9 show the variation of the wall thickness with design length for constant contours of $T_{\max }$ for IN718 alloy, C-103 alloy, and the MoTZM alloy, respectively. The general trend shows that increasing the design length lowers the maximum wall temperature. This is because a larger radiating surface (i.e. cooling) lowers $T_{i s o}$ and since the tip's wall conduction is proportional to $\left(T_{\max }-T_{\text {iso }}\right), T_{\max }$ also decreases to balance the fluxes at the tip. At longer design lengths, the maximum temperature becomes less sensitive to changes in length and at shorter design lengths, the maximum temperatures becomes less sensitive to changes in wall thickness. 

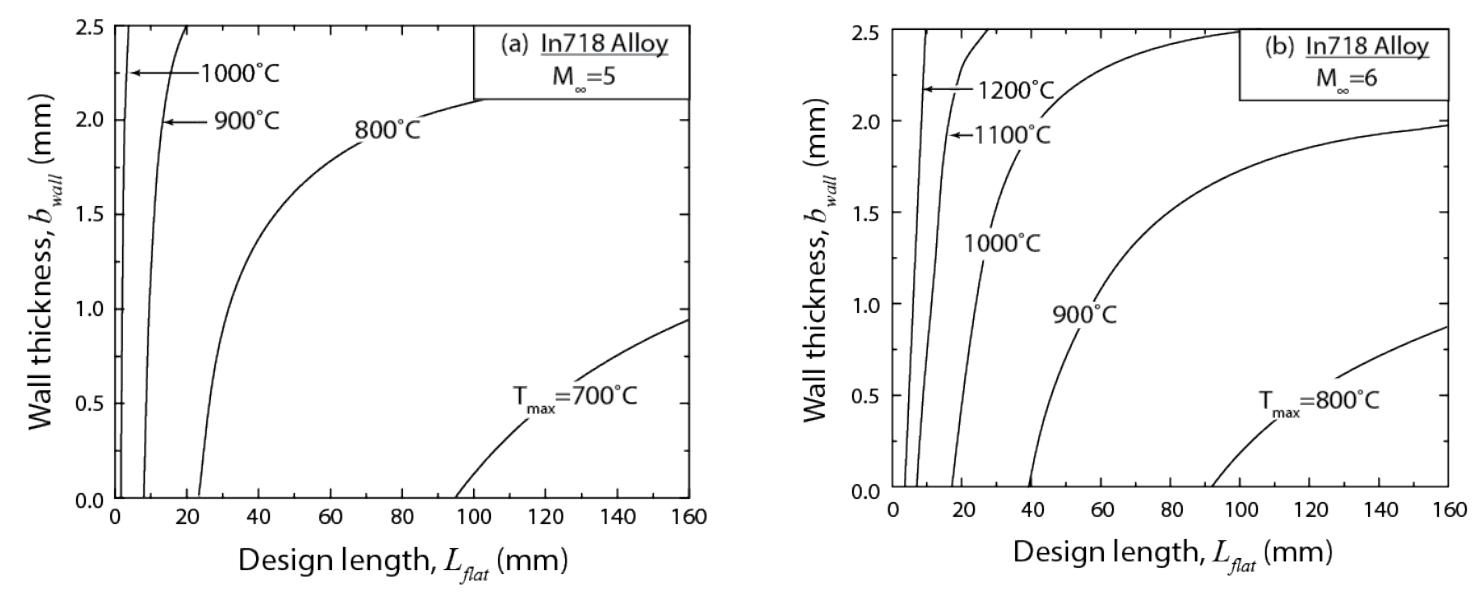

Figure 3.7. Effects of the wall thickness and design length upon $T_{\text {max }}$ for IN718 alloy leading edge in (a) Mach 5 (24.5km) and (b) Mach 6 (26.9km) flows. $R_{L E}=3 \mathrm{~mm}$ and $\varphi=6^{\circ}$
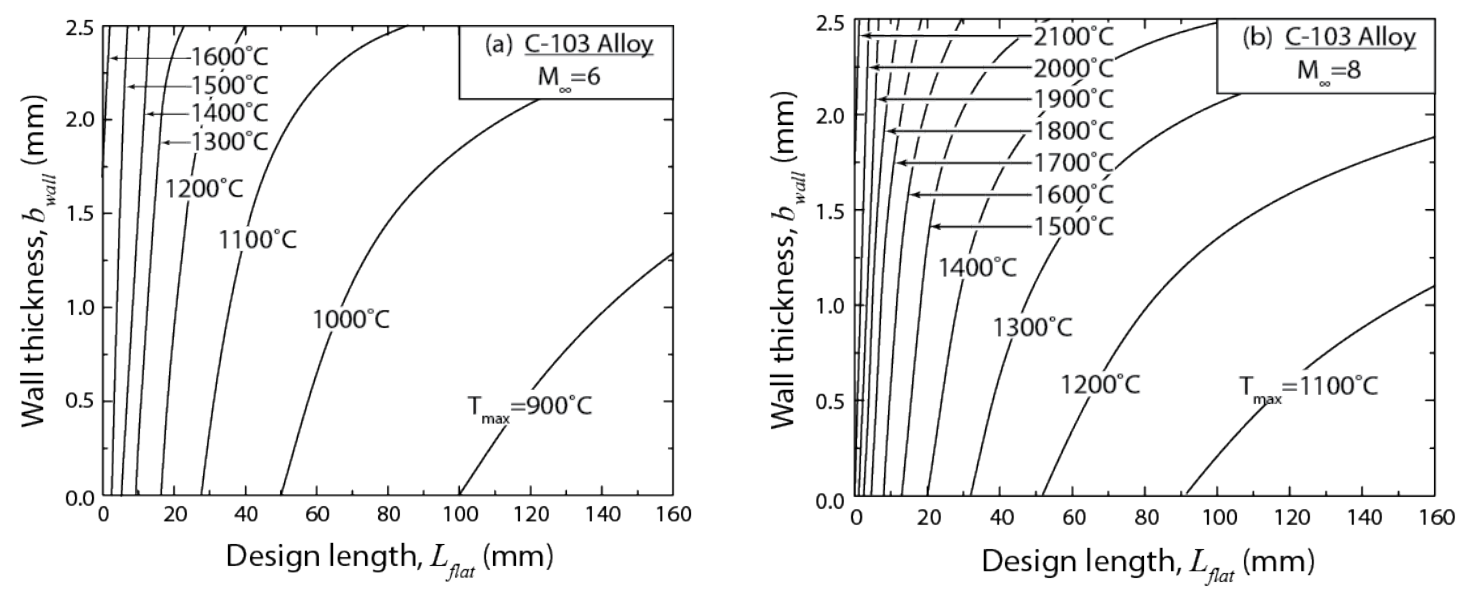

Figure 3.8. Effects of the wall thickness and design length upon $T_{\text {max }}$ for $C-103$ alloy leading edge in (a) Mach 6 (26.9km) and (b) Mach 8 (30.8km) flows. $R_{L E}=3 \mathrm{~mm}$ and $\varphi=6^{\circ}$ 

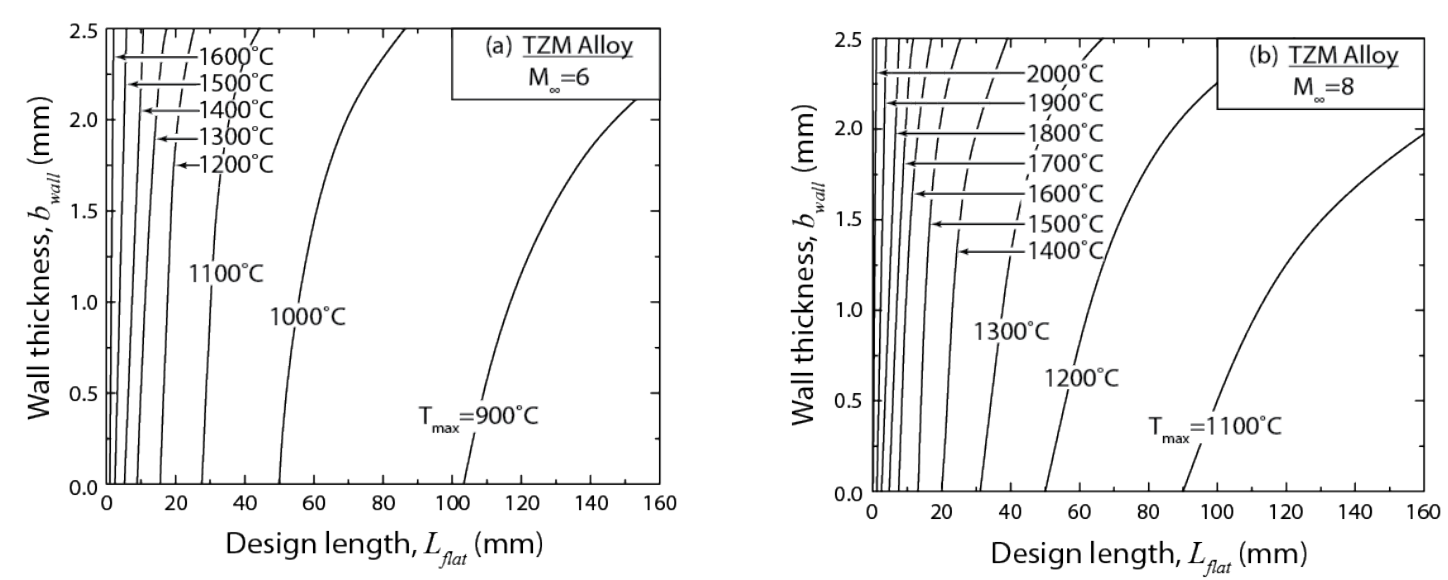

Figure 3.9. Effects of the wall thickness and design length upon $T_{\text {max }}$ for TZM alloy leading edge in (a) Mach 6 (26.9km) and (b) Mach 8 (30.8km) flows. $R_{L E}=3 \mathrm{~mm}$ and $\varphi=6^{\circ}$

The specific heat capacity and thermal conductivity are the only wall material properties which influence the maximum temperature. A comparison of IN718 alloy and C-103 alloy operating at Mach 6 (Figure 3.7(b) and Figure 3.8(a), respectively) shows that IN718 alloy operates at a lower $T_{\max }$ due to its higher specific heat capacity. A similar comparison between C-103 alloy and TZM alloy shows that TZM alloy operates at a lower $T_{\text {max }}$, yet these alloy heat capacities are nearly identical; in this case, the lower maximum temperature comes from TZM's higher thermal conductivity at elevated temperatures. 


\subsection{Design maps}

The above equations and analysis can be used to generate a single plot from which all relevant geometric design parameters $\left(L_{\text {flat }}, b_{\text {wall }}\right)$ and operating conditions $\left(T_{w}, T_{\text {max }}, \sigma_{t h}\right)$ can be read. Design maps for an IN718 alloy leading edge heat spreader operating at Mach 5 and 6 are shown in Figure 3.10. Figure 3.11 and Figure 3.12 provide maps for the C-103 and TZM alloys, respectively, operating at Mach 6 and 8. The maps show criss-crossing contours of design parameters $L_{\text {flat }}$ and $b_{\text {wall }}$ mapped onto a space of operating conditions $T_{\text {max }}$ and $\sigma_{t h}$. To use the map, a value for $L_{f l a t}$ and $b_{\text {wall }}$ are chosen and their intersection is located. The value for $T_{\max }$ and $\sigma_{t h}$ can then be read from the abscissa and ordinate axes. The isothermal wall temperature is found by following the $L_{\text {flat }}$ contour to the abscissa axis $\left(b_{\text {wall }} \rightarrow 0\right)$ and reading the intersecting temperature.

The unshaded region forms the permissible design space and values for $L_{\text {flat }}$ and $b_{\text {wall }}$ should be chosen from this space. It is bounded to the upper left by a maximum value for $L_{\text {flat }}$. Design lengths beyond those plotted result in turbulent flow and the hypersonic flow theory upon which this analysis is based becomes invalid. To the upper right, the bound is formed by the alloy's yield strength also plotted in the figures. The one exception is TZM under Mach 6 conditions, whose upper right bound is formed by the $b_{\text {wall }}=3 \mathrm{~mm}$ contour, indicating that there is no design condition for which the wall material will plastically deform. 
The plots indicate that as Mach number increases, the available design space decreases due to the more demanding thermal environment. Increases to $b_{\text {wall }}$ (which increases $T_{\text {max }}$ ) and $L_{\text {flat }}$ (which lowers $T_{\text {iso }}$ ) raise the thermal stress at the tip (which is proportional to $\left.T_{\max }-T_{i s o}\right)$. A high alloy thermal conductivity will also expand the design space by lowering the temperature differential between the maximum and isothermal temperatures, increasing the slope of the $L_{\text {flat }}$ contours. A high elastic modulus and high thermal expansion coefficient increase thermal stresses and push the $b_{\text {wall }}$ contours upwards, squeezing the design space against the yield curve. Figure 3.10 shows that IN718 can be used at both Mach 5 and Mach 6, so long as the design length is kept shorter than $180 \mathrm{~mm}$ and the wall thickness is kept sufficiently thin (no greater than $1.6 \mathrm{~mm}$ for Mach 6 over most design lengths). Figure 3.11 shows that C-103 can perform at Mach 8 with a design length of up to $240 \mathrm{~mm}$ so long as the wall thickness is less than $1.5 \mathrm{~mm}$. Under this condition, the maximum temperature is less than $1100^{\circ} \mathrm{C}$ and the isothermal wall temperature falls well below $1000^{\circ} \mathrm{C}$. TZM alloy (Figure 3.12) has the largest available design space, owing to its good combination of high thermal conductivity and high yield strength at elevated temperatures. 

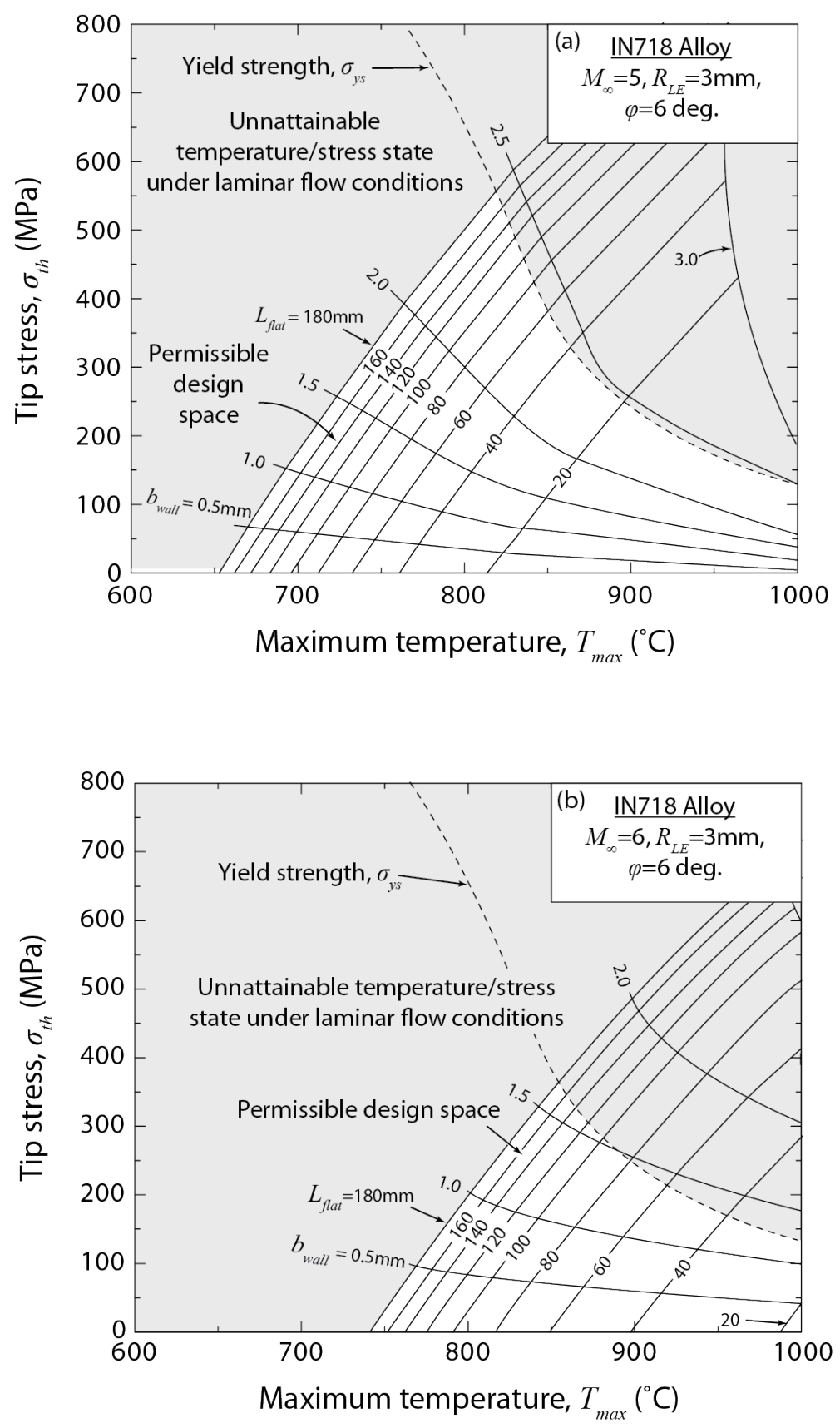

Figure 3.10. IN718 alloy design maps for a leading edge operating at (a) Mach 5 and (b) Mach 6. 

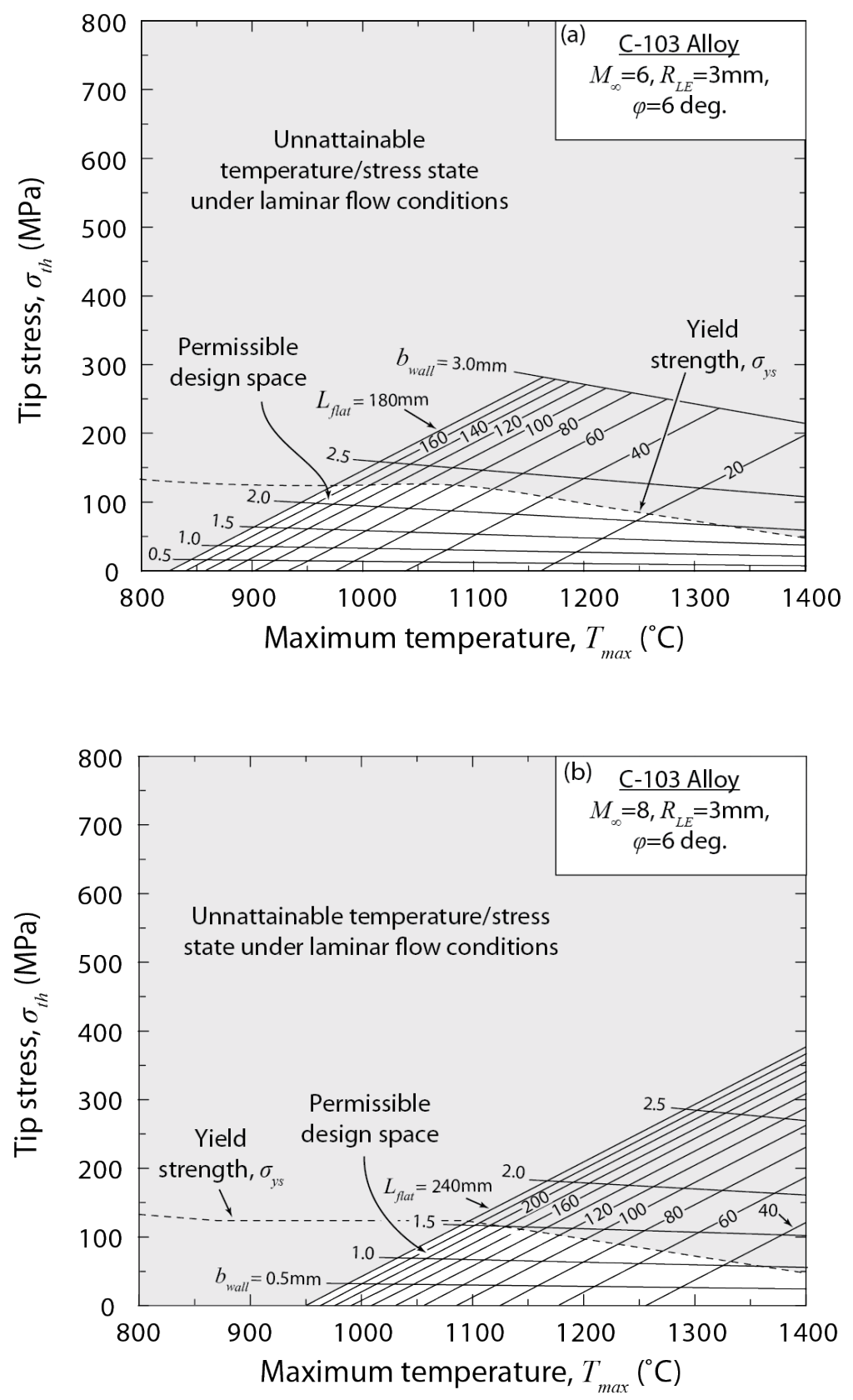

Figure 3.11. C-103 alloy design maps for a leading edge operating at (a) Mach 6 and (b) Mach 8. 

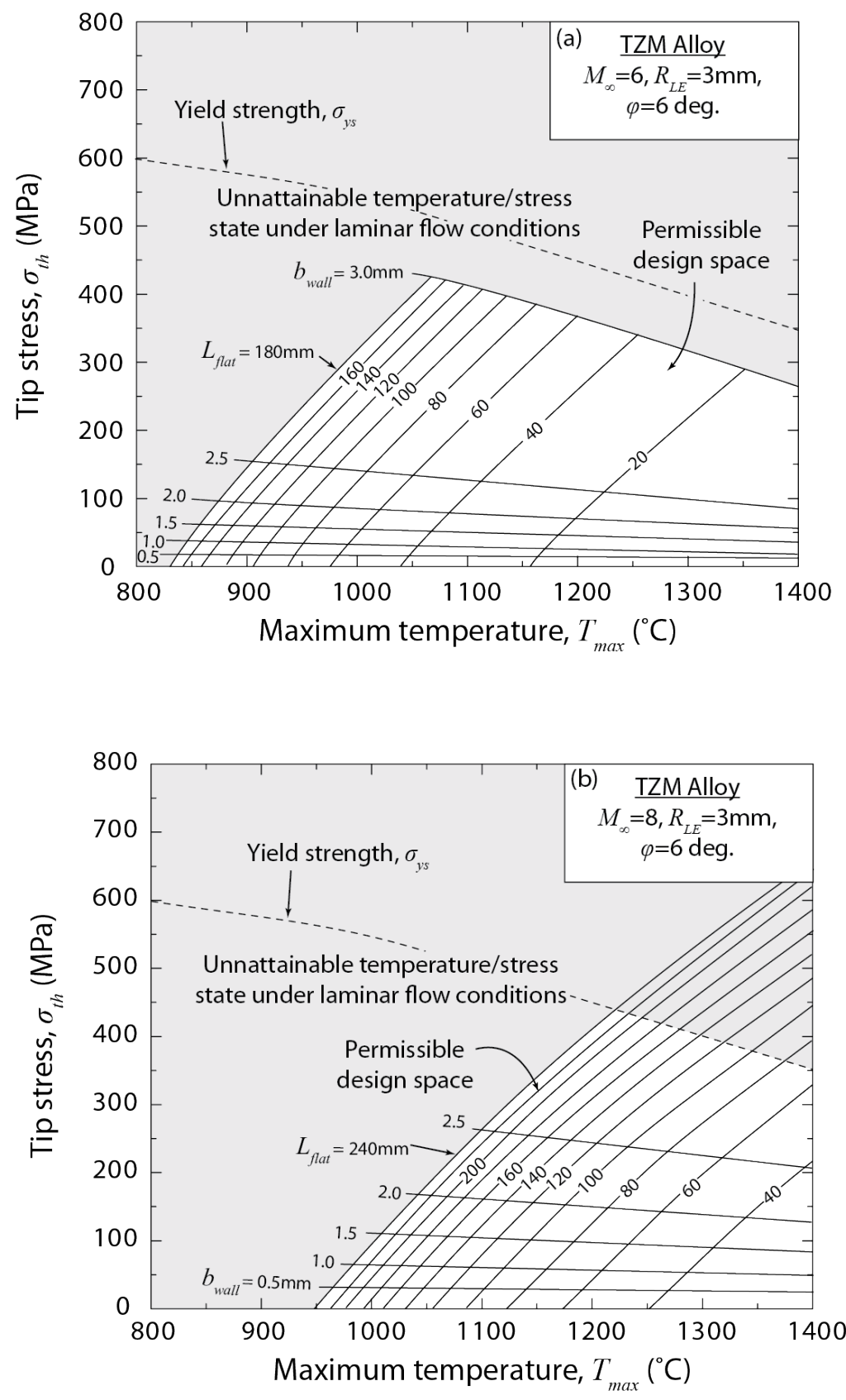

Figure 3.12. TZM alloy design maps for a leading edge operating at (a) Mach 6 and (b) Mach 8. 


\subsection{Summary}

A methodology for the design of a leading edge heat spreader has been presented which examines the influence of design parameters (overall length, wall thickness, and alloy) on operating conditions (isothermal temperature, maximum temperature, thermal stress). The methodology employs a model which solves for an isothermal operating temperature using a cumulative heat flux balance obtained from hypersonic flow equations. A maximum temperature is then obtained using a local energy balance at the tip and the thermally induced stresses are determined. Parameterization of the design variables allows the formulation of maps which show the feasibility of designs for a hypersonic flight condition. Three candidate systems for heat pipe leading edges have been explored; IN718, C-103, and TZM. Provided the right combination of design length and wall thickness are selected, IN718 are candidates for Mach $5(24.5 \mathrm{~km})$ and Mach $6(26.9 \mathrm{~km}) \mathrm{flight}$, and C-103 and TZM for Mach $6(26.9 \mathrm{~km})$ and Mach $8(30.8 \mathrm{~km})$ flight.

The design methodology presented in this chapter treats the internal heat spreading operation of the heat pipe as a "black box". The assumption is that the heat is so effectively spread throughout the vapor space that the system is isothermalized (except at the tip). It is therefore necessary to extend the methodology to account for potential heat transfer limitations within the heat pipe. We begin by addressing the heat transfer limit that results from the capillarity phenomena active in heat pipes. 


\section{Chapter 4. Capillary limit}

There are a number of heat and fluid flow phenomena that bound heat transfer within a heat pipe. Most relevant to liquid metal systems under hypersonic enthalpy conditions are: (i) the capillary limit, attained when the sum of vapor and liquid pressure drops along the heat pipe length matches the maximum capillary pressure which can be supported by the wick, (ii) the sonic limit, caused by a choking phenomenon when the vapor flow approaches Mach 1 and (iii) the boiling limit, reached when bubbles hinder fluid return flow. Whereas the previous chapter explored material limits, the present chapter examines limits which are affected by the working fluid behavior within the heat pipe.

Unfortunately, the standard capillary limit model for even a simple cylindrical system has struggled to predict experimental data [104], [105], [106]. The need for an improved model became apparent as a model-based leading edge design methodology was devised. Here, the governing principles of the standard capillary limit (the Chi model) theory are 
presented, and its deficiencies discussed. A modified model is developed for the simple case of a cylindrical system. A comparison of the new model predictions with experimental data from the literature reveals good agreement. This modified capillary limit model is then extended to a wedge-shaped leading edge geometry, and the impact of the capillary limit on leading edge design explored.

\subsection{Shortcomings of the Chi Model}

When the thermal flux applied to the exterior wall of a heat pipe reaches a critical level, the liquid-vapor interface can no longer be sustained in the wick due to a pressure imbalance across its surface. This gives rise to a dryout region: axial heat transfer along the pipe is no longer governed by evaporation and vapor flow, but by much less effective thermal conduction within the solid case material. Thermal hot spots then form with potential damage to the heat pipe.

The capillary limit was modeled by Chi [107] in 1976 and is the standard for heat pipe designers who seek an analytical tool for determining the maximum thermal flux that can be transported by a heat pipe. Of the few studies which have attempted to experimentally validate the Chi design model, most find that the experimentally determined limit lies well below the predicted values [104], [105], [106]. The model is therefore not 
conservative: its use could lead to unexpected failure under thermal conditions for which a heat pipe design was anticipated to successfully operate.

The basis of the Chi model can be described using the cylindrical heat pipe of Figure 4.1(a), with case wall thickness $b_{\text {wall }}$, wick thickness $b_{\text {wick }}$, and vapor space radius $R_{V}$. The heat pipe has an evaporator of length $L_{e}$ where heat applied to the wall is conducted in the through-thickness direction through the saturated wick to the liquid-vapor interface. The liquid evaporates in this region and carries the latent heat to the right through the vapor space adiabatic zone of length $L_{a}$, where no heat exchange with the external surroundings occurs. The vapor undergoes a phase change at the liquid-vapor interface in the condenser of length $L_{c}$, where it releases its latent heat which is conducted through the wick and wall, and into the surrounding medium (which is assumed cooler than that at the evaporator). The total tube length is $L_{t}=L_{e}+L_{a}+L_{c}$. At either end of the heat pipe is an insulating boundary so that all heat must enter or leave the circular surfaces of the pipe of the evaporator or condenser.

The curved liquid-vapor interface in the evaporator wick (Figure 4.1(b)) results from a pressure difference between the vapor pressure $P_{V}$ above the liquid surface and the liquid saturation pressure $P_{L}$. The Chi model employs a simplified version of the Laplace equation to relate the pressure difference across the interface to the liquid-vapor surface tension $\sigma_{L V}$ and the spherical meniscus radius of curvature $R_{m}$ [107]; 


$$
P_{V}-P_{L}=P_{C}
$$

where the capillary pressure $P_{C}$ is;

$$
P_{C}=\frac{2 \sigma_{L V}}{R_{m}}
$$

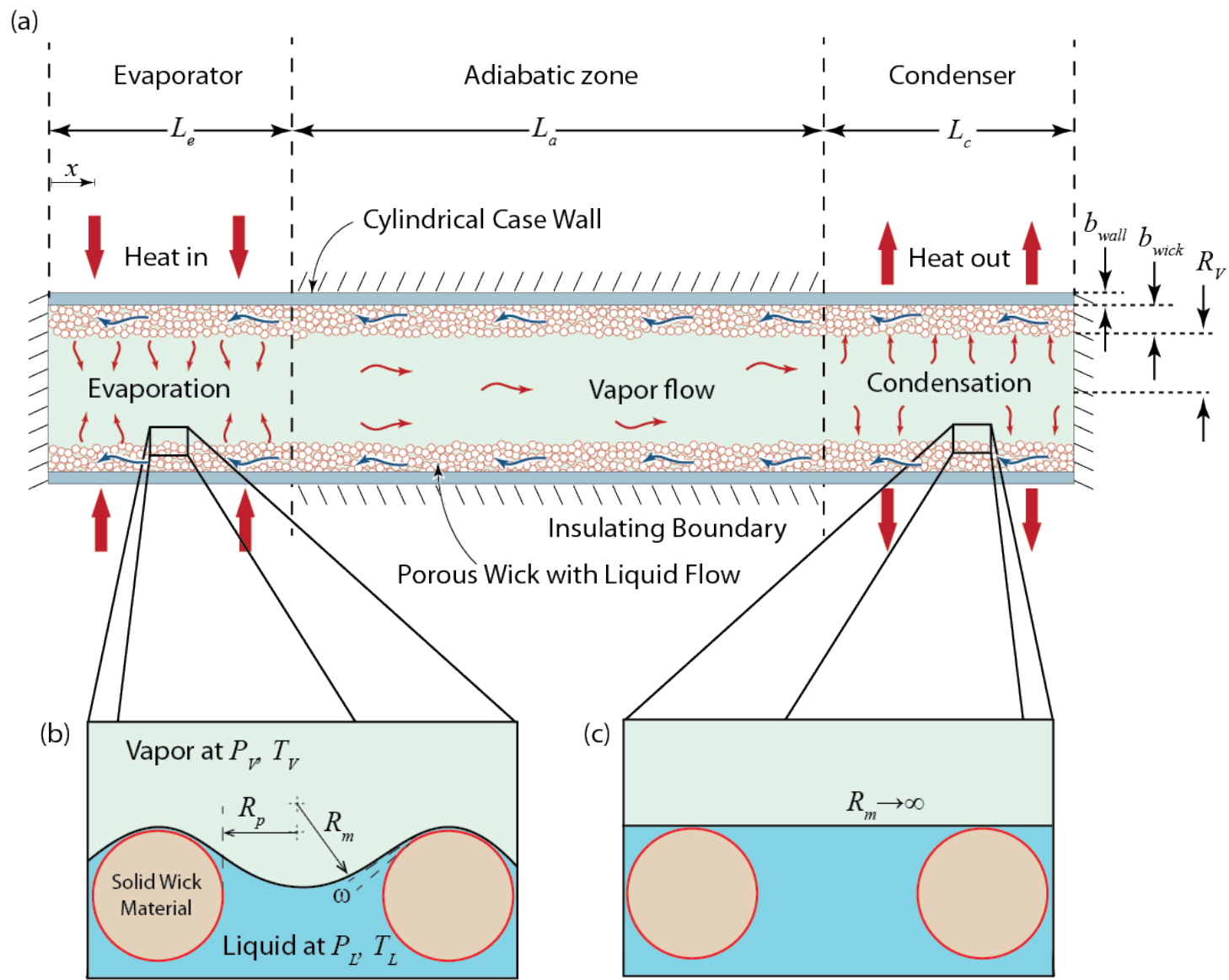

Figure 4.1. Cross-section view of a cylindrical heat pipe as represented by the Chi model. The evaporation/condensation cycle is shown in (a), with close-ups of the evaporator meniscus profile in (b) and condenser meniscus profile in (c). 
Heat pipes rely on pressure gradients established by the evaporation process to transport vapor (and thus the heat of evaporation) down the pipe from the evaporator to the condenser and within the working fluid to circulate the fluid back to the evaporation zone. At any location along the pipe, the difference in pressures must be balanced by surface forces at the liquid-vapor interface. The liquid and vapor pressure gradients which form as a result of the heat input in a steady state system are illustrated in Figure 4.2. The evaporator's elevated vapor pressure (as a result of evaporation) accelerates vapor molecules down the pipe toward the condenser where it decelerates, giving rise to a pressure recovery. At the condensation point, the liquid and vapor pressures are equal (due to flooding of the wick) and the interface is flat $\left(R_{m} \rightarrow \infty\right.$, see Figure 4.1(c)). The liquid pressure decreases in the direction from the condenser to the evaporator as liquid friction losses from the wick's interconnected but tortuous porous pathway accumulate. ${ }^{8}$ The net pressure drops over the respective vapor and liquid flow paths are caused by drag forces (friction) acting on the flows.

${ }^{8}$ There is also a pressure drop due to the acceleration of liquid molecules toward the evaporator, but this is negligible when compared to the viscous pressure drop. 


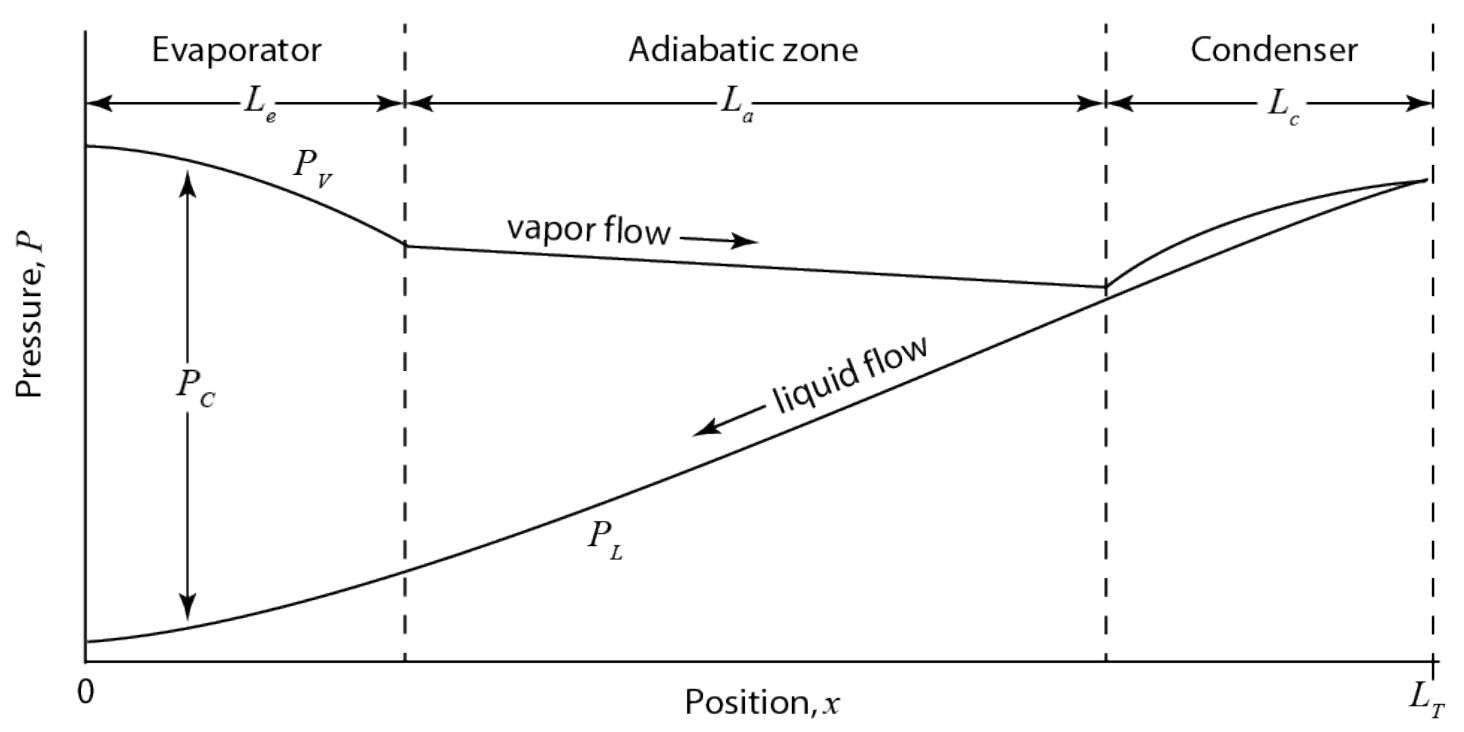

Figure 4.2. Pressure profiles for an operating heat pipe. Adapted from [73].

Because the minimum value of the meniscus radius, Figure 4.1 , is limited by the radius of the pore $R_{p}$, there is a maximum value for $P_{C}$. In the limit of $R_{m} \rightarrow R_{p}$;

$$
P_{C, \max }=2 \sigma_{L V} / R_{p}
$$

It follows from equation (4.1) that $P_{C, \max }$ determines the maximum allowable pressure difference across the interface. If $\left(P_{V}-P_{L}\right)>P_{C, \max }$, the interface becomes unstable and recedes into the wick leaving behind a region absent of liquid. This loss of liquid in the wick (and therefore evaporation) leads to a dryout condition characterized by thermal runaway there. 
For a cylindrical heat pipe, the Chi model argues that the capillary limit is reached when the maximum capillary pressure equals the cumulative pressure losses within the vapor and liquid flow paths of length $x$ [107];

$$
P_{C, \max } \geq \int_{x_{\min }}^{x_{\max }}\left(\frac{\partial P_{V}}{\partial x}-\frac{\partial P_{L}}{\partial x}\right) d x+\Delta P_{G}
$$

The vapor and liquid pressure gradients are given by [107];

$$
\frac{\partial P_{V}}{\partial x}=\frac{\left(f R e_{V}\right) \mu_{V}}{2 r_{h}{ }^{2} A_{V} \rho_{V} \lambda} Q(x)-\frac{\beta}{A_{V}{ }^{2} \rho_{V} \lambda^{2}} \frac{d Q(x)^{2}}{d x}
$$

and

$$
\frac{\partial P_{L}}{\partial x}=-\frac{\mu_{L}}{\rho_{L} \lambda A_{W} K} Q(x)
$$

where $\left(f \mathrm{Re}_{\mathrm{V}}\right)$ is the product of the vapor friction coefficient and Reynolds number, $\mu_{V}$ and $\mu_{L}$ are the vapor and liquid viscosities, $r_{h}$ is the hydraulic radius of the vapor space, $A_{V}$ and $A_{W}$ are the vapor space and wick cross-sectional areas, $\rho_{V}$ and $\rho_{L}$ are the vapor and liquid densities, $\lambda$ is the latent heat of vaporization/condensation, $K$ is the wick permeability, and $\beta$ is the momentum conservation factor and is a constant which depends on the vapor flow type (laminar or turbulent). The cumulative heat flow $Q(x)$ is positive (negative) if the direction of $Q(x)$ is opposite to (the same as) the direction of integration. The term $\Delta P_{G}$ accounts for the gravitational head on the liquid in the axial and radial directions. Locations $x_{\min }$ and $x_{\max }$ correspond to location of minimum and maximum values of the capillary 
pressure along the length of the heat pipe. For the pressure profile in Figure 4.2, these locations are at $x=0$ and $x=L_{t}$, respectively.

Representation of the liquid-vapor interface by the Chi model has been a source of concern for the model's validity. Figure 4.3(a) compares the interface inferred from kinetic theory [108] with that of the Chi model, Figure 4.3 (b). The kinetic theory of the liquidvapor interface distinguishes three regions: the bulk liquid, bulk vapor, and an interfacial region in which there is a steep density gradient over a distance of several atomic layers. A pressure and temperature is associated with each of these regions: $\left(P_{L}, T_{L}\right),\left(P_{V}, T_{V}\right)$, and $\left(P_{L V-E Q}, T_{L V}\right)$ [108]. The liquid-vapor interface is not well-defined; molecules continuously enter or leave the interfacial region which is driven by either a temperature or pressure jump (both can be present) from the bulk vapor. Interfacial transport theory [108], [109] shows that the net mass flux to the interface is proportional to $\left(\frac{P_{L V-E Q}}{T_{L V}{ }^{1 / 2}}-\frac{P_{V}}{T_{V}{ }^{1 / 2}}\right)$. When positive, the net flux of molecules is away from the surface and evaporation dominates. If the term is negative, condensation dominates. When no evaporation or condensation is taking place, the net mass flux of these molecules is zero. 


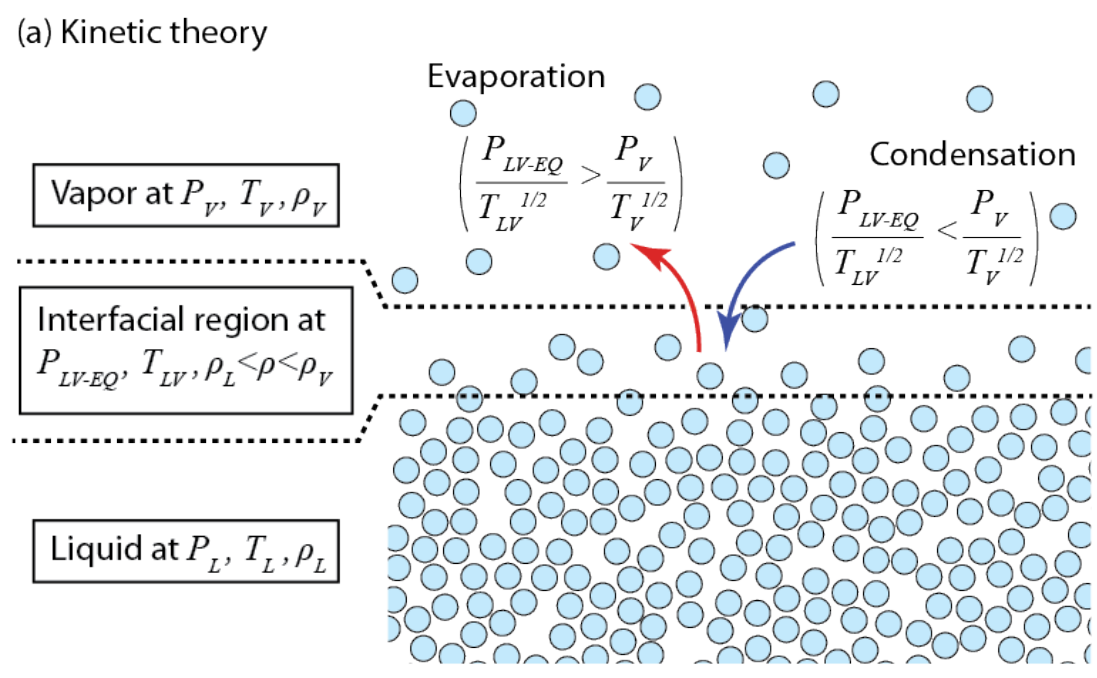

(b) Chi model

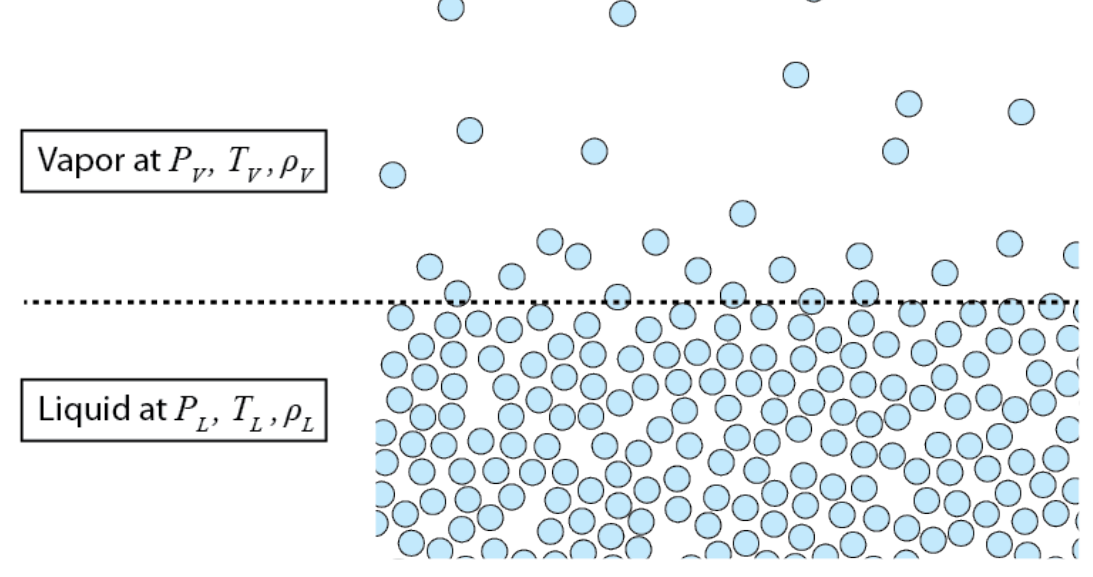

Figure 4.3. Molecular view of a liquid-vapor interface as represented by (a) kinetic theory and (b) the Chi model. The Chi model omits the interfacial region which is the active site for evaporation and condensation processes.

As shown in Figure 4.3(b), the Chi model assumes that the liquid-vapor interface conditions can be approximated by the vapor saturation conditions far from the interface, $P_{L V-E Q} \approx P_{V}$ and $T_{L V} \approx T_{V}$. The Chi model fails to account for the driving potential and 
therefore any mass transport across the interface. The assumption introduces only small errors at low evaporation rates since only small interfacial superheats and pressure jumps are present. At higher heat transport rates ${ }^{9}$, the pressure terms $P_{L V-E Q}$ and $P_{V}$ differ by nearly $10 \%$ [102]. The discrepancy results in an over-prediction of the capillary limit, the magnitude of which will be presented later.

An even more serious error [115] enters the analysis of short heat pipes designed to accommodate high heat fluxes. Numerous analytical, numerical, and experimental studies have shown that a wetting liquid meniscus has regions of active and in-active participation in phase change heat transfer [108], [109]. Figure 4.4 shows the profile of a wetting liquid meniscus in a wick. The central meniscus tapers down to a thin film at the wick ligament. The thin film can be subdivided into an evaporating region and non-evaporating, adsorbed film. The adsorbed film, with a thickness less than 100nm [116], experiences intermolecular attractions between the wick's solid material and the liquid surface giving rise to a disjoining pressure across the liquid-vapor interface [108], [111]. The disjoining pressure is inversely proportional to the cube of the wick thickness $\left(P_{D} \propto \delta^{-3}\right)$, so that as the thin flim thickness decreases, disjoining effects dominate capillary effects at the surface [108], [109], [117]. The disjoining pressure in the ultra thin film region disrupts the equilibrium interfacial pressure $P_{L V-E Q}$ by lowering its value. As a result, the ultra thin film exists in a superheated state

\footnotetext{
${ }^{9}$ In previous studies [110], [111], [112], [113], [114], superheats less than $6^{\circ} \mathrm{C}$ have been examined for evaporating non-alkali working fluids.
} 
without evaporation. This is reflected in the figure, which shows very little evaporation occurring at the adsorbed liquid surface. In contrast, the evaporating thin film region is characterized by higher heat transfer rates due to the short conduction pathway through the liquid to its surface (low thermal resistance) and diminished disjoining effects. The evaporation rate drops off dramatically in the intrinsic meniscus region as the liquid film thickness grows. Therefore, the rate of evaporating and condensing molecules depends on the curvature of the film and varies within a pore. The inclusion of disjoining effects, and a thin film region, localizes evaporation (condensation) to a small region of the liquid surface. Therefore, in order to maintain a heat balance with an external heat source, the driving potential (i.e. $\left.\left(\frac{P_{L V-E Q}}{T_{L V}{ }^{1 / 2}}-\frac{P_{V}}{T_{V}{ }^{1 / 2}}\right)\right)$ must be elevated above that for which evaporation (condensation) occurs over the heat pipe's entire evaporator (condenser). Because the interfacial mass flux has not been considered by Chi, this phenomenon is absent in his model. This has implications on the Laplace pressure balance (equation (4.1)) and the Chi model's governing equation (equation (4.4)).

Before proposing a modification to the Chi model for addressing the aforementioned deficiencies, a deeper discussion on the interaction of liquid and vapor molecules at an interface and the origin of surface tension is needed. 


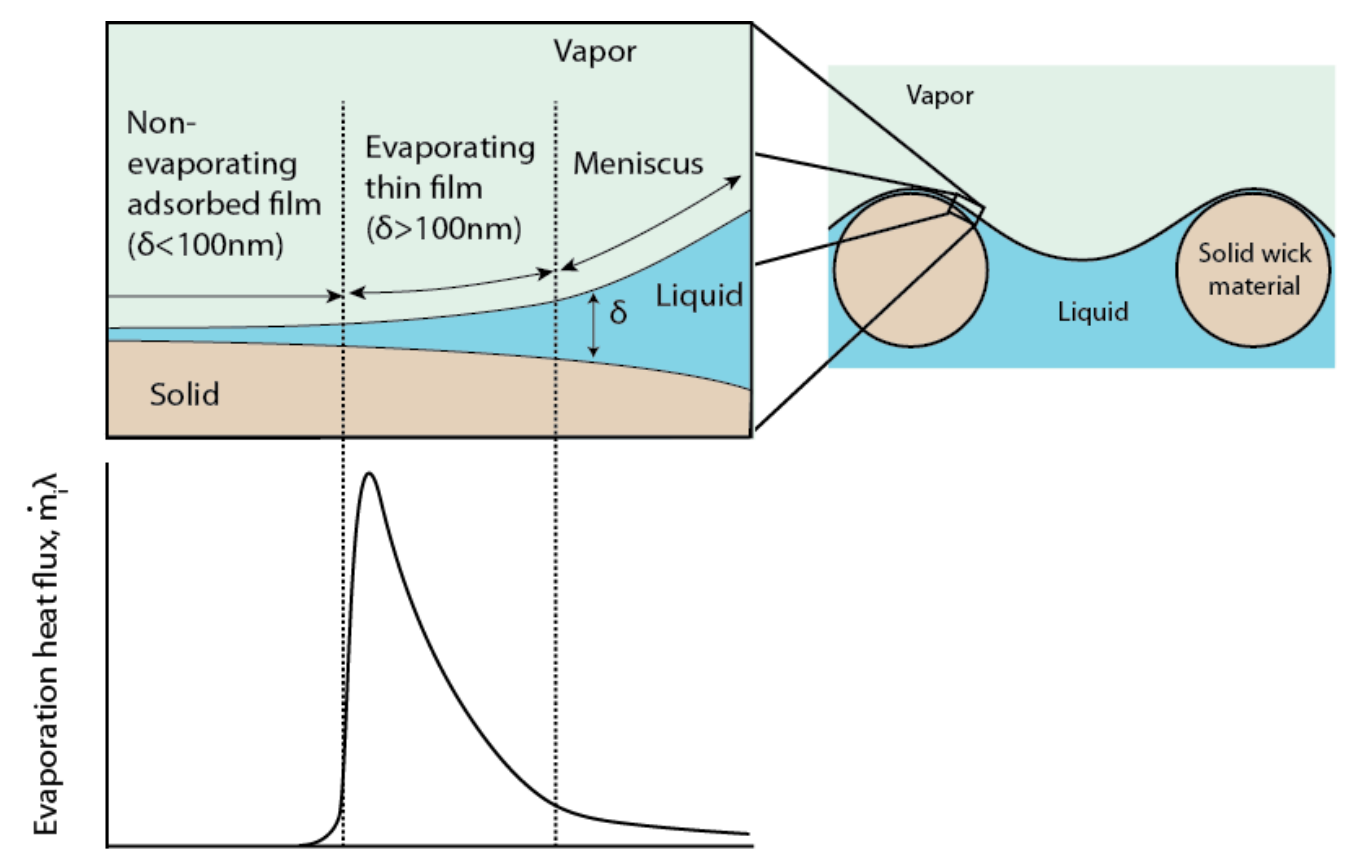

Film thickness, $\delta$

Figure 4.4. Liquid meniscus profile showing the variation in heat flux across a wick pore. Evaporation is suppressed at a small film thickness due to intermolecular attraction between the solid and liquid. It is likewise suppressed at the central meniscus which has a long thermal conduction pathway.

\subsection{Surface tension and the liquid-vapor interface}

In order to sustain the evaporation-condensation cycle within a heat pipe, the evaporator's working fluid must be replenished with that from the condenser. The porous wick provides a liquid return flow path that must remain saturated with fluid by sustaining a balance of forces at the liquid-vapor interface. The liquid's surface tension, $\sigma_{L V}$, plays the 
crucial role of supporting the wick's liquid against the elevated vapor pressure in the evaporator.

A simple atomistic model for the origin of the tension on a fluid's surface begins the development of the capillary limit theory. Figure 4.5(a) shows a schematic representation of the intermolecular potential between two molecules as a function of separation. It describes the binding energy between molecules at a given separation distance. At large distances (greater than 2.5 times the molecular diameter) [118], interaction between the molecules is negligible and the attractive potential is near zero. As the two molecules are brought closer together, the attractive potential becomes more negative as a result of van der Waal's interactions, exerting a stronger attractive force as shown in Figure 4.5(b). At a very short separation distance, the repulsive potential becomes large (as explained by the Pauli exclusion principle [119]) and a strong repulsive force pushes the molecules away from one another. The net sum of these potentials is the binding energy (solid black line) on which there exists a potential well (Figure 4.5(a)). In a crystalline solid, the intermolecular separation distance is well defined since their thermal energy is much smaller than the depth of the potential well: the molecules are in equilibrium at the bottom of the potential well, where the binding energy is minimized and the net force is zero (Figure 4.5(b)). Liquids, however, have a binding energy distributed within this well with no well-defined intermolecular separation (see blue shaded regions of Figure 4.5) [118]. 
(a)

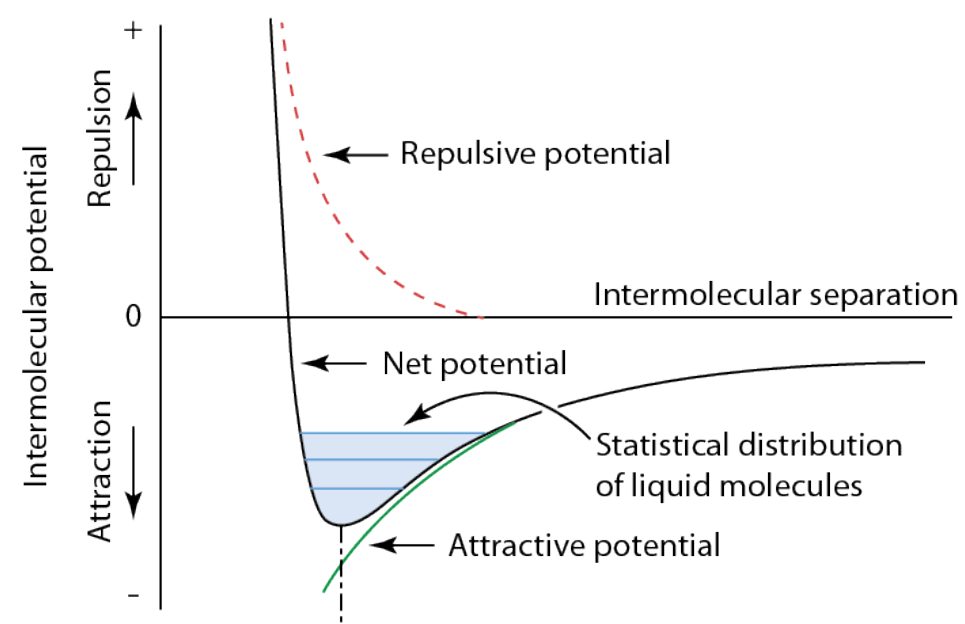

(b)

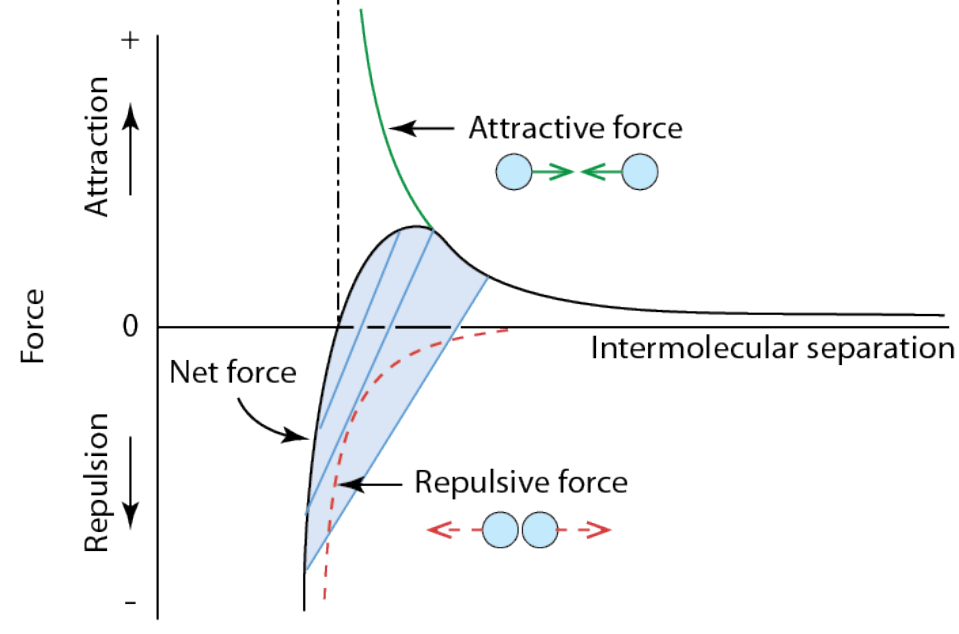

Figure 4.5. Schematic representation of (a) the intermolecular potential and (b) force as it depends on separation distance between two isolated molecules. The derivative of the potential gives the force. Adapted from [119].

A more complex interaction exists when considering many more molecules [119], but can be conceptualized using the same principles of the simpler two-molecule model. Consider the liquid-vapor system in Figure 4.6(a). The bulk liquid and vapor phases are separated by an interfacial region spanning several atomic layers [108]. The density of the 
interfacial region increases several orders of magnitude from the bulk vapor to the liquid surface (Figure 4.6(b)). Just below the liquid surface there is a shallow zone (highlighted in red) where the density continues to increase towards the liquid bulk density, $\rho_{L}$. The reduced density at the liquid surface results in an intermolecular force imbalance which gives rise to the surface tension. Figure 4.6(c) illustrates the attractive (solid green arrows) and repulsive forces (dashed red arrows) on molecules through a depth of several atomic layers.

We first consider only forces acting in the direction normal to the surface. Surface molecules experience attraction toward the bulk liquid only. Above the interface, the structure of the liquid breaks down: the summation of long range attractive forces from the interfacial region (being thin) and bulk gas (having low density) is small. The repulsive force must exactly balance this attraction as shown. At lower depths, more liquid molecules are found and the larger attractive force which results is balanced by the growing short range repulsive force which arises from the increasing liquid density. The attractive and repulsive forces reach their bulk liquid values at a depth where the density reaches the bulk value, $\rho_{L}$.

Now consider forces parallel to the surface, where long range attraction is nearly unaffected by changes in the liquid density. However, the short range repulsion parallel to the surface is strongly dependent on density. At the surface, the repulsive force is reduced by the increased intermolecular separation distance. The resultant force imbalance produces a net tensile force parallel to the surface: the surface tension, $\sigma_{L V}$. At larger depths, where the 
density approaches that of the bulk, the attractive and repulsive forces acting parallel to the surface balance [118].

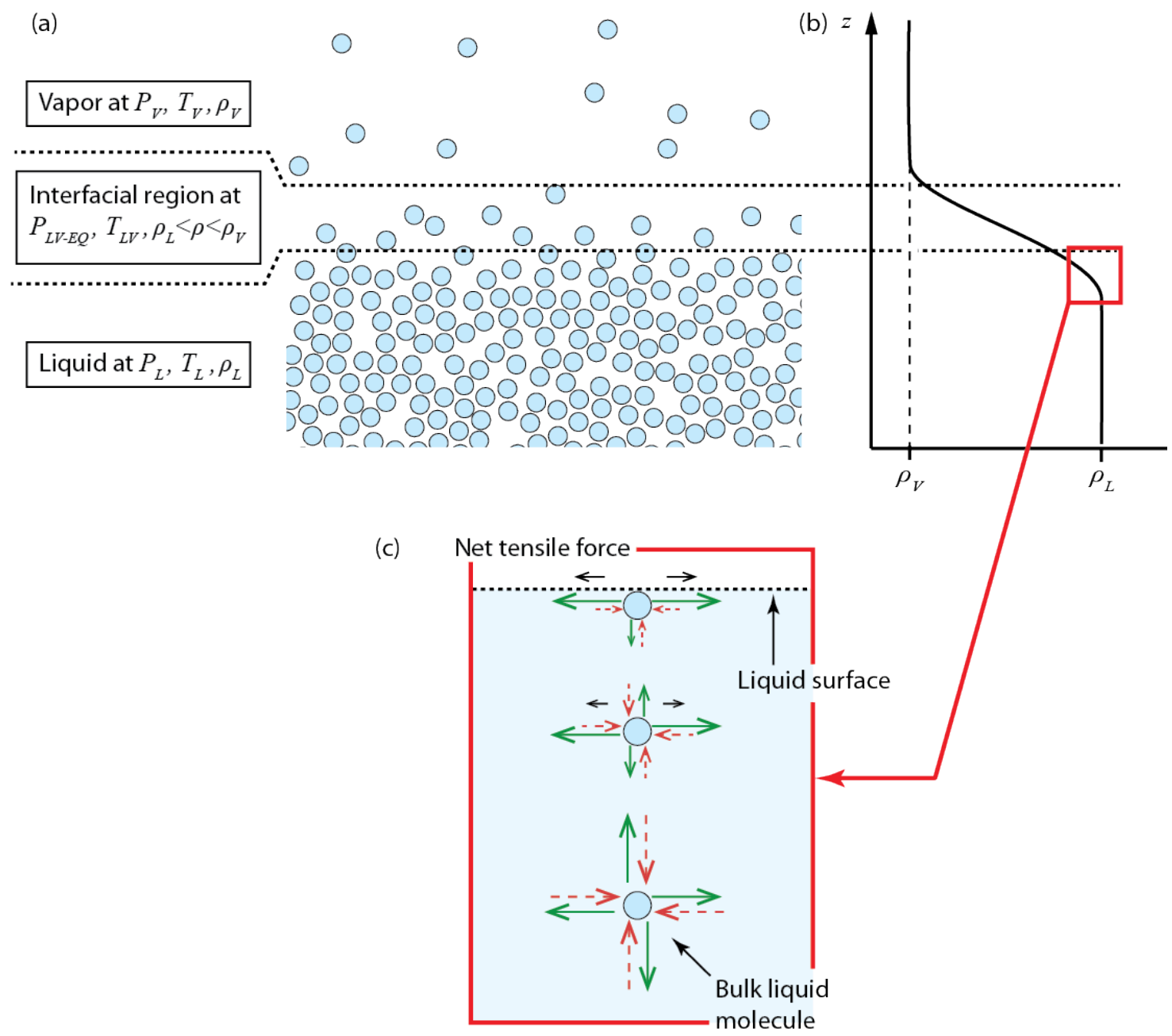

Figure 4.6. View of (a) the liquid-vapor interface showing three distinct regions whose fluid density is schematically plotted in (b). Near the liquid surface, forces of attraction $(\longrightarrow)$ and repulsion (-->) as a function of depth are shown in (c). Adapted from [108], [118]. 
A fluid's surface tension enables operation of a heat pipe by maintaining wick saturation when a localized region of elevated vapor pressure develops during heating. Consider a wick's network of pores lining the interior of the heat pipe wall. The wick is saturated with a working fluid whose liquid surface is in contact with its own vapor. Forces acting on the liquid-vapor interface are shown in Figure 4.7. The net attractive force acting on the liquid toward the vapor, $\boldsymbol{F}_{\boldsymbol{L}}$, and the force exerted on the liquid surface by the vapor above the interface, $\boldsymbol{F}_{\boldsymbol{L} \boldsymbol{V}-\boldsymbol{E} \boldsymbol{Q}}$, are given by;

$$
\begin{gathered}
\boldsymbol{F}_{\boldsymbol{L}}=\pi R_{p}^{2} P_{L} \\
\boldsymbol{F}_{\boldsymbol{L} \boldsymbol{V}-\boldsymbol{E} \boldsymbol{Q}}=-\pi R_{p}^{2} P_{L V-E Q}
\end{gathered}
$$

where $R_{p}$ is the wick pore radius, $P_{L}$ is the liquid pressure, and $P_{L V-E Q}$ is the equilibrium vapor pressure in the interfacial region. The figure defines the unit normal vector, $\widehat{\boldsymbol{n}}$, which gives rise to the negative sign on the right hand side of equation (4.8). The vertical component of the capillary force exerted on the liquid's surface is;

$$
\boldsymbol{F}_{\boldsymbol{C}}=2 \pi R_{p} \sigma_{L V} \cos \omega
$$

where $R_{p}$ is the wick pore radius and $\omega$ is the contact angle at the solid ligament which, by geometry, can be expressed as the ratio of the two radii;

$$
\cos \omega=\frac{R_{p}}{R_{m}}
$$


In equation (4.10) we have adopted the convention that the meniscus radius of curvature $R_{m}$ is positve if the liquid-vapor interface curves towards the vapor, as it does here. Substituting equation (4.10) into (4.9) gives the capillary force as;

$$
\boldsymbol{F}_{\boldsymbol{C}}=2 \pi \sigma_{L V} \frac{R_{p}{ }^{2}}{R_{m}}
$$

A force balance maintains equilibrium to keep the liquid surface suspended in the wick;

$$
\boldsymbol{F}_{L}+\boldsymbol{F}_{\boldsymbol{L}-\boldsymbol{E Q}}+\boldsymbol{F}_{\boldsymbol{C}}=0
$$

Substituting for each force component using equations (4.7), (4.8), and (4.11);

$$
P_{L V-E Q}-P_{L}=P_{C}
$$

where $P_{C}$ is the capillary pressure given by $P_{C}=2 \sigma_{L V} / R_{m}$. Equation (4.13) is the Laplace equation [109], which relates the pressure difference across an interface to its surface tension and curvature. When the pressure difference $\left(P_{L V-E Q}-P_{L}\right)$ is balanced by the capillary pressure $P_{C}$, the liquid remains suspended in the pore. 


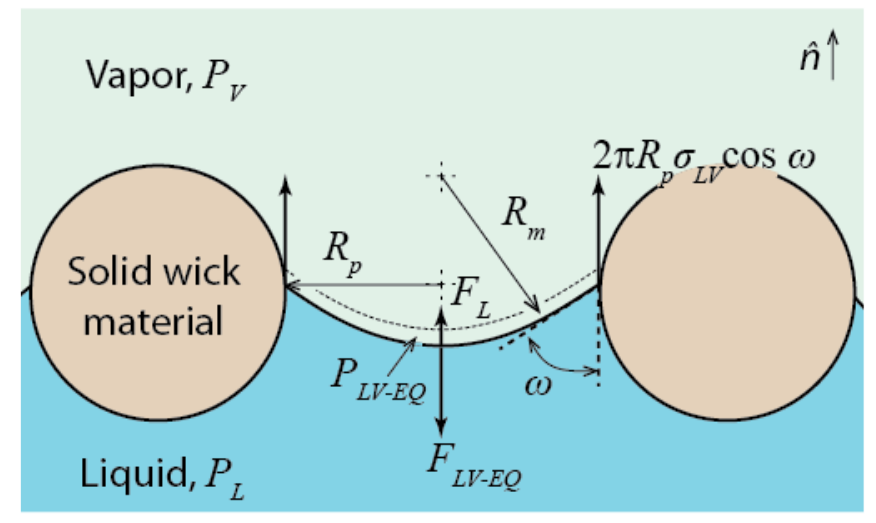

Figure 4.7. Free body diagram of a pore's liquid meniscus.

Heat pipes rely on pressure gradients to transport vapor from the evaporator to the condenser and to circulate the fluid back to the evaporation zone. At any location along the pipe, the difference in the interfacial region pressure and the liquid pressure must be balanced by surface forces at the liquid-vapor interface. Because the minimum value of the meniscus radius is limited by the size of the pore, there is a maximum value for $P_{C}$. In the limit of $R_{m} \rightarrow R_{p}$

$$
P_{C, \max }=2 \sigma_{L V} / R_{p}
$$

If $\left(P_{L V-E Q}-P_{L}\right)>P_{C, \max }$, the interface becomes unstable and recedes into the wick leaving behind a region absent of liquid, causing the dryout condition. The size of a wick's pores - be it a foam, screen, felt, or sintered powder - plays a critical role in a heat pipe's operation. 
The capillary limit model establishes the relationship between the maximum applied heat and pressure balance at the liquid-vapor interface. There are two advantages in first developing the model for a simpler cylindrical pipe;

(i) the cylindrical concept has a constant cross-sectional area, simplifying the vapor flow equations; and,

(ii) experimental data for a cylindrical pipe are available in the literature for model validation.

Its concepts and equations lay the foundation for the wedge-shaped system presented later.

\subsection{Modified Chi Model (Cylindrical Pipe)}

To address limitations of the Chi model for a cylindrical heat pipe, a solvable system of equations that accounts for the variation of the interfacial mass flux at the meniscus surface is developed.

\section{3(a) Assumptions}

The model is derived for the heat pipe shown in Figure 4.8. As before, there is an evaporator, adiabatic section, and condenser with respective lengths $L_{e}, L_{a}$, and $L_{c}$, and a total length of $L_{t}=L_{e}+L_{a}+L_{c}$. Heat exchange with the external environment is 
allowed only through the curved surface of the evaporator and condenser (the circular ends are taken to be perfect insulators).

The following assumptions are made for the vapor stream:

(i) Constant velocity profiles: The velocity distribution across the vapor core crosssection is approximated by a constant velocity profile, so that the velocity is taken to be an average velocity.

(ii) No slip upstream and downstream: A no slip condition (axial vapor velocity is zero) is assumed at the upstream end of the evaporator $(x=0)$ and the downstream end of the condenser $\left(x=L_{t}\right) 10$. A stagnation condition persists at these points.

(iii) Normal injection velocity: The direction of the velocity vector for the injected/suctioned mass is perpendicular to the flow direction, and therefore contributes no momentum in the direction of the vapor flow.

\section{3(b) Derivation}

As before, the liquid, vapor, and gravitational pressure drops along the length of the heat pipe are balanced by the capillary pressure difference using the Laplace equation;

${ }^{10}$ A no-slip condition at $x=0$ and $L_{t}$ is also assumed for liquid flow. 


$$
\left.\Delta P_{C}\right|_{x} ^{0}=\left.\Delta P_{L V-E Q}\right|_{x} ^{0}+\left.\Delta P_{L}\right|_{0} ^{x}+\Delta P_{G}
$$

Noting at the capillary limit that $R_{M} \rightarrow R_{P}$ in the evaporator, then $P_{C}(x=0) \rightarrow$ $P_{C, \max }=2 \sigma_{L V} / R_{P}$. Substituting this into equation (4.15) gives;

$$
\begin{gathered}
P_{C}(x)=P_{L V-E Q}(x)-P_{L V-E Q}(x=0)+\frac{2 \sigma_{L V}}{R_{P}}-\left.\Delta P_{L}\right|_{0} ^{x} \\
-\Delta P_{G}(x)
\end{gathered}
$$

The liquid $\left.\Delta P_{L}\right|_{0} ^{x}$ and hydrostatic pressure $\Delta P_{G}$ drops are given by;

$$
\left.\Delta P_{L}\right|_{0} ^{x}=\frac{\mu_{L}}{\rho_{L} \lambda A_{W} K} \int_{0}^{x} Q d x
$$

and

$$
\Delta P_{G} \approx 2 \rho_{L} g R_{V}
$$

Note that the liquid pressure drop in (4.17) is identical to the one used in the Chi model. 


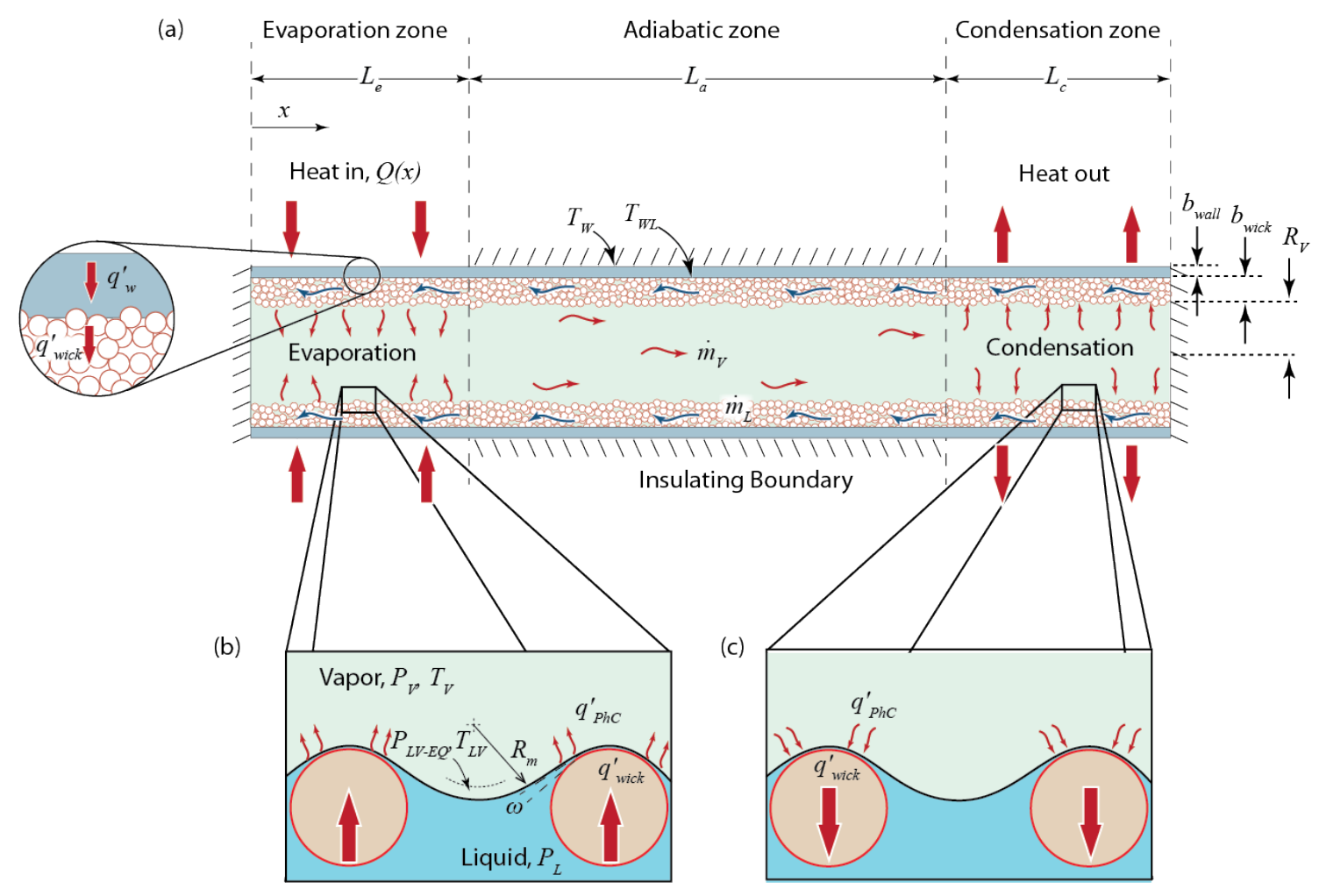

Figure 4.8. The heat pipe used for the modified Chi model is shown in (a), with close-up views of the (b) evaporator meniscus and (c) condenser meniscus.

Consider the flat (a) and not flat (b) liquid-vapor interfaces shown in Figure 4.9. The bulk liquid density is identical in both cases, so the long range attractive force (van der Waals) acting to attract interfacial molecules toward the liquid are the same for each interface. However, because there are more liquid molecules at the curved surface than the flat one, the repulsive forces are stronger. To maintain the force balance on the interfacial molecule, the intermolecular spacing from the curved surface is slightly increased, having the effect of reducing the repulsive forces with little change in the long range attractive forces. 
The result is a lower interfacial density and vapor pressure $P_{L V-E Q}$ over that above the flat interface $P_{L V}$. The relationship between $P_{L V-E Q}$ and $P_{L V}$ is given by the Kelvin equation [109]:

$$
P_{L V-E Q}=P_{L V} \times \operatorname{Exp}\left[\frac{-M P_{C}}{\rho_{L} R T_{L V}}\right]
$$

where $M$ is the molar mass of the fluid, $R$ is the universal gas constant $\left(\approx 8.314 \mathrm{JK}^{-1} \mathrm{~mol}^{-1}\right)$, and $T_{L V}$ is the saturation interface temperature. The term $\left[-M P_{C} / \rho_{L} R T_{L V}\right]$ raises or lowers the equilibrium vapor pressure as a result of the interface curvature prescribed by $P_{C}$. If $P_{C}$ is positive (positive curvature), then the vapor pressure is lowered compared to that over a flat interface. If $P_{C}$ is negative (negative curvature), then it is raised. The implication is that the interfacial region above a liquid meniscus with positive curvature is in a slightly superheated state. The vapor pressure at the flat interface at temperature $T_{L V}$ can be determined from the Clausius-Clayperon equation [108];

$$
P_{L V}=P_{V, R E F} \times \operatorname{Exp}\left[\frac{M \lambda}{R}\left(\frac{1}{T_{V, R E F}}-\frac{1}{T_{L V}}\right)\right]
$$

where $\lambda$ is the latent heat of the fluid, and $P_{V, R E F}$ and $T_{V, R E F}$ are a reference saturation pressure and temperature, respectively, and depend on the type of working fluid. Values can 
be found for several working fluids in Appendix B. Substituting equation (4.20) into (4.19) gives;

$$
P_{L V-E Q}=P_{V, R E F} \times \operatorname{Exp}\left[\frac{M\left(\lambda \rho_{L}\left(T_{L V}-T_{V, R E F}\right)-P_{C} T_{V, R E F}\right)}{\rho_{L} R T_{L V} T_{V, R E F}}\right]
$$

While equation (4.21) has provided an expression for $P_{L V-E Q}$, it has also introduced a new unknown, $T_{L V}$, and it is to that we now turn.

(a) Flat interface

$$
P_{L V}
$$

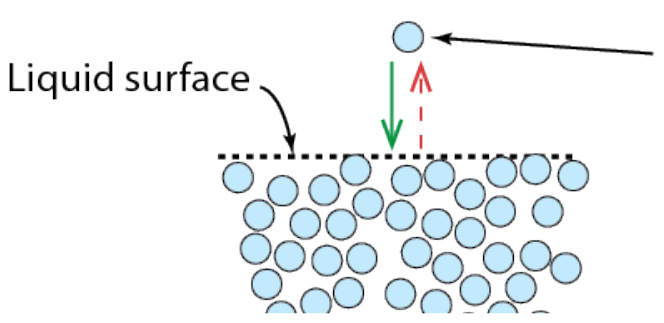

(b) Curved interface

$P_{L V-E Q}$

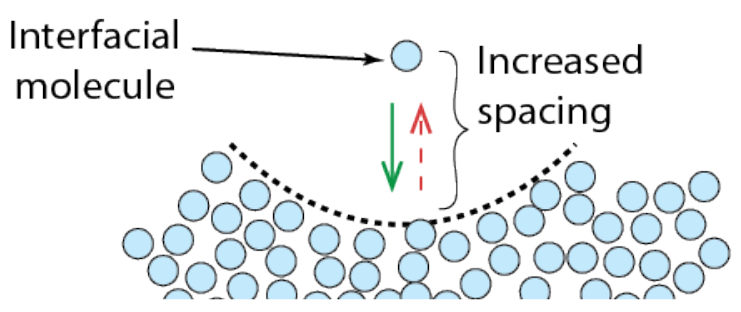

Figure 4.9. Force balance for an interfacial molecule at a (a) flat and (b) curved interface. An increase in intermolecular spacing above the curved surface maintains the force balance.

Using Fourier's law of conduction in cylindrical coordinates [103], the heat transfer rate per unit length through the case wall is;

$$
q^{\prime}{ }_{w}=2 \pi k_{w a l l} \frac{T_{W L}-T_{W}}{\ln \left[\frac{R_{\text {wall }}}{R_{\text {wall }}-b_{\text {wall }}}\right]}
$$

and that through the thickness of the wick is; 


$$
q^{\prime}{ }_{\text {wick }}=2 \pi k_{\text {wick }} \frac{T_{L V}-T_{W L}}{\ln \left[\frac{R_{\text {wall }}-b_{\text {wall }}}{R_{V}}\right]}
$$

where $q$ and $k$ are the heat transfer rate per unit length and thermal conductivity, respectively, $T_{W}$ is the temperature at the outer surface of the wall, and $T_{W L}$ is the temperature at the wall/liquid interface. The case radius is given by $R_{\text {wall }}=b_{\text {wall }}+$ $b_{w i c k}+R_{V}$. For heat pipes, the heat flux along the wall and wick is much lower than in the through thickness direction [94]. We can therefore set $q^{\prime}{ }_{w}=q^{\prime}{ }_{w i c k}$ and solve for $T_{L V}$;

$$
T_{L V}=\left(T_{W L}-T_{W}\right)\left(\frac{k_{\text {wall }}}{k_{\text {wick }}}\right)\left(\frac{\ln \left[\frac{R_{\text {wall }}-b_{\text {wall }}}{R_{V}}\right]}{\ln \left[\frac{R_{\text {wall }}}{R_{\text {wall }}-b_{\text {wall }}}\right]}\right)+T_{W L}
$$

An additional unknown temperature, $T_{W L}$, has now been introduced. An equation for the phase change heat transfer rate per unit length $q^{\prime}{ }_{P h C}$ at the liquid-vapor interface can be expressed as a function of the latent heat, $\lambda$, and average evaporation/condensation mass flux $\bar{m}_{i}$;

$$
q_{P h C}^{\prime}=2 \pi R_{V} \lambda \bar{m}_{i}
$$

The heat balance at the liquid-vapor interface allows equations (4.23) and (4.25) to be equated. Solving for $T_{W L}$ in terms of $\bar{m}_{i}$ provides; 


$$
T_{W L}=\frac{R_{V} \lambda \bar{m}_{i}}{k_{\text {wick }}} \ln \left[\frac{R_{\text {wall }}-b_{\text {wall }}}{R_{V}}\right]+T_{L V}
$$

At steady state, heat which is transported by the heat pipe must evolve into an evaporation and condensation event (i.e. the net rate of energy storage in the case, liquid, or vapor is zero). Therefore, $\bar{m}_{i}$ can be expressed directly as a function of the input power $Q$;

$$
\bar{m}_{i}=\frac{1}{2 \pi \lambda R_{V}} \frac{d Q}{d x}
$$

which upon substituting into equation (4.26) completes the expression for $T_{W L}$;

$$
T_{W L}=\frac{1}{2 \pi k_{\text {wick }}} \ln \left[\frac{r_{\text {wall }}-b_{\text {wall }}}{r_{\text {vapor }}}\right] \frac{d Q}{d x}+T_{L V}
$$

We now turn our attention to the vapor flow. Conservation of axial vapor momentum gives (see Appendix C for full derivation);

$$
d \mathrm{P}_{V}=-\frac{\beta}{\rho_{V} \lambda^{2} A_{V}^{2}}\left(2 Q d Q-\frac{Q^{2}}{\rho_{V}} d \rho_{V}\right)-\frac{\mu_{V} f R e_{V} Q}{8 \lambda \rho_{V} A_{V} R_{V}^{2}} d x
$$

The terms (from left to right) on the right hand side account for the inertial, compressible, and viscous effects respectively. Analytical integration is non-trivial; therefore, equation (4.29) was discretized using a forward difference scheme, posing it in algebraic form for solving the system of equations. 
To complete the system of equations, the Schrage equation is used to relate the saturation vapor properties and those at the liquid-vapor interface with the local interfacial mass flux [108], [109];

$$
\dot{m}_{i}=\frac{2 c}{2-c}\left(\frac{M}{2 \pi R}\right)^{1 / 2}\left(\frac{P_{L V-E Q}}{T_{L V}^{1 / 2}}-\frac{P_{V}}{T_{V}^{1 / 2}}\right)
$$

where $\dot{m}_{i}$ is the local evaporation/condensation rate per unit area of the active thin film region as depicted in Figure 4.10.

An expression is needed which relates $\dot{m}_{i}$ to the average mass flux $\bar{m}_{i}$ of equation (4.27). It is here that we remedy the second model deficiency. As depicted in the figure, we define a surface area of active evaporation/condensation which is projected onto a plane parallel to the wick surface, $A_{A}$, and a typical cell surface area, $A_{C}$. Let an areal fraction $F$ be defined as;

$$
F=A_{A} / A_{C}
$$

such that, using a mass balance, the local mass flux $\dot{m}_{i}$ can be described by;

$$
\dot{m}_{i}=\bar{m}_{i} /_{F}
$$


Defining $\dot{m}_{i}$ in this manner has the effect of increasing it over the unit cell average. Substituting equation (4.32) into (4.30) provides an expression for the average evaporation/condensation flux;

$$
\bar{m}_{i}=\frac{2 c}{2-c}\left(\frac{M}{2 \pi R}\right)^{1 / 2}\left(\frac{P_{L V-E Q}}{T_{L V}{ }^{1 / 2}}-\frac{P_{V}}{T_{V}^{1 / 2}}\right) F
$$

which can be expressed in terms of the vapor heat flow (using equation (4.27));

$$
\frac{d Q}{d x}=\frac{2 c}{2-c}\left(\frac{M}{2 \pi R}\right)^{1 / 2}\left(\frac{P_{L V-E Q}}{T_{L V}{ }^{1 / 2}}-\frac{P_{V}}{T_{V}^{1 / 2}}\right) 2 \pi \lambda F R_{V}
$$




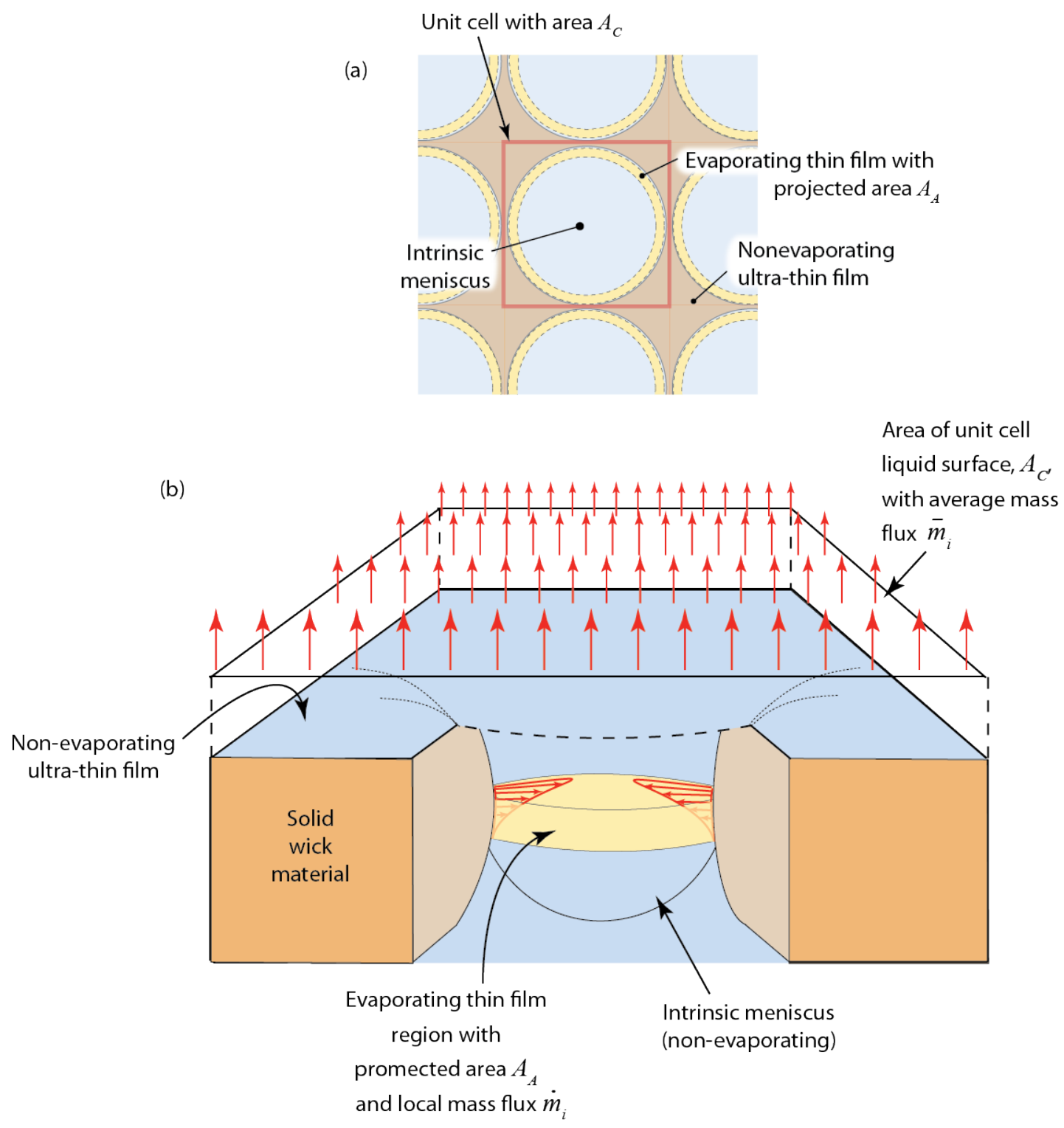

Figure 4.10. Schematic of the meniscus interface. An elevated view in (a) shows the repeating unit cell pore with area $A_{C}$. The wick's unit cell, shown in (b), has evaporation occurring with local mass flux $\dot{m}_{i}$ over the thin film region of projected area $A_{A}$. The local mass flux $\dot{m}_{i}$ (mass per unit time per unit area) can be represented as a unit cell mass flux $\bar{m}_{i}$ over $A_{C}$ with an equivalent evaporation rate (mass per unit time). 
The system of equations is completed with the perfect gas and Clausius Clapeyron equations for the vapor [97];

$$
\begin{gathered}
P_{V}=\frac{\rho_{V} R T_{V}}{M} \\
P_{V}=P_{V, R E F} \times \operatorname{Exp}\left[\frac{M \lambda}{R}\left(\frac{1}{T_{V, R E F}}-\frac{1}{T_{V}}\right)\right]
\end{gathered}
$$

A summary of the nine equations and variables are presented in Table 4.1. 
Table 4.1. The nine equation system and variables for the modified Chi model of the cylindrical heat pipe.

\begin{tabular}{|c|c|c|c|}
\hline Name & Equation number & Equation & $\begin{array}{l}\text { Unknown } \\
\text { variable }\end{array}$ \\
\hline Full Laplace & $(4.16)$ & $P_{C}(x)=P_{L V-E Q}(x)-P_{L V-E Q}(x=0)+\frac{2 \sigma_{L V}}{R_{P}}-\left.\Delta P_{L}\right|_{0} ^{x}-\Delta P_{G}$ & $P_{L V-E Q}$ \\
\hline Liquid pressure drop & $(4.17)$ & $\left.\Delta P_{L}\right|_{0} ^{x}=\frac{\mu_{L}}{\rho_{L} \lambda A_{W} K} \int_{0}^{x} Q d x$ & $\Delta P_{L}$ \\
\hline Kelvin equation & $(4.21)$ & $P_{L V-E Q}=P_{V, R E F} \times \operatorname{Exp}\left[\frac{M\left(\lambda \rho_{L}\left(T_{L V}-T_{V, R E F}\right)-P_{C} T_{V, R E F}\right)}{\rho_{L} R T_{L V} T_{V, R E F}}\right]$ & $P_{C}$ \\
\hline $\begin{array}{l}\text { Wall/Wick heat } \\
\text { balance }\end{array}$ & $(4.24)$ & $T_{L V}=\left(T_{W L}-T_{W}\right)\left(\frac{k_{\text {wall }}}{k_{\text {wick }}}\right)\left(\frac{\ln \left[\frac{R_{\text {wall }}-b_{\text {wall }}}{R_{V}}\right]}{\ln \left[\frac{R_{\text {wall }}}{R_{\text {wall }}-b_{\text {wall }}}\right]}\right)+T_{W L}$ & $T_{L V}$ \\
\hline $\begin{array}{l}\text { Wick/Vapor heat } \\
\text { balance }\end{array}$ & $(4.26)$ & $T_{W L}=\frac{R_{V} \lambda \bar{m}_{i}}{k_{w i c k}} \ln \left[\frac{R_{\text {wall }}-b_{\text {wall }}}{R_{V}}\right]+T_{L V}$ & $T_{W}$ \\
\hline Schrage Equation & $(4.34)$ & $\frac{d Q}{d x}=\frac{2 c}{2-c}\left(\frac{M}{2 \pi R}\right)^{1 / 2}\left(\frac{P_{L V-E Q}}{T_{L V}{ }^{1 / 2}}-\frac{P_{V}}{T_{V}^{1 / 2}}\right) 2 \pi \lambda F R_{V}$ & $T_{W L}$ \\
\hline
\end{tabular}


Vapor momentum

Perfect Gas

Clausius Clayperon

(4.29)

$$
d P_{V}=-\frac{\beta}{\rho_{V} \lambda^{2} A_{V}^{2}}\left(2 Q d Q-\frac{Q^{2}}{\rho_{V}} d \rho_{V}\right)-\frac{\mu_{V} f R e_{V} Q}{8 \lambda \rho_{V} A_{V} R_{V}^{2}} d x
$$

$$
P_{V}=\frac{\rho_{V} R T_{V}}{M}
$$

$P_{V}$

$\rho_{V}$ 


\section{3(c) Boundary Conditions}

If we assume an applied heat flux which is constant over the evaporator and condenser, then the cumulative heat in the vapor, $Q$ can be represented by the linear function;

$$
Q(x)=\left\{\begin{array}{cc}
\frac{x}{L_{e}} Q_{\max } & 0 x<L_{e} \\
Q_{\max } & L_{e} \leq x<L_{e}+L_{a} \\
\left(\frac{L_{t}-x}{L_{c}}\right) Q_{\max } & L_{e}+L_{a} \leq x \leq L_{t}
\end{array}\right.
$$

where $Q_{\max }$ is the maximum transported power. ${ }^{11}$ Figure 4.11 shows how $Q$ varies with axial position.

11 A polynomial function was used to approximate the cumulative heat flow for the evaporator to adiabatic zone transition, and the adiabatic to condenser transition. This enforces a continuous derivative $d Q / d x$. 


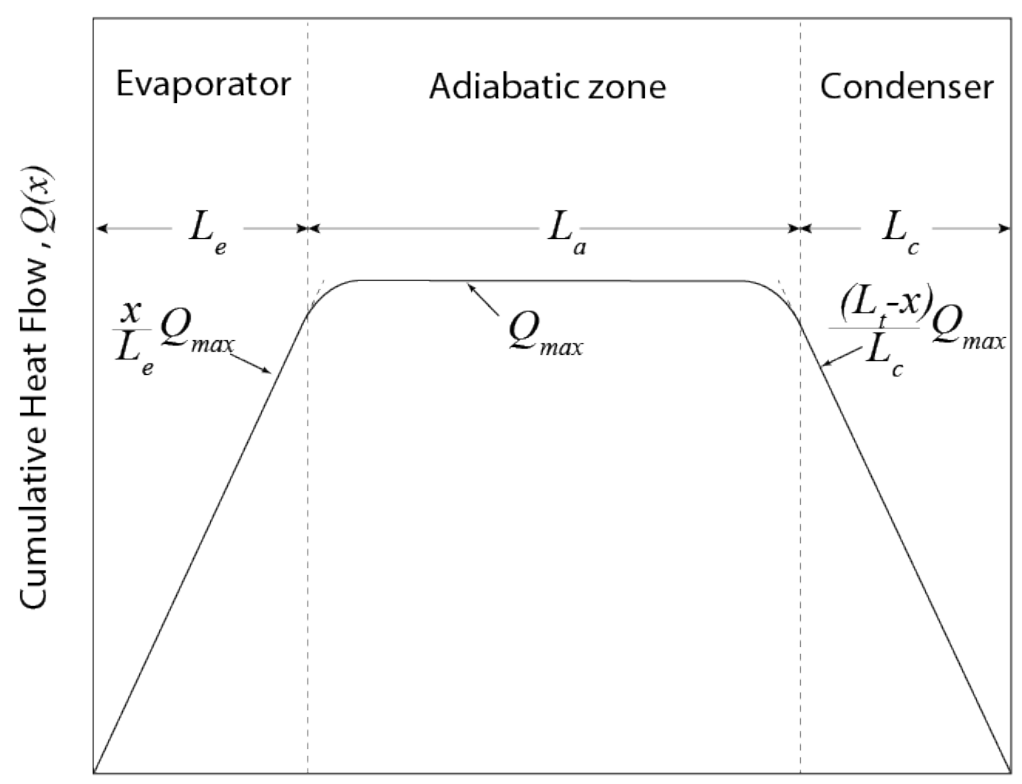

Axial position, $x$

Figure 4.11. Applied cumulative heat flow through the length of the heat pipe.

\section{3(d) Solution Method}

Wolfram Mathematica was used for solving the system of equations. The solution algorithm, mapped in Figure 4.12, is:

1. Incompressible, laminar vapor flow is tentatively assumed with assigned values of $f \mathrm{Re}_{\mathrm{V}}=64$ and $\beta=4 / 3$.

2. A vapor saturation temperature and pressure $\left(T_{V, o}, P_{V}\right)$ is chosen and an initial value for $Q_{\max }$ is guessed. 
3. The Kelvin and Schrage equations are simultaneously solved for $P_{L V-E Q}$ and $T_{L V}$ at $x=0$ where $P_{C}=P_{C, \max }$.

4. The solution for $P_{L V-E Q}(x=0)$ is used in the Laplace equation (see equation (4.16)) and the nine equation system is numerically solved.

5. The maximum vapor Reynolds number, given by ;

$$
R e_{V}=\frac{2 R_{V} Q_{\max }}{A_{V} \mu_{V} \lambda}
$$

is calculated to determine if the laminar flow assumption was valid. Also, the maximum vapor Mach number, given by;

$$
M_{V}=\frac{Q_{\max }}{A_{V} \rho_{V} \lambda \sqrt{\gamma_{V} R T_{V}}}
$$

is calculated to determine if the incompressible flow assumption was valid. In equation (4.39), $\gamma_{V}$ is the vapor's heat capacity ratio and $T_{V}$ should be evaluated at $x=L_{e}$. If $\operatorname{Re}_{\mathrm{V}} \leq 2300$ and $M_{V} \leq 0.3$, the laminar and incompressible assumption is valid and the tentative values for $f \operatorname{Re}_{\mathrm{V}}$ and $\beta$ are valid. If $\operatorname{Re}_{\mathrm{V}}>2300$, then the flow is turbulent and the momentum correction coefficient is reassigned a value of $\beta=1$ [93]. $f$ Re is recalculated using the following empirical relation for turbulent flow [120];

$$
f R e_{V}=0.152 R e_{V}^{0.75}
$$


If $M_{V}>0.3$, then the flow must be treated as a compressible fluid, and $f R e_{V}$ should be multiplied by a conversion factor [107];

$$
\frac{f_{c}}{f}=\left(1+\frac{\gamma_{V}-1}{2} M_{V}^{2}\right)^{-1 / 2}
$$

where $f_{c}$ is the compressible friction factor and $M_{V}$ is evaluated at $x=L_{e}$. These new values for $\beta$ and/or $f \operatorname{Re}_{\mathrm{V}}$ are used to recalculate $P_{L V-E Q}(x=0)$ (step 3 ) and solve again the nine-equation system (step 4).

6. Once it has been determined that correct values have been used for flow variables $\beta$ and $f$ Re, the solution for $P_{C}$ is evaluated to determine if the guessed value $Q_{\max }$ is indeed the limit. If $P_{C}>0$ everywhere ( $P_{C}<0$ anywhere) then $Q_{\max }$ is increased (decreased) and the solution algorithm is iterated from step 2. The limit has been converged upon once $P_{C}=0$ anywhere and $P_{C}>0$ everywhere else along the pipe length. 


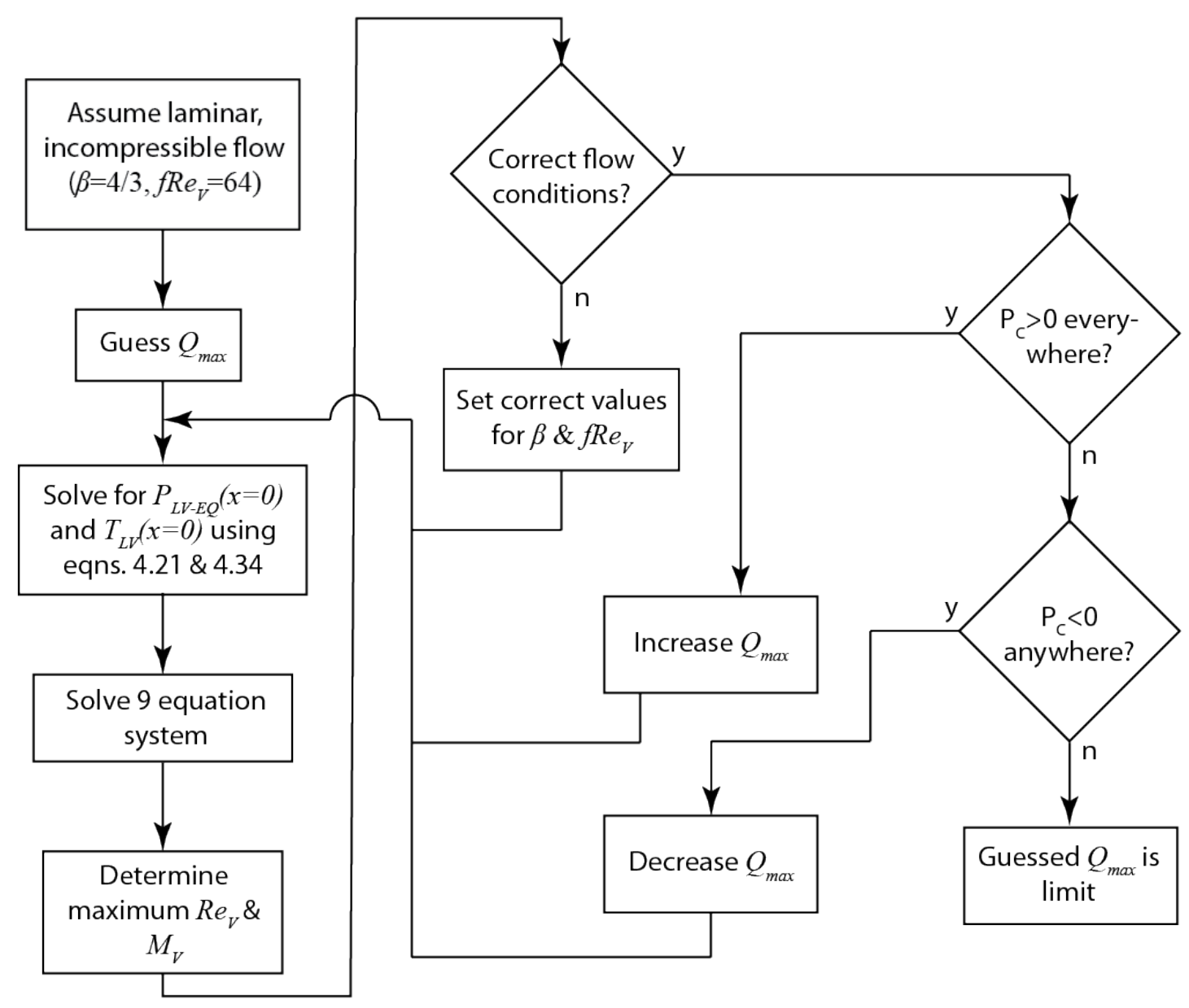

Figure 4.12. Flow diagram for the solution algorithm.

\section{3(e) Results and discussion}

The model is validated using the stainless steel (SS)304, sodium-charged heat pipe tested by Vinz and Busse [104]. An induction radio frequency heating system was used at 
the evaporator end of the heat pipe and a water-based flow-through, helium gas gap calorimeter at the condenser end for removing heat. The heat limit for a given temperature $T_{V}$ (measured at the wall in the adiabatic section) was found by decreasing the condenser temperature (by adjusting the calorimeter's flow) until a temperature spike was observed on the evaporator wall. The maximum transported heat flux was then determined from the flow rate and temperature rise of the calorimeter fluid. Relevant parameters for the heat pipe and setup are listed in Table 4.2. The temperature-dependent thermal conductivity of SS304 employed by the model is given in Appendix A. The thermophysical properties of the sodium working fluid are provided in Appendix B. The accommodation coefficient for sodium was taken as $c=1$, in agreement with experimental work that has been done on other liquid metals, including the alkalis [121]. 
Evaporation zone length, $L_{e} \quad 0.110 \mathrm{~m}$

Adiabatic zone length, $L_{a} \quad 0.160 \mathrm{~m}$

Condensation zone length, $L_{c} \quad 0.200 \mathrm{~m}$

Working Fluid (See Appendix B for thermo-physical property data)

Working fluid type Sodium

Case Design (See Appendix A for material property data)

Case material Stainless Steel AISI 304L

Outer wall radius, $R_{\text {wall }} \quad 6.0 \mathrm{~mm}$

Wall thickness, $b_{\text {wall }} \quad 1.5 \mathrm{~mm}$

Wick Design (See Appendix A for material property data)

Wick material Stainless Steel AISI 304L

Wick thickness, $b_{\text {wick }} \quad 0.15 \mathrm{~mm}$

Wick permeability $\quad 1.73 \times 10-8 \mathrm{~m} 2$

Pore radius, $R_{p} \quad 5.5 \mu \mathrm{m}$

\section{3(f) Solved variables with $F=1.0$}

Figure 4.13 shows the solved vapor pressure $P_{V}$, liquid pressure $P_{L}$, and equilibrium vapor pressure at the curved interface $P_{L V-E Q}$ as a function of axial position for the Vinz and Busse heat pipe operating at $T_{V, o}=927^{\circ} \mathrm{C}$ and with the active evaporator fraction set to $F=1.0$. 


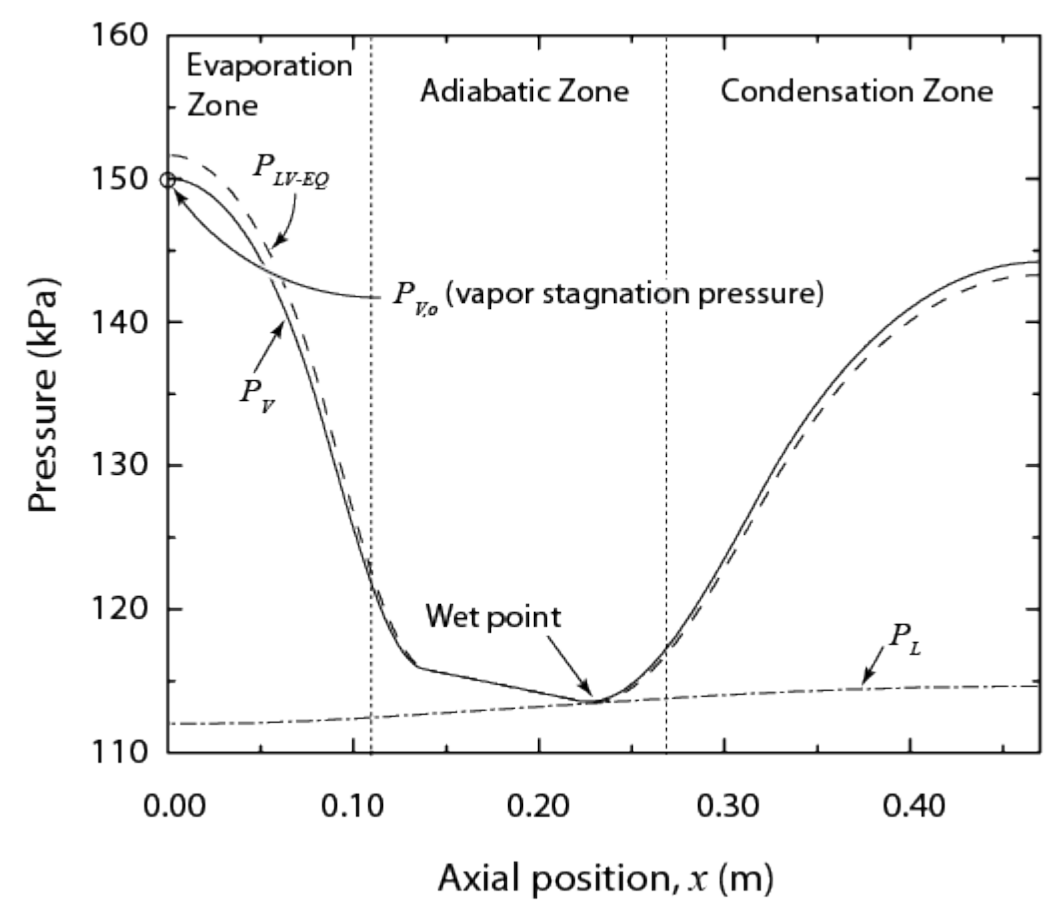

Figure 4.13. Calculated vapor and liquid pressure drops using the modified Chi model for the sodium heat pipe described by and operating at $Q_{\max }=21.6 \mathrm{~kW}$ with $T_{V, o}=927^{\circ}$.

The capillary pressure is zero at $x \approx 0.23 \mathrm{~m}$ and $P_{C}>0$ everywhere else, indicating that the solution algorithm has converged upon the capillary limit, $Q_{M A X}=21.6 \mathrm{~kW}$. Over the first $0.11 \mathrm{~m}$, heat enters the pipe and $P_{L V-E Q} / T_{L V}^{1 / 2}>P_{V} / T_{V}^{1 / 2}$ which is consistent with evaporation. The relationship is reversed $\left(P_{V} / T_{V}^{1 / 2}>P_{L V-E Q} / T_{L V}{ }^{1 / 2}\right)$ and consistent with condensation over the condenser length. Over the adiabatic section, $P_{L V-E Q} / T_{L V}^{1 / 2}=P_{V} / T_{V}^{1 / 2}$ where there is no thermodynamic driving force for a net change of phase. The vapor pressure $P_{V}$ can be seen to decrease in the evaporation zone and then recover in the condensation zone minus losses due to friction. One would expect then 
an acceleration and deceleration of the flow commensurate with this pressure profile, shown in Figure 4.14. The vapor Mach number remains far below unity indicating that the sonic limit has not been reached. The plot also shows that the Mach number experiences a small increase over the adiabatic portion of the pipe: the velocity is constant in this region and the Mach number increase is due to a decreasing vapor density, shown in Figure 4.15, over the same length.

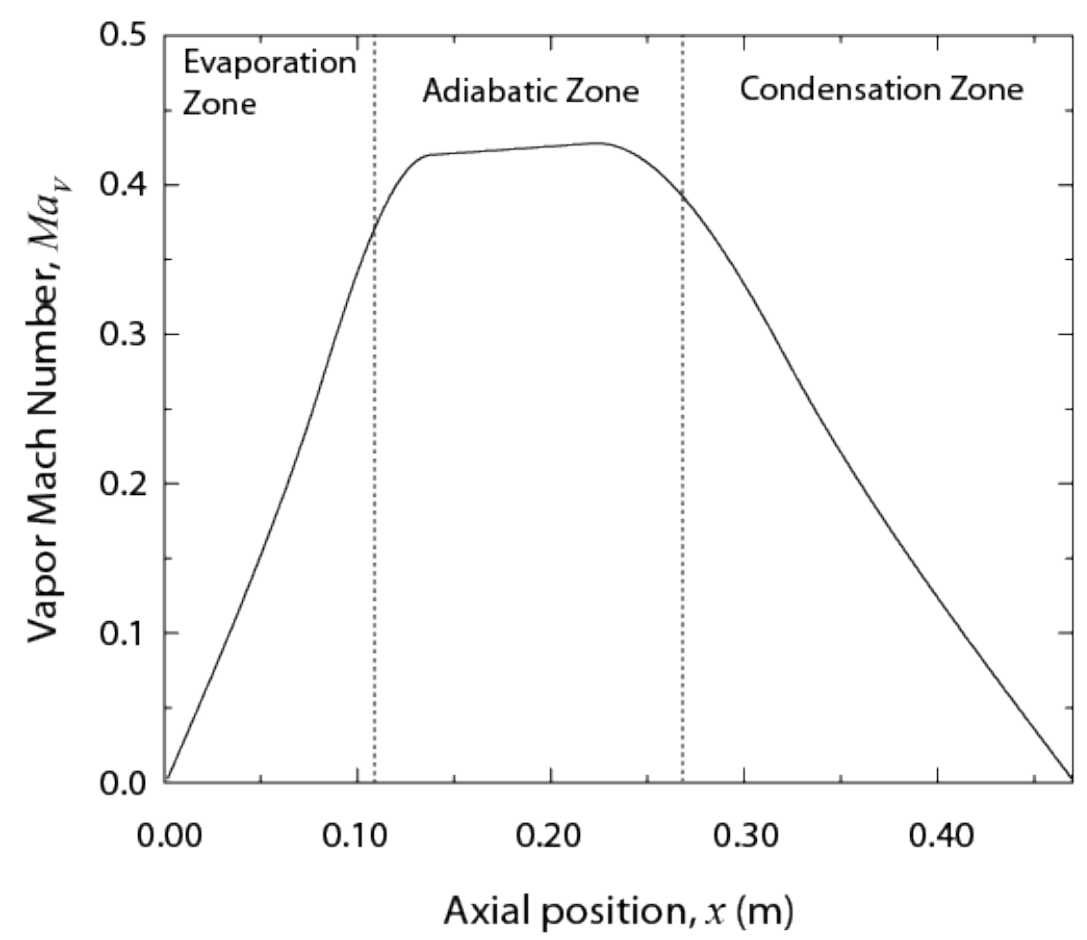

Figure 4.14. The vapor mach number as a function of position using the modified Chi model for the sodium heat pipe operating at $Q_{\max }=21.6 \mathrm{~kW}\left(T_{V, o}=927^{\circ} \mathrm{C}\right)$. 


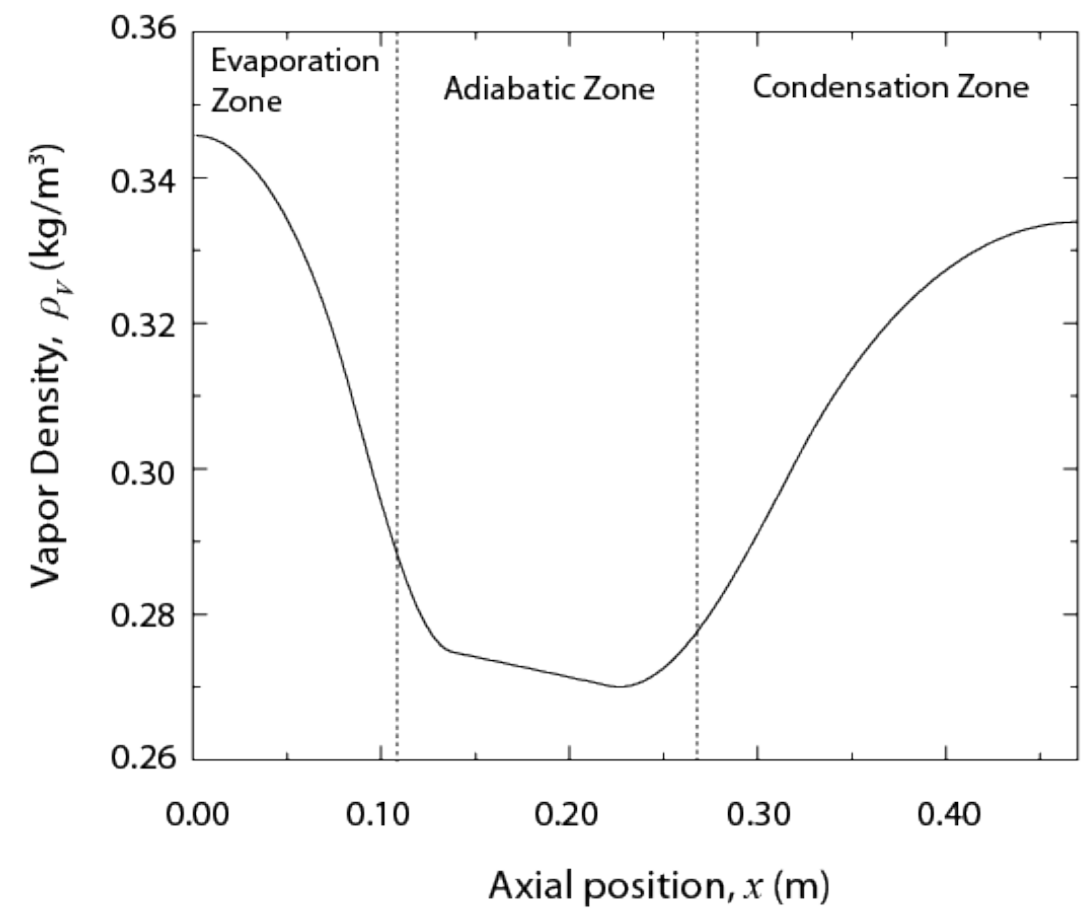

Figure 4.15. The vapor density as a function of position using the modified Chi model for the sodium heat pipe operating at $Q_{\max }=21.6 \mathrm{~kW}\left(T_{V, o}=927^{\circ} \mathrm{C}\right)$.

Figure 4.16 plots the meniscus radius as a function of position. The meniscus radius is at a minimum at the evaporator end cap, increases along the length of the heat pipe and is asymptotic to the wet point (as $P_{C} \rightarrow 0, \quad R_{M} \rightarrow \infty$ ), consistent with other high temperature heat pipe models [72], [73]. The corresponding meniscus profile is sketched above the plot. 


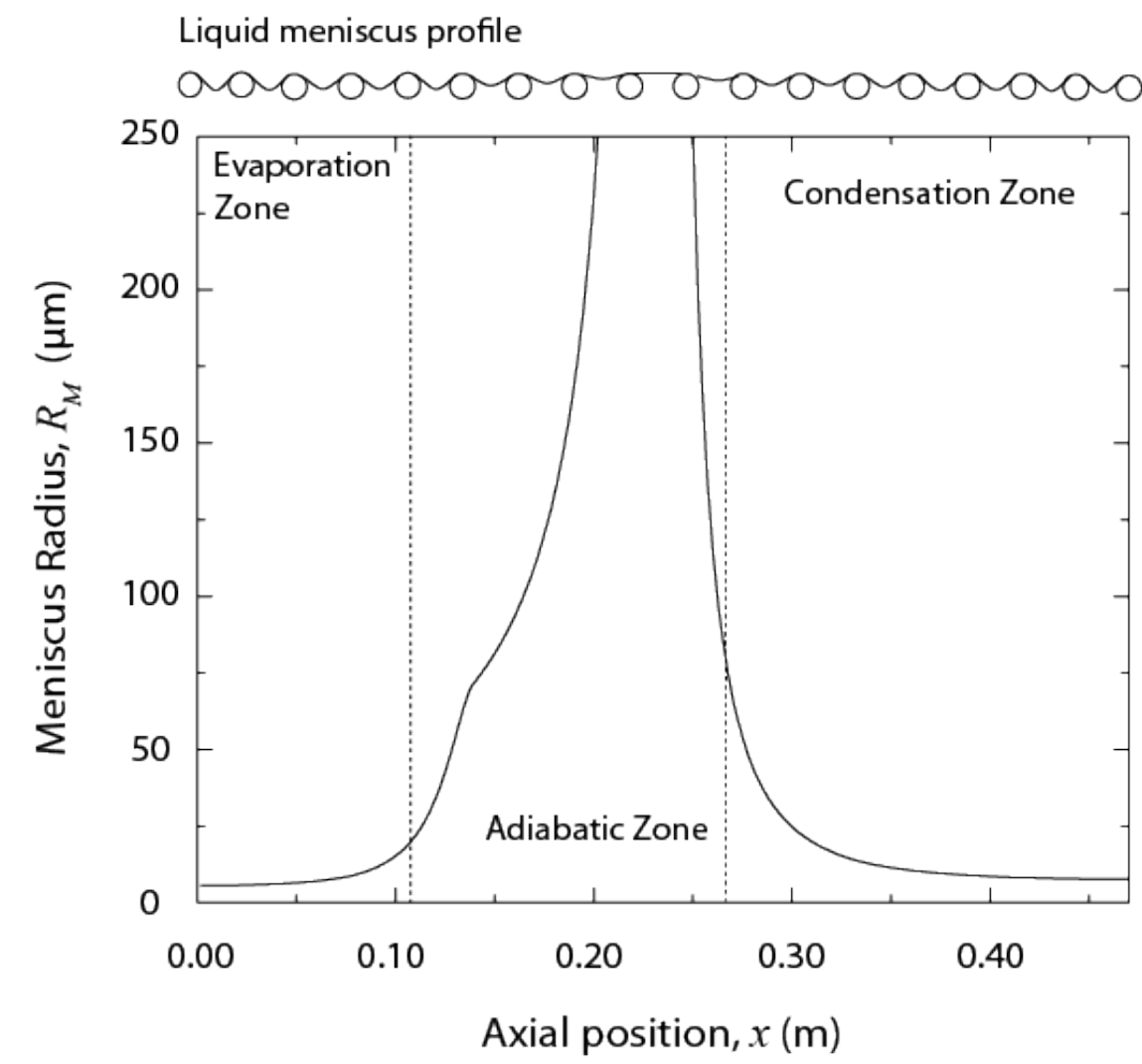

Figure 4.16. The meniscus radius as a function of position using the modified Chi model for the sodium heat pipe operating at $Q_{\max }=21.6 \mathrm{~kW}\left(T_{V, o}=927^{\circ} \mathrm{C}\right)$. A sketch of the liquid surface profile along the pipe is provided above the plot.

Figure 4.17 plots the temperature at the wall $\left(T_{W}\right)$, wall-liquid interface $\left(T_{W L}\right)$, liquid-vapor interface $\left(T_{L V-E Q}\right)$, and in the vapor $\left(T_{V}\right)$. Thermal gradients have been established through the thickness of the wall and wick to drive the heat by thermal conduction. Axial thermal gradients along the wall and wall-liquid interface are large, with a wall temperature difference of $\Delta T_{W} \approx 600^{\circ} \mathrm{C}$ between the evaporator and condenser end caps. However, the vapor temperature remains nearly isothermal. The interface superheat $\left(T_{L V}-T_{V}\right)$ remains less than $\pm 3.2^{\circ} \mathrm{C}$ over the pipe length. 


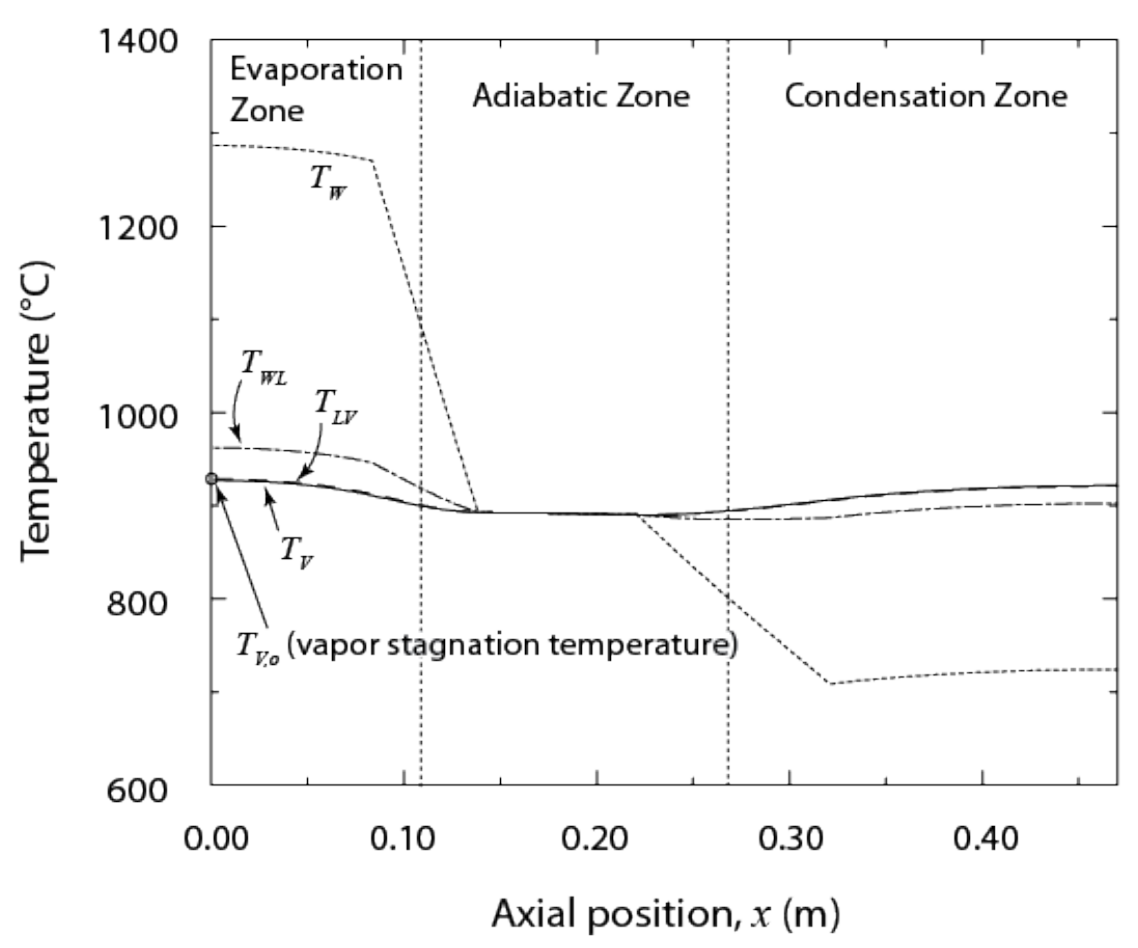

Figure 4.17. Temperature profiles generated by the modified Chi model for the sodium heat pipe operating at $Q_{\max }=21.6 \mathrm{~kW}\left(T_{V, o}=927^{\circ} \mathrm{C}\right)$.

\section{3(g) Influence of $F$ value}

Thus far, the results have assumed an active evaporation/condensation areal fraction of $F=1.0$. Figure 4.18 shows how the pressure drop changes with $10 \%$ of the unit cell area active in evaporation (i.e. $F=0.1$ ). The smaller active region amplifies the magnitude of $\frac{P_{L V-E Q}}{T_{L V}{ }^{1 / 2}}-\frac{P_{V}}{T_{V}{ }^{1 / 2}}$ along the heat pipe in order to elevate the evaporation/condensation flux. $P_{L V-E Q}$ is much more sensitive to increases in $\mathrm{Q}_{\max }$ with $F=0.1$ than at $F=1.0$. The result is a $17 \%$ reduction in the total heat which can be transported subject to the capillary limit $(21.6 \mathrm{~kW}$ at $F=1.0,17.9 \mathrm{~kW}$ at $F=0.1)$. Further down the heat pipe, $P_{L V-E Q}$ is 
depressed to balance the vapor condensation rate, having the effect of shifting the wet point well into the condenser.

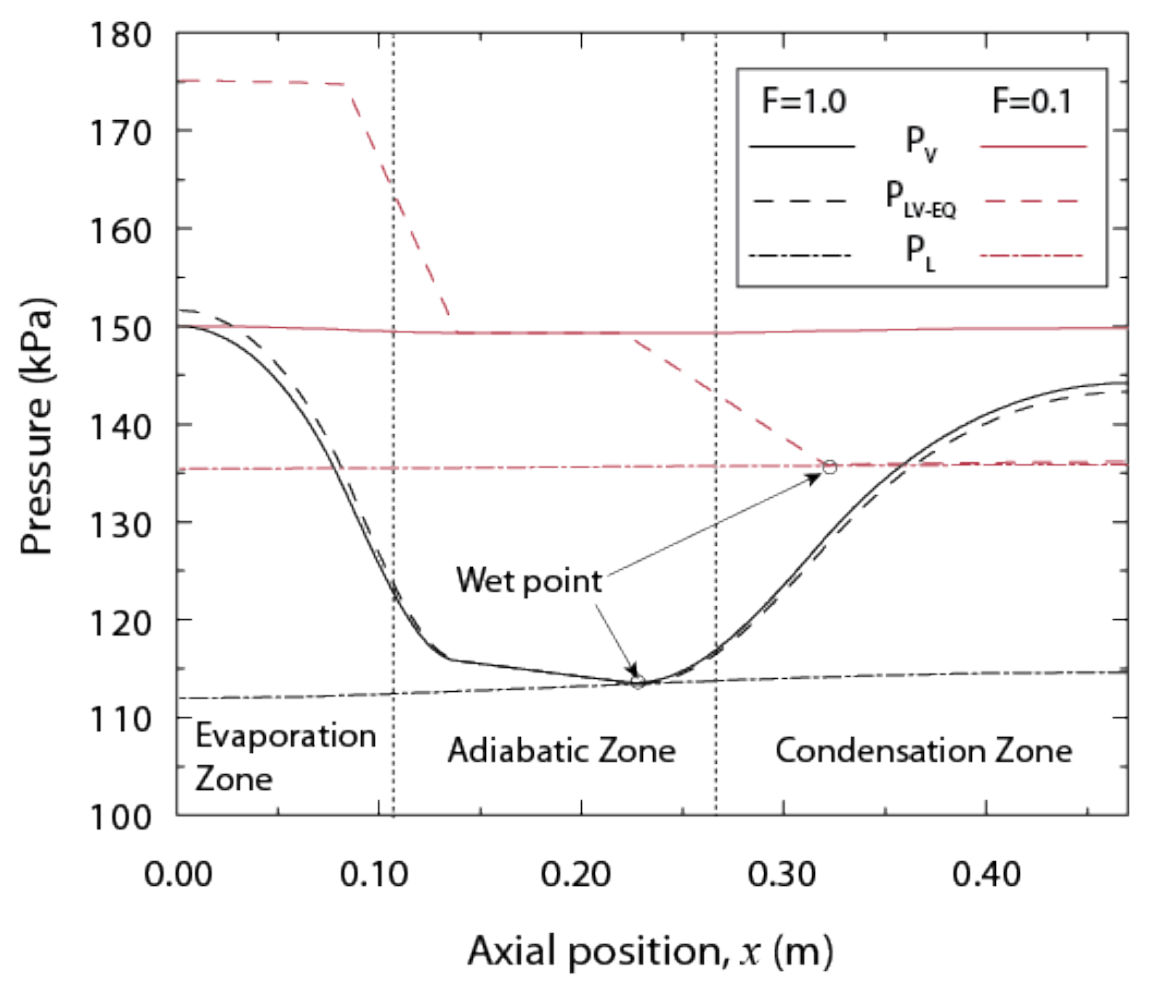

Figure 4.18. Comparison of the pressure drops for the modified Chi model using active interfacial mass transfer regions of $100 \%$ and $10 \%$ (i.e., $F=1.0$ and $F=0.1$, respectively). The wet point has shifted towards the middle of the condensation zone for the lower value of $F$.

Figure 4.19 compares the axial wall temperature profiles for $F=1.0,0.1$, and 0.01 at the capillary limit. The lower heat transport rates at smaller $F$ require smaller throughthickness temperature gradients for heat conduction through the pipe wall. At $F=1.0$, the maximum wall temperature reaches nearly $1300^{\circ} \mathrm{C}$, approaching the incipient melting temperature of most stainless steels [122]. Operating a sodium-charged stainless steel heat 
pipe near these temperatures is likely unsafe and above any reasonable factor of safety. But because the Chi model does not account for thermal gradients through the case wall, this operating condition would be treated as a flow limit - not a material one - and accepted as the upper bound of performance.

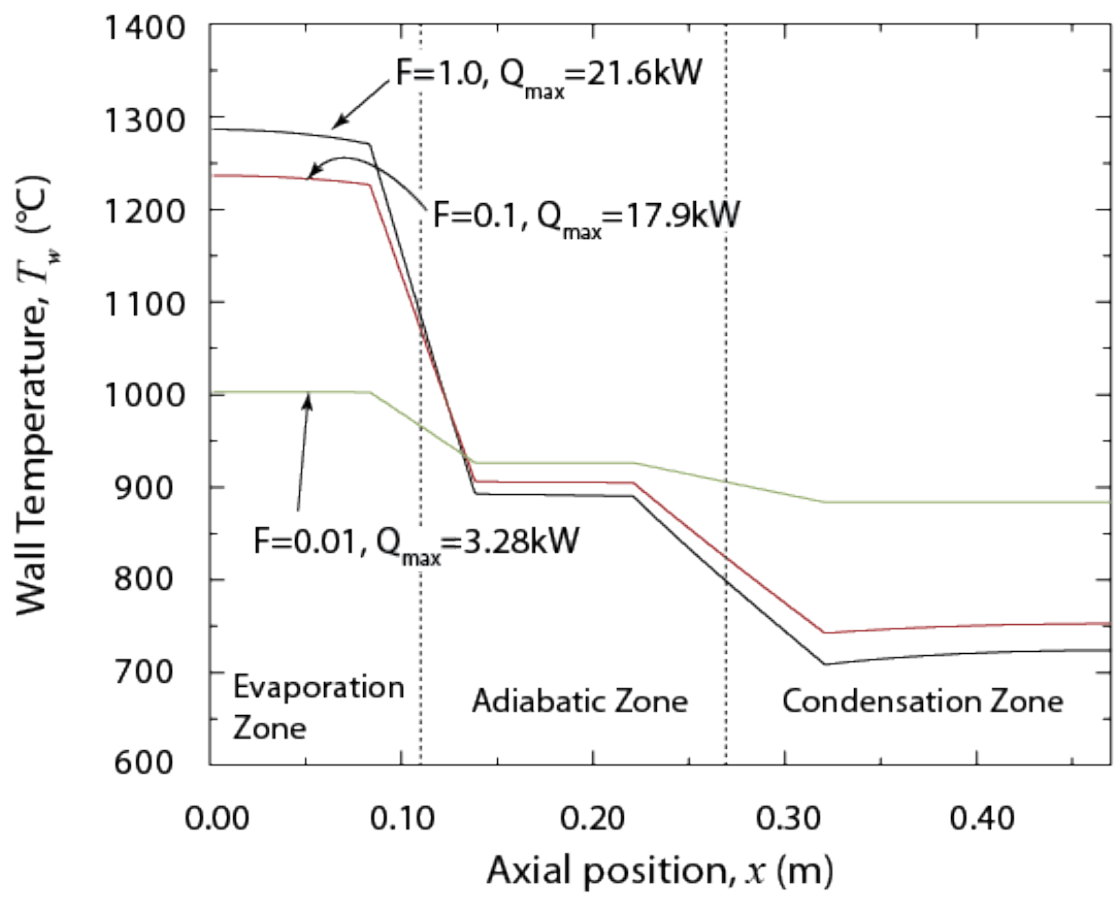

Figure 4.19. Axial wall temperature profile, $T_{W}$, for several $F$ values.

\section{3(h) Validation of the Modified Chi Model}

Figure 4.20 shows a performance map for the Vinz and Busse heat pipe [104]. The viscous and sonic limits have been reproduced from that work, along with their experimental findings. Each data symbol represents changes or improvements to their experimental setup in an attempt to improve heat pipe performance. The sonic and viscous models show good 
agreement with the data, following the trend right up until the capillary limit is the expected failure mechanism. The Chi model predicts the limit to be approximately four times higher than what was observed. The modified Chi model with $F=1.0$, while slightly lower than the traditional Chi model, still over predicts the limit. The model is seen to closely predict the observed limits with $F=0.05$.

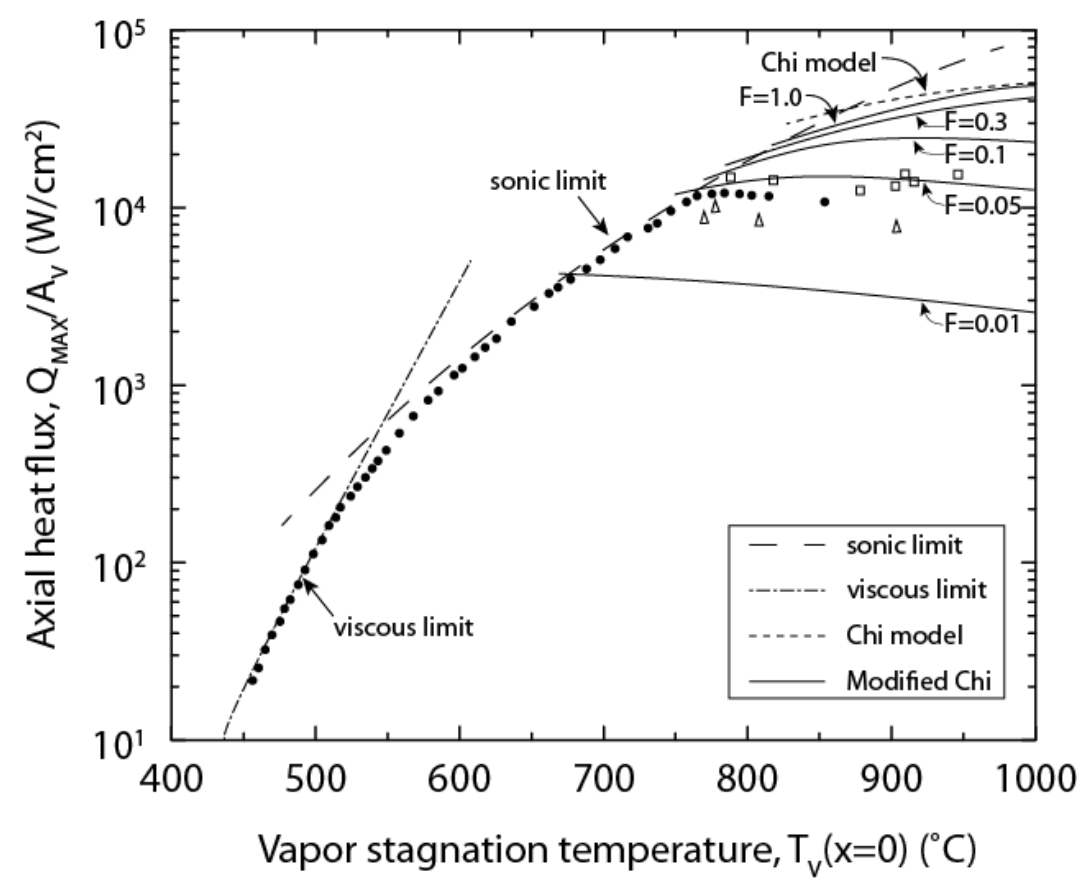

Figure 4.20. Limits map for heat pipe performance. Experimental data, viscous, and sonic limits reproduced from [104]. Each data symbol type represents changes made to the experimental setup to try and improve the experimentally determined limits in the capillary limit region.

In order to justify the areal fraction, we approximate the wire mesh wick used by Vinz and Busse [104] as having wires of diameter $d_{w}$ and seperation distance $2 R_{P}$, shown in Figure 4.21. The unit cell area is; 


$$
A_{C}=\left(d_{w}+2 R_{P}\right)^{2}
$$

As described earlier, the portion of this area which participates in evaporation is much smaller. For a completely wetting fluid, we assume the liquid surface over the wires is the non-evaporating, ultra-thin film region, leaving only the thin film and intrinsic meniscus regions of the pores to participate in evaporation heat transfer. This assumption puts a limit on the maximum areal fraction for evaporation, given by;

$$
F_{\text {max }}=\frac{4 R_{P}{ }^{2}}{\left(d_{w}+2 R_{P}\right)^{2}}
$$

Equation (4.43) states that the theoretical limit of $F=1.0$ can not be met; evaporation will always occur over only a portion of a wick's surface. Wicks which have large wire diameters relative to their pore size will have smaller active evaporation areas.

On a per unit cell basis, the evaporating area can be expressed in terms of the shoulder of the evaporating film, $w$;

$$
A_{A}=8 R_{P} w-4 w^{2}
$$

The shoulder $w$ can be expressed as a function of the evaporating film length, $k$, taken from the tangent point of the meniscus as shown;

$$
w=R_{P}-\sqrt{R_{P}{ }^{2}-\kappa^{2}}
$$


which, when substituted into equation (4.44), provides an expression for the active evaporation area as a function of the evaporating film length;

$$
A_{A}=4 \kappa^{2}
$$

Using equations (4.31), (4.42), and (4.44), the active areal fraction for a screen wick is;

$$
F=\frac{A_{A}}{A_{C}}=\frac{4 \kappa^{2}}{\left(d_{w}+2 R_{P}\right)^{2}}
$$

which can be solved for the active thin film length;

$$
\kappa=\frac{d_{w}+2 R_{P}}{2} \sqrt{F}
$$

For a pore radius of $R_{P}=5.5 \mu \mathrm{m}$ wire diameter of $d_{w}=20 \mu \mathrm{m}$ and an active areal fraction of $F=0.05$, an evaporating film length of $\kappa=3.5 \mu \mathrm{m}$ is calculated. Monazami [123] investigated, by numerical analysis, the evaporation from a fully-wetted meniscus inside a microchannel due to heating through the walls. Analysis of microchannel widths from 5 to $100 \mu \mathrm{m}$ showed that a $1 \mathrm{~K}$ superheat produced maximum capillary pressures of approximately $50 \mathrm{kPa}$, close to the maximum values of $40 \mathrm{kPa}$ computed in this work. The plot shown in Figure 4.22, reproduced from [123], shows the evaporation flux as a function of thin film length at a $1 \mathrm{~K}$ superheat for various microchannel sizes. Nearly all the evaporation occurs over the first 2 to $4 \mu \mathrm{m}$ of the thin film which agrees well with the value calculated here for $F=0.05$. 


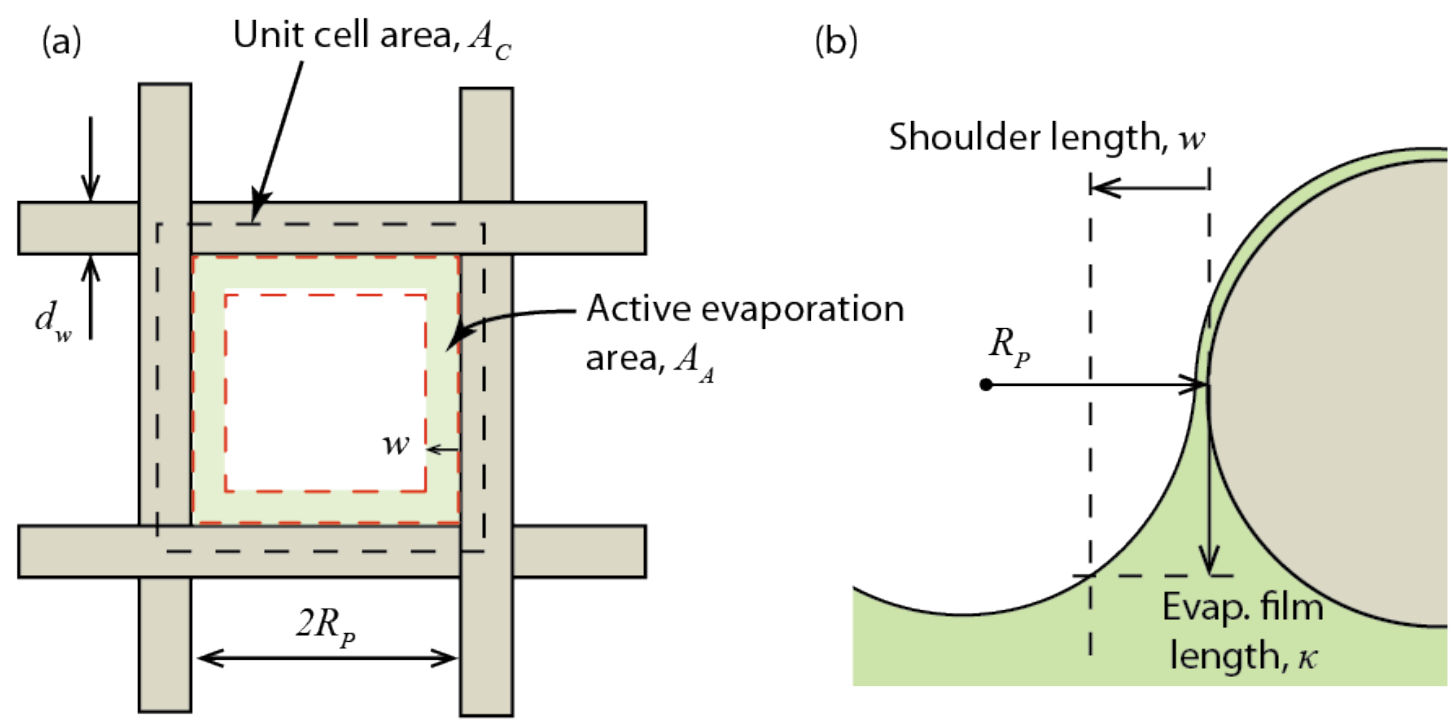

Figure 4.21. Geometry of the wetted unit cell: (a) elevated view, (b) profile view.

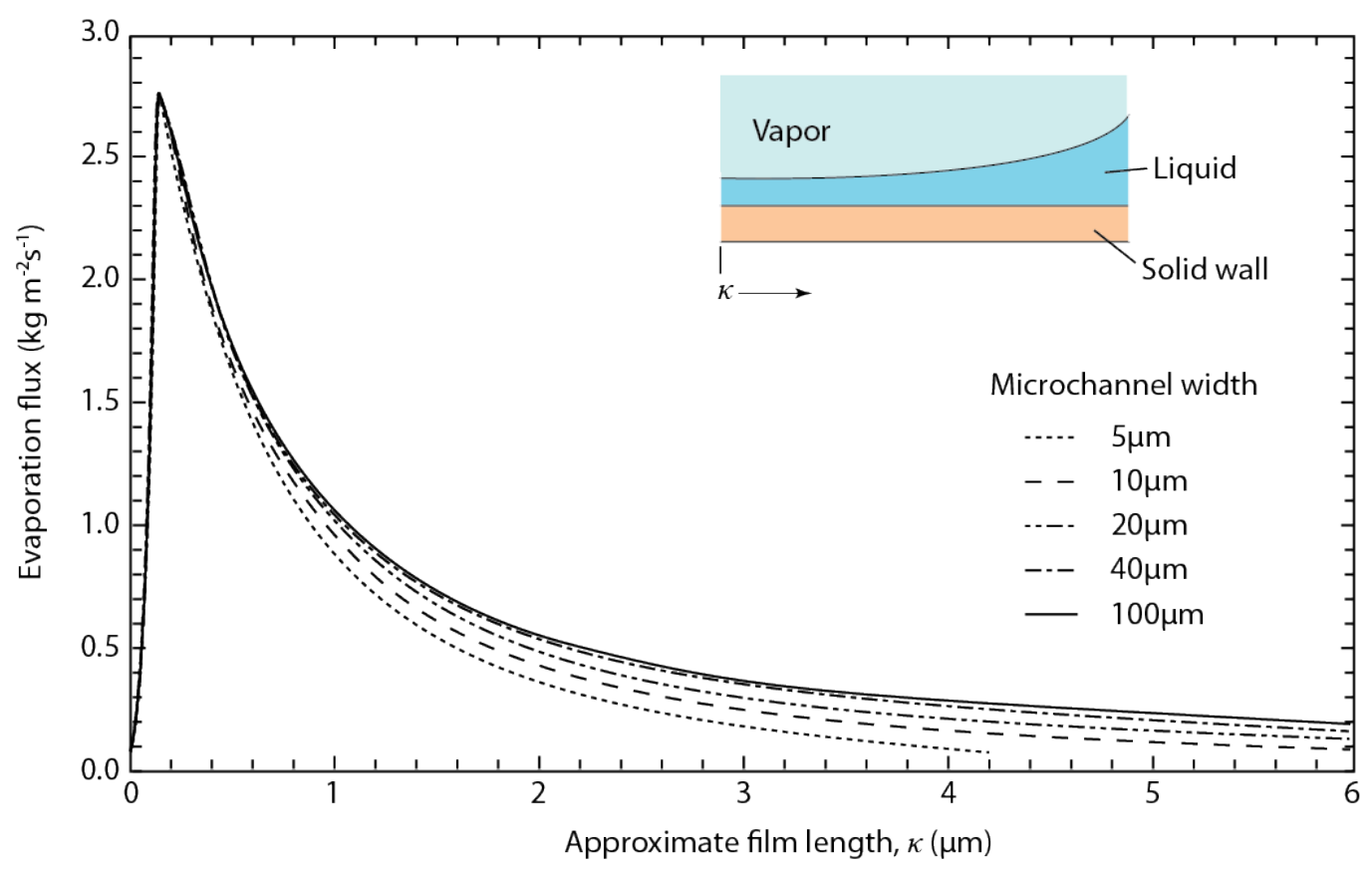

Figure 4.22. Evaporation mass flux as a function of distance along a microchannel wall of varying widths and with a wall superheat of $1 K$. The evaporating film length is nearly independent of microchannel width. Significant evaporation occurs within the first 2 to $4 \mu \mathrm{m}$. Reproduced from [123]. 


\subsection{Modified Chi Model (Wedge Geometry)}

The modified Chi model was derived for a cylindrical heat pipe and shown to predict the capillary limit with improved performance over the traditional capillary limit model for high temperature heat pipes. It is now adapted from the cylindrical geometry to that of a wedge-shaped leading edge. As before, the vapor flow is based on compressible flow theory and makes the following assumptions:

(i) Fully developed flow: Boundary layer separation occurs in diverging ducts with sufficiently large half angles and flow velocities [124]. For the small half angle explored $\left(\varphi=6^{\circ}\right)$, we assume no boundary separation.

(ii) Constant velocity profiles: The velocity distribution across the vapor core crosssection is approximated by a constant velocity profile, so that the velocity is taken to be an average velocity.

(iii) No slip upstream and downstream: At the upstream end of the evaporator and the downstream end of the condenser, no slip conditions are assumed and stagnation conditions persist at these points. 
(iv) Normal injection velocity: The direction of the velocity vector for the injected/suctioned mass is perpendicular to the flow direction, and therefore contributes no momentum in the direction of the vapor flow.

Figure 4.23 depicts the system geometry and boundary conditions used to model the vapor and liquid flows and heat transfer across the wick and wall as they pertain to the capillary limit. The leading edge tip of radius $R_{L E}$ is modeled as a diverging duct of total length $L_{t}$ given by;

$$
L_{t}=L_{e}+L_{c}
$$

where the evaporator and condenser lengths are defined as;

$$
\begin{gathered}
L_{e}=R_{L E}\left(\frac{\pi}{2}-\varphi\right) \cos \varphi \\
L_{c}=L_{\text {flat }} \cos \varphi
\end{gathered}
$$

Unlike the cylindrical system, there is no adiabatic section. This is a consequence of the heat transfer profile from the external hypersonic flow.

The diverging duct height, $H_{V}$, is a function of position along the x-axis. An expression for the height as a function of axial position $x$ is easily derived; 


$$
\begin{aligned}
H_{V}(x)=R_{L E} & {\left[\cos \varphi-\left(\frac{\pi}{2}-\varphi\right) \sin \varphi\right]-\left(b_{\text {wall }}+b_{\text {wick }}\right) \sec \varphi } \\
+ & x \tan \varphi
\end{aligned}
$$

The vapor space cross-sectional area is then;

$$
A_{V}(x)=2 W H_{V}(x)
$$

where $W$ is the wedge width. 
(a) Real space

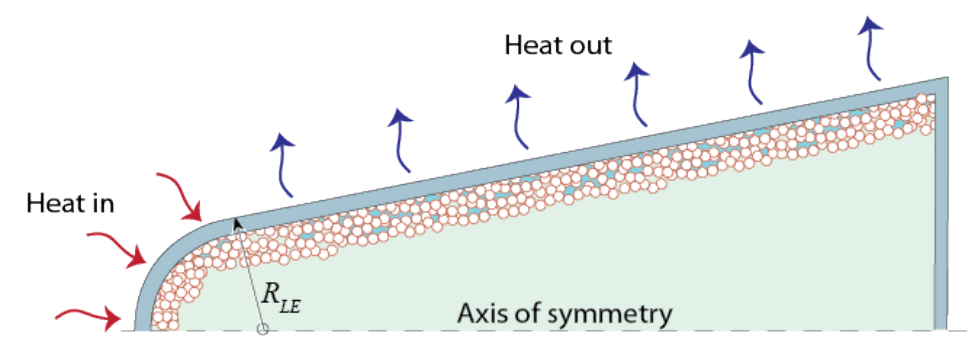

(b) Computational space

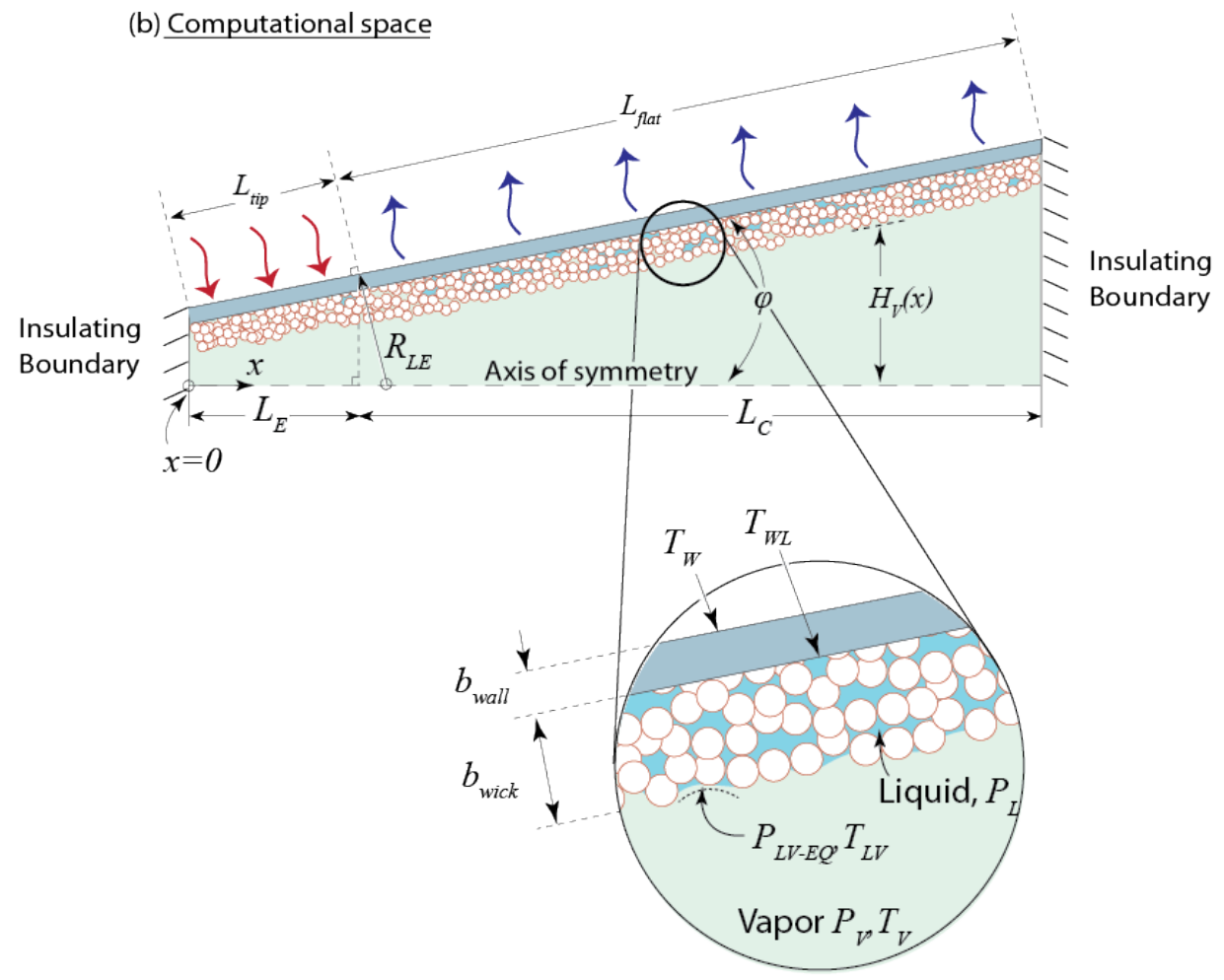

Figure 4.23. (a) Leading edge thermal spreader with wedge geometry. The real system in (a) is modeled as the diverging duct in (b).

The fundamental principles of the modified Chi model is identical for the cylindrical and leading edge geometry. The Laplace equation (equation (4.16)), liquid pressure drop equation (equation (4.17)), Kelvin equation (equation (4.21)), perfect gas law (equation 
(4.35)), and Clausius-Clapeyron equation (equation (4.36)) remain identical. The gravitational pressure drop term in the Laplace equation is replaced by $\Delta P_{G} \approx$ $2 \rho_{L} g H_{V}\left(x=L_{E}\right) \sin \varphi$ for the wedge. However, due to geometry differences - most notably the diverging duct design - the vapor momentum equation, wall/wick heat balance, wick/vapor heat balance, and Schrage equation require the following modifications.

\section{4(a) Vapor Equation}

The axial vapor pressure drop for the diverging wedge is given by (the complete derivation is given in Appendix D);

$$
\begin{gathered}
d P_{V}=-\frac{\beta}{\rho_{V} A_{V}^{2} \lambda^{2}}\left(2 Q d Q-\frac{Q^{2}}{\rho_{V}} d \rho_{V}-\frac{Q^{2}}{A_{V}} d A_{V}\right) \\
-\frac{2 \mu_{V} f \operatorname{R} e_{V}\left(W+2 H_{V}\right)^{2} \cos \varphi}{\lambda \rho_{V} A_{V}{ }^{3}} Q d x
\end{gathered}
$$

The $d Q$ term accounts for changes in pressure from the rise and fall of the vapor mass flow rate as the input power is injected and withdrawn over the wedge length. The $d \rho_{V}$ handles vapor compressibility and the $d A_{V}$ term increases the pressure as the vapor space height increases down the length of the wedge. The $d x$ term accounts for non-recoverable viscous losses. As with the cylindrical system, a forward difference discretization scheme was used to pose the vapor equation into an algebraic form for solving. 


\section{3(b) Wall/Wick and Wick/Vapor Heat Balance}

Assuming thin walls, the through-thickness wall and wick heat fluxes is given by the linear heat conduction equation;

$$
\begin{gathered}
q_{w}=k_{\text {wall }} \frac{T_{W L}-T_{W}}{b_{\text {wall }}} \\
q_{\text {wick }}=k_{\text {wick }} \frac{T_{L V}-T_{W L}}{b_{w i c k}}
\end{gathered}
$$

At steady state, these fluxes must balance at the wall/wick interface. Setting equations (4.55) and (4.56) equal provides an expression for the liquid-vapor interface temperature, $T_{L V}$;

$$
T_{L V}=\left(T_{W L}-T_{W}\right)\left(\frac{k_{\text {wall }} b_{\text {wick }}}{k_{\text {wick }} b_{\text {wall }}}\right)+T_{W L}
$$

The average phase change (evaporation/condensation) heat flux at the liquid/vapor interface is given by;

$$
q_{P h C}=\lambda \bar{m}_{i}
$$

For evaporation, this must balance the heat driven to the interface through the wick and, for condensation, the heat driven from the interface through the wick to the case wall. Equating equations (4.56) and (4.58), and noting that $q_{P h C}=\frac{1}{2 W} \frac{d Q}{d x}$, an expression for the temperature at the wall/liquid interface is found; 


$$
T_{W L}=\frac{b_{w i c k}}{2 W k_{w i c k}} \frac{d Q}{d x}+T_{L V}
$$

\section{3(c) Schrage Equation}

The average evaporation/condensation mass flux $\bar{m}_{i}$ is related to the vapor heat flow gradient $\frac{d \mathrm{Q}}{d x}$ by;

$$
\bar{m}_{i}=\frac{1}{2 W \lambda} \frac{d \mathrm{Q}}{d x}
$$

Using equations (4.32), (4.33), and (4.60), an expression for the vapor heat flow gradient as a function of the interfacial and vapor pressures and temperatures is obtained;

$$
\frac{d Q}{d x}=\frac{4 c}{2-c}\left(\frac{M}{2 \pi R}\right)^{1 / 2}\left(\frac{P_{L V-E Q}}{T_{L V}{ }^{1 / 2}}-\frac{P_{V}}{T_{V}{ }^{1 / 2}}\right) W \lambda F
$$

\section{$\underline{4.3(d) \text { Boundary Conditions }}$}

As demonstrated in Chapter 3, under hypersonic flight conditions, nearly all of the heat is acquired along the tip curvature and there is a steady decrease in the stored heat over the length of the flat surface. Three different models were used to "stitch" this heat transfer profile together over the surface of the wedge. For the current limits model, the heat flux 
impinging the wall is approximated using a hyperbolic tangent function which simplifies the model boundary condition and has the added benefit of being continuous over the length;

$$
q_{g w}(x)=\frac{1}{2}\left[q_{g w, s t}-q_{m i n}\right]\left[1-\tanh \left(x C_{1}-L_{E} C_{2}\right)\right]+q_{m i n}
$$

such that the wall heat flux (introduced at equation (3.1) as $q_{w}=q_{g w}+q_{r}$ ) becomes;

$$
q_{w}(x)=\frac{1}{2}\left[q_{g w, s t}-q_{m i n}\right]\left[1-\tanh \left(x C_{1}-L_{E} C_{2}\right)\right]+q_{m i n}+q_{r}
$$

We approximate radiation cooling as $q_{r}=-\sigma \varepsilon T_{V, o}{ }^{4} \cdot q_{g w, s t}$ is the maximum heat flux which impinges the stagnation point and serves as a model input. Equation (4.63) with its unknown constants $q_{m i n}, C_{1}$, and $C_{2}$, and its integral, $Q(x)=2 W \int q_{w}(x) d x$ (and its integration constant) can be solved to obtain the wall heat flux profile using the following boundary conditions;

$$
\begin{gathered}
q_{w}(x=0)=q_{g w, s t}+q_{r} \\
q_{w}\left(x=L_{E}\right)=0 \\
Q(x=0)=0 \\
Q\left(x=L_{T}\right)=0
\end{gathered}
$$

Boundary condition (4.64)(b) assumes the net heat flux is zero at the transition between the curved region and flat surface. Figure 4.24(a) shows that the tanh approximation compares 
well with that of the hypersonic theory presented in Chapter 3 for a Mach 6 IN718 system operating with an isothermal wall temperature of $T_{W}=806^{\circ} \mathrm{C}$. The value for $q_{w}$ at the stagnation point is of course identical by virtue of how the approximation has been constructed. As before, $Q(x)$ is the cumulative power in the system. The opposing ends are assumed adiabatic so that all heat must enter and exit the top surface. Therefore, to satisfy a heat balance, $Q(x)$ must equal zero at the leading edge tip and at the far end of the condenser section, which is confirmed in Figure 4.24(b). As a result of our assumption above in (4.64)(b), the maximum cumulative power is found at $x=L_{E}$. At convergence, the capillary heat flux limit, $q_{w}(x=0)=q_{c a p}$. 

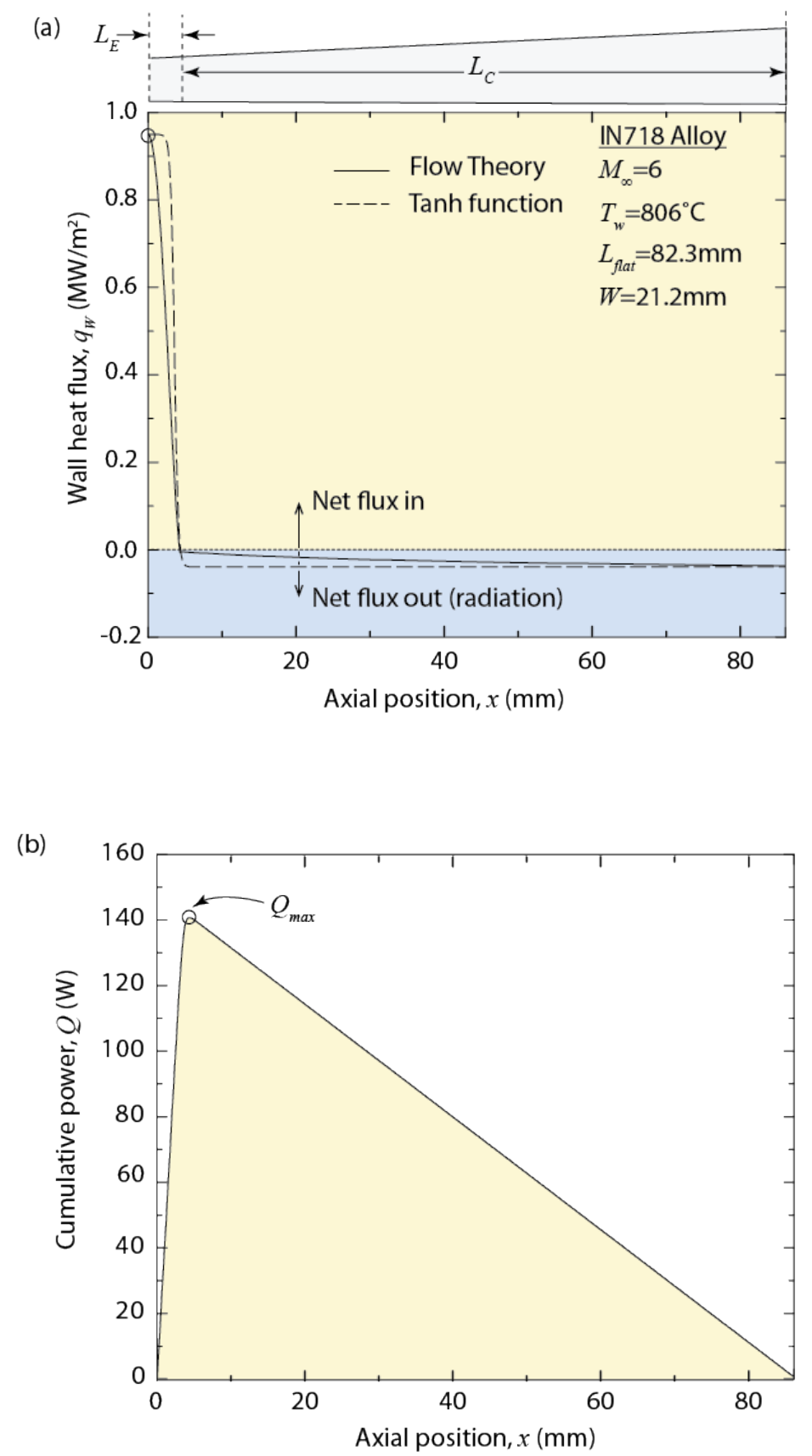

Figure 4.24. Heat profiles used as thermal boundary conditions in the model. In (a), the simplified tanh function for the heat flux through the wall compares well to the full hypersonic theory. The cumulative power of the system is shown to obey an energy balance using the tanh approximation in (b). $R_{L E}=3 \mathrm{~mm}$ and $\varphi=6^{\circ}$. 
4.3(e) Solution Method

The system of nine equations is summarized in Table 4.3. Wolfram Mathematica was again used to solve the system of equations using the identical algorithm for the cylindrical case, except that the laminar value for $f R e_{V}$ becomes $f R e_{V}=54$, which is for flow in wide passages [125]. The system considered was a sodium-charged IN718 alloy leading edge with a nickel foam wick. Geometric and material properties are given in Table 4.4 . 
Table 4.3. List of the system of nine equations and unknown variables for the leading edge heat pipe.

\begin{tabular}{|c|c|c|c|}
\hline Name & Eqn. No. & Equation & $\begin{array}{l}\text { Unknown } \\
\text { Variable }\end{array}$ \\
\hline Full Laplace & $(4.16)$ & $P_{C}(x)=P_{L V-E Q}(x)-P_{L V-E Q}(x=0)+\frac{2 \sigma_{L V}}{R_{P}}-\left.\Delta P_{L}\right|_{0} ^{x}-\Delta P_{G}$ & $P_{L V-E Q}$ \\
\hline Liquid pressure drop & $(4.17)$ & $\left.\Delta P_{L}\right|_{0} ^{x}=\frac{\mu_{L}}{\rho_{L} \lambda A_{W} K} \int_{0}^{x} Q d x$ & $\Delta P_{L}$ \\
\hline Kelvin equation & $(4.21)$ & $P_{L V-E Q}=P_{V, R E F} \times \operatorname{Exp}\left[\frac{M\left(\lambda \rho_{L}\left(T_{L V}-T_{V, R E F}\right)-P_{C} T_{V, R E F}\right)}{\rho_{L} R T_{L V} T_{V, R E F}}\right]$ & $P_{C}$ \\
\hline Wall/Wick heat balance & $(4.57)$ & $T_{L V}=\left(T_{W L}-T_{W}\right)\left(\frac{k_{\text {wall }} b_{\text {wick }}}{k_{\text {wick }} b_{\text {wall }}}\right)+T_{W L}$ & $T_{L V}$ \\
\hline $\begin{array}{l}\text { Wick/Vapor heat } \\
\text { balance }\end{array}$ & $(4.59)$ & $T_{W L}=\frac{b_{w i c k}}{2 W k_{w i c k}} \frac{d Q}{d x}+T_{L V}$ & $T_{W}$ \\
\hline Schrage Equation & $(4.61)$ & $\frac{d Q}{d x}=\frac{4 c}{2-c}\left(\frac{M}{2 \pi R}\right)^{1 / 2}\left(\frac{P_{L V-E Q}}{T_{L V}^{1 / 2}}-\frac{P_{V}}{T_{V}^{1 / 2}}\right) W \lambda F$ & $T_{W L}$ \\
\hline Vapor momentum & $(4.54)$ & $\begin{array}{r}d P_{V}=-\frac{\beta}{\rho_{V} A_{V}{ }^{2} \lambda^{2}}\left(2 Q d Q-\frac{Q^{2}}{\rho_{V}} d \rho_{V}-\frac{Q^{2}}{A_{V}} d A_{V}\right) \\
-\frac{2 \mu_{V} f R e_{V}\left(W+2 H_{V}\right)^{2} \cos \varphi}{\lambda \rho_{V} A_{V}{ }^{3}} Q d x\end{array}$ & $P_{V}$ \\
\hline
\end{tabular}


Perfect Gas

$$
P_{V}=\frac{\rho_{V} R T_{V}}{M}
$$

$$
P_{V}=P_{V, R E F} \times \operatorname{Exp}\left[\frac{M \lambda}{R}\left(\frac{1}{T_{V, R E F}}-\frac{1}{T_{V}}\right)\right]
$$


Table 4.4. Relevant parameters for the sodium-IN718 Alloy leading edge system.

Leading edge radius, $R_{L E} \quad 3 \mathrm{~mm}$

Flat surface length, $L_{\text {flat }} \quad 82.3 \mathrm{~mm}$

Half angle, $\varphi \quad 6^{\circ}$

Working Fluid (See Appendix B for thermo-physical property data)

Working fluid type Sodium

Case Design (See Appendix A for thermo-physical property data)

Case material IN718

Wall thickness, $b_{\text {wall }}$

$1.59 \mathrm{~mm}$

Case width, $W$

$21.2 \mathrm{~mm}$

Wick Design

Wick material Stochastic open-cell nickel foam

Wick thickness, $b_{\text {wick }}$

$0.71 \mathrm{~mm}$

Wick permeability

$7.74 \times 10-9 \mathrm{~m} 2$

Pore radius, $R_{P}$

$225 \mu \mathrm{m}$

\section{3(f) Results and Discussion}

Figure 4.25 through Figure 4.28 show flow, pressure, and temperature profiles for the leading edge operating with vapor temperature $T_{V}(x=0)=827^{\circ} \mathrm{C}$ at the solved capillary limit of $q_{\text {cap }}=217.1 \mathrm{~kW} / \mathrm{m}^{2}$ with $F=0.05$.

The vapor Mach number shows a steep increase from the no-slip boundary condition $\left(M_{V}=0\right)$ imposed at the upstream end of the vapor space. The rise takes place along the evaporator with its peak corresponding to the location of $Q_{\max }$. Therefore, the points of 
maximum heat flow rate and maximum vapor velocity are consistent. The peak is well below the criteria $\left(\mathrm{M}_{\mathrm{V}} \geq 0.3\right)$ at which compressible effects become non-negligible. The steep rise in vapor velocity is caused by the high mass injection rate from evaporation. As vapor molecules condense out the stream over the flat portion, the vapor Mach number decreases. The decreasing Mach number over the condenser section is steeper than what would be seen for a constant area system because the diverging cross-sectional area contributes to the deceleration of the vapor velocity.

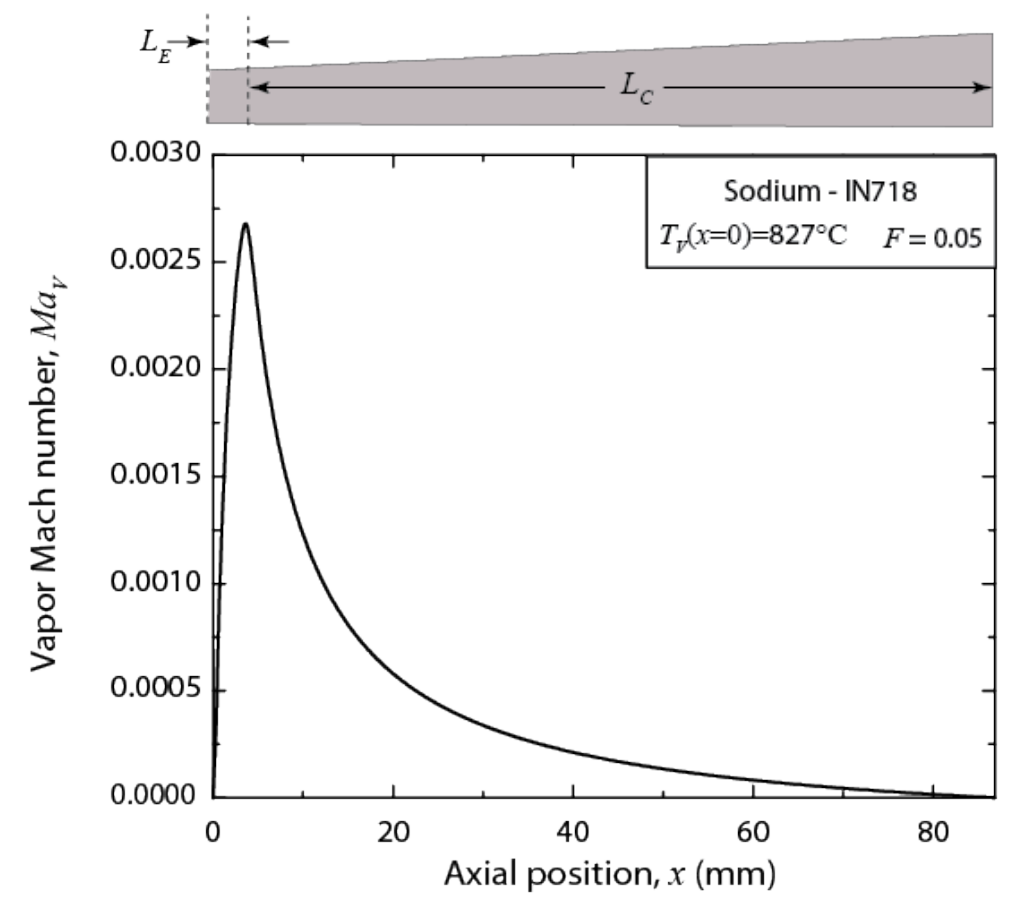

Figure 4.25. Vapor Mach number as a function of axial position for the Sodium-IN718 leading edge operating at its capillary limit of $q_{\text {cap }}=217.1 \mathrm{~kW} / \mathrm{m}^{2}$.

The axial pressure profiles are given in Figure 4.26. The capillary pressure reaches its maximum value $\left(2 \sigma_{L V} / R_{P}\right)$ at $x=0$. The pressure drop of $P_{L V-E Q}$ is substantially larger 
than $P_{V}$ over the curved tip, a critically important aspect which determines the capillary limit and one which would go unnoticed in the Chi model.

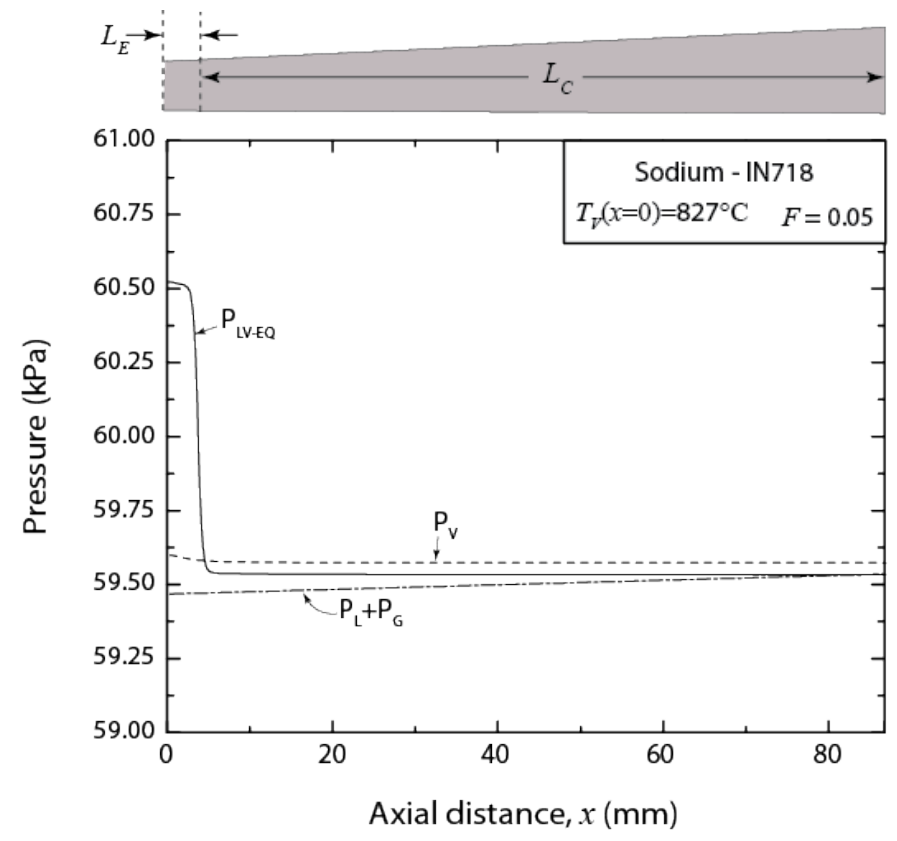

Figure 4.26. Pressure profiles as a function of axial position for the Sodium-IN718 leading edge operating at its capillary limit of $q_{\text {cap }}=217.1 \mathrm{~kW} / \mathrm{m}^{2}$

The variation in capillary pressure, $P_{C}$, and meniscus radius, $R_{M}$, are plotted as a function of position in Figure 4.27. $P_{C}$ is largest at minimum $R_{M}\left(=R_{P}\right)$ as stipulated by the Laplace equation. The wet point occurs at the downstream end of the leading edge where $P_{C}=0, R_{M} \rightarrow \infty$ (flat interface), and $P_{L V-E Q}=P_{L}+P_{G}$. 


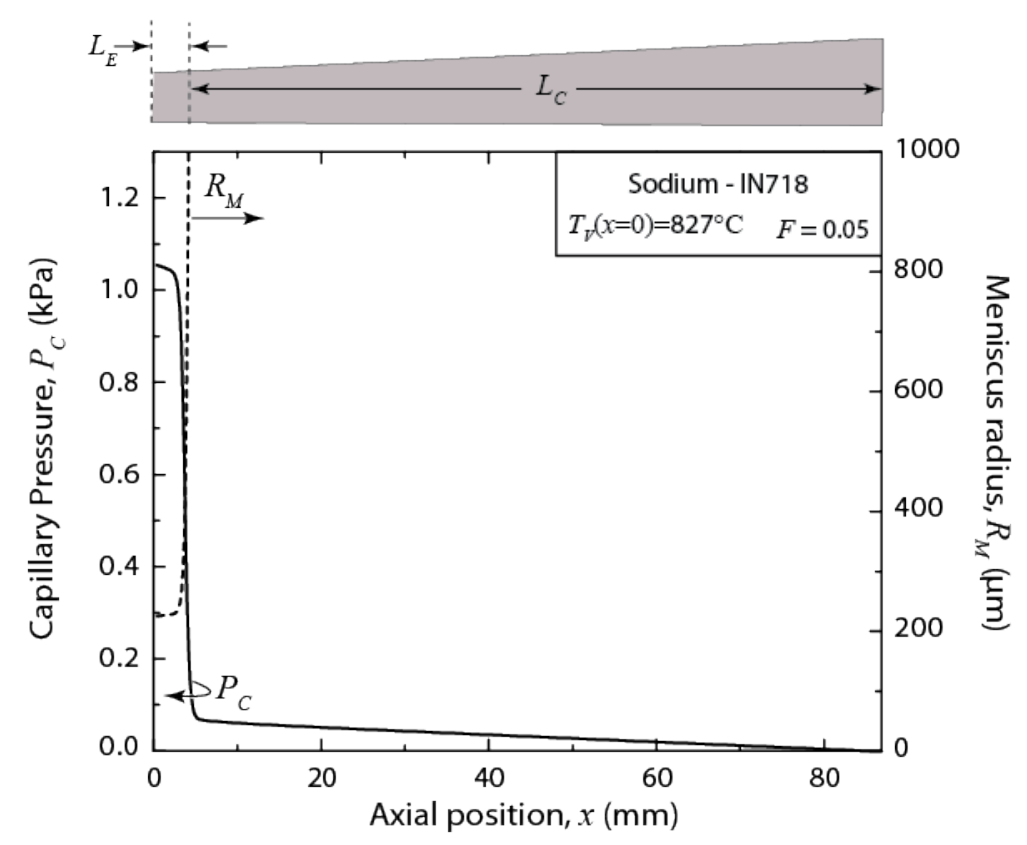

Figure 4.27. Capillary pressure (left ordinate) and meniscus radius (right ordinate) as a function of axial position for the Sodium-IN718 leading edge operating at its capillary limit of

$$
q_{\text {cap }}=217.1 \mathrm{~kW} / \mathrm{m}^{2} \text {. }
$$

Figure 4.28 shows the axial temperature distribution for $T_{W}, T_{W L}, T_{L V}$, and $T_{V}$. The plot for $T_{W}$ shows a steep temperature gradient along the wall at the tip but a relatively isothermal condenser section. $T_{V}$ remains nearly isothermal throughout the entire vapor stream. The positive differences $T_{W}-T_{W L}$ and $T_{W L}-T_{L V}$ over the curved tip drive heat through the wall and wick, respectively, into the system and the negative differences drive heat out of the system over the flat portion. 


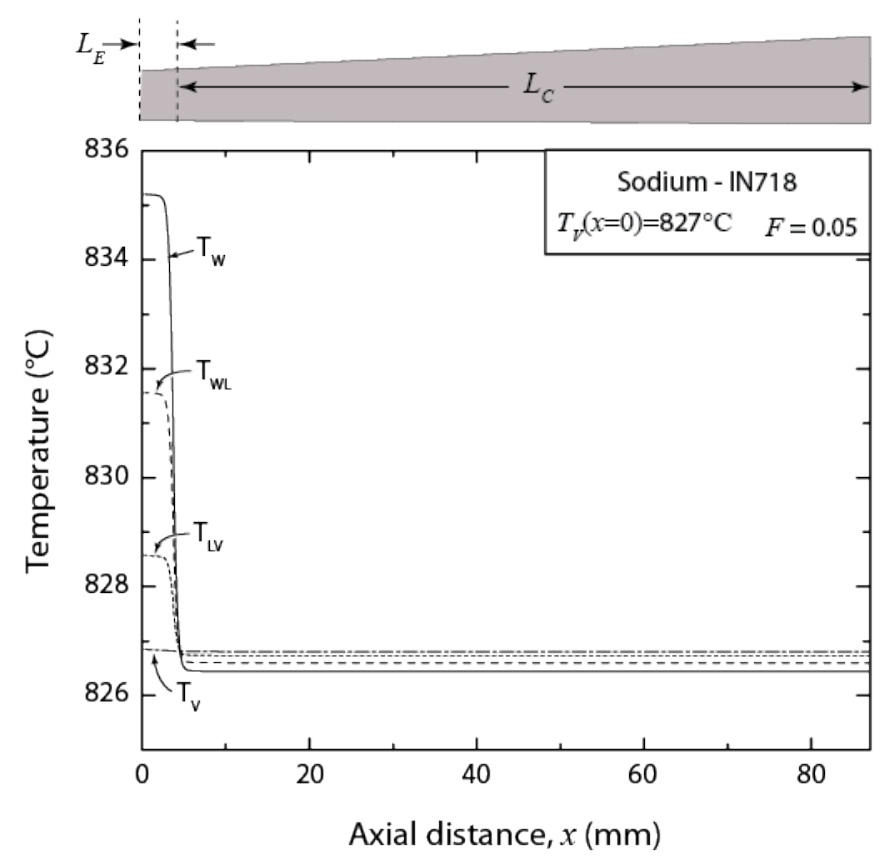

Figure 4.28. Axial temperature profiles for the Sodium-IN718 leading edge operating at its capillary limit of $q_{\text {cap }}=217.1 \mathrm{~kW} / \mathrm{m}^{2}$.

A parameterization of $L_{\text {flat }}$ and $b_{\text {wall }}$ was studied to understand their influence on the capillary limit. The parameter map of Figure 4.29 plots lines of constant $q_{w}$ over the design space of $20 \mathrm{~mm} \leq L_{\text {flat }} \leq 120 \mathrm{~mm}$ and $1.2 \mathrm{~mm} \leq b_{\text {wall }} \leq 1.8 \mathrm{~mm}$. Increasing the wall thickness always decreases the capillary limit. The temperature of the liquid-vapor interface, $T_{L V}$, is elevated at the tip to maintain the heat balance through the wall and wick. The corresponding saturation pressure, $P_{L V-E Q}$, is likewise elevated, lowering the capillary limit. It is therefore desirable to use the thinnest wall possible subject to the boiling and yielding limits (presented in the next chapter). 
Over most the design space $\left(b_{\text {wall }}<1.7 \mathrm{~mm}\right)$, there is an optimum $L_{\text {flat }}$ which maximizes the capillary limit. For example, at $b_{\text {wall }}=1.45 \mathrm{~mm}$, the optimum value is $L_{\text {flat }}=66 \mathrm{~mm}$. Lengthening $L_{\text {flat }}$ lengthens the liquid flow path which increases the liquid pressure drop and therefore reduces the capillary limit. Shortening $L_{\text {flat }}$ increases the condensation rate because heat must be expelled from the flat portion of the leading edge at a faster rate. Increases in condensation rate come from decreases in the first term of $\frac{P_{L V-E Q}}{T_{L V}{ }^{1 / 2}}-\frac{P_{V}}{T_{V}{ }^{1 / 2}}$ through the lowering of $P_{L V-E Q}$ in the condenser, increasing the capillary pressure necessary to stabilize the interface. This has the effect of lowering the capillary limit.

For thick walls $\left(b_{\text {wall }}>1.7 \mathrm{~mm}\right)$, lengthening the flat portion always elevates the heat transport capacity. Benefits of lowering the condensation rate (i.e. increasing $P_{L V-E Q}$ in the condenser to offset elevated values of $P_{L V-E Q}$ in the evaporator due to thick walls) always outweigh the disadvantage of increasing the liquid pressure drop. 


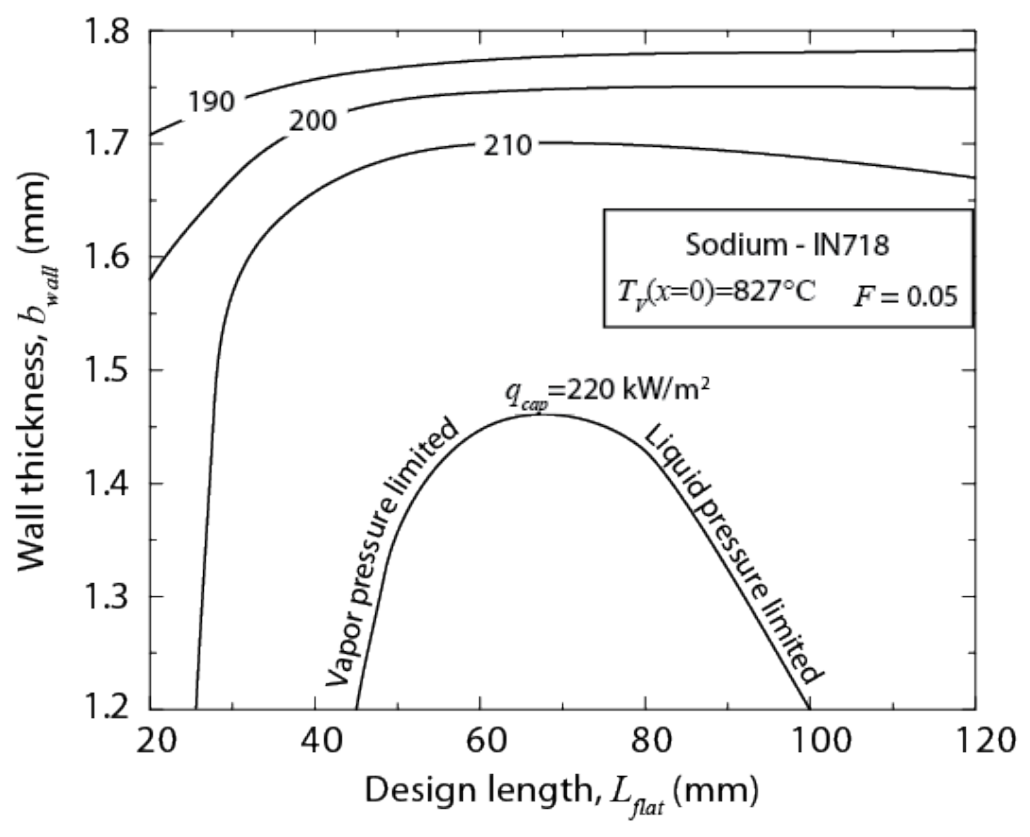

Figure 4.29. Parameter map for $L_{\text {flat }}$ and $b_{\text {wall }}$ showing iso-flux lines for $q_{c a p}$.

\section{$\underline{4.3(g) \text { Summary }}$}

With a proper formulation in place for the capillary limit, the other heat transport limits - sonic limit, yielding limit, and boiling limit - are presented in the next chapter. All four limits are considered collectively for the design of a high temperature leading edge heat spreader. 


\section{Chapter 5. Heat transfer limits}

Heat pipes fail when they encounter heat transport limits due to physical phenomena

linked to the sound speed in the tube (sonic limit), capillarity, and boiling. Well designed heat pipes avoid these failure mechanisms under expected operating conditions. Analytical models for the sonic, capillary, and boiling limits have been presented in numerous publications for cylindrical heat pipes [72], [73], [74], [107]. These models have not been adapted to the wedge geometry of interest where heat enters at a sharp tip and is transported through a variable cross-sectional area.

A design methodology is developed here which uses the intended flight parameters (Mach number and altitude), geometry (overall length, wall and wick thicknesses), and materials (working fluid, wick structure, solid case material) to identify bounded operating conditions for a wedge heat pipe. The three material systems - IN718-sodium, C103- 
lithium, and TZM-lithium - are examined in detail for their potential to manage hypersonic heat inputs for flight at Mach $5(24.5 \mathrm{~km})$ to $12(36.3 \mathrm{~km})$.

In addition to the sonic, capillary, and boiling limits, there is a fourth limit, yielding, which has not been discussed in the literature but that is relevant to cylindrical, or planar, or leading edge (wedge) heat spreaders. The basic theory for the yielding limit was presented in Chapter 3 and is extended here. The sonic limit, modified capillary limit, boiling limit, and yielding limit can be compiled into a design map that defines the maximum heat transfer capabilities of a wedge heat spreading system.

\subsection{Sonic limit}

In the vapor space at the tip $(x=0)$, the mass flow rate has been assumed zero (no slip condition) and increases in the axial direction (with $x$ ) as liquid is evaporated and injected into the vapor stream. At the end of the evaporator $\left(x=L_{e}\right)$, the vapor mass flow rate reaches a maximum. In cylindrical heat pipes with constant cross-sectional area, this location corresponds to the location of maximum vapor velocity. If the flow approaches the speed of sound in the vapor, a choked flow condition develops and this limits a further increase in the mass (and therefore thermal) transport rate. This is the well-known sonic limit[72], [73], [74], [107], [126] and is encountered in heat pipes subjected to high heat fluxes and operating at low vapor densities. 
Levy [126] first identified the sonic limit for cylindrical heat pipes. This limit establishes the maximum power, $Q_{\text {sonic }}$, which can be transported through the device. Levy [126] proposed an expression for the maximum power;

$$
Q_{\text {sonic }}=A_{V} \lambda \rho_{V}(\mathrm{x}=0)\left[\frac{\gamma R T_{V}(\mathrm{x}=0)}{2(\gamma+1) M}\right]^{1 / 2}
$$

where $\rho_{V}$ and $T_{V}$ are the density and temperature values at $x=0, R$ is the universal gas constant, $M$ is the fluid's molar mass, $\gamma$ is the vapor's heat capacity ratio, $\lambda$ is the fluid's latent heat of vaporization, and $A_{V}$ is the cross-sectional area of the vapor space.

To extend the limit to the wedge-shape design, we consider the case of a diverging duct with no mass injection or suction. When $M_{V}<1$, the duct acts as a subsonic diffuser with the velocity decreasing along $x$ [93]. When $M_{V}>1$, the duct acts as a supersonic nozzle, increasing the velocity along the $x$ direction [93]. If we now consider liquid molecules evaporating and entering the vapor stream, the mass injection leads to an increase in vapor velocity which, when subsonic, is slowed by the diverging vapor space. However, if enough mass is injected, the velocity can reach $M_{V}=1$. If still more mass is injected, the velocity can exceed the speed of sound, $M_{V}>1$, and the diverging space will accelerate the vapor. As a result, the wedge-shaped heat pipe could accelerate vapor supersonically without shock formation. In such a circumstance, choking is avoided in the evaporator and the heat spreader operates above its sonic limit. However, this is only possible if the heat flux is known beforehand, since the wedge would require a matching half angle which avoids shock formation based on the mass injection rate. Additionally, the vapor velocity will eventually 
decrease further down the wedge as vapor molecules condense and pressure is recovered, generating a shock in the condenser as the flow decelerates from supersonic to subsonic velocity .

Since aerodynamic requirements are likely to determine the wedge half angle, we instead take a simpler, conservative approach to model the sonic limit.. In this approach, we assume that, should the incident heat flux be high enough, the vapor's velocity will first reach $M_{V}$ at $x=L_{e}$, where the vapor's mass flow rate is largest. Replacing $A_{V}$ in the constant duct equation ((5.1)) with $A_{V}\left(x=L_{e}\right)$ (see equation (4.53)) and dividing by the external surface area which is subjected to the heat flux, $2 W R_{L E}\left(\frac{\pi}{2}-\varphi\right)^{12}$ gives an expression for the sonic heat flux limit;

$$
q_{\text {sonic }}=\frac{H_{V}(x=0) \lambda \rho_{V}(x=0)}{R_{L E}\left(\frac{\pi}{2}-\varphi\right)}\left[\frac{\gamma R T_{V}(\mathrm{x}=0)}{2(\gamma+1) M}\right]^{1 / 2}
$$

The only geometric variables which influence the sonic limit is the vapor space height, $H_{V}$ (which is dependent on half angle $\varphi$ ) and the leading edge radius, $R_{L E}$. The temperature and pressure dependence arises from the thermo-physical properties of the working fluid (assuming saturation), so the choice of fluid is important. Figure 5.1 shows the variation of $q_{\text {sonic }}$ with vapor temperature for sodium and lithium working fluids. Sodium outperforms

${ }^{12}$ By dividing by $2 W R_{L E}\left(\frac{\pi}{2}-\varphi\right)$, we have assumed that the stagnation point heat flux is constant over the curved region. Again, this will yield a conservative estimate of the sonic limit. 
lithium because it has a higher vapor density, particularly at lower temperatures (at $627^{\circ} \mathrm{C}$ $\left.\rho_{V, N a}=1.68 \times 10^{-2} \mathrm{~kg} / \mathrm{m}^{3}, \quad \rho_{V, L i}=1.17 \times 10^{-6} \mathrm{~kg} / \mathrm{m}^{3}\right)$, even though lithium has a much larger latent heat of vaporization $\left(\rho_{V, N a}=4.1 \times 10^{6} \mathrm{~J} / \mathrm{kg}, \rho_{V, L i}=2.1 \times 10^{8} \mathrm{~J} / \mathrm{kg}\right)$ and sound wave propagation rate, $\mathrm{c}=\left[\frac{\gamma R T_{V}}{M}\right]^{1 / 2}\left(c_{N a}=635 \mathrm{~m} / \mathrm{s}, c_{L i}=1182 \mathrm{~m} / \mathrm{s}\right)$.

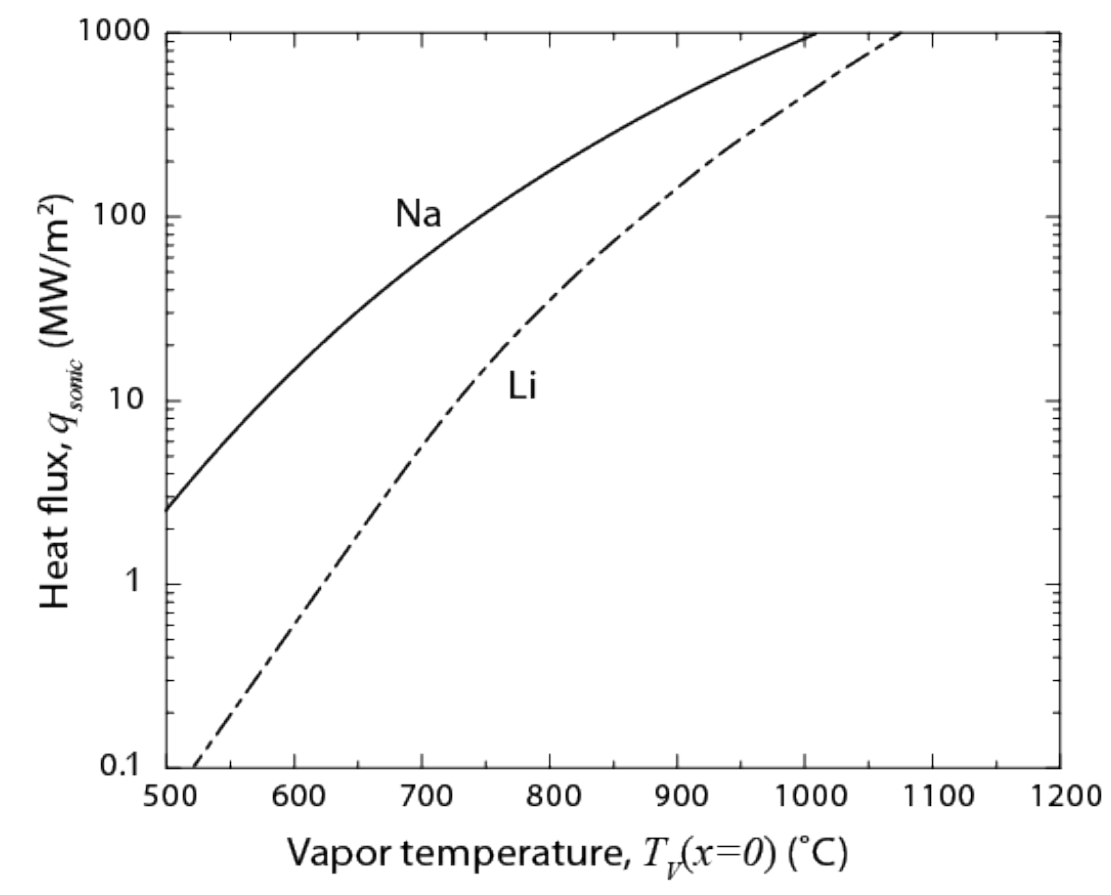

Figure 5.1. The maximum heat flux subject to the sonic limit using sodium and lithium as working fluids. 


\subsection{Yielding limit}

If yielding of the case material is to be avoided at a prescribed heat flux (i.e. flight Mach number and altitude), design choices for the wall alloy, wall thickness, $b_{\text {wall }}$, and flat surface length, $L_{\text {flat }}$, must keep the in-plane wall stress below its (temperature dependent) elastic limit. The stress model was presented in Chapter 3 to explore the influence of these parameters for a given flight condition (Mach number and altitude).. However, if wall material and values for $b_{\text {wall }}$ and $L_{\text {flat }}$ are known but flight conditions are not, a yielding limit can be recast (as follows) to identify the maximum wall heat flux (and therefore Mach number and altitude) that can be sustained.

We start with expression (3.29) which shows that the in-plane compressive stress induced at the tip is proportional to the through-thickness temperature difference;

$$
\sigma_{t h}=\frac{E \alpha\left(T_{\max }-T_{i s o}\right)}{1-\nu}
$$

Using the Tresca yield criterion, $\sigma_{y}=\sigma_{t h}$. Substituting and rearranging gives;

$$
T_{\text {max }}-T_{i s o}=\frac{\sigma_{y}(1-\nu)}{E \alpha}
$$

which is the maximum temperature difference which can be supported by the wall. Increases to $T_{\max }-T_{i s o}$ will cause compressive yielding of the wall. The heat flux through the curved tip of the wedge is governed by[15]; 


$$
q_{w}(x=0)=\frac{k_{\text {wall }}\left(T_{m a x}-T_{i s o}\right)}{R_{L E} \ln \left(\frac{R_{L E}}{R_{L E}-b_{w a l l}}\right)}
$$

Substituting equation (5.4) for the temperature difference gives;

$$
q_{\text {yield }}=\left(\frac{k_{\text {wall }} \sigma_{y}(1-\nu)}{E \alpha}\right)\left(\frac{1}{R_{L E} \ln \left[\frac{R_{L E}}{R_{L E}-b_{\text {wall }}}\right]}\right)
$$

Equation (5.6) is an expression for the maximum heat flux subject to face-sheet yielding. Any further increases in heat results in plastic deformation in the case wall at the leading edge tip. The first term on the right hand side of the expression consists of a combination of material properties while the second term contains the heat pipe design variables. The yielding limit is independent of working fluid and wick properties. The relevant temperature dependent material properties (elastic modulus, yield strength, thermal expansion, and thermal conductivity) of the three example alloys (IN718, C-103, and TZM) are summarized in Appendix A

Figure 5.2 shows how wall thickness and operating temperature affect the yield limit for IN718, C-103, and TZM case materials. The yield limit decreases with temperature primarily because of the increase in stress and decrease in $\sigma_{y}$, although decreasing stiffness helps abate the thermal stress somewhat. Over the analyzed design space in Figure 5.2, TZM outperforms IN718 and C103 due to its combination of high yield strength and high thermal conductivity. In the limit of $b_{\text {wall }} \rightarrow 0$, then $q_{\text {yield }} \rightarrow \infty$ for all cases; therefore, 
for very thin walls, the choice of case material becomes inconsequential for designing against the yielding limit. 

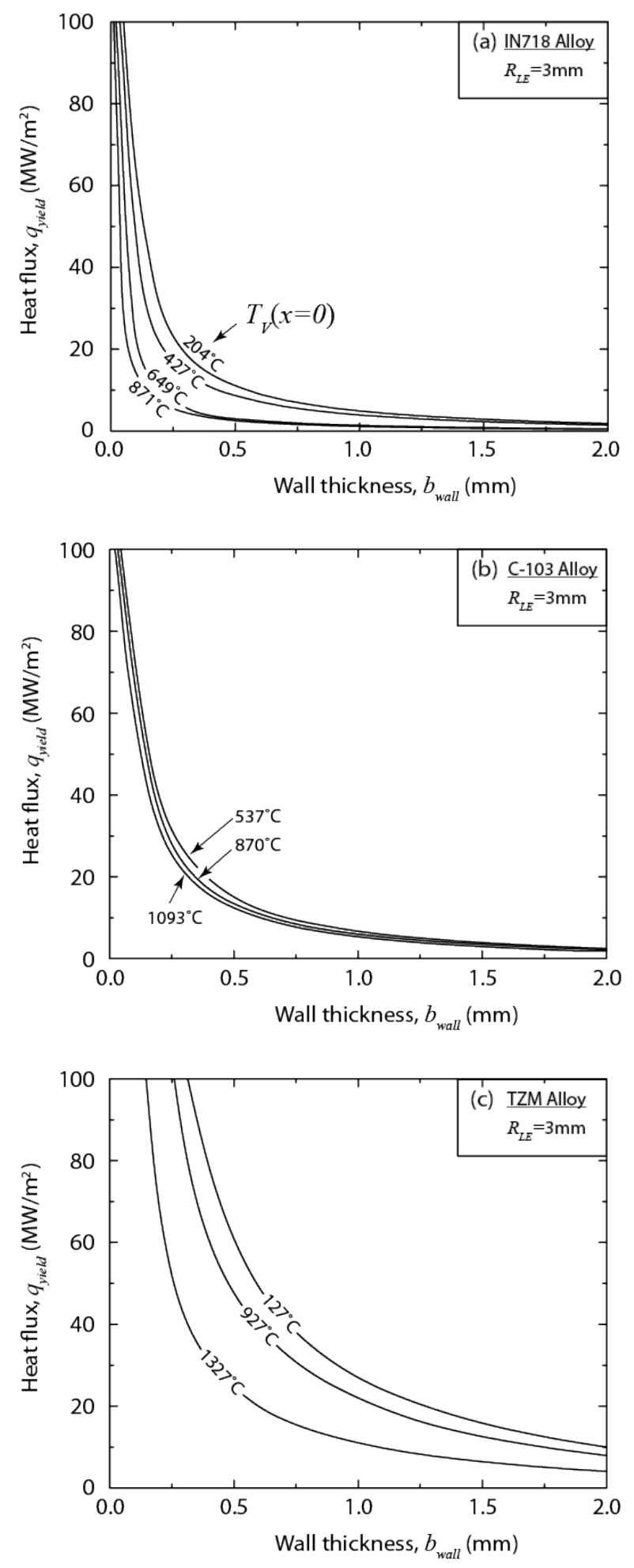

Figure 5.2. Influence of wall thickness and vapor stagnation temperature $(a t x=0)$ on the yielding limit for a (a) IN718, (b) C-103, and (c) TZM case material. 


\subsection{Boiling limit}

At higher operating temperatures, boiling of the fluid can restrict the heat transfer rate as bubbles nucleate in the wick, hindering the return of liquid to the evaporator [72], [73], [74], [94], [127]. Additionally, bubbles which form at the wall/wick interface create a region of high thermal resistance which inhibits heat flow to the liquid surface where evaporation occurs. Both situations result in local increases in temperature at the leading edge tip.

As schematically shown in Figure 5.3, heat is conducted through the case wall to the solid-liquid interface. If enough heat is transferred to the liquid, the liquid temperature will exceed its saturation temperature, $T_{L, s a t}$. The temperature rises to $T_{b}$ (the hottest liquid is immediately adjacent to the solid) and, at a high enough superheat, $\Delta T_{b}=T_{b}-T_{L, \text { sat }}$, a vapor bubble is formed [108]. 


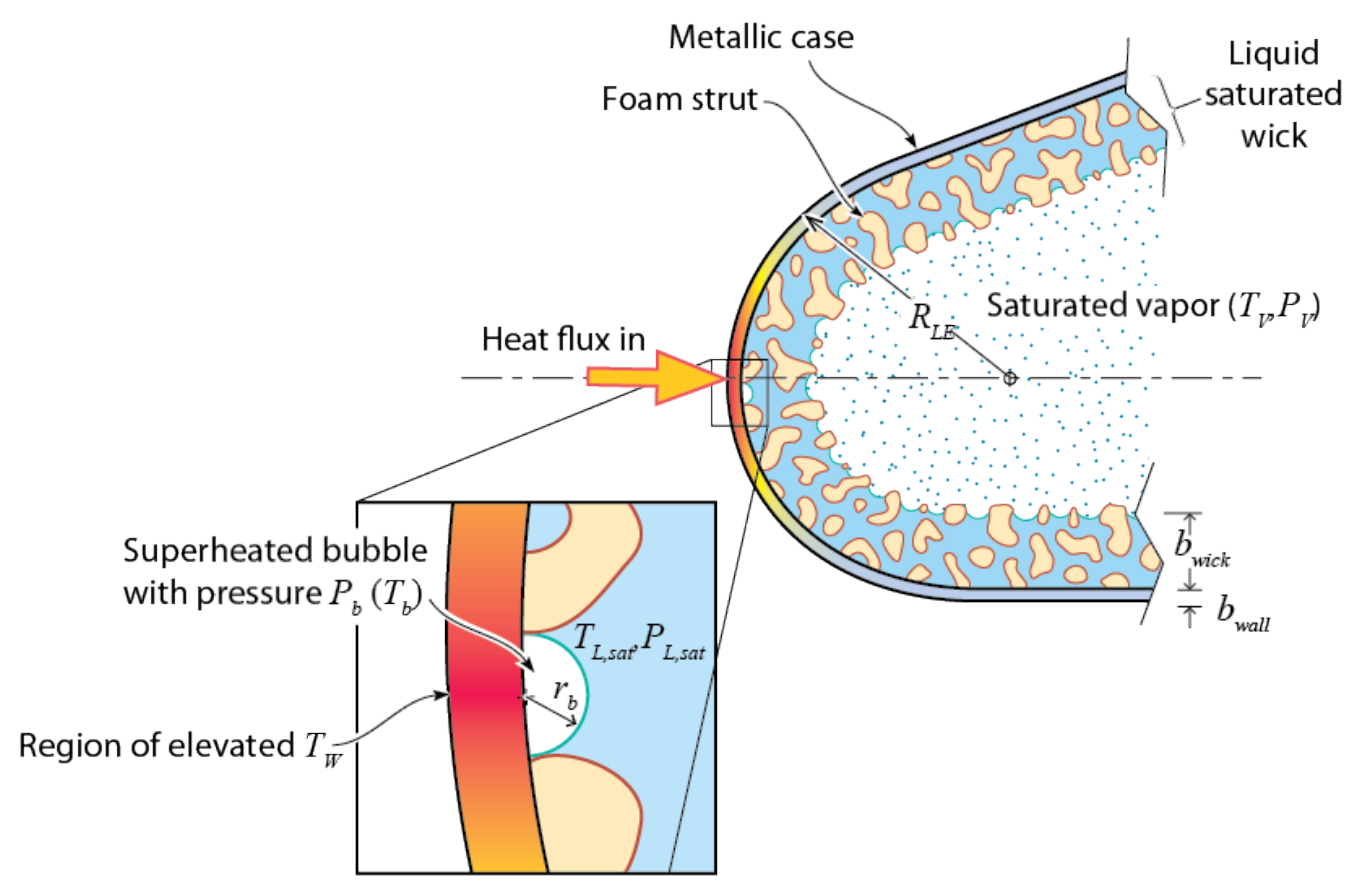

Figure 5.3. Nucleate boiling in the wick at the leading edge tip leading to a dryout condition.

A number of authors have developed expressions for the superheat required for boiling in a heat pipe wick[72], [73], [74]. Faghri [73] provides the critical superheat as a function of the liquid-vapor surface tension $\sigma_{L V}$, latent heat of vaporization $\lambda$, and bubble radius $r_{b}$;

$$
\Delta T_{b}=\frac{2 \sigma_{L V}}{r_{b} \rho_{V, o}} \frac{T_{V}(\mathrm{x}=0)}{\lambda}
$$

where $T_{V}(\mathrm{x}=0)$ and $\rho_{V, o}$ are the vapor stagnation temperature and density at the tip. Faghri [73] recommends $r_{b}=10^{-7} \mathrm{~m}$ for a conservative estimate. 
Using Fourier's law of conduction for a hollow cylindrical body with radial heat transfer [15], the wall heat flux transported through the wall that nucleates boiling conditions can be written as;

$$
q_{\text {boil }}=\frac{k_{\text {wick }} \Delta T_{b}}{\left(R_{L E}-b_{\text {wall }}\right) \ln \left(\frac{R_{L E}-b_{\text {wall }}}{R_{L E}-b_{\text {wall }}-b_{\text {wick }}}\right)}
$$

where $k_{\text {wick }}$ is the saturated wick thermal conductivity which. To first order, the wick thermal conductivity can be estimated using a rule of mixtures [72];

$$
k_{w i c k}=k_{S}(1-\varepsilon)+k_{L} \varepsilon
$$

where $k_{S}$ is the thermal conductivity of the solid wick material, $k_{L}$ is that of the liquid, and $\varepsilon$ is the wick porosity. Here, a high porosity, open pore metal foam is assumed with $\varepsilon=0.97$

The influence of wick thickness on the boiling limit is shown in Figure 5.4. As the wick is made thicker (longer conduction pathway), the boiling limit decreases considerably. Consequently, even small changes in wick thickness can have profound effects on the boiling limit. Despite differences in the thermal conductivity of C-103 and TZM, their boiling limits are nearly identical when the wick porosity is large (here, $\epsilon=0.97$ ). This is because the through-thickness thermal conductivity of the wick then becomes dominated by the thermal conductivity of the working fluid (equation (5.9)). 


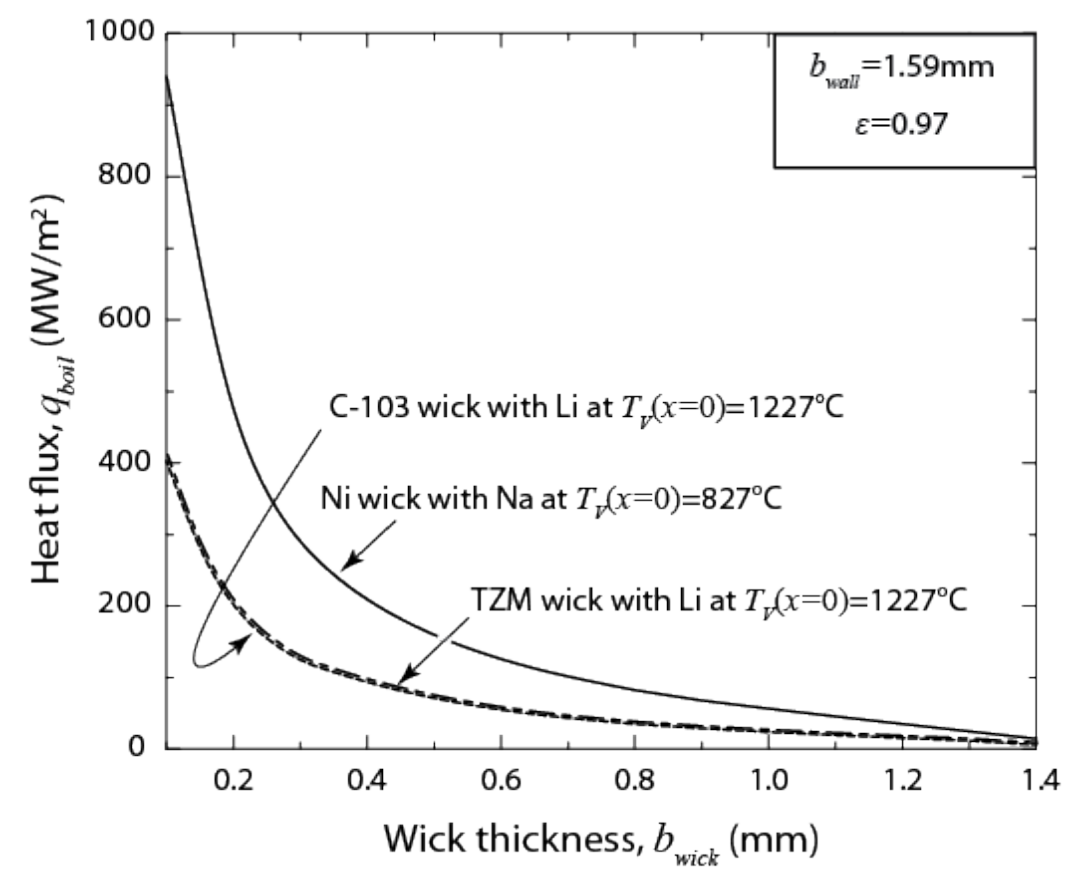

Figure 5.4. The maximum heat flux subject to wick boiling plotted as a function of wick thickness for a sodium-saturated nickel wick at $827^{\circ} \mathrm{C}$ and lithium-saturated $C-103$ and TZM wicks at $1,227^{\circ} \mathrm{C} . R_{L E}=3 \mathrm{~mm}$.

\subsection{Leading edge limit maps}

To explore the operating window, Figure 5.5 through Figure 5.7 show heat transport maps bounded by the capillary, yielding, sonic, and boiling limits for the Inconel 718sodium system, C103-lithium system, and TZM-lithium system, respectively. The relevant geometric parameters for a notional wedge heat dissipater are provided in Table 5.1. 
Leading edge radius, $R_{L E} \quad 3 \mathrm{~mm}$

Flat surface length, $L_{\text {flat }} \quad 82.3 \mathrm{~mm}$

Half angle, $\varphi \quad 6 \mathrm{deg}$

Working Fluid (See Appendix B for thermo-physical property data)

Working fluid type Sodium and Lithium, as noted

Case Design (See Appendix A for thermo-physical property data)

Case material

Wall thickness, $b_{\text {wall }}$

IN718, C-103, \& TZM, as noted

Case width, $W$

$1.59 \mathrm{~mm}$

$21.2 \mathrm{~mm}$

\section{Wick Design}

Wick material

Wick thickness, $b_{\text {wick }}$

Wick permeability

Pore radius, $R_{P}$

Porosity, $\epsilon$
Nickel, Niobium, and Molybdenum, resp.

$0.71 \mathrm{~mm}$

$7.74 \times 10-9 \mathrm{~m} 2$

$225 \mu \mathrm{m}$

0.97

The sonic and boiling limits for the C103-lithium and TZM-lithium system are identical. This is because the sonic limit is independent of the structural case material, and while the boiling limit depends on the wick thermal conductivity, this is nearly identical for a high porosity wick using the same working fluid.

The IN718-sodium map plots the capillary limit for several effective evaporation fractions, $F$. Chi's capillary limit model would follow $F=1.0$ most closely, defining the upper bound on heat transport over the entire temperature range of 600 to $1000^{\circ} \mathrm{C}$. Using 
this model, along with the other classic models presented for the sonic and boiling limits, one would conclude that the leading edge could accommodate flight enthalpies up to Mach $7(29.0 \mathrm{~km})$ without failure. The operating envelope accommodates incent thermal fluxes as high as $3 \mathrm{MW} / \mathrm{m}^{2}$ at operating temperatures of $1000^{\circ} \mathrm{C}$.

Consider now the yielding limit proposed in this work, in combination with the Chi capillary limit. Below $780^{\circ} \mathrm{C}$, Chi's capillary limit restricts the flight envelope while above this temperature, performance is governed by the yield strength of the case material. Mach 7 flight $(29.0 \mathrm{~km})$ would cause plastic deformation at the tip. The maximum wall heat flux that can be sustained is $2.5 \mathrm{MW} / \mathrm{m}^{2}$ at $780^{\circ} \mathrm{C}$. At $1000^{\circ} \mathrm{C}$, it is $850 \mathrm{~kW} / \mathrm{m}^{2}$, a $72 \%$, drop when considering the yielding limit..

If the Chi limit is replaced by the modified version developed here, and an effective evaporation fractional area of $F=0.05$ is now considered, the flight operating regime is bounded by the capillary limit over the entire temperature range. The maximum wall heat flux of $200 \mathrm{~kW} / \mathrm{m}^{2}$ occurs at about $700^{\circ} \mathrm{C}$, significantly smaller than Chi model prediction.

Turning now to the C103-lithium system, flight envelopes up to Mach 8 (30.8km) can be accommodated without failure due to the higher high-temperature strength of C103. Over the entire temperature range, the upper limit of the flight operating regime is governed by yielding limit, suggesting a higher strength material paired with lithium could open up the operating envelope even more. 
One such higher strength material is TZM. Figure 5.7 shows its potential for accommodating heat fluxes over Mach $10(33.8 \mathrm{~km})$. The strength and thermal conductivity of TZM is high enough to push the yielding limit above the capillary limit. Lithium's favorable heat transfer properties maintain a high heat transport capacity.

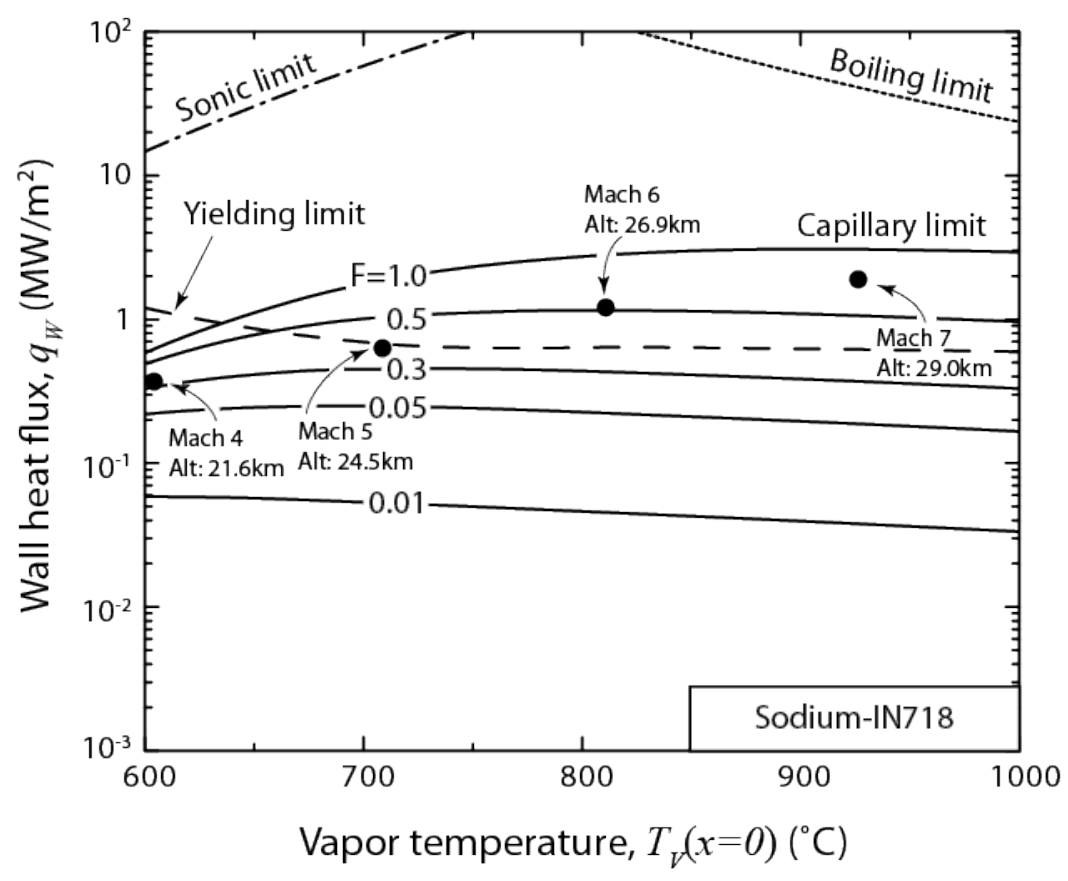

Figure 5.5. Operational map for the sodium-IN718 system. 


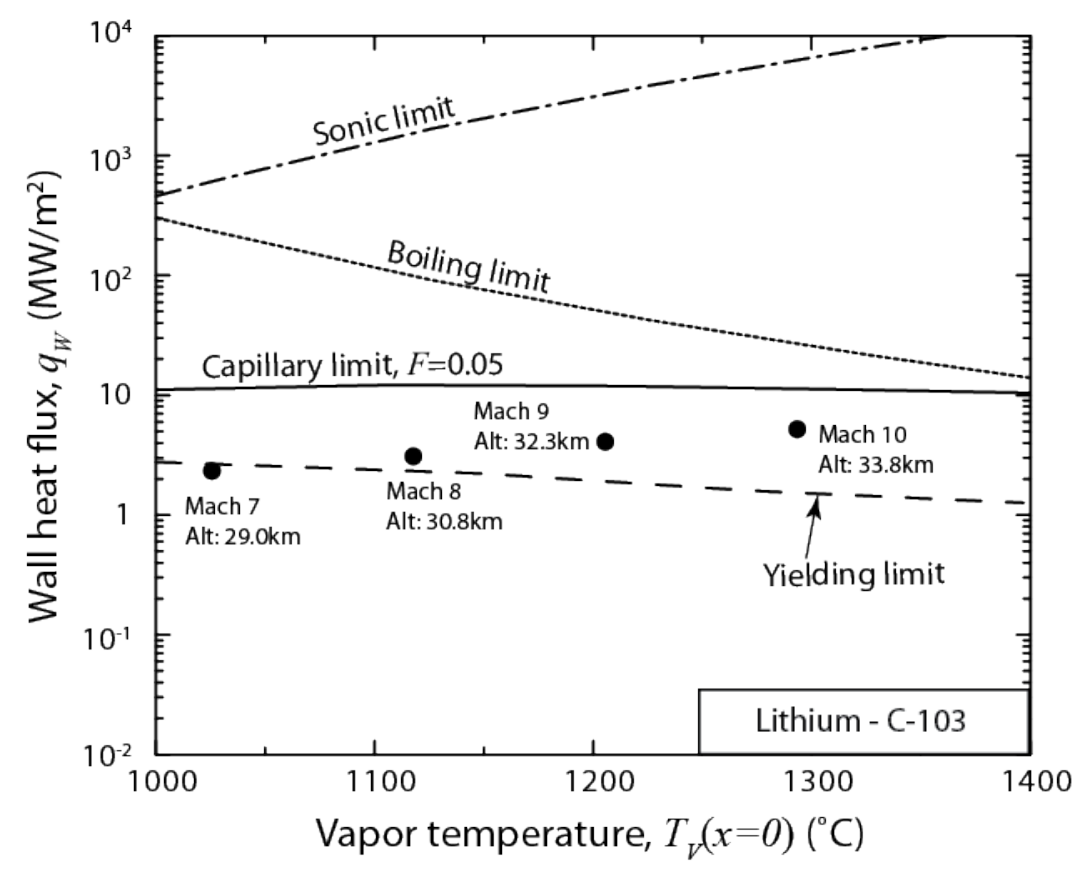

Figure 5.6. Operational map for the lithium-C103 system. 


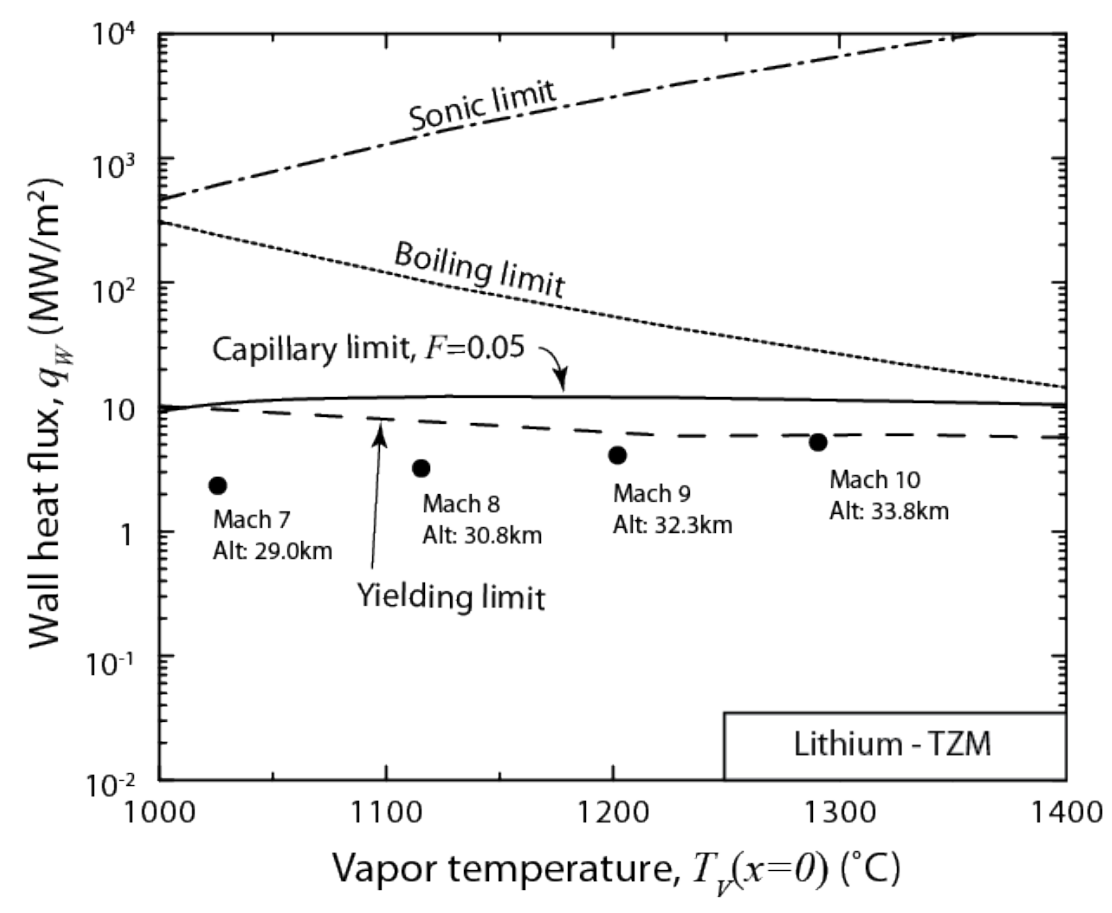

Figure 5.7. Operational map for the lithium-TZM system.

In summary, the proposed capillary (at $F=0.05$ ) and yielding limits restrict the operating region of all three systems: sodium-IN718, lithium-C-103, and lithium-TZM. The sonic and boiling limits do not restrict heat transport using the design parameters chosen here. Furthermore, the capillary and yielding limits are so restricting that the sonic and boiling limits are unlikely to be encountered in any aerodynamic leading edge design operating at steady state.

A sodium-IN718 system can be designed for low Mach number flight (less than Mach 5). For higher Mach numbers, the operating envelope is expanded to Mach 8 for a lithium-C103 material combination and up to Mach 10 for a lithium-TZM system. 


\section{Chapter 6. Low temperature evaluation}

To investigate the thermal spreading effectiveness of the leading edge concept and its potential for accommodating the intense, localized heating of the hypersonic environment, a low temperature, proof-of-concept leading edge heat spreader was experimentally explored. The design and fabrication of a stainless steel system utilizing water as the working fluid is described and its thermal performance is compared to an otherwise identical, but evacuated (no working fluid) test article. The results are used to test (1) a predictive Finite Element (F.E.) model that is presented which treats the vapor as a solid having very high thermal conductivity, and (2) the analytical design model presented in Chapter 3. 


\subsection{Leading edge design and fabrication}

The benefit of cellular materials which offer low resistance pathways for easy vapor flow while providing structural load support in two-phase heat spreaders has been described [84], [128]. In view of the discussion on the capillary limit (Chapter 4), a core design with low vapor drag allows the latent heat to be transported through the core with little resistance. The capillary limit can be increased by minimizing the product of the friction factor, $f$, and Reynolds number, $\operatorname{Re}_{\mathrm{V}}$ (see equation (4.54)). Figure 6.1 shows a map relating $f$ and $\operatorname{Re}_{\mathrm{V}}$, with the diagonal lines indicating constant contours of $f$ Re, for a variety of core concepts [129]. At low Reynolds numbers, the $f \cdot \operatorname{Re}_{\mathrm{V}}$ product is bound by $f \mathrm{Re}_{\mathrm{V}}=64$ for laminar flow within empty, circular cross-section channels (i.e. pipe flow) [93]. Of the cellular materials, corrugated ducts and louvered fin geometries have the lowest $f \cdot \operatorname{Re}_{\mathrm{V}}$ products ( $f \cdot \mathrm{Re}_{\mathrm{V}} \approx 90$ to 1500$)$ and would be preferred. While lattice frame materials (LFM's) and Kagome cores have better load supporting capacities, their products are $f \cdot \mathrm{Re}_{\mathrm{V}} \approx$ 1500 to $3 \times 10^{5}$ and introduce more drag, increasing the axial pressure drop which will reduce the capillary limit and narrow the operational window. 


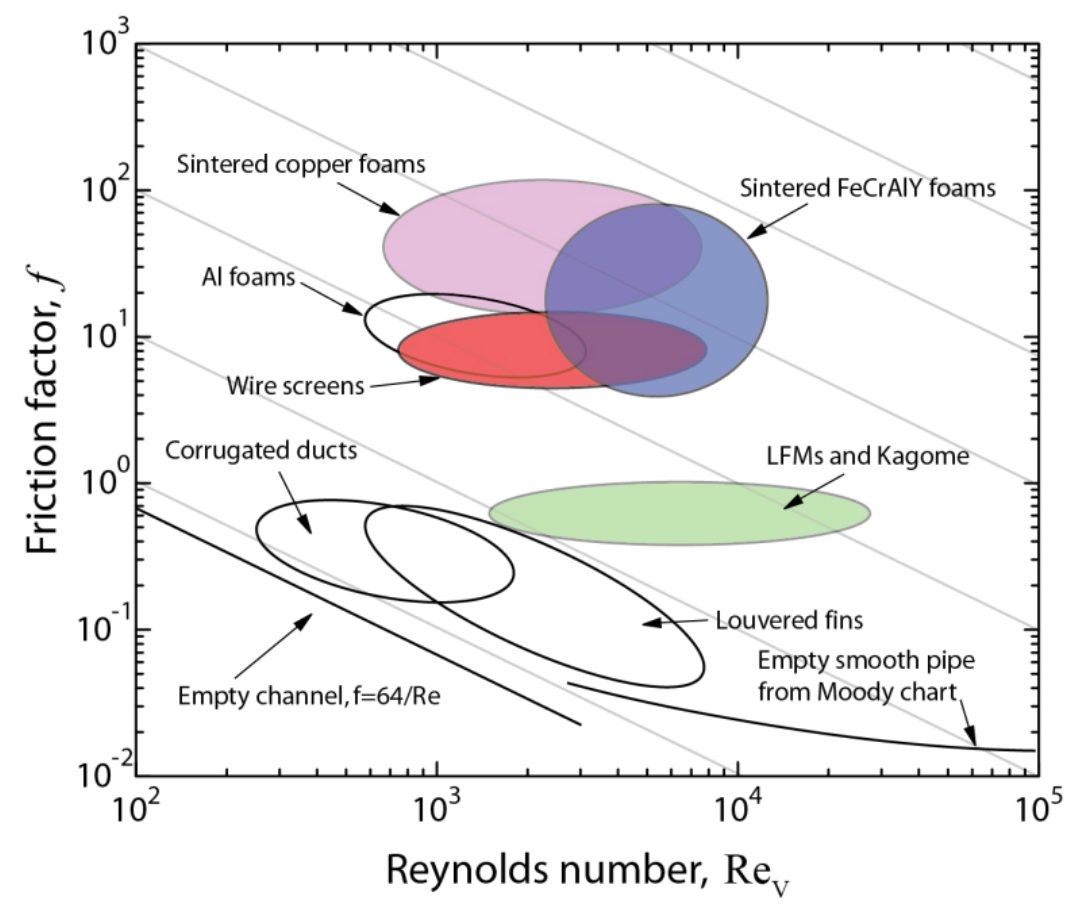

Figure 6.1. Friction factors for various core topologies as a function of the Reynolds number. The lightened diagonal lines indicate contours of constant $\left(f R_{V}\right)$. Adapted from [129].

Based upon the considerations above, a structural I-core with flow properties similar to a louvered fin channel was selected for investigation. The concept is shown in Figure 6.2. It consists of a diverging vapor space with vertical ribs, resulting in channels with low vapor flow resistance in the axial direction. Working fluid transport in this wedge design is achieved using a seamless, multi-layered screen wick lining the inner surfaces of the top and bottom face-sheets and the curved tip. The wick on the faces passes through voids notched at the base of the ribs to allow for transverse liquid flow paths between vapor cells. This arrangement also secures the wicking material against the face sheet, minimizing the thermal conduction resistance. Wicking material lined neither the vertical side walls nor vertical ribs. 


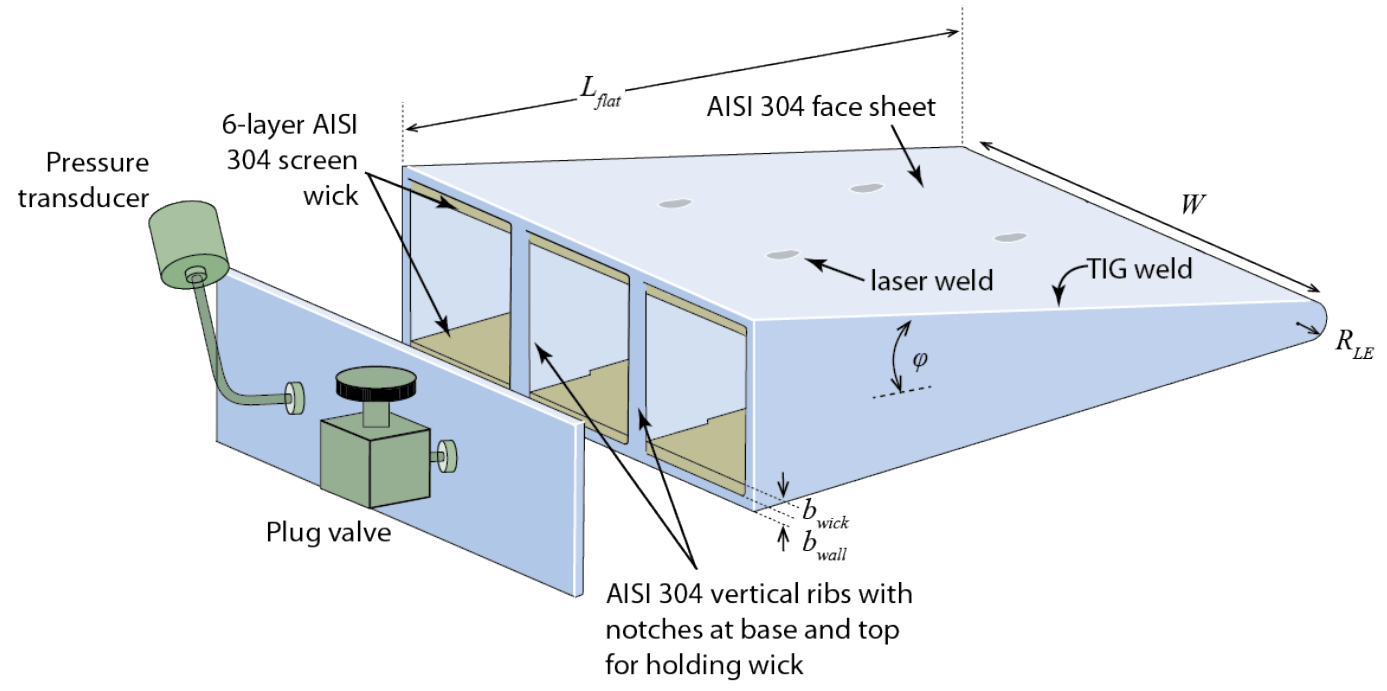

Figure 6.2. Schematic of the I-core leading edge design.

Face sheets for the leading edge were fabricated from $1.59 \mathrm{~mm}$ thick AISI 304 stainless steel sheet. This alloy was chosen for its good ductility, weldability, and compatibility with water working fluid plus corrosion inhibitor [74]. A sheet metal shear was used to cut the face sheets to final dimensions and a forming die was used to bend the tip to a leading edge outer radius of $7.86 \mathrm{~mm}$ and $6^{\circ}$ half angle. The side face plates, a rear face plate, and the vertical ribs were fabricated from $3.18 \mathrm{~mm}$ thick sheet of the same material and milled to final dimensions. A six-layer stacking arrangement using stainless steel woven screen (AISI 304, Cleveland Wire Cloth ${ }^{\circledR}$ ) having a mesh number of 100 was chosen for the wick. Manufacturer specifications list a wire diameter of $114 \mu \mathrm{m}$ and square openings with a side length of $140 \mu \mathrm{m}$. Measurements by microscopy confirmed these dimensions. Prior to final assembly, all parts and wicking material were immersed in a room temperature passivation bath (to remove iron particles adhered to surfaces) of 30vol\% nitric acid and 
70vol\% distilled water for 60 minutes [73]. After removal from the bath, the parts were rinsed with methanol and blown dry with dry nitrogen gas.

The side face plates were fusion welded to the edges of the formed face sheet using an argon-shielded TIG welder. The vertical ribs and wicking material was then slid into the assembly from the rear. A $2 \mathrm{~kW}$ (maximum) continuous solid-state Nd:YAG laser $(1064 \mathrm{~nm}$ wavelength) was used to laser weld the vertical ribs to the face sheet. Retaining clips were laser welded through the side face-sheets to restrain the wicking material against the inner surface of the upper and lower face sheets. The rear face plate was then TIG welded using the same process used for the side face sheets. After welding, the wedge leading edge was pressure proof tested to $275 \mathrm{kPa}$ using compressed air. A photograph of the system (prior to installation of the rear plate) is shown in Figure 6.3. Design values for the system is given in Table 6.1. 


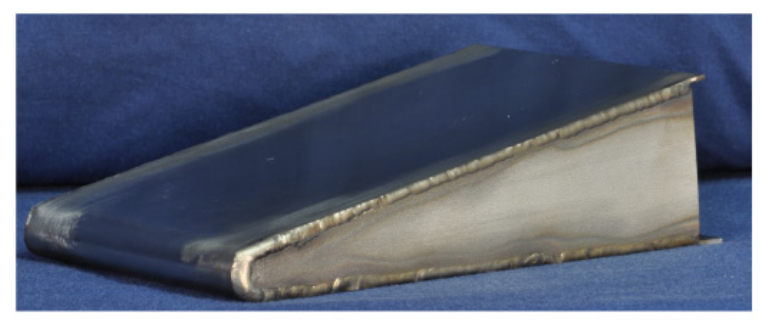

(a)

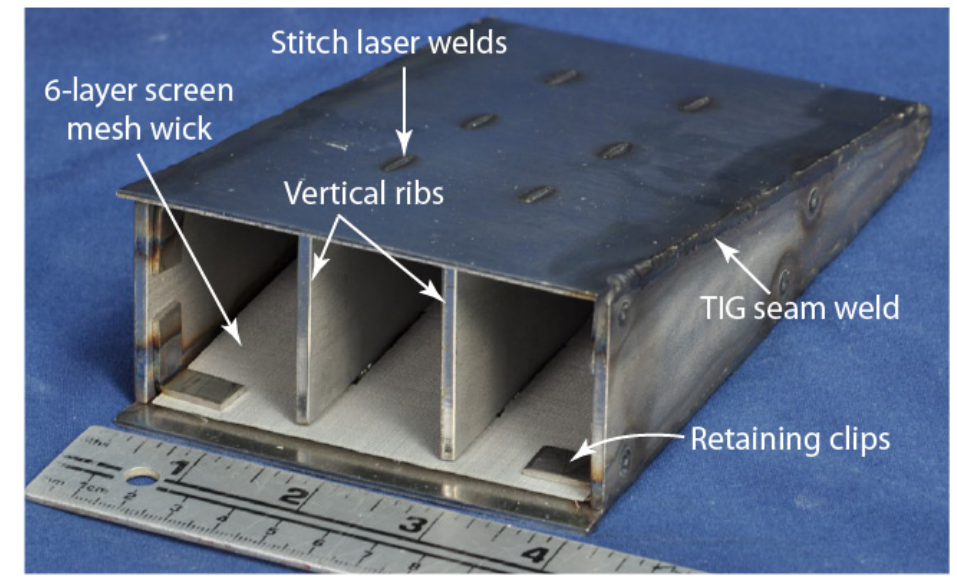

(b)

Figure 6.3. Photograph of the stainless steel leading edge system showing (a) the curved leading edge and (b) the internal core and wick (prior to assembly of the rear face sheet). 
Table 6.1. Relevant parameters for the low temperature leading edge heat pipe.

Working Fluid (See Appendix B for thermo-physical property data)

Working fluid type

Water + inhibitor

\section{Case Design}

Case material

AISI 304

Length of flat surface, $L_{\text {flat }}$

$160.3 \mathrm{~mm}$

Half angle, $\varphi$

$6^{\circ}$

Leading edge radius, $R_{L E}$

$7.86 \mathrm{~mm}$

Wall thickness, $b_{\text {wall }}$

$1.59 \mathrm{~mm}$

Width, $W$

$101.6 \mathrm{~mm}$

\section{Wick Design}

Wick material

AISI 304

Wick thickness (six layers), $b_{\text {wick }}$

$1.17 \mathrm{~mm}$

Wick porosity, $\varepsilon$

0.617

Pore radius (half wire opening), $R_{P}$

$70 \mu \mathrm{m}$

Wire diameter, $d_{w}$

$114 \mu \mathrm{m}$

An isolation ball valve (Nupro 304 SS, Kurt J. Lesker Company, Pittsburg, PA) was

temporarily installed to the rear plate for an initial hermicity test using a tracer-probe method with acetone (commonly referred to as an "acetone leak test"). Two leaks were discovered and re-welded. After the repair, a vacuum of less than $10^{-4}$ Torr was maintained for over 24 hours. The valve was then removed and all interior and exterior surfaces were rinsed with ethanol and baked out at $250^{\circ} \mathrm{C}$ at $10^{-4}$ Torr for 6 hours.

To monitor the internal vapor pressure during testing, a diaphragm-type pressure transducer (PX176 Series, Omega Engineering Inc., Stamford, CT), with a useable range of $5 \mathrm{kPa}$ to $34.5 \mathrm{kPa}$ and an error of $\pm 3.45 \mathrm{kPa}$, was connected to a tee fitting. The isolation 
valve was reinstalled but now into the tee fitting. A final leak check was performed by evacuating to less than $10^{-4}$ Torr and temporarily closing the valve. Changes to the vacuum level over a 24 hour period were monitored. A rise in internal pressure of less than $6.9 \mathrm{kPa}$ (twice the accuracy of the pressure gauge) over the 24 hour period was considered satisfactory.

Two test articles were fabricated. The first was evacuated to less than $10^{-4}$ Torr and sealed but not charged with a fluid. The second was also evacuated but then charged with a $20.1 \mathrm{~mL}$ mixture of $0.05 \% \mathrm{w} / \mathrm{v}$ potassium chromate $\left(\mathrm{K}_{2} \mathrm{CrO}_{4}\right)$ corrosion inhibitor and distilled water [74]. The chromate anion forms an adherent passivating layer of chromium III oxide $\left(\mathrm{Cr}_{2} \mathrm{O}_{3}\right)$ or chromium II hydroxide-chromate $\left(\mathrm{Cr}(\mathrm{OH}) \mathrm{CrO}_{4}\right)$ slowing the chemical reaction of iron with water which produces hydrogen by $\mathrm{Fe}+2 \mathrm{H}_{2} \mathrm{O} \rightarrow \mathrm{Fe}(\mathrm{OH})_{2}+\mathrm{H}_{2}$ [74]. Hydrogen acts as a gas buffer in the vapor core which reduces the extent of isothermalization. The fill volume amount equaled the calculated volumetric void space in the wick based on porosity measurements. The wick porosity is a function of its density $\left(\rho_{\text {wick }}\right)$ and that of the material from which it is made $\left(\rho_{\text {solid }}\right)$;

$$
\varepsilon=1-\frac{\rho_{\text {wick }}}{\rho_{\text {solid }}}
$$

The density of the solid material is a known material property while the density of the wick is determined from;

$$
\rho_{\text {wick }}=\frac{m}{A_{\text {wick }} b_{\text {wick }}}
$$


where $A_{\text {wick }}$ is the cross-sectional area and $b_{\text {wick }}$ the thickness of the wicking material, and $m$ is its mass. Note that for a multi-layer wick, $b_{\text {wick }}$ is its overall thickness and takes into account the random stacking alignment of the layers which introduce an added component to the porosity. A six-layer $70 \mathrm{~mm}$ by $90 \mathrm{~mm}$ sample coupon was weighed and its thickness measured at several locations. The average porosity was determined to be $\varepsilon=0.617$.

The backfill apparatus shown in Figure 6.4 was used for charging. The working fluid was held in a reservoir which is initially isolated from the vacuum lines. A vacuum turbo-pump evacuated the test article to less than $10^{-4}$ Torr which is then isolated from the vacuum system (by closing the vacuum isolation valve). The reservoir's metering valve was then opened, allowing fluid to flood the test article. The leading edge isolation valve was then closed and the sample detached from the backfill apparatus.

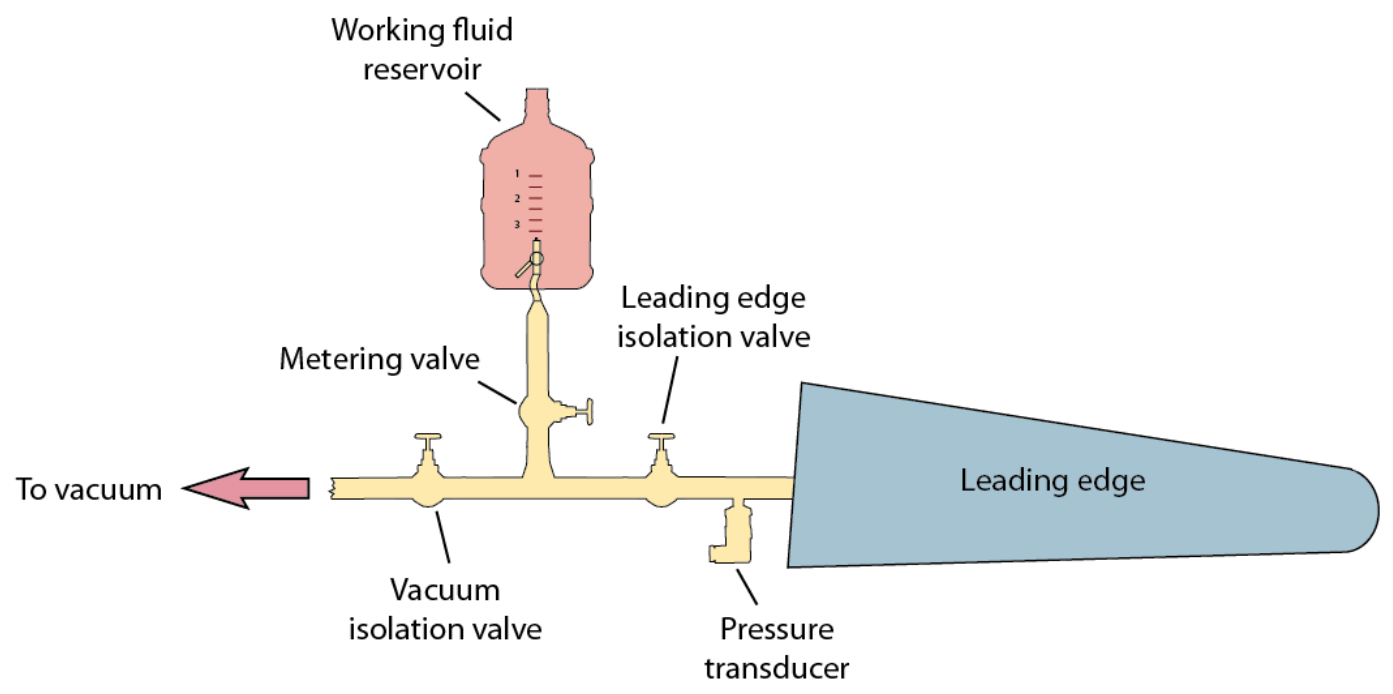

Figure 6.4. Evacuation and charging apparatus connected to the test article. 


\subsection{Experimental setup}

The test geometry is schematically illustrated in Figure 6.5 . Three $25.4 \mathrm{~mm}$ by 25.4mm self-adhesive thin-film polyimide heaters (KHLV-101, Omega Engineering ${ }^{\circledR}$ ) were affixed to the curved portion of the leading edge. A multimeter was used to measure the heating circuit resistance, $R_{c}$, and found to be $R_{c}=27.4 \Omega$. The voltage potential applied to the heating circuit could be adjusted using a variable A.C. transformer ( 0 to $26.5 \mathrm{~V})$ and its root mean square value, $V_{r m s}$, measured using the multimeter. The mean applied power to the heaters, $\bar{P}$, could then be calculated using;

$$
\bar{P}=\frac{V_{r m s}^{2}}{R_{c}}
$$

For insulation, a $9.6 \mathrm{~mm}$ thick rigid polyamide pressboard material with tradename Nomex $^{\text {TM }}$ Type 994 (DuPont ${ }^{\oplus}$ Advanced Fiber Systems, Richmond, VA) was adhered to the sides and rear of the test article using a room temperature vulcanizing silicone adhesive (Permatex ${ }^{\oplus}$, Hartford, CT ). The thermal conductivity of the insulation was given by the manufacturer as $0.16 \mathrm{~W} / \mathrm{mK}[130]$. Test samples were mounted from the rear fill tube on a laser leveling table and kept within $\pm 0.5^{\circ}$ of the horizontal (checked with a digital level).

Surface temperature measurements were taken by an infrared (IR) thermal camera (Model A325, FLIR ${ }^{\oplus}$ Systems Inc., Boston, MA, 7.5 to $13 \mu \mathrm{m}$ spectral range) mounted above the test sample with its field of view orthogonal to the flat surface of the leading edge. The camera was factory calibrated for operation within the 0 to $350^{\circ} \mathrm{C}$ range with error being the 
greater of $\pm 2^{\circ} \mathrm{C}$ or $2 \%$ of the reading. Imaged surfaces were painted with a high temperature, high emissivity paint $\left(\mathrm{Krylon}^{\circledR}\right.$, Cleveland, $\left.\mathrm{OH}\right)$ to set the surface emissivity to 0.95 for camera auto-correction. At the tip, infrared radiation from the heaters interfered with thermal camera readings so a type-K thermocouple (Omega Engineering ${ }^{\circledR}$, Stamford, CT) was adhered between two of the thin film heaters to obtain accurate tip temperatures. A second thermocouple was adhered to the surface of the thin film heater and a third thermocouple to the flat surface toward the rear for camera calibration. Differences between the IR readings and calibration thermocouple were found to be within $\pm 3^{\circ} \mathrm{C}$. Measurements of the vapor pressure were logged using the pressure transducer. An error analysis is presented in Appendix E. 


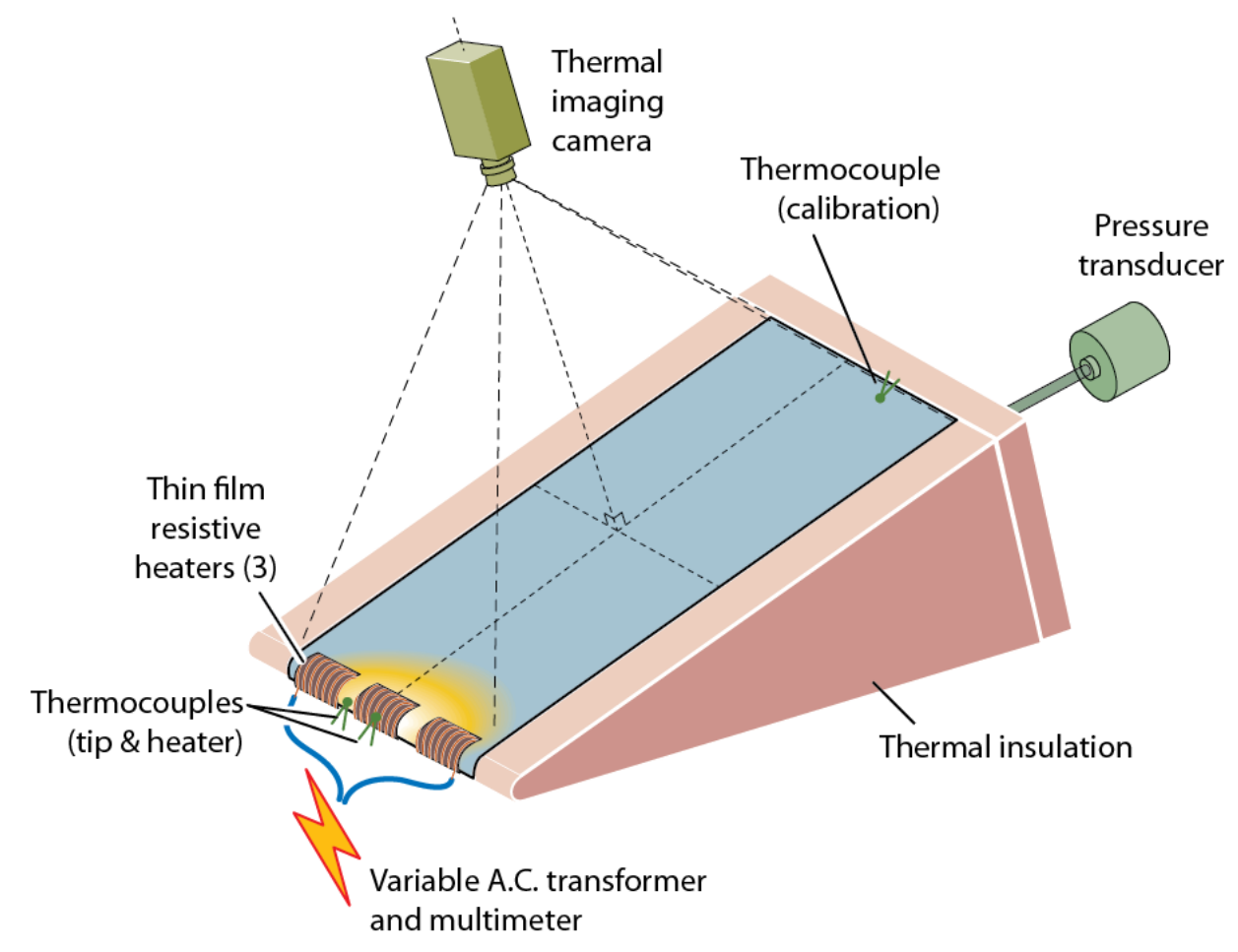

Figure 6.5. Experimental setup for testing the low temperature leading edge heat spreader.

\subsection{Experimental results}

Thermographs taken at steady state of the evacuated and charged leading edge top surfaces are shown in Figure 6.6 for applied powers of 5W, 15W, and 25W. A region of elevated temperatures arches over the heaters at the tip of the evacuated test article while no such bow is evident on the surface of the charged sample. The maximum surface temperatures are significantly reduced ( $43 \%$ reduction at $25 \mathrm{~W}$ using the tip's thermocouple reading) with no obvious evidence of dry-out (identified by a temperature spike at the tip). The charged sample's cooler tip temperature and warmer rearward temperatures demonstrate 
that the heat is being spread more efficiently in the heat spreader than in the evacuated system. Its isothermal operation suggests a high effective thermal conductivity. 
I. Evacuated (no working fluid) $\quad$ II. Charged (working fluid)

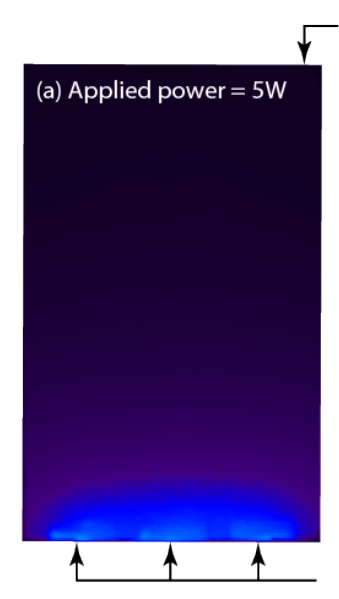

Rear edge $\neg$
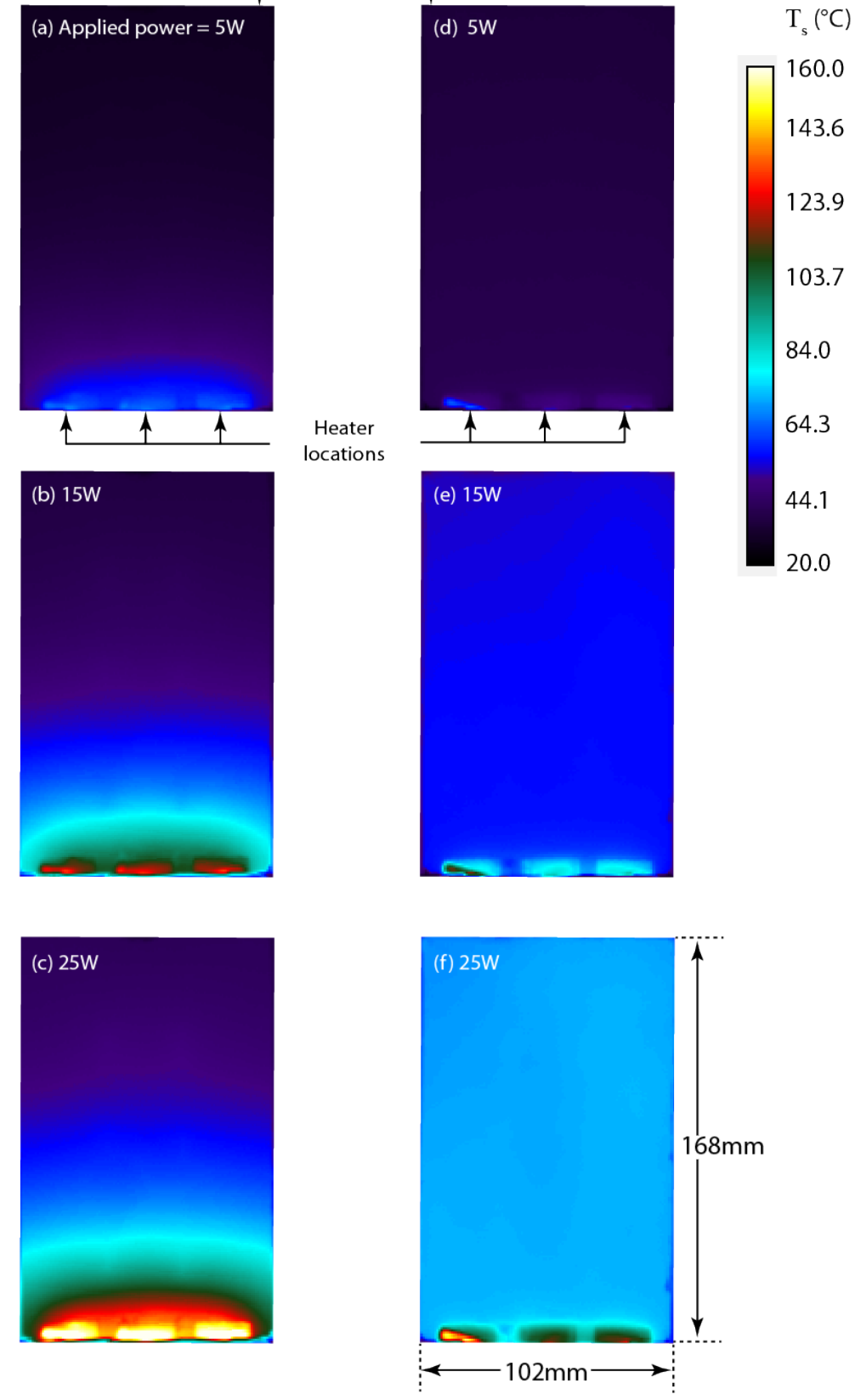

Figure 6.6. Steady state, top surface thermographs of (I) the evacuated and (II) charged leading edge at several applied power levels. 


\subsection{Thermal simulation model}

Near isothermal operating regime of heat spreaders, it is possible to model the vapor core as a solid thermal conductor of high conductivity. This approach predicts operational temperatures with reduced computational effort, avoiding the challenge of modeling twophase conjugate heat transfer. Unlike frequently used lumped capacitance models for modeling heat pipes [73], [84] which predict a completely isothermal system, the present model developed here seeks to capture thermal gradients near the tip which can be substantial during hypersonic heating (which exposes the tip to much higher heat fluxes than those explored here experimentally) despite the nearly isothermal response of the vapor core. Once steady state conditions are achieved, the 3D heat equation (with isotropic thermal conductivity) can be solved by finite element methods over each material component, including the vapor. For each material in the system, the governing equation is;

$$
0=k\left(\frac{\partial^{2} T}{\partial x^{2}}+\frac{\partial^{2} T}{\partial y^{2}}+\frac{\partial^{2} T}{\partial z^{2}}\right)
$$

where $T$ is the temperature of the wick, solid, or vapor, and $x, y, z$ are position coordinates within the system (see Figure 6.8). The side walls, face sheet, and rear plate are modeled using their constituent, temperature-dependent thermal conductivities (given in Appendix A for SS304). The approach taken for the saturated wick and vapor thermal conductivity is described below. 


\section{4 (a) Vapor Thermal Conductivity}

An expression for the effective vapor conductivity, $k_{e f f}$, as a function of the axial temperature drop along the vapor core, $\Delta T_{V}$ can be written using Fourier's law [15];

$$
k_{e f f}=\frac{\bar{Q} L_{t}}{\Delta T_{V} \bar{A}_{V}}
$$

where the total length, $L_{t}$, is the sum of the evaporator $\left(L_{e}\right)$ and condenser $\left(L_{c}\right)$ lengths given by $L_{e}=R_{L E}\left(\frac{\pi}{2}-\varphi\right) \cos \varphi$ and $L_{c}=L_{\text {flat }} \cos \varphi . \quad \bar{Q}$ is the length-averaged cumulative heat in the vapor, and $\bar{A}_{V}$, the average cross-sectional area of the vapor for the diverging wedge design, are given by;

$$
\begin{gathered}
\bar{Q}=\frac{1}{L_{t}} \int_{0}^{L_{t}} Q(x) d x \\
\bar{A}_{V}=\frac{1}{L_{t}} \int_{0}^{L_{t}} A_{V}(x) d x
\end{gathered}
$$

where $Q(x)$ and $A_{V}(x)$ are the position-dependent cumulative heat and vapor crosssectional area as a function of $x$ (see Figure 4.23). The former is approximated as a piecewise linear function to approximate the imposed experimental heat flux;

$$
Q(x)= \begin{cases}\frac{x}{L_{e}} Q_{\max } & 0 \leq x<L_{e} \\ \frac{L_{t}-x}{L_{c}} Q_{\max } & L_{e} \leq x \leq L_{t}\end{cases}
$$

For the diverging wedge geometry; 


$$
A_{V}(x)=2 W H_{V}(x)
$$

where the vapor core height has been previously given as $H_{V}(x)$;

$$
\begin{aligned}
H_{V}(x)=R_{L E} & {\left[\cos \varphi-\left(\frac{\pi}{2}-\varphi\right) \sin \varphi\right]-\left(b_{\text {wall }}+b_{\text {wick }}\right) \sec \varphi } \\
& +x \tan \varphi
\end{aligned}
$$

An expression for $\Delta T_{V}$ is required to solve equation (6.5). Assuming saturation conditions, the integrated form of the Clausius Clapeyron equation [108] provides an expression for the pressure difference over the length of the vapor core;

$$
\begin{aligned}
\Delta P_{V}=P_{V}(x & \left.=L_{t}\right) \operatorname{Exp}\left[-\frac{M \lambda}{R}\left(\frac{1}{T_{V}(x=0)}-\frac{1}{T_{V}\left(x=L_{\mathrm{t}}\right)}\right)\right] \\
& -P_{V}\left(x=L_{t}\right)
\end{aligned}
$$

where $R$ is the universal gas constant $(8.314 \mathrm{~J} / \mathrm{mol} \cdot \mathrm{K}), M$ is the molar mass of the fluid, $\lambda$ is the latent heat, and where $T_{V}$ and $P_{V}$ are the vapor temperature and pressure at the subscript-specified locations. Solving for $\Delta T_{V}\left(=T_{V, x=0}-T_{V, x=L_{t}}\right)$ and approximating

$$
\begin{gathered}
T_{V}(x=0) T_{V}\left(x=L_{t}\right) \approx T_{V}^{2} \text { and } \ln \left[\frac{\Delta P_{V}}{P_{V}\left(x=L_{t}\right)}+1\right] \approx \frac{\Delta P_{V}}{P_{V}\left(x=L_{t}\right)} \text { gives; } \\
\Delta T_{V}=\frac{T_{V}^{2} R}{M \lambda} \frac{\Delta P_{V}}{P_{V}\left(x=L_{t}\right)}
\end{gathered}
$$

An expression is now needed for the vapor core pressure drop, $\Delta P_{V}$, appearing in equation (6.12). Its differential form was derived in Chapter 4 (equation (4.54)); 


$$
\begin{array}{r}
d P_{V}=-\frac{\beta}{\rho_{V} A_{V}{ }^{2} \lambda^{2}}\left(2 Q d Q-\frac{Q^{2}}{\rho_{V}} d \rho_{V}-\frac{Q^{2}}{A_{V}} d A_{V}\right) \\
-\frac{2 \mu_{V} f \operatorname{Re}_{V}\left(W+2 H_{V}\right)^{2} \cos \varphi}{\lambda \rho_{V} A_{V}{ }^{3}} Q d x
\end{array}
$$

$\beta$ is a momentum correction factor (taken as $4 / 3$ for laminar flow), $\rho_{V}$ and $\mu_{V}$ are the vapor density and viscosity, $W$ is the vapor core width, $\varphi$ is the wedge half angle, $f$ is the friction factor, and $\operatorname{Re}_{V}$ is the vapor Reynolds number. Assuming operation well below the sonic limit, the vapor can be taken as incompressible $\left(d \rho_{V}=0\right)$ so that integration yields;

$$
\Delta P_{V}=\frac{2}{\rho_{V} \lambda} \cdot \int_{L_{t}}^{0}\left(\frac{\beta W \tan \varphi}{\lambda A_{V}^{3}} Q^{2}-\frac{\mu_{V} f \operatorname{Re}_{V}\left(W+2 H_{V}\right)^{2} \cos \varphi}{A_{V}^{3}} Q\right) d x
$$

Note that the $d Q$ term drops out as a result of the heat balance imposed over the length of the system (i.e. the static pressure loss associated with the $d Q$ term during evaporation is recovered upon condensation). The discontinuity at $x=L_{e}$ in the expression for $Q(x)$ prevents an analytical solution of the integral so numerical methods must be used. The software package Mathematica (using the NSolve command) was used for its solution.

Figure 6.7 shows the solved effective thermal conductivity of water vapor as a function of $Q_{\max }$ for different vapor saturation temperatures. Water's thermodynamic properties used in the solution are given in Appendix B. The computed $k_{e f f}$ values are several orders of magnitude higher than any known solid: for comparison, the thermal conductivities of copper and isotropic pure diamond are $395 \mathrm{~W} / \mathrm{mK}$ [50], and 4,000W/mK 
[131], respectively. While the plot shows an increasing $k_{\text {eff }}$ with temperature, its value is so high that its temperature variation has little discernible effect in model predictions (this is demonstrated in $\$ 6.5)$.

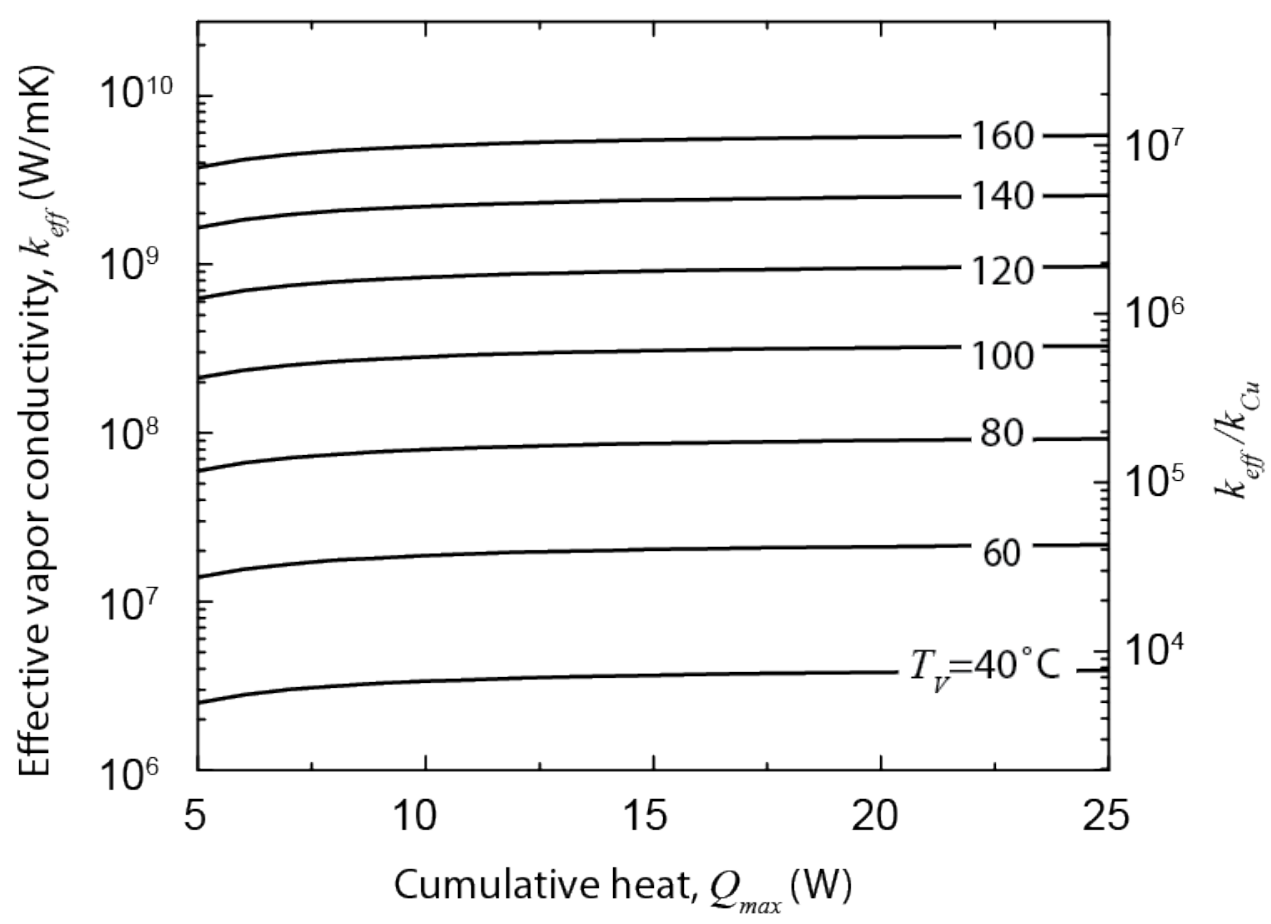

Figure 6.7. The effective thermal conductivity of the vapor as a function of $Q_{\text {max }}$. The right ordinate normalizes $k_{\text {eff }}$ by the room temperature thermal conductivity of copper $\left(k_{C u} \approx\right.$ $400 \mathrm{~W} / \mathrm{mK})$.

\section{4(b) Wick thermal conductivity}

The effective thermal conductivity of a water-saturated wick can be calculated using the multi-layer screen model developed by Peterson [72]. This leads to a wick conductivity that depends on wick porosity and thermal conductivity of the wick and working fluid; 


$$
k_{w i c k}=k_{L} \frac{k_{L}+k_{S s}-(1-\varepsilon)\left(k_{L}-k_{S}\right)}{k_{L}+k_{S}+(1-\varepsilon)\left(k_{L}-k_{S}\right)}
$$

where $k_{L}$ is the thermal conductivity of the liquid, $k_{S}$ is the thermal conductivity of the solid wire from which the screen is made, and $\varepsilon$ is the porosity (defined in equation (6.1)). For the unsaturated wick, a value of $k_{L}=0.03 \mathrm{~W} / \mathrm{mK}$ [108] was used which is close to air at room temperature.

\section{4(c) Boundary Conditions}

Boundary conditions are shown in Figure 6.8. The heat flux condition imposed over the curved tip was;

$$
q_{i n}=\frac{T_{h}-T_{w}}{R_{h}}
$$

where $q_{i n}$ is the net heat flux in the direction normal to the curved surface. $T_{h}$ and $T_{w}$ are the heater and wall temperature, respectively, and $R_{h}$ is the conduction resistance of the heater-wall interface. The flat portion of the face sheet was cooled by free convection to the ambient (taken to be at temperature $T_{\infty}=23^{\circ} \mathrm{C}$ ) with heat transfer coefficient $h_{\text {cool }}=$ $10 \mathrm{~W} / \mathrm{m}^{2} \mathrm{~K}$. All other surfaces were treated adiabatic. 


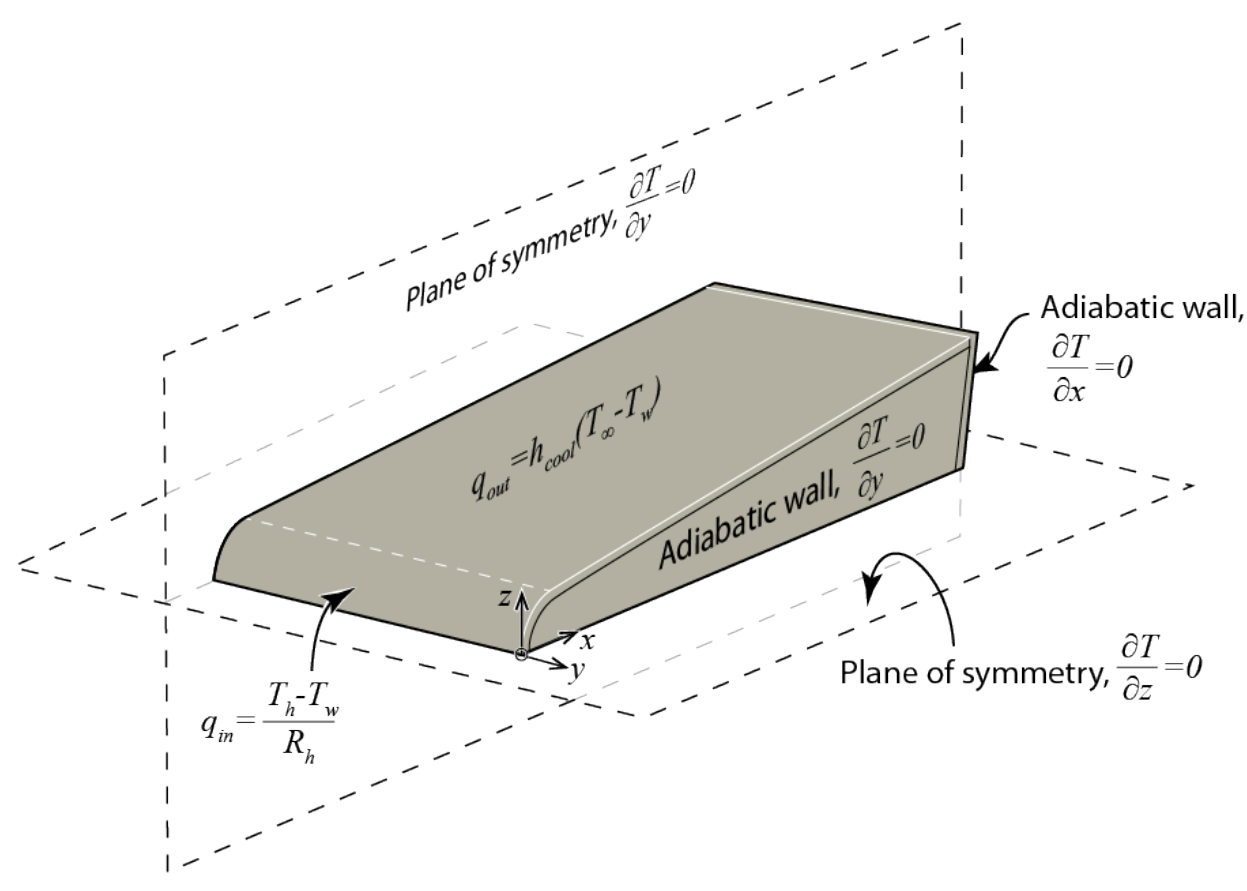

Figure 6.8. Boundary conditions of the quarter symmetry model.

The finite element thermal model was solved using SolidWorks ${ }^{\circledR}$ Simulation running an iterative Fourier Finite Element (FFE) solver algorithm. Figure 6.9 shows the meshed quarter-symmetry model evaluated for the current study. Trial solutions using finer meshes gave converged solutions that were within $2 \%$ (over all nodal points) than the mesh shown in the figure. Convergence criteria was specified to be within $0.1 \%$. 


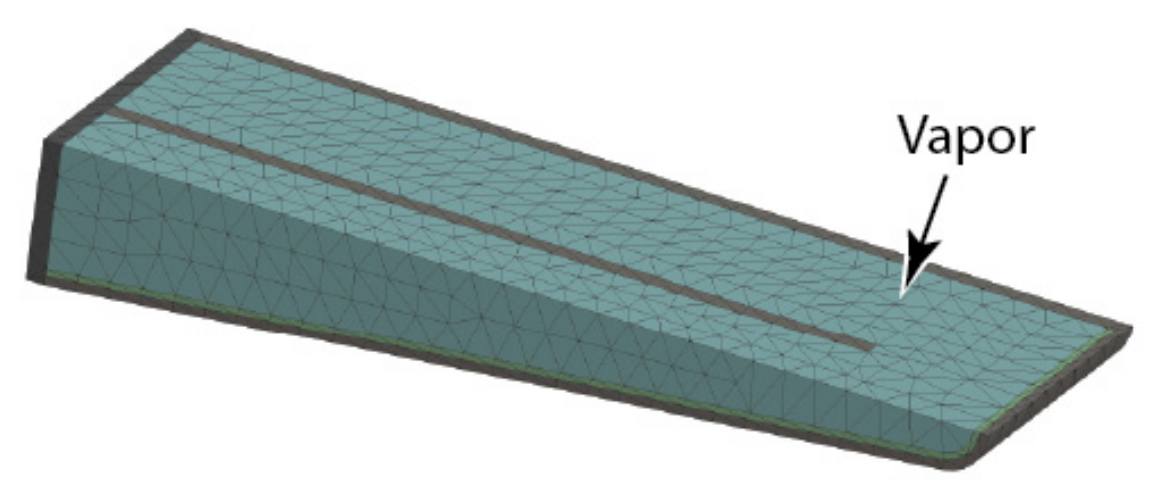

Figure 6.9. Isometric view of quarter-symmetry model showing the mesh.

The model geometry is identical to the as-fabricated, low-temperature structures tested except that the fill tube on the rear face was not included in the models. All contact points between components were given a thermal resistance of zero. All of the geometric values are listed in Table 6.1.

In order to acquire a value for the interfacial resistance $R_{h}$ between the heater and wall, a finite element model of the evacuated system was solved with an initial guess, and the resulting output was compared with the experimental temperature data of the evacuated system. This process was iterated (changing $R_{h}$ ) until good agreement between the simulation and experimental data were achieved. The converged upon resistance $\left(R_{h}=\right.$ $0.014 \mathrm{~m}^{2} \mathrm{~K} / \mathrm{W}$ ) was then used in the model with the vapor core and compared to the experimental results of the charged system.

Earlier it was hypothesized that while the analytical vapor transport theory predicted thermal conductivities on the order of $\mathrm{MW} / \mathrm{mK}$, these values are so high that its variation 
will not show any discernible difference in the temperature predictions of the finite element

model. A check of the model was run with values of $k_{\text {eff }}=3,000 \mathrm{~W} / \mathrm{mK}$ and $k_{\text {eff }}=$ $10^{6} \mathrm{~W} / \mathrm{mK}$ and the predicted temperatures were found not to vary by more than $0.62 \%$. The lower value provided slightly faster convergence times and was used in subsequent calculations.

\subsection{Simulation results and discussion}

Figure 6.10 compares the simulated surface temperature as a function of axial position and compares them to the experimental data at steady state for applied power levels of 5,15 , and $25 \mathrm{~W}$. Surface temperatures are taken along the line profiles showed in the inset of the plot labeled I(a). The plots of Column I show good agreement (within 8\%) between the model and surface temperature data of the evacuated system at all power levels. Column II compares the simulation and experimental data taken on the charged leading edge with the same thermal resistance. Overall, good agreement is found to exist between the simulation temperatures and thermocouple readings at the tip, not deviating by more than $8.1 \%$ at $5 \mathrm{~W}$ and less than $2.5 \%$ at the 10 and $15 \mathrm{~W}$ levels. The nearly isothermal operating temperatures aft of the tip region show very good agreement between the simulation and IR readings. 
I. Evacuated (no working fluid)
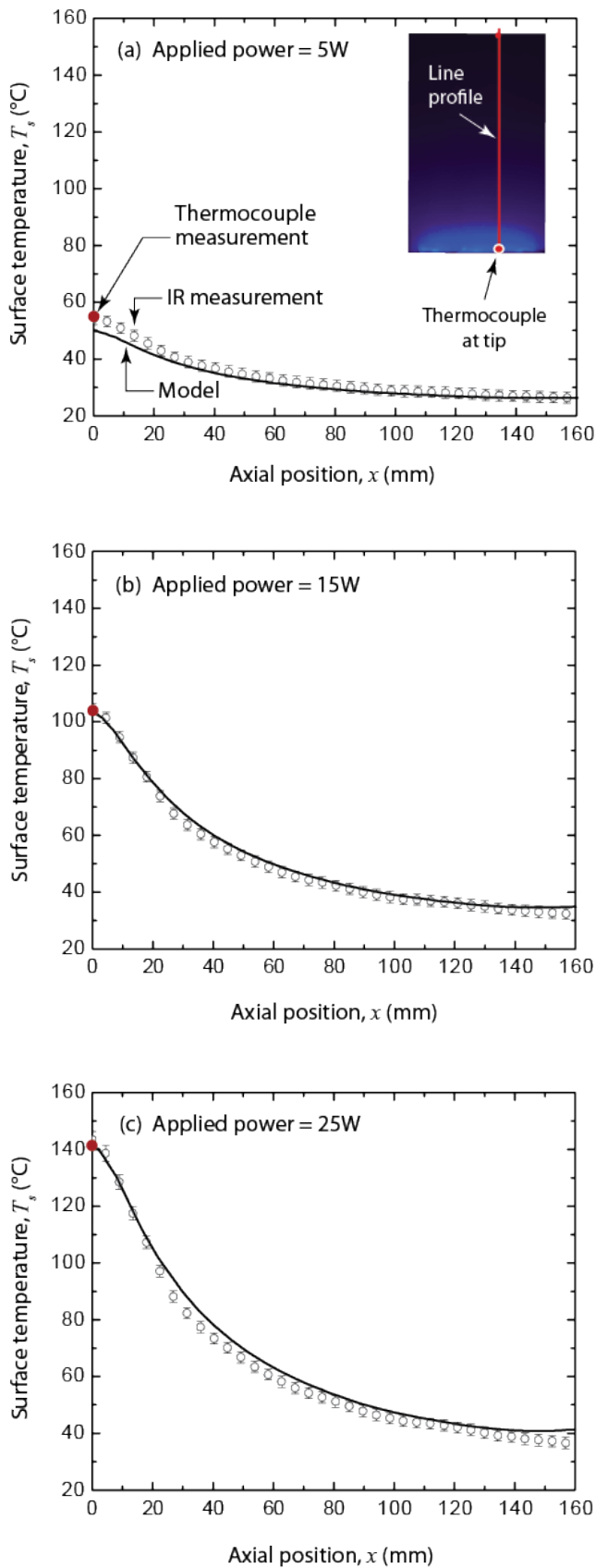

II. Charged (working fluid)
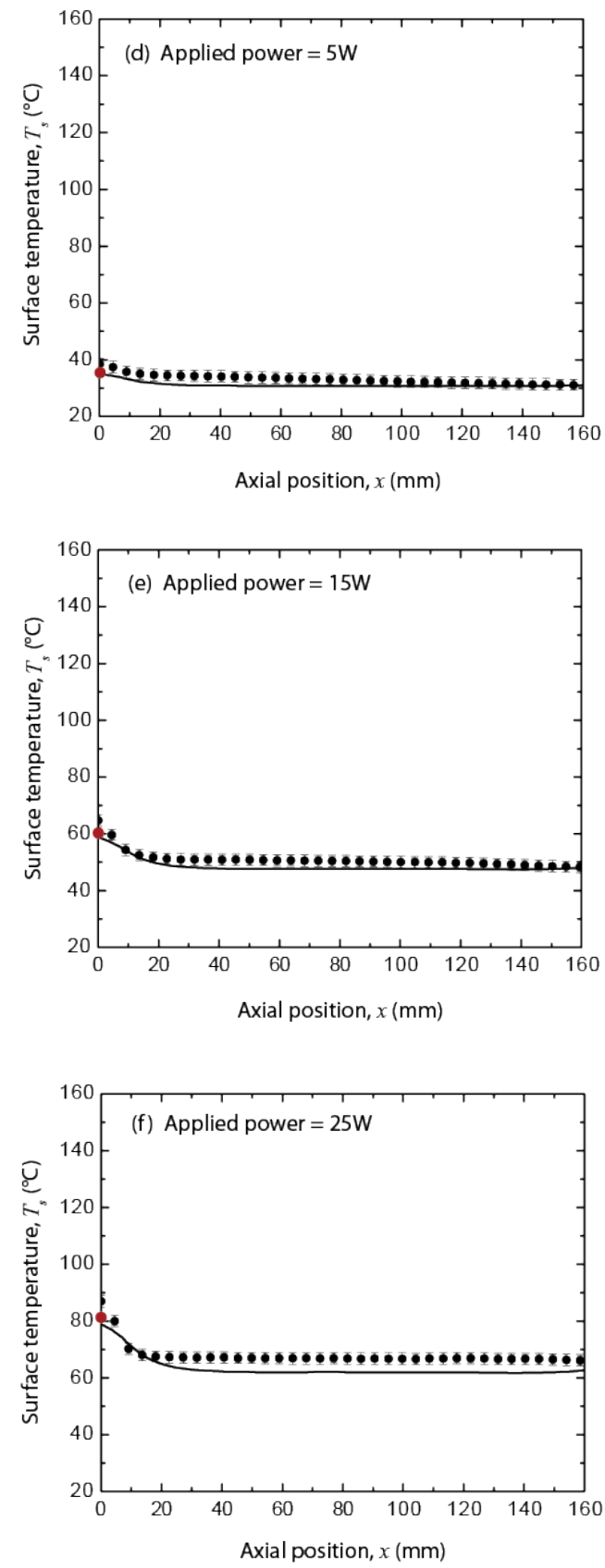

Figure 6.10. Comparison of the simulated and experimental line profile surface measurements of (I) the evacuated and (II) charged leading edge at applied power levels of 5, 15, and 25W. The inset in I(a) shows the location of the line profile. The "•" data point is the tip thermocouple temperature reading. 
The surface temperature difference over the axial length of the test articles is plotted in Figure 6.11. The evacuated sample has a tip-to-rear temperature difference of over $100^{\circ} \mathrm{C}$ at $25 \mathrm{~W}$ and varies nearly linearly with increasing power. The charged sample experiences less than a $20^{\circ} \mathrm{C}$ difference at $25 \mathrm{~W}$ and trends toward a constant value with increasing power, suggesting that it is operating below its heat transport limits.

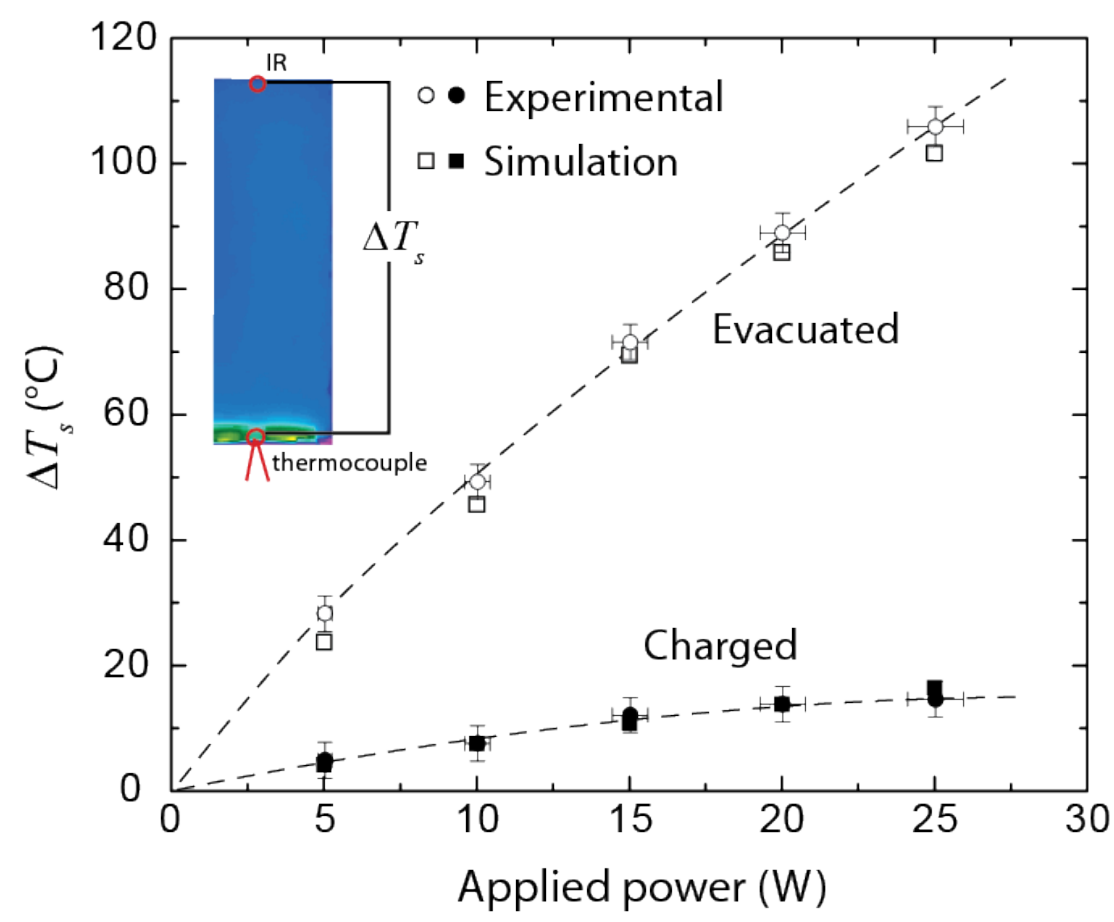

Figure 6.11. Steady state comparison of the predicted surface temperature difference to experimental data. The difference is taken from the thermocouple measurement at the tip to the rear edge IR measurement as shown in the inset. The dashed lines are best fits to the data.

Figure 6.12 compares the operating condition (surface temperature and applied power) against the predicted capillary limit for areal evaporator fractions of $F=1.0,0.3$, and 0.05 , although the theoretical maximum active areal fraction for evaporation from this 
wick is $F_{\max }=0.3$ (given by equation (4.43)). The plotted heat fluxes at 5, 15, and 25W were obtained from the F.E. simulation. Restricted by the heater's maximum operating power, all tests were conducted below the $F=0.3$ capillary limit. No tip dryout was observed.

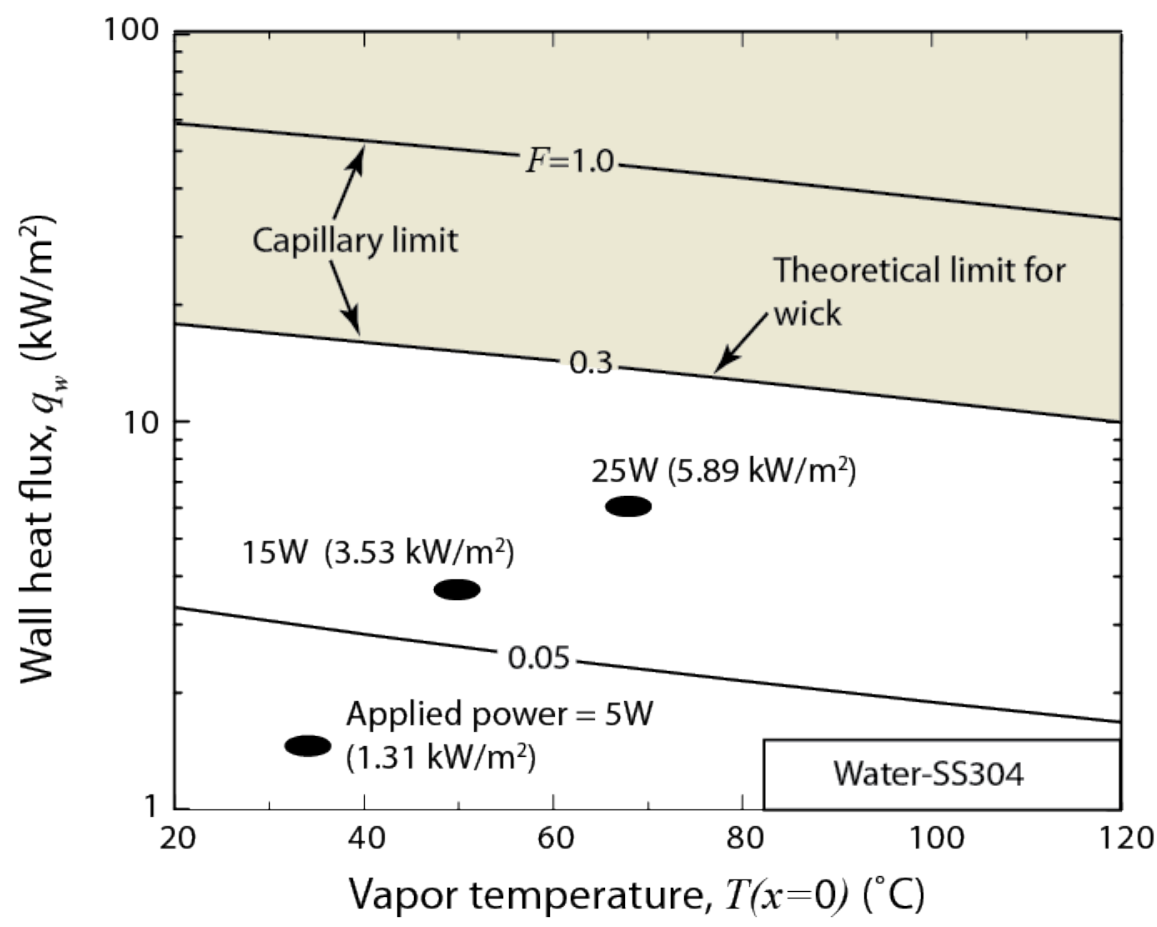

Figure 6.12. Operating limits for the water-SS304 leading edge heat spreader. The sonic and boiling, and yielding limits are not shown because they are well above the plot's range.

The design model presented in Chapter 3 assumed the wall surface temperature of the condenser to be approximately equal to the isothermal vapor temperature. Experimental validation of this approximation is provided in Figure 6.13 which plots the IR temperature measurements and vapor core pressure readings taken by the transducer. The Clausius Clapeyron equation was used to correlate the left and right ordinate axes. The plot shows a 
wall surface temperature operating at a slightly lower temperature than the saturation temperature of the vapor, providing a cool, condensing surface which pulls the latent heat out of the vapor.

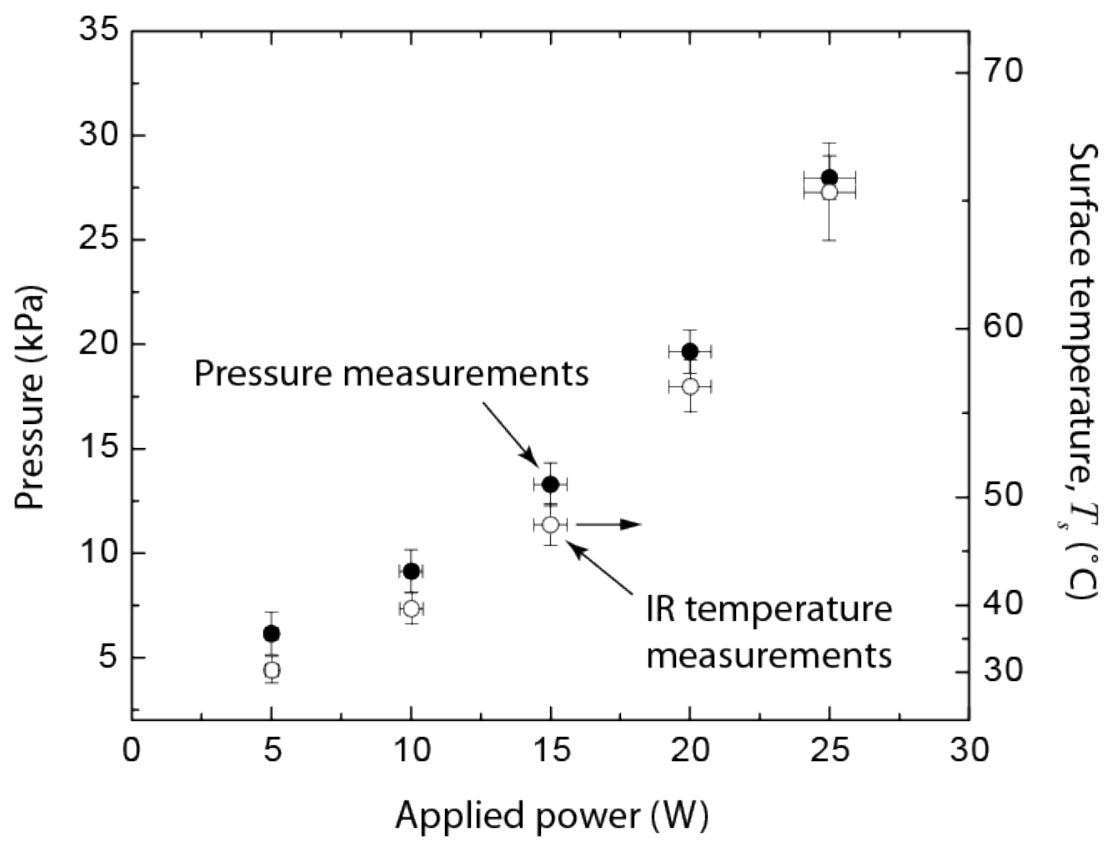

Figure 6.13. Comparison of isothermal surface temperature (taken by the IR camera) and vapor pressure measurements correlated by the Clausius-Clapeyron equation. The IR temperature measurements correspond to the right ordinate.

\subsection{Summary}

This chapter has experimentally demonstrated the ability of heat pipe leading edges to lower the tip temperatures by a localized heat input and the predictive capability of the finite element simulation for low temperature testing. Because of evaporation and condensation events, a working fluid must exist as a two-phase (liquid and vapor) system for a heat pipe to 
successfully operate. The critical temperature of water is $374^{\circ} \mathrm{C}$ [28], well below the temperatures experienced at the leading edges during hypersonic flight, preventing its use for that application. In the next chapter, the fabrication of a high temperature leading edge is described which was tested at temperatures close to those for low altitude, hypersonic flight. 


\section{Chapter 7. High temperature concept testing}

Having demonstrated, through analytical modeling, F.E. analysis, and low temperature experiments, the potential for a leading edge heat spreader, a high temperature IN718-sodium system suitable for Mach 5 flight (at an altitude of $24.5 \mathrm{~km}$ ) was explored in similar fashion. Design parameters (length and wall thickness) were chosen to lie within the operational region of the design map formulated in Chapter 4 and a route for fabrication developed. High heat flux testing was conducted using an oxy-acetylene welding torch. The charged heat pipe leading edge system exhibited a significantly decreased tip temperature compared to an identical but evacuated test article that contained no working fluid. Nonetheless, the fully charged system did not exhibit perfect isothermal operation because the device operated in a state where self-diffusion of vapor molecules, rather than bulk convection, dominated vapor (and thermal) transport in the vessel. 


\subsection{Leading edge design and fabrication}

IN718 was chosen for the case material due to it high strength at elevated temperatures, good weldability, and oxidation resistance. Sodium was chosen for the working fluid since it has a high latent heat of vaporization and is chemically compatible with IN718 [89], [132]. A schematic of the IN-718 / Na system is shown in Figure 7.1 (with rear face sheet removed). The test represented a single cell of an I-core leading edge having a low vapor flow resistance in the longitudinal (fore-aft) direction. A contiguous nickel foam wick lined the upper and lower interior surface to provide an uninterrupted liquid flow path between the flat, condensing region and the evaporating region along the rounded curvature of the tip. The leading edge radius, $R_{L E}$, and wedge half angle, $\varphi$, were set to $3 \mathrm{~mm}$ and $6^{\circ}$, respectively. The length of the flat section, $L_{\text {flat }}$, and wall thickness, $b_{\text {wall }}$, were selected using the Mach 5 design map presented in Chapter 3 and shown again here as Figure 7.2. The map shows a large design space in which tip stresses remain below the alloy's temperature-dependent yield strength. $L_{\text {flat }}$ and $b_{\text {wall }}$ were taken to be $82.3 \mathrm{~mm}$ and $1.6 \mathrm{~mm}$, respectively, which was expected to exhibit a maximum temperature of $-760^{\circ} \mathrm{C}$ at the tip and surfaces aft of the tip operating isothermally at $-710^{\circ} \mathrm{C}$. All the geometric parameters are summarized in Table 6.1. 


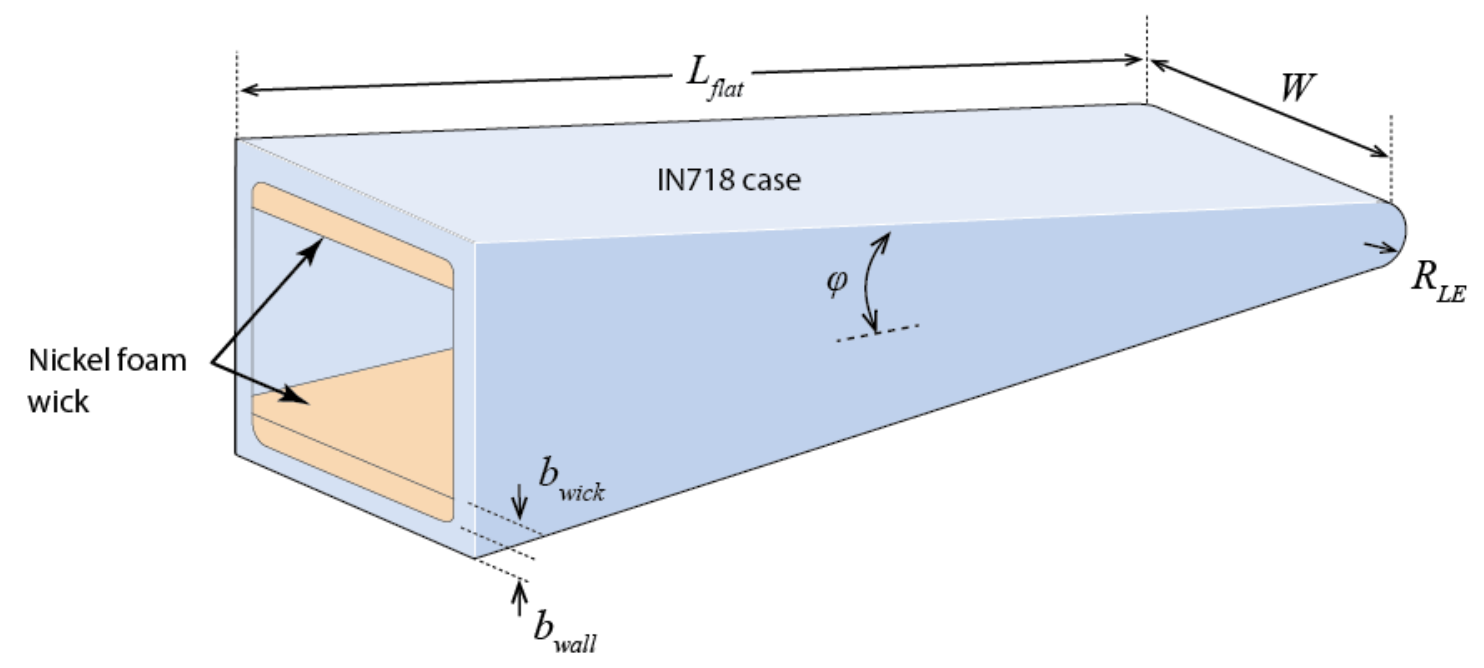

Figure 7.1. Schematic of the IN718 / Na system with relevant design parameters. Aerodynamic constraints set $R_{L E}=3 \mathrm{~mm}$ and $\varphi=6^{\circ}$. 


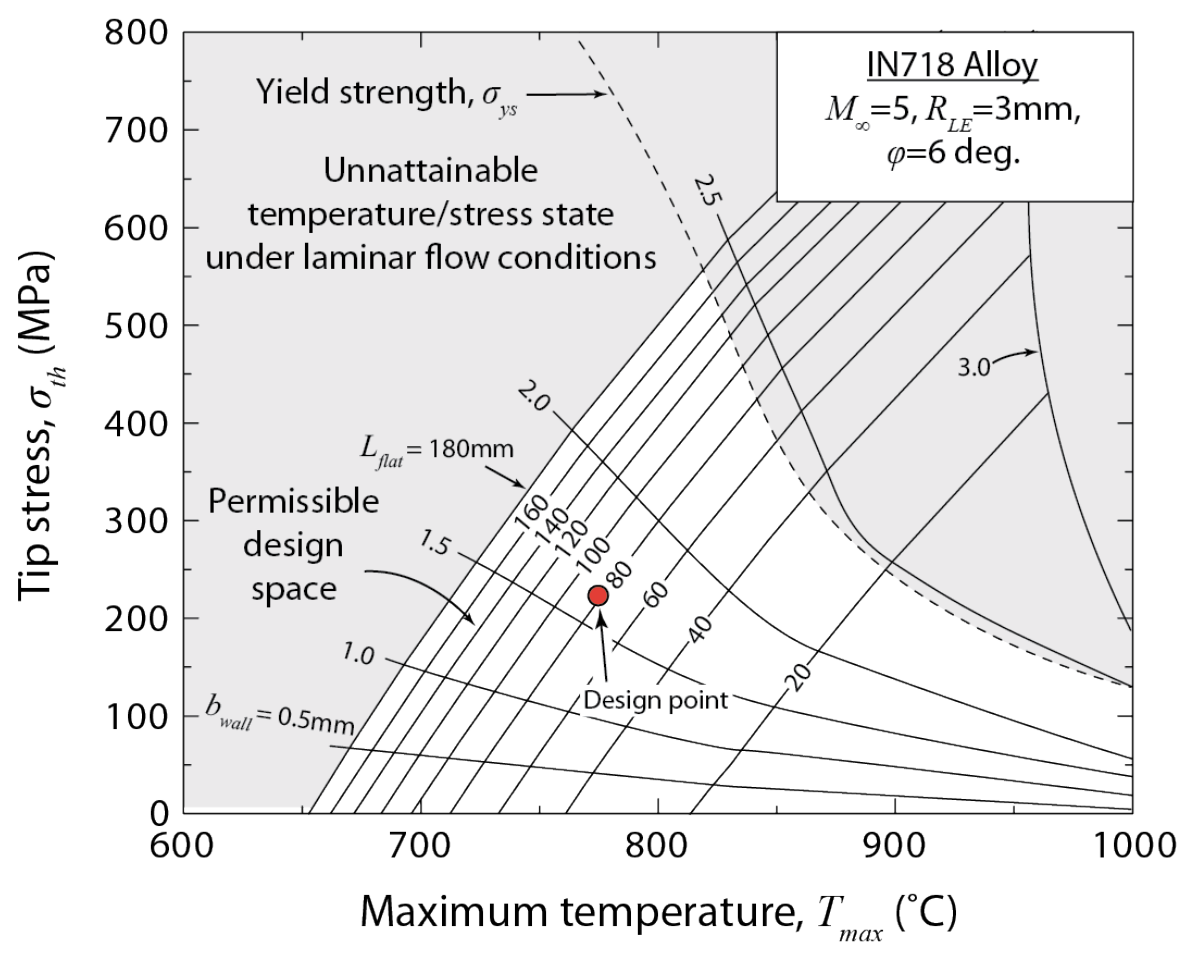

Figure 7.2. IN718 / Na design map for Mach 5 flight. 
Table 7.1. Relevant parameters for the high temperature leading edge heat spreader.

Working Fluid (See Appendix B for thermo-physical property data)

Working fluid type

Sodium

\section{Case Design (See Appendix A for thermo-mechanical property data)}

Case material

Length of flat surface, $L_{\text {flat }}$

Half angle, $\varphi$

Leading edge radius, $R_{L E}$

Wall thickness, $b_{\text {wall }}$

Width, $W$

\section{Wick Design}

Wick material

Wick thickness, $b_{\text {wick }}$

Compressed wick porosity, $\varepsilon$

Pore radius, $R_{P}$
IN-718

$82.3 \mathrm{~mm}$

$6^{\circ}$

$3.0 \mathrm{~mm}$

$1.59 \mathrm{~mm}$

$21.2 \mathrm{~mm}$

Open cell Ni foam

$0.71 \mathrm{~mm}$

$93.4 \%$

$225 \mu \mathrm{m}$

The fabrication sequence is illustrated in Figure 7.3. The case exterior surface was electro-discharge machine (EDM) wire-cut from annealed an IN-718 block (Special Metals ${ }^{\oplus}$, Huntington, WV) and further machined using a ram Electro-Discharge Machining (EDM) process (Quality Machine Works ${ }^{\oplus}$ Waynesboro, VA) which hollowed its interior to the design wall thickness. The sacrificial graphite electrodes of the ram EDM process leave a heavy carbon residue on the machined surfaces; this was removed using a mixture of acetone, xylene, and toluene.

The wick was a stochastic nickel foam (Novamet ${ }^{\oplus}$ INCOFOAM $^{\mathrm{TM}}$ ) with an asreceived $97 \%$ porosity, $1.6 \mathrm{~mm}$ thickness, and $450 \mu \mathrm{m}$ average pore diameter [133] which was confirmed by optical microscopy. To fit within the curvature of the tip, the foam was 
compressed in the normal direction to $b_{w i c k}=0.71 \mathrm{~mm}$ using platens fixtured in a press brake. Queheillalt et al. [84] showed via scanning electron micrographs and the rising meniscus experimental method that the compression ratio (compressed thickness to uncompressed thickness) of the open cell nickel foam closely follows the constriction ratio (compressed pore diameter to uncompressed pore diameter) of the effective pore diameter. Therefore, the compression ratio used here, -0.5 , yielded a pore diameter of half the uncompressed value, or $225 \mu \mathrm{m}$.

A high temperature sinter process was used to ensure good contact between the foam and IN718 case. During sintering, high purity alumina pellets $(3 \mathrm{~mm}$ diameter, Sigma Aldrich $^{\odot}$ ) were backfilled on top of the foam, the pellet stack taking on the shape of the wedge to hold the foam against the interior wall surfaces. A $25 \mathrm{~g}$ weight placed on top of the stack provided sufficient sintering force. The sample was placed in a furnace along with titanium getter, heated to $550^{\circ} \mathrm{C}$ at $10^{\circ} \mathrm{C} / \mathrm{min}$ and held for $10 \mathrm{~min}$ to burn off any organic residue at a pressure of $10^{-4}$ Torr. The sample was then heated to $1200^{\circ} \mathrm{C}$ at $10^{\circ} \mathrm{C} / \mathrm{min}$ and held for 60 minutes at $10^{-4}$ Torr before cooling to ambient at $-10^{\circ} \mathrm{C} / \mathrm{min}$. Figure 7.4 is a scanning electron micrograph of a test coupon showing the cross-section of the bonded wick. There is excellent metallurgy bonding between the foam ligaments and the IN718 substrate, indicating the formation of a low thermal resistance pathway between with the wick and case. After bonding, the pellets were removed and a monolithic IN718 rear face plate with fill port was electron beam welded to the sample's open end at its edges under an ambient 
pressure of $10^{-6}$ Torr, Figure 7.5. After welding, the hermicity of the sample was helium leak checked to less than $1.5 \times 10^{-8} \mathrm{~Pa}-\mathrm{m}^{3} / \mathrm{s}$ using a Leybold ${ }^{\oplus}$ UL200 leak detector.

Unlike previously reported charging methods which heated the alkali metal working fluid for a liquid-state backfill [72], [73], [74], a solid-state technique was developed which allowed handling of the highly reactive alkali in its more stable state. The sample was charged with sodium (99.9\% purity packed under mineral oil, Sigma Aldrich ${ }^{\oplus}$ ) under a nitrogen cover atmosphere with measured oxygen levels of less than $10 \mathrm{ppm}$. $6.510 \mathrm{~g}$ of room-temperature sodium pellets were placed in the sample, sufficient to fully saturate the void volume in the foam at $700^{\circ} \mathrm{C}$ with an $18.7 \mathrm{wt} \%$ overcharge. A solid IN-718 cylindrical plug having shallow surface channels extending between its opposing ends was push fit into the fill port and butted against a shallow ledge machined into its inner wall. A ball valve (HyLok 105 series) placed on the fill tube temporarily sealed the sample so it could be removed from the nitrogen atmosphere. The sample was then connected to a vacuum turbo pump and evacuated to a final vacuum level of $10^{-6}$ Torr. The plug's surface channels allowed the trapped nitrogen gas to flow around the plug and into the line. A $2 \mathrm{~kW} \mathrm{Nd:YAG}$ laser was used to permanently seal the sample by welding the fill tube to the plug before removing the port valve. In order to distribute the sodium through the wick, the sample was heated to $718^{\circ} \mathrm{C}$ and held for 8 hours at $10^{-4} \mathrm{Torr}$, cooled to $621^{\circ} \mathrm{C}$ and held for 8 hours, and then furnace cooled. A second sample without sodium was identically fabricated and sealed to serve as a test baseline. 

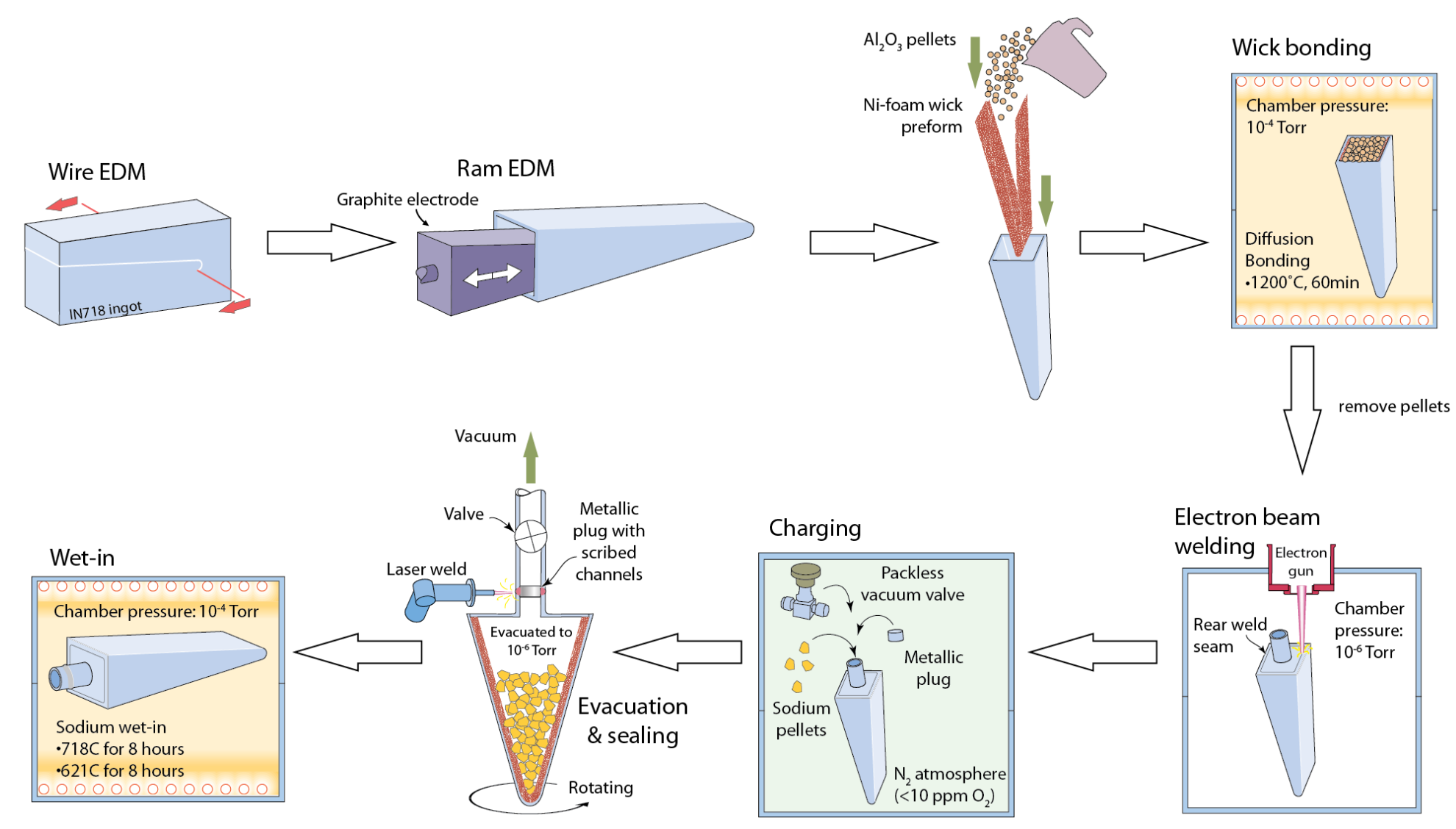

Figure 7.3. Fabrication and assembly process for the high temperature leading edge using the solid-Na charging procedure. 


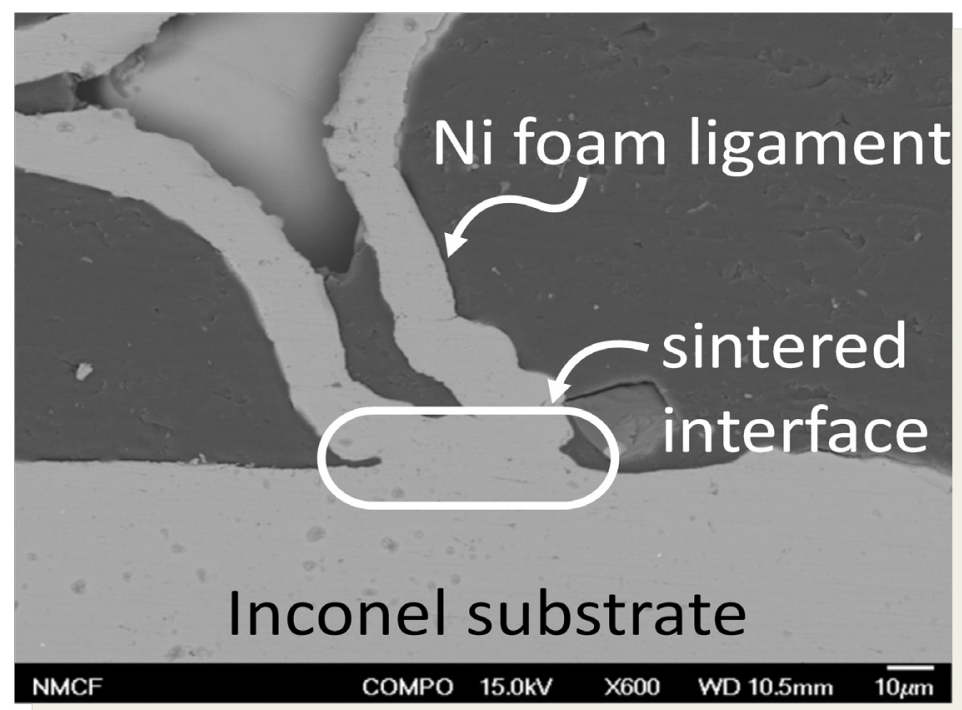

Figure 7.4. Scanning electron micrograph of the sintered foam-IN718 interface.

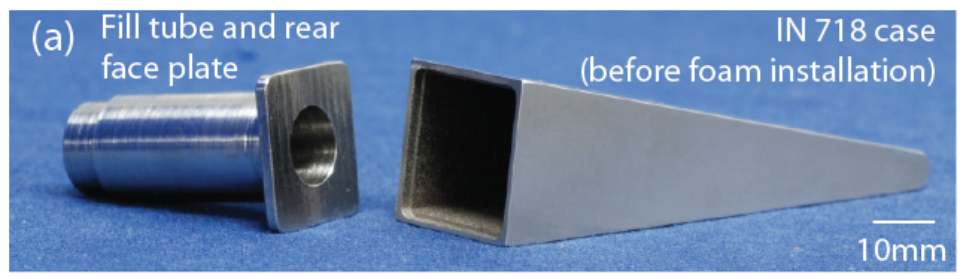

(b)
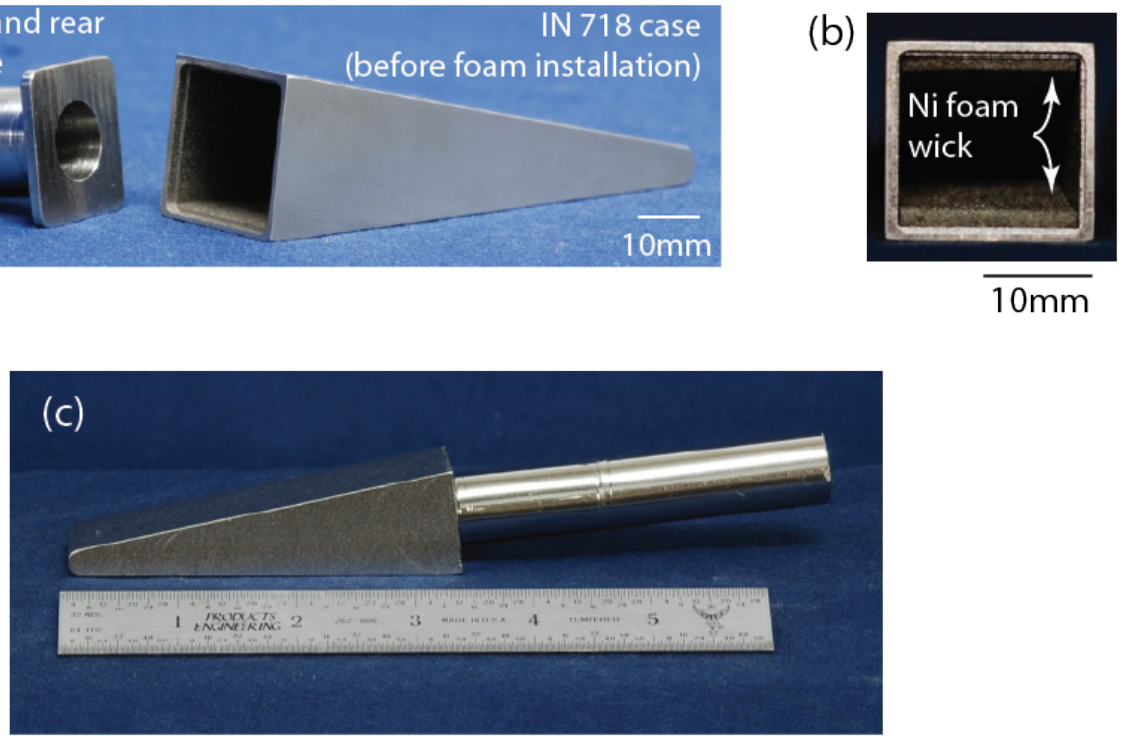

Figure 7.5. Photograph of the fabricated high temperature leading edge (a) prior to insertion of the wick and (b) after diffusion bonding of the wick. The completed test article is shown in (c). 


\subsection{Test setup and instrumentation}

The test setup shown in Figure 7.6 was used to evaluate the performance of the IN718-sodium system under an impinging heat flux comparable to that of Mach 5 operation at an altitude of $24.5 \mathrm{~km}$. An oxy-acetylene welding torch was mounted to a linear actuator which allowed its standoff distance from the curved sample tip to be adjusted to within $\pm 1 \mathrm{~mm}$. The sample was firmly suspended by its fill tube within $\pm 0.5^{\circ}$ of the horizontal. A refractory $\mathrm{Al}_{2} \mathrm{O}_{3}-\mathrm{SiO}_{2}$ firebrick shield was fitted around the sample to insulate its sides. The sample's tip extended $2.6 \mathrm{~mm}$ through a slot in the shield's front face which exposed just the curved tip to the direct impingement of combustion gases. An insulating fibrous aluminasilica blanket (Zircar ${ }^{\circledR}$ Type ASB-2600) was fit into any voids between the shield and sample as well as adhered against the sample rear face plate and fill tube.

Steady state surface temperature measurements were taken using an infrared (IR) camera (Model A325, FLIR ${ }^{\oplus}$ Systems Inc., Boston, MA, 7.5 to $13 \mu \mathrm{m}$ spectral range) mounted above the sample with its field of view set orthogonal to the upper surface. The camera was factory calibrated for operation within the 200 to $1200^{\circ} \mathrm{C}$ range with an error of $\pm 2 \%$ of the reading. The imaged surface was painted with a high temperature, high emissivity paint (Aremco ${ }^{\oplus} \mathrm{HiE}^{-C o a t}{ }^{\mathrm{TM}} 840-\mathrm{M}$ ) to set the surface emissivity to 0.95 . The internal emissivity correction of the infrared camera was adjusted to this value. A type-K thermocouple was mounted at the middle of the flat sample surface (at $L_{\text {flat }} / 2$ ) to check the camera's readings. Differences in the thermocouple and camera measurements were found 
to be within camera error. It should be noted that radiation from the hot gas which envelopes the sample tip will interfere with IR temperature readings in this region. The extent of this interference and its effect on measurement accuracy is assessed with the finite element model presented later. 
(a)

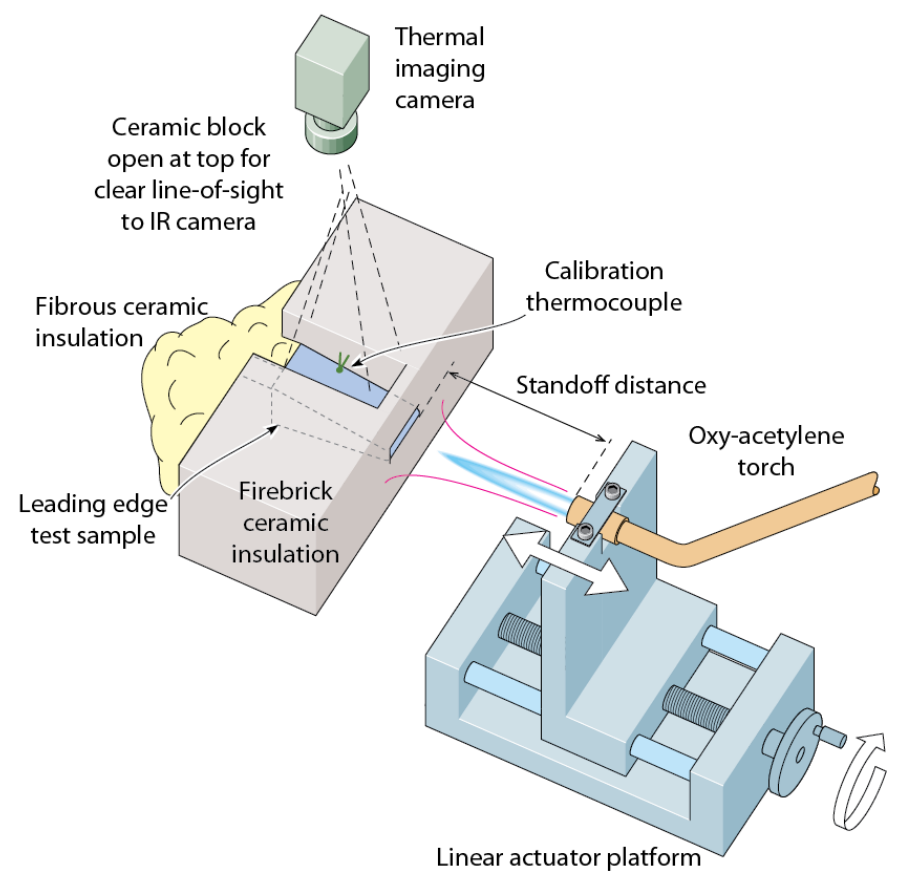

(b)

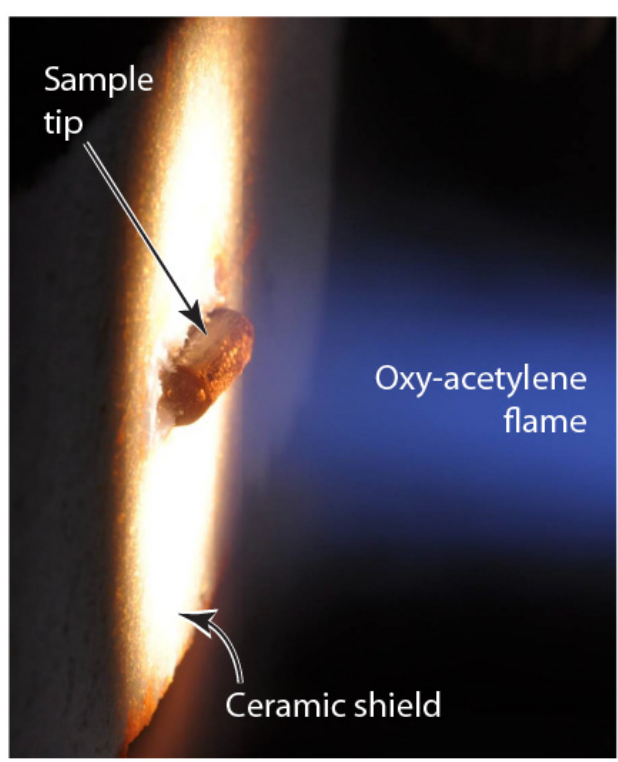

Figure 7.6. (a) Setup for the high heat flux experiments, and (b) photograph of the sample tip during testing. 


\subsection{Experimental results}

Steady state axial temperature profiles taken by the IR camera along the central axis of the top surface of both the sodium-charged and evacuated test structures for three standoff distances are shown in Figure 7.7. Steady state was assumed once the time-average of 100 consecutive temperature readings, taken every $1 \mathrm{~s}$, did not change by more than $2 \%$ over a period of 10 minutes. Temperature readings in the region masked by the ceramic firebrick shield could not be made and are not shown in the plots. As the standoff distance decreased (torch brought closer to the test article), the wedge tip temperature rose. While not operating perfectly isothermal, the charged system has a smaller thermal gradient across its condenser length than the evacuated sample. The charged system's tip temperatures are more than $15 \%$ lower over each of the three standoff distances. The lower tip temperature and higher condenser temperature of the charged test article suggests a more effective heat transfer mechanism than that of solid conduction alone, as is the case with the evacuated sample. 

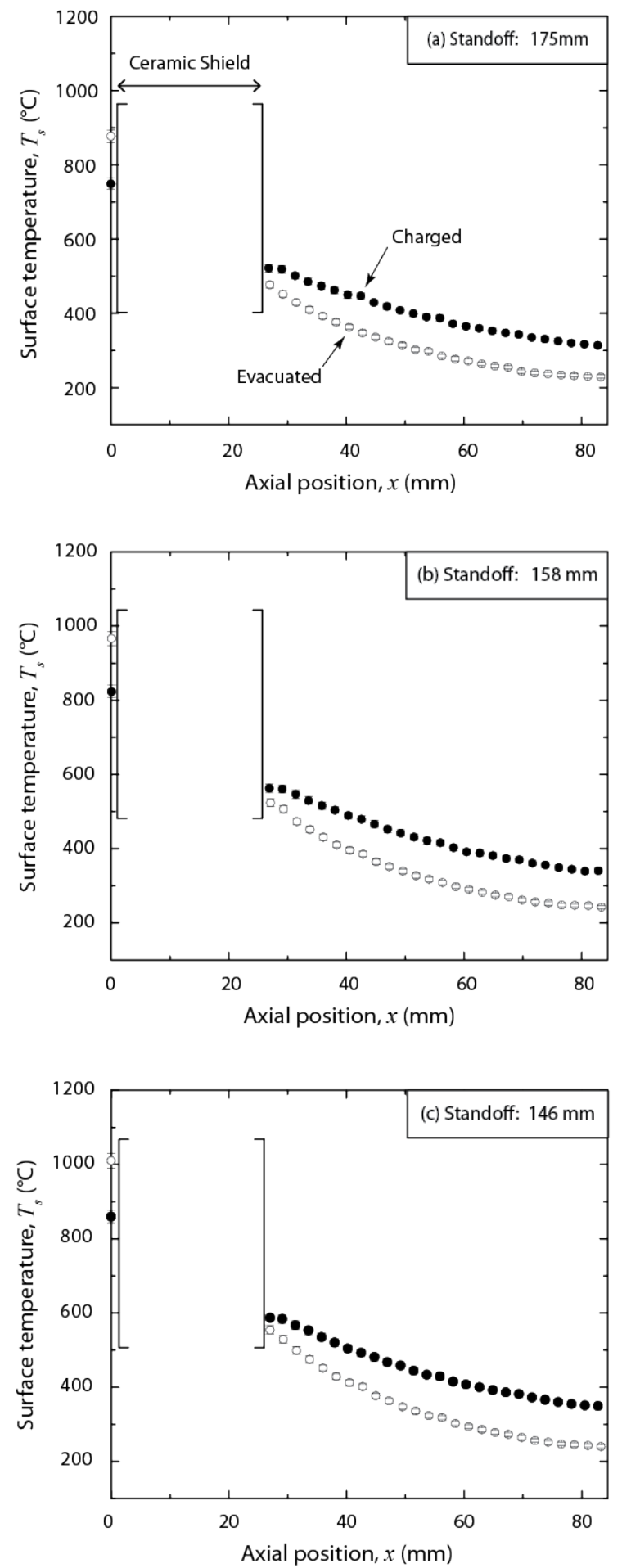

Figure 7.7. Plots comparing the steady state surface temperature profiles of the empty and charged systems at three standoff positions: (a)175mm, (b)158mm, and (c)146mm. 
Figure 7.8 plots the maximum axial temperature difference, taken as the difference in the tip IR reading and the rearmost IR reading, as a function of standoff distance. At a standoff distance of $146 \mathrm{~mm}$, significant deterioration at the tip of the evacuated sample was observed due to high temperature oxidation [134]. As a result, smaller standoffs were not attempted. In contrast, the charged sample reached a standoff distance of $97 \mathrm{~mm}$ before any deterioration was observed. The charged sample averaged a temperature difference which was $33 \%$ lower than that of the evacuated sample, consistent with a higher effective thermal conductivity in the axial direction.

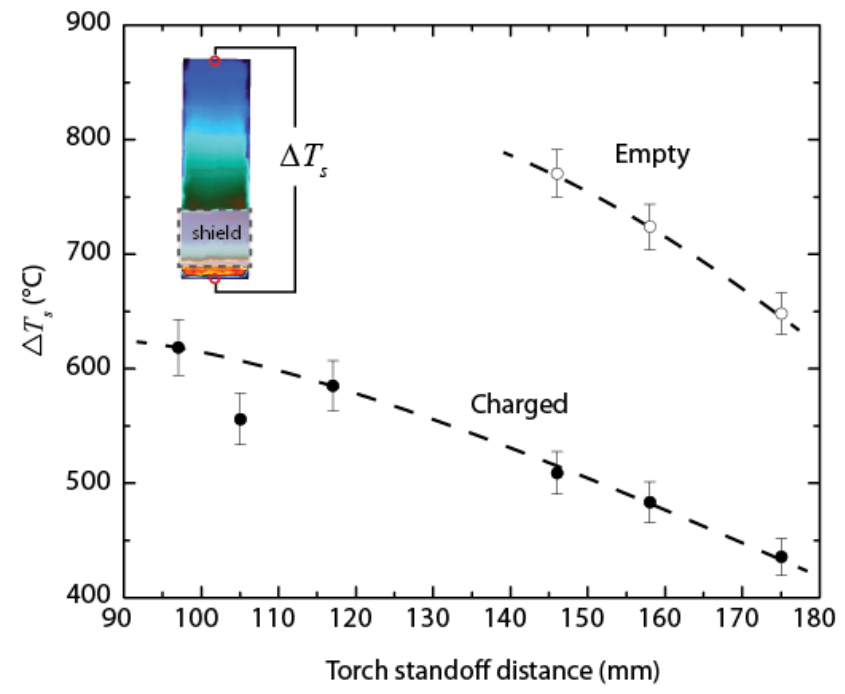

Figure 7.8. Maximum $\Delta T_{\text {s }}$ taken over the sample's length as shown in the inset. The dashed lines are a best-fit approximation.

Despite its lower axial temperature difference, the charged system's external surface was not isothermal. A finite element model was developed to explore this. It incorporated 
the relatively high thermal resistance of the case material (with respect to the effective conductivity of the vapor core).

\section{4. $\quad$ Finite element model}

The quarter symmetry F.E. model presented in Chapter 6 was adapted to the leading edge geometry and experimental boundary conditions for the IN718/Na system used here. The finite element model was solved using SolidWorks ${ }^{\circ}$ Simulation running an iterative FFE solver algorithm. Figure 7.1 shows the mesh used in the study. During trial solutions, a finer mesh than that shown was examined around the curved tip region where the input heat flux was greatest, but the difference in converged solutions was within $3 \%$ so the coarser mesh shown in the figure was used because solution times were $50 \%$ shorter. Convergence criteria was specified to be within $0.1 \%$. As before, the vapor core is modeled as a high thermal conductivity solid with $k_{\text {eff }}=3,000 \mathrm{~W} / \mathrm{mK}$. A rule-of-mixtures estimate of the wick's saturated thermal conductivity, $k_{w i c k}$, was used;

$$
k_{w i c k}=k_{L} \varepsilon+(1-\varepsilon) k_{S}
$$

where $k_{L}$ is the thermal conductivity of the liquid sodium, $k_{S}$ is the thermal conductivity of the nickel cell walls, and $\varepsilon$ is its porosity. The temperature dependent thermal conductivity of the fluid and nickel are given in Appendix A. The model geometry is identical to the asfabricated samples except that the fill tube and rear face plate were not included. 
(a)

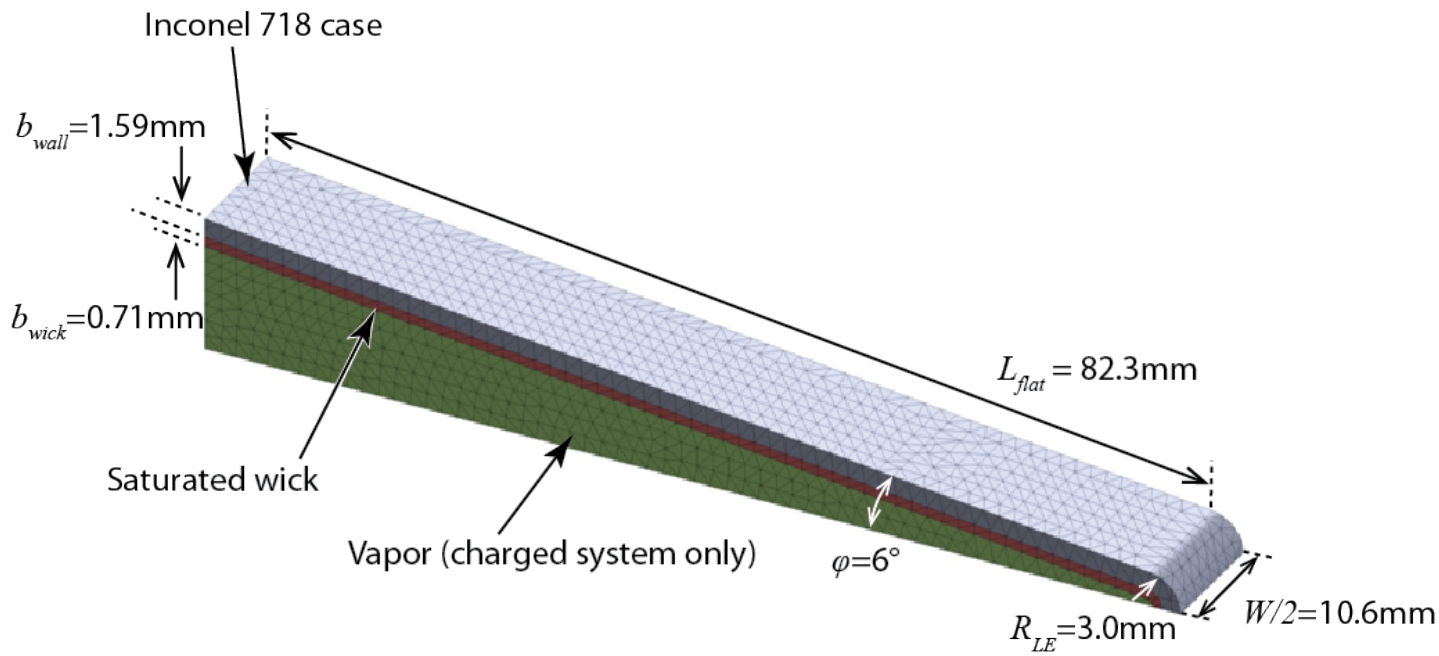

(b)

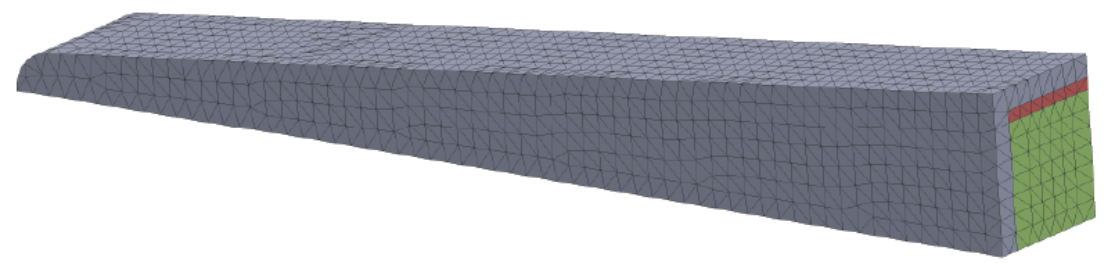

Figure 7.1. Two views [(a) and (b)] of the finite element mesh superimposed on the solid model.

\section{4(a) Boundary Conditions}

The boundary conditions applied to the F.E. model are prescribed by Figure 7.2. The heat flux imposed over the curved tip is prescribed by $q_{\text {wall }}$ (which was kept uniform over the curved portion). The portion of the upper surface which is blanketed by the 
ceramic shield is treated as adiabatic while the exposed region is cooled by a combination of natural convection to the ambient (at temperature $T_{\infty}=22^{\circ} \mathrm{C}$ and with heat transfer coefficient $h_{\text {cool }}=12 \mathrm{~W} / \mathrm{m}^{2} \mathrm{~K}$ ) and surface radiation (with surface emissivity of $\varepsilon=0.95$ ). All other surfaces are treated adiabatic and all interface contact points (wall/wick/vapor) were given a thermal resistance of zero.

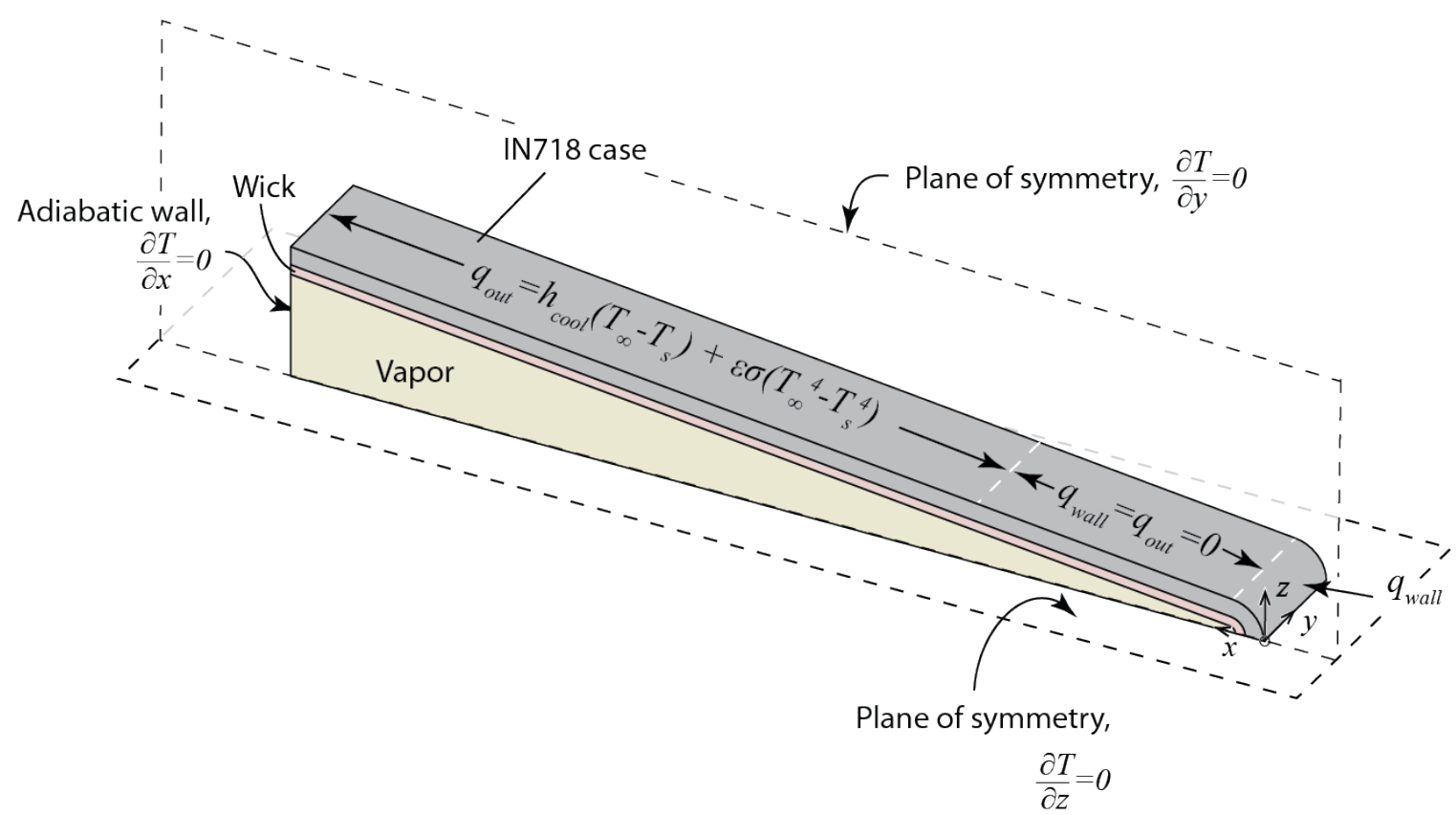

Figure 7.2. Isometric view of quarter-symmetry charged model showing the applied boundary conditions. The evacuated model is identical but has no vapor core and its wick interior surface is treated adiabatic.

\section{4(b) Solution}

We turn now to the challenge of determining $q_{\text {wall }}$, the heat flux absorbed by the wall at the tip and ultimately, at steady state, the power transferred through the wedge structure. For the low-temperature experiments presented in Chapter 7, a thermal resistance 
was determined from the evacuated system model using the measured heater temperature as input. Because a thermal resistance characterizes heat input irrespective of test sample (it is a property of the boundary condition only), it could be used to ascertain the heat absorbed by the charged system as well. Difficulties in measuring the flame temperature and accurately representing its distribution in the F.E. model makes a similar approach for the high temperature case much more problematic. Therefore, a different approach was taken here.

We started by determining the flux absorbed for the simpler evacuated sample. The model of the evacuated system was solved with an initial guess for $q_{\text {wall }}$, and the resulting temperature prediction was compared to the steady state experimental temperature data. This process was iterated changing $q_{\text {wall }}$ until good agreement between the model and data were obtained as shown in Figure 7.9 (I). Because $q_{\text {wall }}$ is dependent on the absorbing system's thermal response, simply applying the obtained values to the charged system in the same way a thermal resistance can be applied is incorrect. 
I. Evacuated (no working fluid)
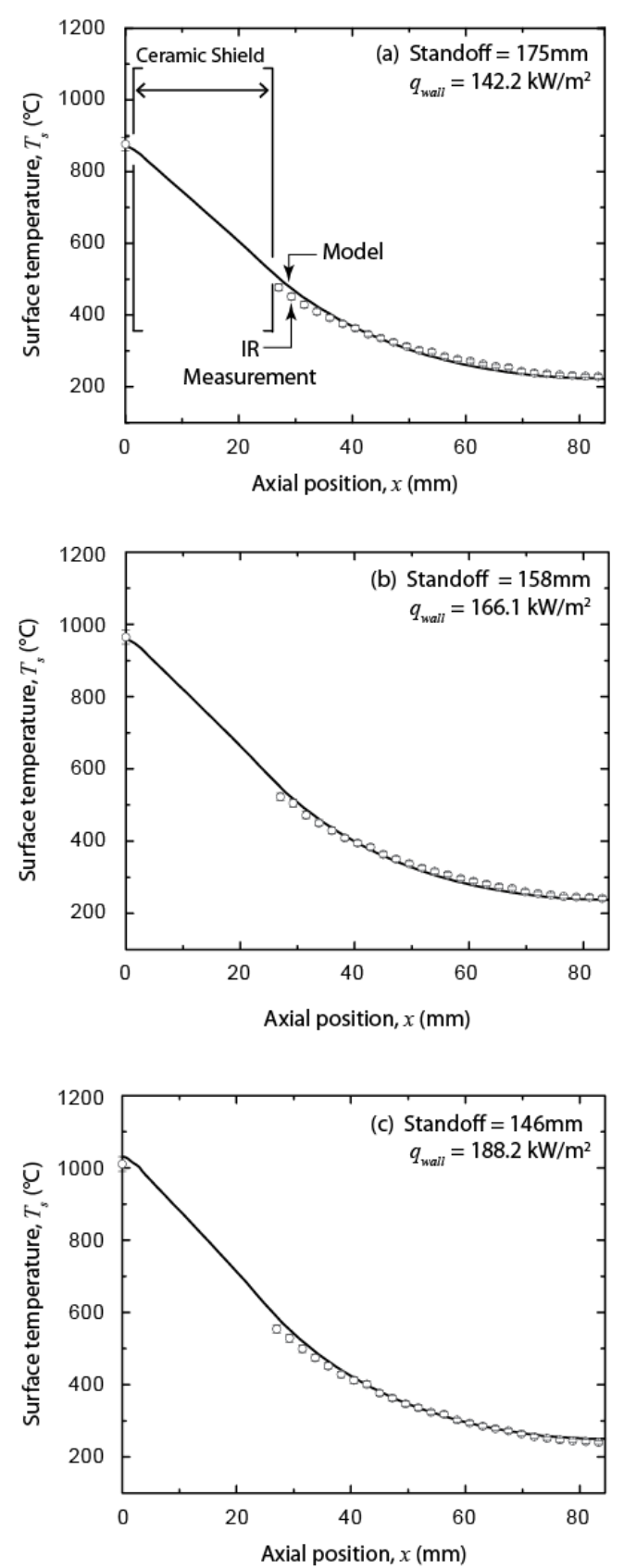

II. Charged (working fluid)
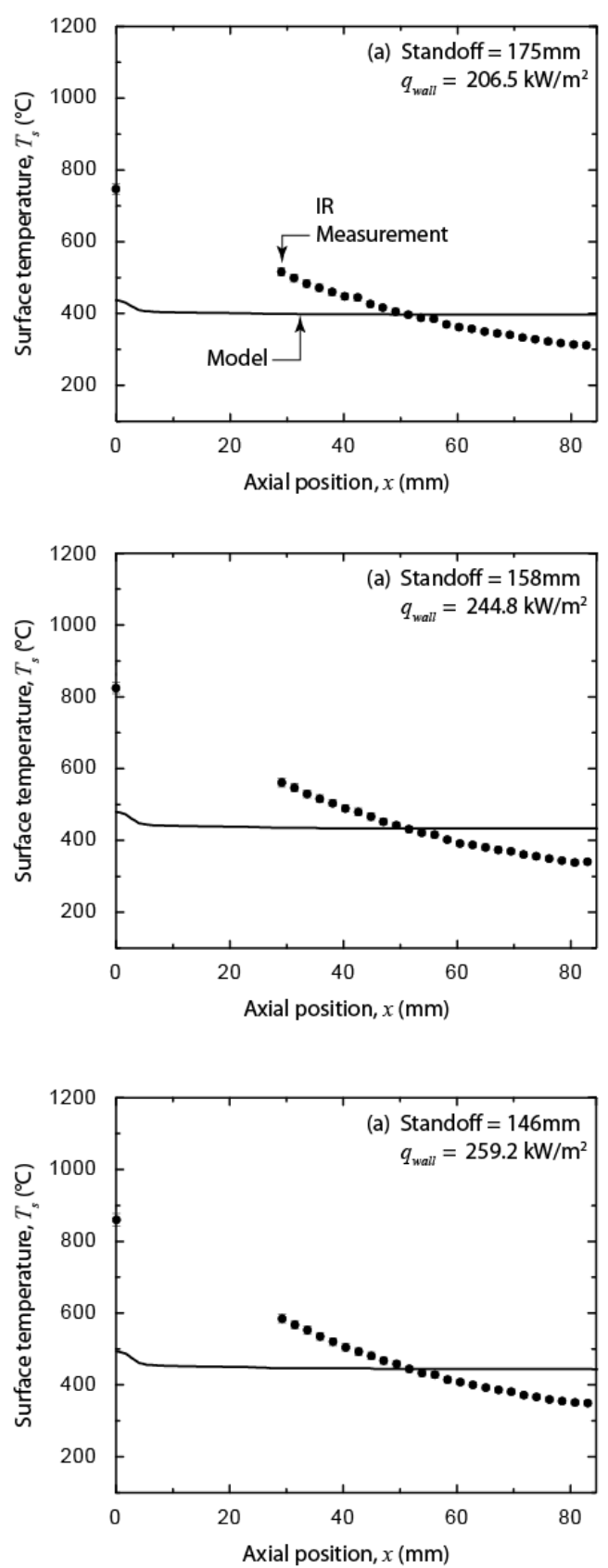

Figure 7.9. Steady state thermal profile plots of (I) the iterative FE solution and experiment for the evacuated sample which confirmed the boundary conditions of the model, and (II) a comparison of the simulation and experiment for the charged sample. 
Instead, we calculate the total power absorbed, $Q_{\text {wall }}$, from the product of $q_{\text {wall }}$ and the surface area of the curved region, $A_{c}$;

$$
Q_{w a l l}=q_{w a l l} A_{c}
$$

At steady state, this value should balance the total power emitted from the sample's surface, $Q_{\text {out }}$, which can be determined from the IR surface measurements on the flat portion of the wedge using;

$$
Q_{\text {out }}=W \sum_{i=1}^{n} \delta x\left[h_{\text {cool }}\left(T_{\infty}-T_{s, i}\right)+\epsilon \sigma\left(T_{\infty}^{4}-T_{s, i}^{4}\right)\right]
$$

where $\delta x$ and $n$ are the distance between temperature readings and the total number of temperature readings, respectively. Values of $Q_{\text {wall }}$ were found to be at most $12 \%$ greater than the experimentally determined $Q_{\text {out }}$ for the evacuated system. The discrepency is likely the result of not being able to experimentally replicate the perfectly insulated model. However, the values are close enough that they lend confidence to the method of estimating the power transferred by the charged samples via equation (7.2). It should also be noted that the measured tip temperatures compare favorably to the model predictions for the evacuated sample (Figure 7.9(I)), suggesting that interference from the torch gases on the IR measurements in this region are reasonably small.

Figure 7.9 (II) compares the experimental results of the charged sample to the simulation. The model's $q_{\text {wall }}$ boundary condition, determined using the above method, is 
labelled in the figure. There exists poor agreement between the simulation and experimental observations; the sample was operating far from the isothermal prediction.

\subsection{Results and discussion}

Figure 7.10 shows the measured steady state axial temperature profiles of the previous figure along with four additional test runs at shorter standoff distances (higher $\left.q_{\text {wall }}\right)$. The test at $q_{\text {wall }}=434.3 \mathrm{~kW} / \mathrm{m}^{2}$ corresponds to the emergence of an inflection point in the temperature profile which persists at the high flux levels. The profile shape is not consistent with solid-state conduction; mass transfer processes are active.

As the heat flux is increased to over $434.3 \mathrm{~kW} / \mathrm{m}^{2}$, the condenser temperature begins to isothermalize as the inflection point shifts down the length of the test sample. Unlike lower flux levels, there is very little change in tip temperature during the initial stages of this progression.. There is, however a nearly $100^{\circ} \mathrm{C}$ jump in tip temperature as the heat is increased from $q_{\text {wall }}=563.8$ to $671.2 \mathrm{~kW} / \mathrm{m}^{2}$. 


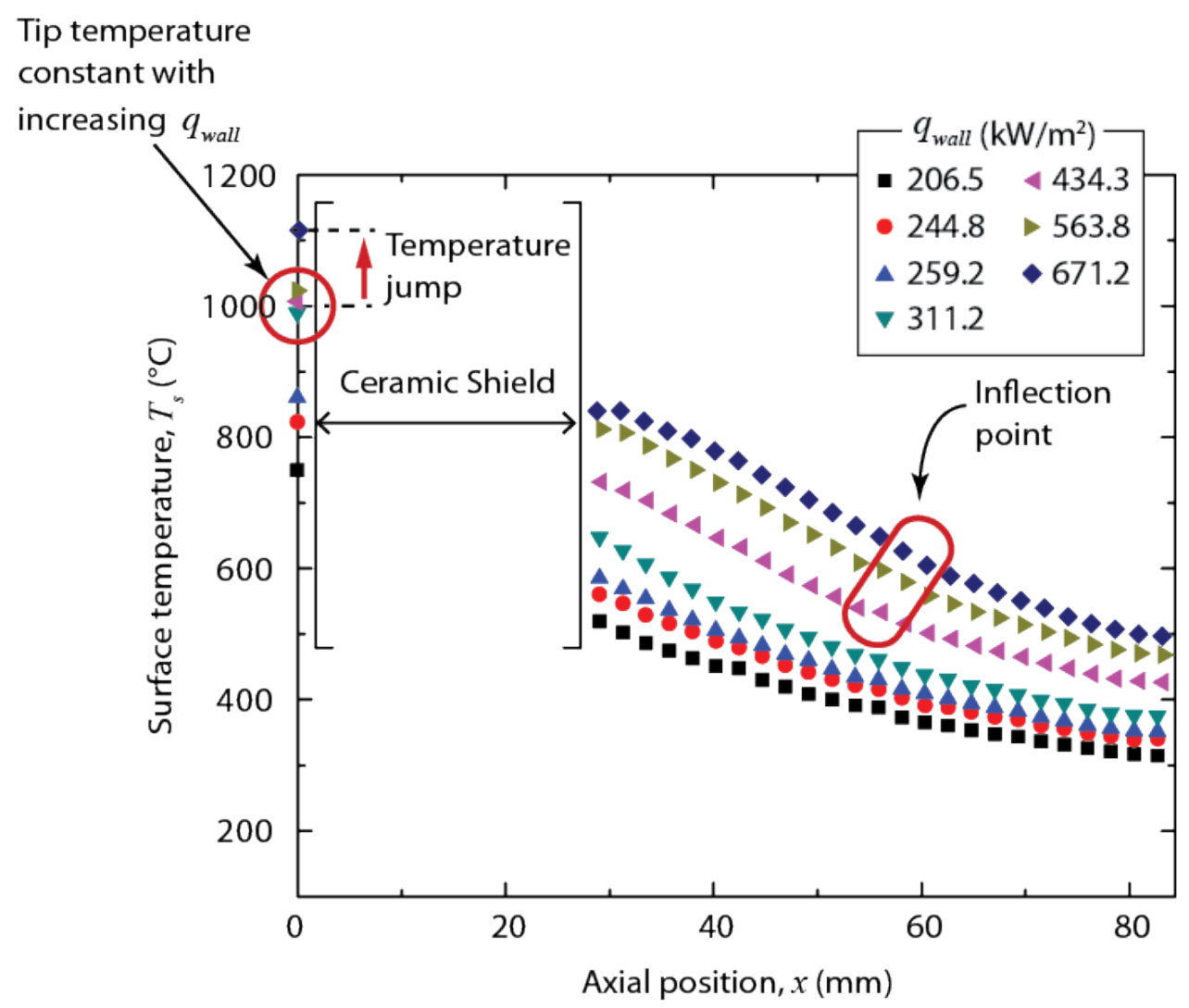

Figure 7.10. Axial temperature profiles of the charged system for seven standoff positions. There is the emergence of an inflection point at position $434.3 \mathrm{~kW} / \mathrm{m}^{2}$, which persists through tests at $563.8 \mathrm{~kW} / \mathrm{m}^{2}$ and $671.2 \mathrm{~kW} / \mathrm{m}^{2}$. Error is $\pm 2 \%$ of the temperature reading; errors bars have been removed for clarity.

The F.E. model assumes the vapor always acts as a highly effective thermal conductor. Should the sample's operation be encumbered by vapor transport limits, this would lead to the poor agreement between experiment and model.

All previous discussion in this work had assumed a vapor flow in the continuum state. Cao and Faghri [135] noted that at very low vapor pressures, a flow condition may exist in which vapor transport down the heat pipe occurs under molecular self-diffusion 
rather than pressure-driven convection. Models have shown this self-diffusion mechanism to be slow, resulting in large thermal gradients and operation which is far from isothermal [135]. The transition from molecular flow to continuum flow is characterized by the Knudsen number, which is the ratio of the mean free molecular path, $\lambda_{m}$, to a characteristic length of the system, $D$;

$$
\mathrm{Kn} \equiv \lambda_{m} / D
$$

It is generally considered that the transition from molecular/slip flow to continuum flow occurs at $\mathrm{Kn}=0.01$ [136]. At values higher than this, the mean free path is comparable to the length scale of the container where vapor transport operates under diffusive phenomena. Using the kinetic theory of gases, the perfect gas law, and assuming the liquid and vapor are in saturation to make use of the Clausius-Clapeyron equation, an expression containing the transition or Knudsen temperature, $T_{t r}$, between rarefied and continuum flow can be found [135];

$$
\ln \left(\frac{1.051 \kappa T_{t r}}{\sqrt{2} \pi \sigma_{d}^{2} \mathrm{Kn} P_{V, R E F} D}\right)+\frac{\lambda M}{R}\left(\frac{1}{T_{t r}}-\frac{1}{T_{V, R E F}}\right)=0
$$

where $\kappa$ is the Boltzmann constant $\left(=1.38 \times 10^{-23} \mathrm{~J} / \mathrm{K}\right)$ and $\sigma_{d}$ is the collision diameter $\left(=3.72 \times 10^{-10} \mathrm{~m}\right.$ for sodium [137]). Expression (7.5) requires an iterative solution for $T_{t r}$. Approximating $D \approx 2 R_{L E}$, taking $\mathrm{Kn}$ as 0.01 , and with $\lambda=4260 \mathrm{~kJ} / \mathrm{kg}$, it follows that $T_{t r}=532^{\circ} \mathrm{C}$. This seems to agree well with the experiemental results, which shows the formation of the inflection point (marking the start of significant vapor transport) when the 
condenser surface temperature (and vapor temperature) is in the 500 to $600^{\circ} \mathrm{C}$ range. The coupling of $T_{t r}$ to $D$ is weak; a doubling of $D$ gives $T_{t r}=493^{\circ} \mathrm{C}$ which still agrees reasonably well with what was observed.

The experimental data suggests the possibility that the heat spreader's operation transitions from a rarefied gas vapor core, where transport of the latent heat is dominated by self-diffusion of the vapor molecules, to one of continuum flow where convection more efficiently spreads the heat down the length of the sample. Figure 7.11 illustrates the proposed internal operation and corresponding temperature profile for this situation. At low applied heat fluxes $\left(q_{1}\right)$, the vapor temperature is below the Knudsen transition temperature. Here the vapor is rarefied so that the net diffusion of molecules down the vessel governs heat transport and is driven by a small density gradient arising from the vapor's axial temperature difference. Heat transfer is primarily driven by thermal conduction along the wall. An increase in heat flux $\left(q_{2}\right)$ leads to an increase in temperature, particularly at the tip whose vapor can locally rise above the Knudsen temperature. The commensurate increase in pressure (from a rise in evaporation rate) decreases the mean free path of molecules leading to a local transition to continuum flow. The latent heat is convectively transported through the vapor core until the molecules encounter the rarefied gas front which persists in the region where the vapor temperature remains below the Knudsen temperature. To balance the efficient transfer of mass by convection with that of diffusion at the continuum-rarefied gas interface, there is a high rate of vapor condensation in the continuum flow regime. The surface thermal profile reflects this abrupt change in heat transfer mechanisms with a rapid 
drop in temperature at the gas interface. With a further increase to the impinging heat flux $\left(q_{3}\right)$, the continuum flow region grows downstream. If the heat flux increase is large enough, the capillary limit may be reached leading to a dryout condition in the wick and a temperature spike. These trends are similar to what was observed, shown in Figure 7.10, where analogously $q_{1}=206.5$ to $311.2 \mathrm{~kW} / \mathrm{m}^{2}, \quad q_{2}=434.3$ to $563.8 \mathrm{~kW} / \mathrm{m}^{2}$, and $q_{3}=671.2 \mathrm{~kW} / \mathrm{m}^{2}$.

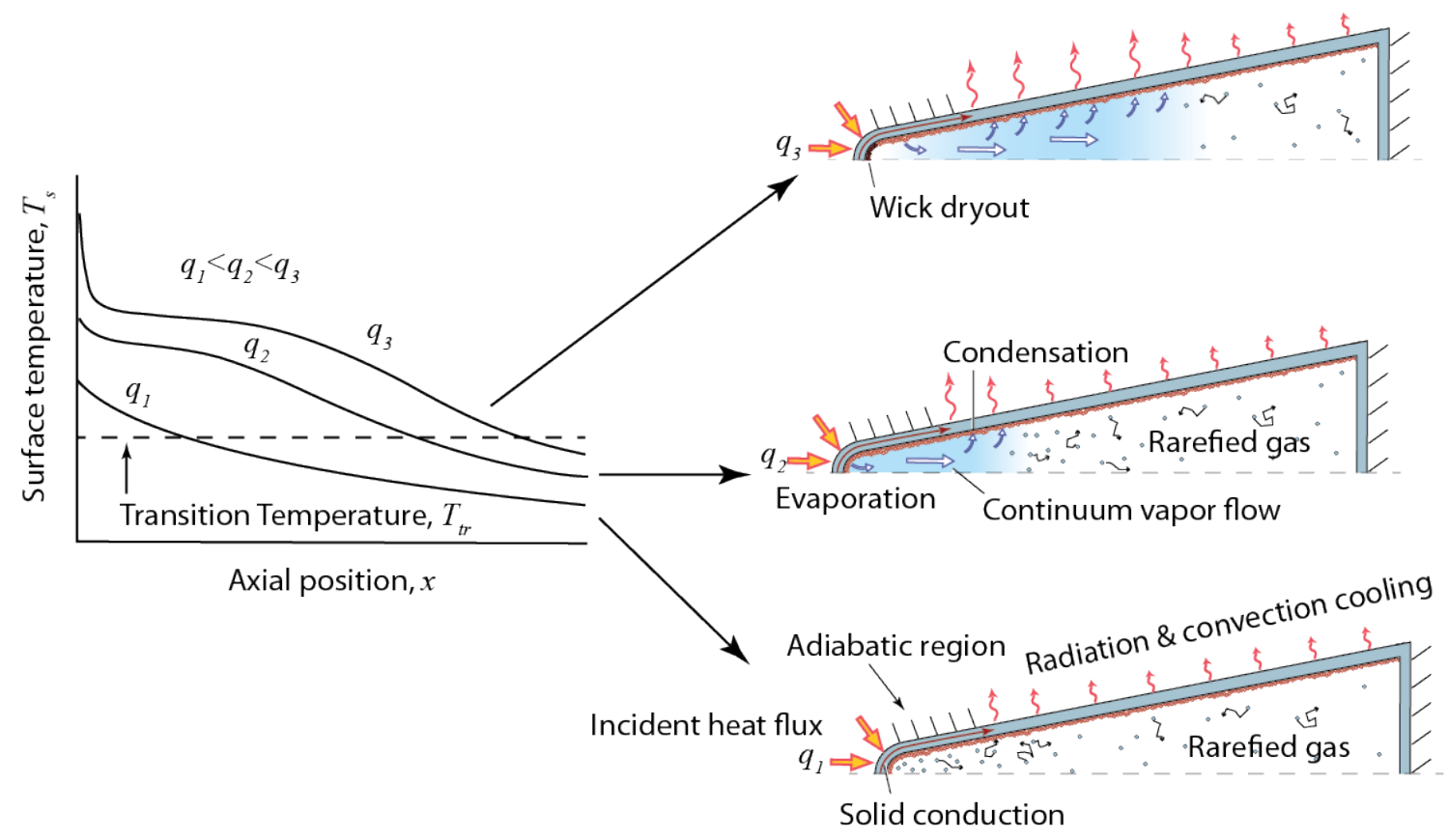

Figure 7.11. Illustration showing the vapor core transition from a rarefied gas, to a continuum vapor, to a dryout condition and each operating regime's representative temperature profile.

In Chapter 4 we found good agreement on the capillary limit at $F=0.05$ for the cylindrical case. Referring to Figure 7.12, we were operating above this level (434.3 and $563.8 \mathrm{~kW} / \mathrm{m}^{2}$ ) with no apparent dryout. The dryout event occurred at $q_{\text {wall }}=671.2$ 
$\mathrm{kW} / \mathrm{m}^{2}$, close to $F=0.4$. The apparent discrepancy is further evidence of the important role which thin film evaporation plays in the capillary limitation of heat pipes and demonstrates the need for its special consideration in modeling of the limit.

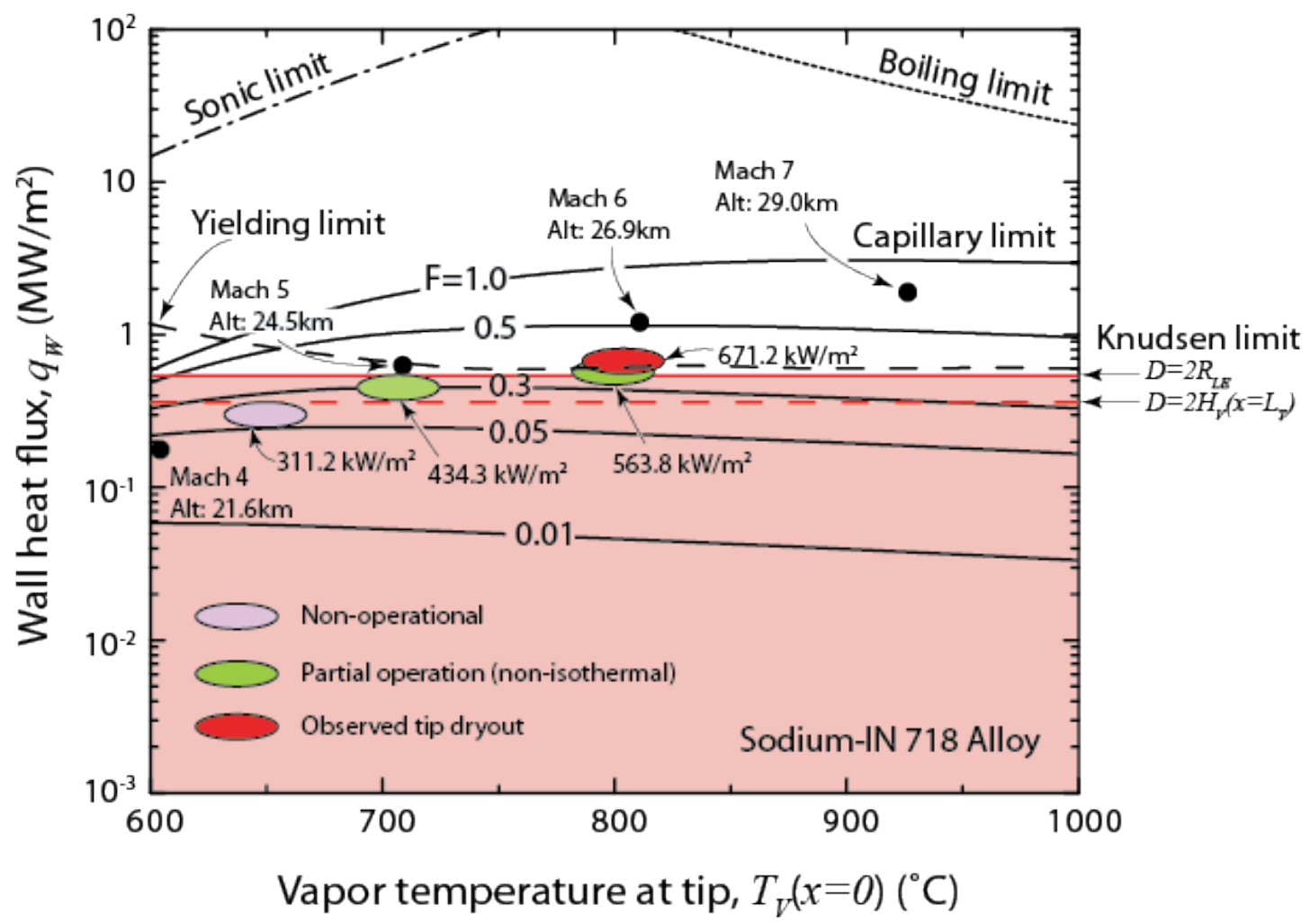

Figure 7.12. Sodium-IN718 limits map showing the regions probed by the experimental work. The vapor temperature is estimated to be equivalent to the surface temperature immediately aft of the ceramic shield. The shaded area indicates a region made non-operational by the Knudsen limit (for $D=2 H_{V}(x=0)$ ).

Because the Knudsen number distinguishes the transition between molecular and continuum flow, and therefore the transition between non-isothermal and isothermal operation, it would be valuable to pose it in the form of an operating limit which can be 
plotted on the limits map, particularly for when low vapor pressure working fluids such as liquid metals are used.

Referring back to equation (7.5), a transition temperature $T_{t r}$ was solved which delineated the minimum condenser vapor temperature for isothermal operation. Therefore, using a heat flow balance between the leading edge tip and condenser, the minimum flux at the tip for isothermal condenser operation must be;

$$
q_{K n}=\frac{L_{\text {flat }}}{\left(\frac{\pi}{2}-\varphi\right) R_{L E}}\left(h_{\text {cool }}\left[T_{\infty}-T_{t r}\right]+\epsilon \sigma\left[T_{\infty}^{4}-T_{t r}^{4}\right]\right)
$$

where the first term on the right hand side quantifies convection cooling and the second describes radiation cooling. Equation (7.6) is an expression for a lower operating limit, referred to here as the Knudsen limit, which is dependent only on the transition temperature - a property of the working fluid - the leading edge tip curvature and length, and the thermal boundary conditions. It is plotted horizontally in Figure 7.12 (for $D=2 H_{V}(x=$ $0)$ and $2 H_{V}\left(x=L_{e}\right)$, their minimum and maximum values) to reflect that it is independent of vapor temperature in the evaporator. Only at fluxes above this limit is the leading edge able to operate isothermally (subject to the sonic, capillary, and boiling limits). 


\section{Chapter 8. Discussion}

In this chapter, the design model (developed in Chapter 3) and heat transport limit model (developed in Chapters 4 and 5) are applied together to formulate a cohesive methodology for the design of metallic, wedge-shaped leading edge heat pipes. The methodology is framed within the context of the low and high temperature experimental systems. Afterwards, the potential of wedge-shaped heat spreaders for a hypersonic vehicle TPS is considered.

\subsection{Two-model design methodology}

Two distinct models make up the design methodology:

(a) Design model. Hypersonic flow theory was used to generate a design maps which succinctly present the relationship between geometric parameters (condenser design length, wall thickness) and operating conditions (wall temperature, tip temperature, and thermally- 
induced tip stress) given a case alloy, working fluid, and flight parameters (Mach number and altitude); and,

(b) Limits model. Vapor transport theory was used to create a limits map which identifies the maximum heat flux that can be transferred through the leading edge tip as a function of the vapor stagnation temperature. The limit map is dependent on geometric parameters and material selection (case alloy and working fluid).

The design map delineates a boundary for workable designs so long as the heat transport limits are not encountered, i.e. its validity is predicated on operation below the transport limits. Therefore, a comprehensive design approach which incorporates both the design theory and limit theory is necessary. With the leading edge radius and wedge half angle typically set by aerodynamic requirements, then, as a first step, the design map should be generated (for a given Mach number and altitude) from which a wall thickness and design length can be chosen subject to any isothermal operating temperature, maximum (tip) temperature, or tip stress requirements. The wall thickness and design length are then used, along with the choice of wick design, to create the limit map. If the vehicle's flight parameter (Mach number, altitude) generates a wall heat flux falling outside the safe operating regime on the limits map, a change to one or more of the design parameters is necessary. Table 8.1 summarizes the influence of the geometric parameters and material selection on the heat flux limits. 
Table 8.1. Influence of design parameters and material selection on limits for a leading edge with radius $R_{L E}$ and half angle $\varphi$.

\section{Parameter To increase limit:}

\section{Capillary Limit}

Wall thickness, $b_{\text {wall }}$

Wall thermal conductivity, $k_{\text {wall }}$

Wick thickness, $b_{\text {wick }}$

Wick thermal conductivity, $k_{\text {wick }}$

Wick pore radius, $R_{P}$

Wick permeability, $K$

Design length, $L_{\text {flat }}$

\section{Yielding Limit}

Wall thermal conductivity, $k_{\text {wall }}$

Wall material's Young's modulus, $E$

Wall coefficient of thermal expansion, $\alpha$

Wall thickness, $b_{\text {wall }}$

Wall yield strength, $\sigma_{y}$

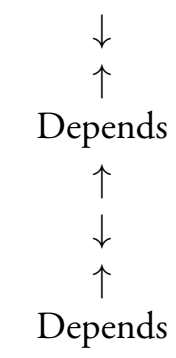

\section{Sonic Limit}

Depends on working fluid selection only.

\section{Boiling Limit}

Wick thermal conductivity, $k_{\text {wick }}$

Wick thickness, $b_{\text {wick }}$

Wall thickness, $b_{\text {wall }}$

To decrease limit:

\section{Knudsen Limit}

Design length, $L_{\text {flat }}$ 


\section{$\underline{\text { Low temperature system }}$}

Figure 8.1 shows the design map for the SS304-water system whose experimental investigation was described in Chapter 6. The design point for a system with $L_{\text {flat }}=$ $160.3 \mathrm{~mm}$ and wall thickness is $b_{\text {wall }}=1.59 \mathrm{~mm}$ is shown. The wall heat flux used to create the map was captured from the F.E. simulations. The map predicts a maximum (tip) temperature of $\approx 89^{\circ} \mathrm{C}$ and an isothermal vapor temperature, found by following its $b_{\text {wall }}$ line contour to the abscissa, of $\approx 67^{\circ} \mathrm{C}$. During testing, the maximum (tip) temperature was $T_{\text {max }} \approx 80^{\circ} \mathrm{C}$, a difference of $11 \%$ from that predicted by Figure 8.1 . The observed isothermal (surface) temperature was $T_{s} \approx 68^{\circ} \mathrm{C}$, a difference of less than $2 \%$ from the predicted temperature. Predicted stresses are well below the yield strength of SS304.

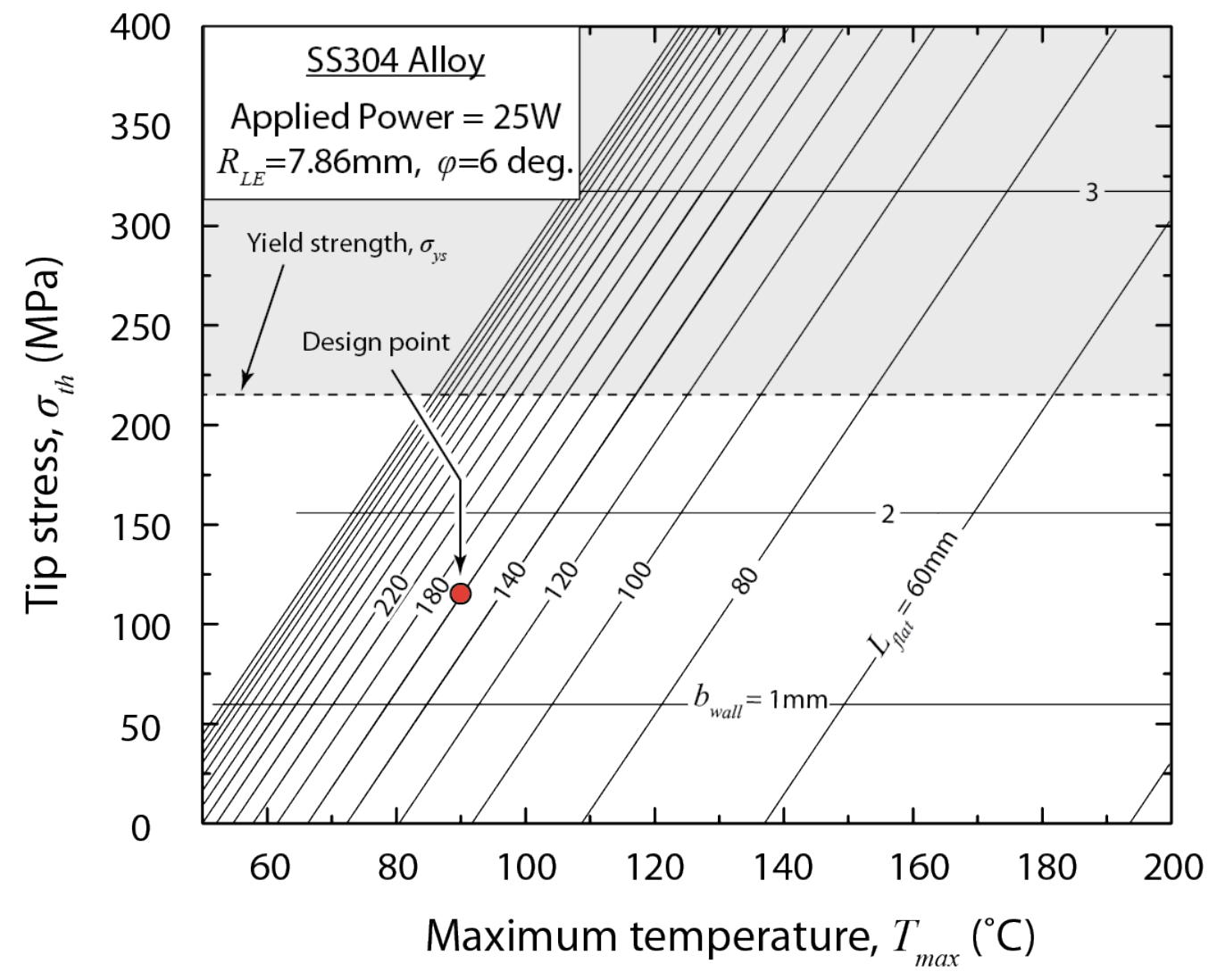


Figure 8.1. Design map for the SS304 wedge subjected to 25W over its tip curvature. The yield strength-temperature curve for SS304 is well above the plot's range.

The design model assumes isothermal operation; therefore, the design map of Figure 8.1 is valid only if the system does not encounter a heat transport limit. We now turn to the limits map of Figure 8.2 to assess the validity of the temperature and stress predictions. The sonic limit (greater than $9 \mathrm{MW} / \mathrm{m}^{2}$ over the temperature range), boiling limit (greater than $47 \mathrm{MW} / \mathrm{m}^{2}$ ), and yielding limit (greater than $800 \mathrm{~kW} / \mathrm{m}^{2}$ ) are well above the probed heat fluxes. At an applied power of $25 \mathrm{~W}$ (wall heat flux of $5.89 \mathrm{~kW} / \mathrm{m}^{2}$ ), the system operates above the predicted capillary limit at $F=0.05$, the fraction approached by the Vinz and Busse [104] heat pipe at its observed limit. While no dryout was observed here, a proper design change to the parameter values outlined in Table 8.1 would increase the capillary limit for more margin in the operating window.

It should be noted that the capillary limit's areal active fraction, $F$, is unknown and likely depends on wick design and liquid superheat. Higher fidelity models and direct experimental observation of evaporating fluids from wicks should be explored to map a correlation between wick structure, heat flux, and other parameters needed attain a reasonable value for $F$ to make use of the modified capillary limit model presented. 


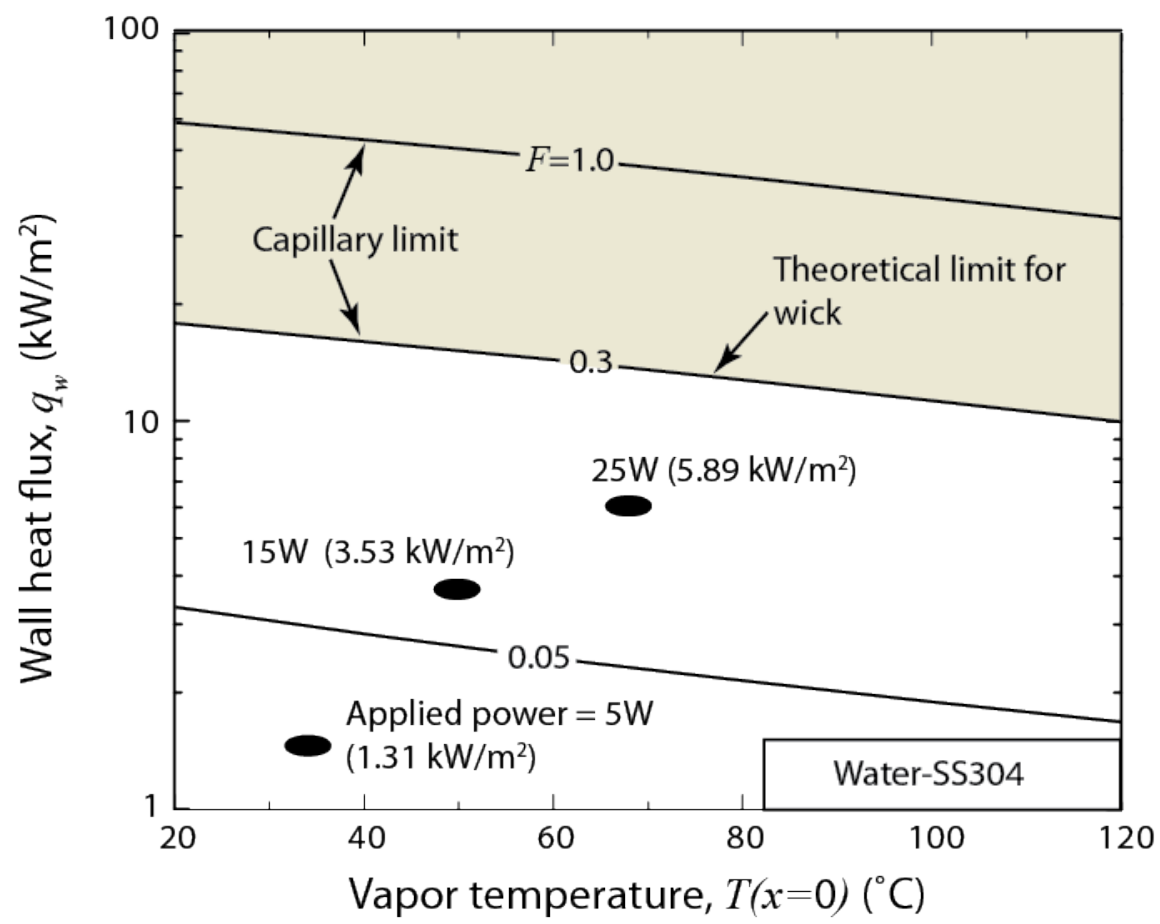

Figure 8.2. Heat transport limits map for the water-SS304 leading edge heat spreader. The sonic, boiling, and yielding limits are not shown because they are well above the plot's range.

\section{High temperature system}

Figure 8.3 shows the Mach $5(24.5 \mathrm{~km})$ design map (duplicated from Chapter 7$)$ for the tested IN718-system. The tested wall heat flux of $q_{\text {wall }}=434.3 \mathrm{~kW} / \mathrm{m}^{2}$ is comparable to a Mach 5 enthalpy condition. At the design point, the anticipated maximum (tip) temperature is $T_{\text {max }} \approx 760^{\circ} \mathrm{C}$ and the isothermal (vapor) temperature is $T_{V} \approx 710^{\circ} \mathrm{C}^{13}$. During testing, the observed maximum temperature was significantly higher $\left(T_{\max } \approx\right.$

${ }^{13}$ The ceramic shield in the experiments effectively shortened the condensing surface $L_{\text {flat }}$ by roughly $1 / 3$. If isothermal operation were observed, the expected temperature would be approximately $20^{\circ} \mathrm{C}$ higher than the design point. 
$\left.1000^{\circ} \mathrm{C}\right)$, and the vapor temperature, approximated by the surface temperature $T_{s}$, ranged from 425 to $725^{\circ} \mathrm{C}$ over the length of the condenser.

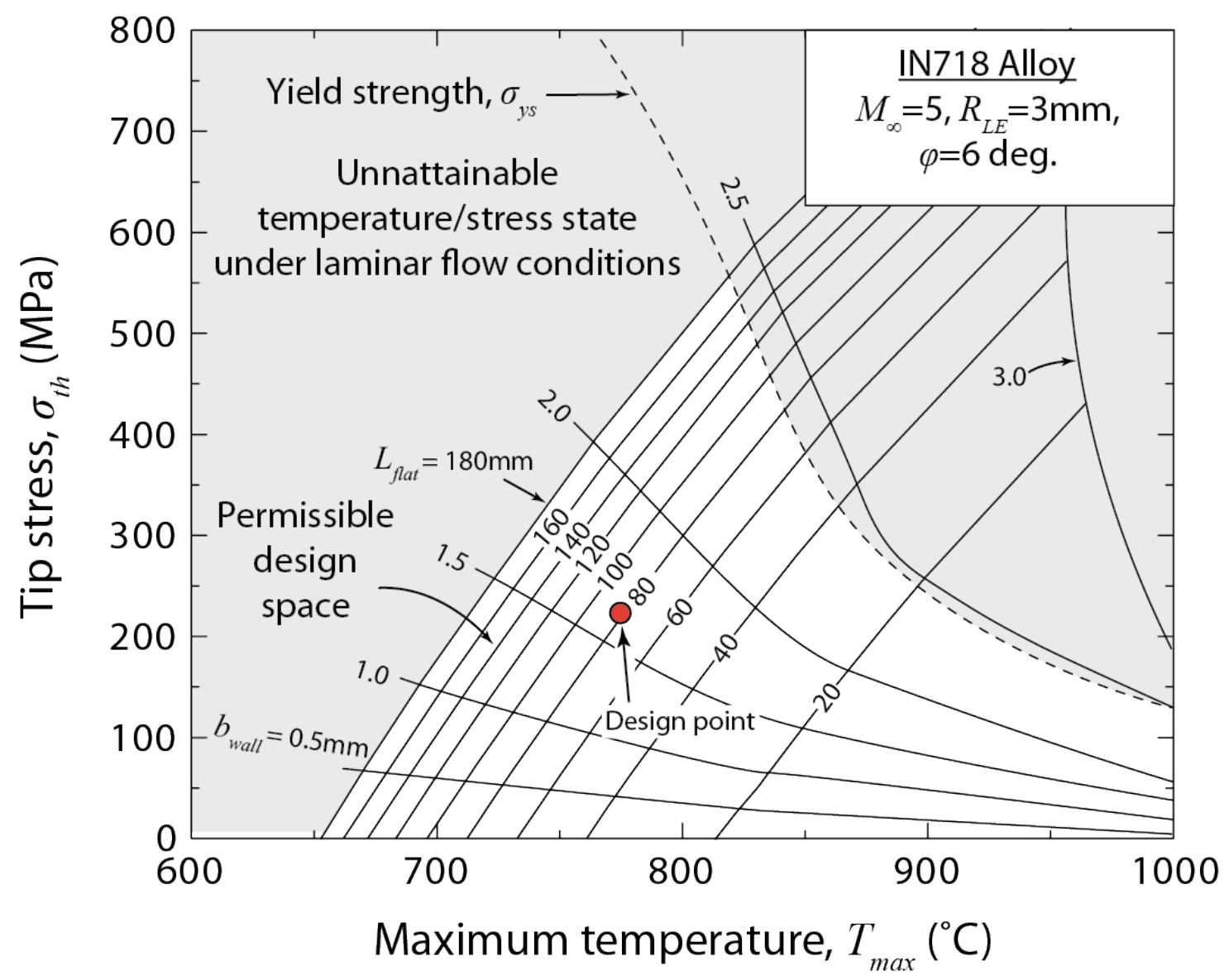

Figure 8.3. Design map for the tested IN718-sodium leading edge under Mach 5 (24.5km) enthalpy conditions.

The limits map (Figure 8.4) suggests a possible cause for non-isothermal operation. Unlike the sonic, boiling, yielding and capillary limits which set the upper bound of the operating regime, the Knudsen limit defines the minimum required wall heat flux for isothermal performance. For the tested leading edge, it is three to four times higher than the 
capillary limit $(F=0.05)$, effectively squeezing the operating regime closed. The limits map predicts that there is no temperature or heat flux combination at which the as-fabricated sodium-IN718 leading edge would operate isothermally.

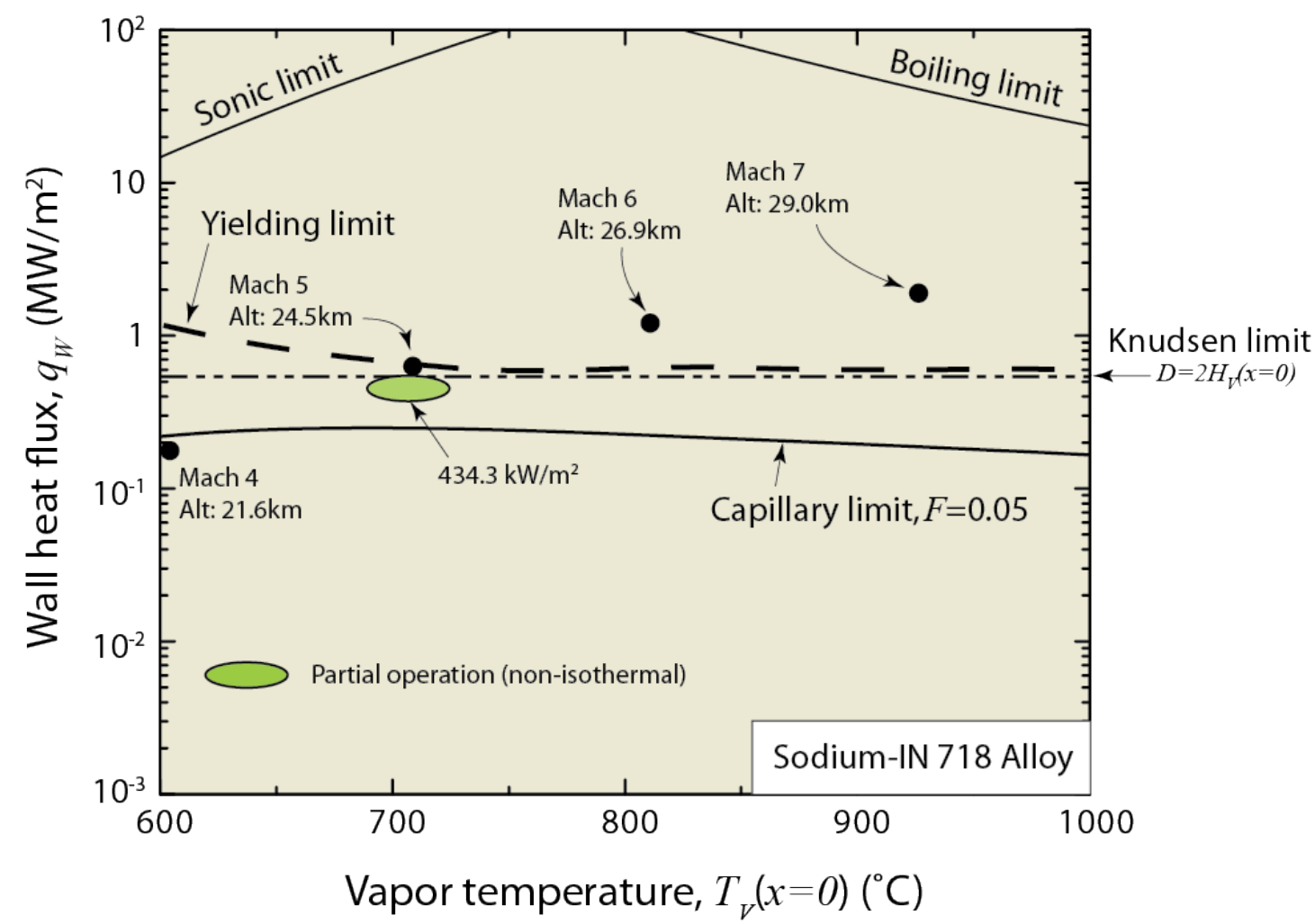

Figure 8.4. Heat transport limits map for the IN718-sodium system with the proposed Knudsen limit. There is no operational window (indicated by a fully shaded plot).

Therefore, design changes are needed to open the operating regime so that a Mach 5 $(24.5 \mathrm{~km}) \mathrm{IN} 718 /$ sodium system can operate isothermally. This can be done through a combination of lowering the Knudsen limit, increasing the capillary limit, and/or lowering or raising the wall heat flux for Mach 5 operation. Each of these are addressed next. 
In essence, leading edges are subjected to an extremely high heat over a very small area (high heat flux). This translates to low cumulative levels of heat in the vapor. Cooling surfaces need only be several times larger than the tip surface. The proportionality $q_{K n} \propto \frac{L_{\text {flat }}}{\left(\frac{\pi}{2}-\varphi\right) R_{L E}}$ in Equation (7.1) quantifies this scaling law: for sharp leading edges the Knudsen limit is high, requiring larger wall heat fluxes than blunt body heat pipes to achieve temperature uniformity. To open up the operating window, $q_{K n}$ must be lowered which can be accomplished by shortening the design length, $L_{\text {flat }}$. Figure 8.5 shows that decreasing $L_{\text {flat }}$ from 82.3 to $41.15 \mathrm{~mm}$ lowers both the Knudsen limit and Mach 5 $(24.5 \mathrm{~km})$ operating heat flux ${ }^{14}$. However, the decrease to $L_{\text {flat }}$ had very little change on the capillary limit (the change is virtually indistinguishable on the plotted axes), which is still below the Mach 5 operating condition.

\footnotetext{
${ }^{14}$ Shorter design lengths increase the wall enthalpy, reducing the driving force for heat transfer in the gas to the tip's wall surface, which leads to the lower wall heat flux..
} 


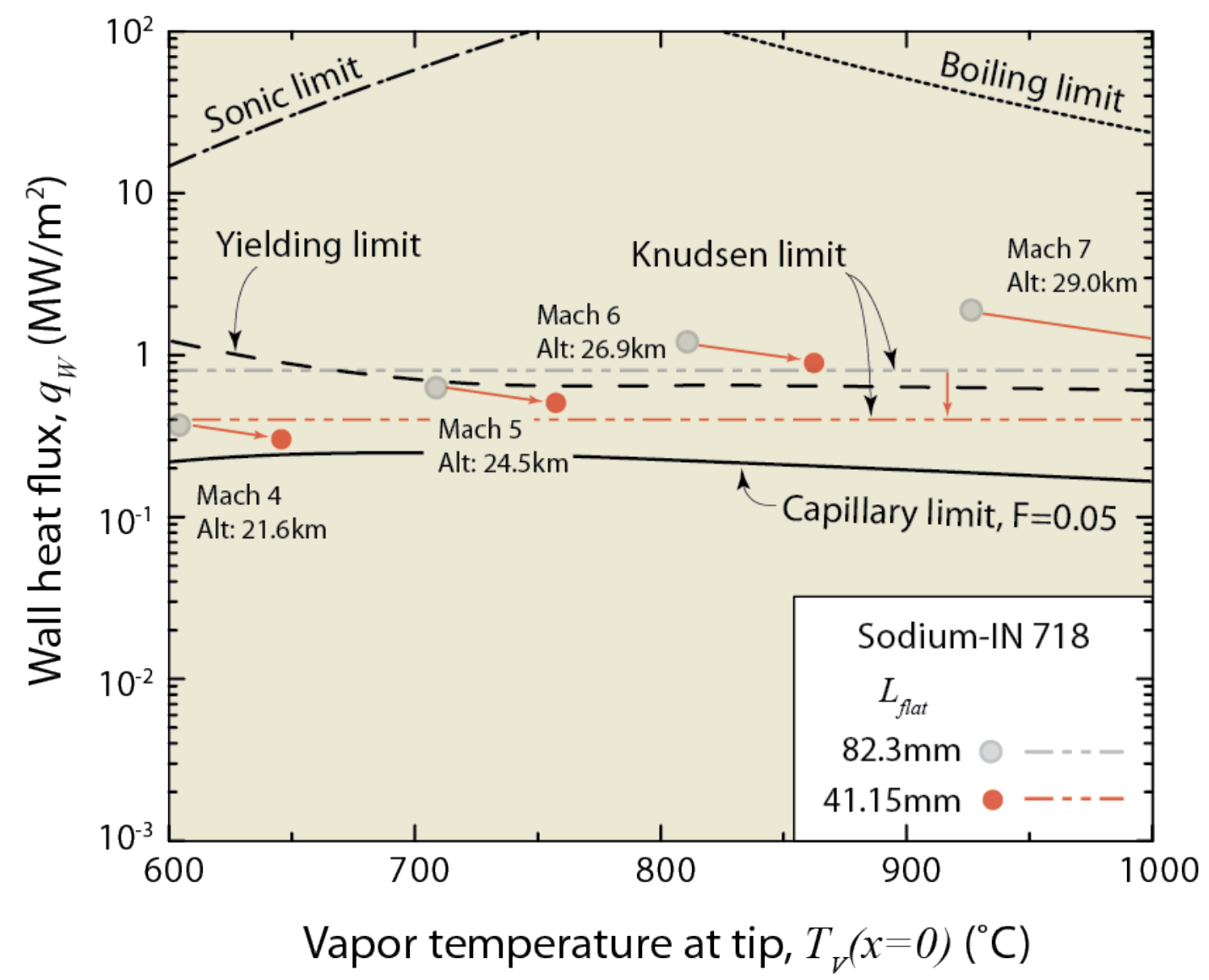

Figure 8.5. Heat transport limits map for the sodium-IN718 system showing the dependence of the Knudsen limit (for $D=2 H_{V}(x=0)$ ) and flight operating condition on the design length, $L_{\text {flat. }}$. There is no operational window (indicated by a fully shaded plot).

One path toward increasing the capillary limit is to decrease the wick's pore radius, $R_{P}$. The effects of this change are shown in Figure 8.6. The operating window opens up for Mach $5(24.5 \mathrm{~km})$ flight when the pore size is decreased to $10 \mu \mathrm{m}$. Note also that the IN718/sodium operating window could be further expanded to serve lower Mach numbers by further decreasing $L_{\text {flat }}$ (which, in turn, lowers the Knudsen limit). However, this comes at the cost of an elevated tip and vapor temperature. 


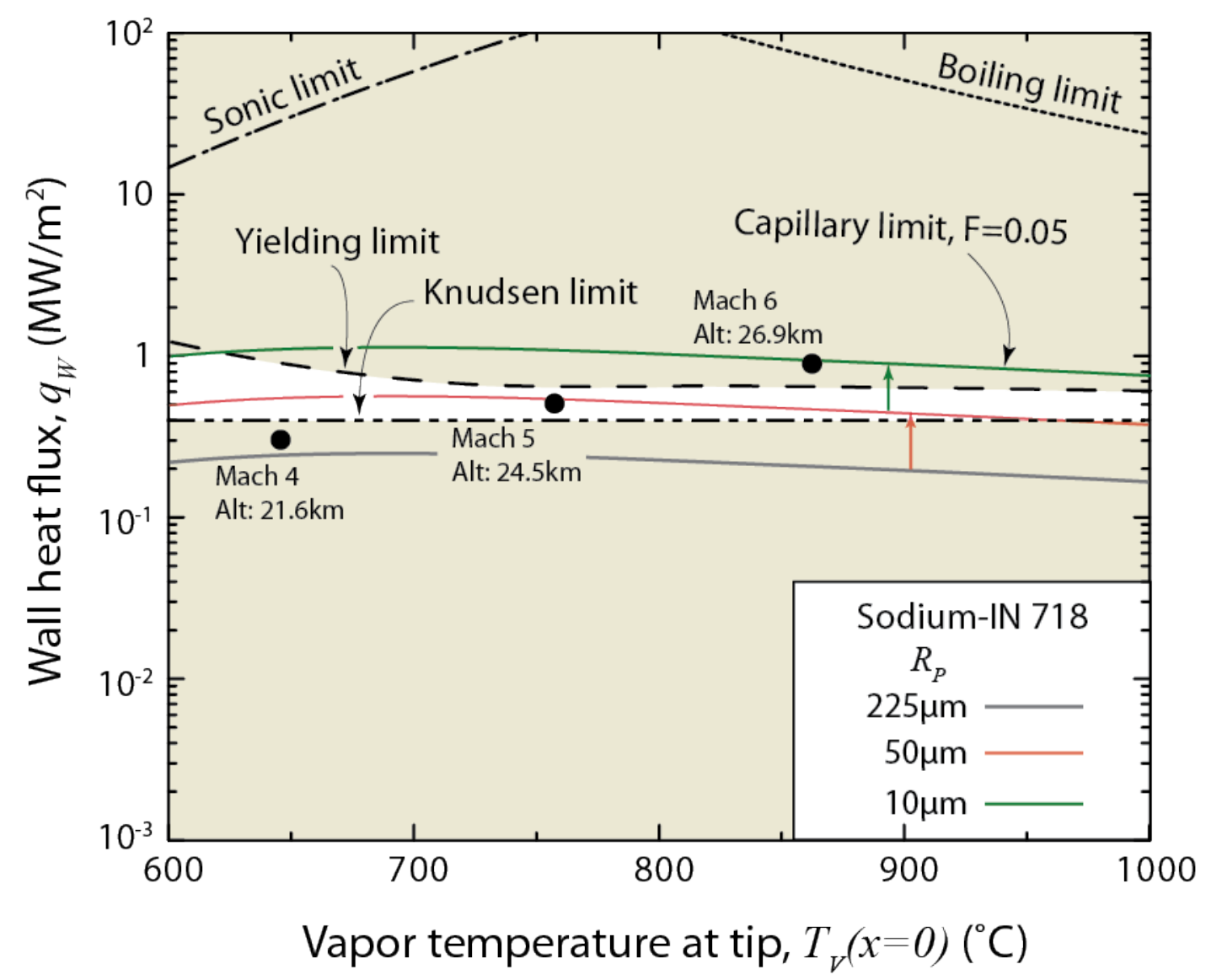

Figure 8.6. Heat transport limits map for an IN718/sodium system showing the dependence of the capillary limit on the wick pore size. The white area delineates the operating window.

\subsection{Assessment of metallic leading edges for hypersonic flight}

In addition to the IN718/sodium heat wedge, the heat transport limit maps below propose a C103/lithium (Figure 8.7) and TZM/lithium (Figure 8.8) leading edge TPS system that would be utilized by air breathing hypersonic vehicles of the future. Their design 
parameters are listed in Table 8.2. A C103/lithium system can be designed to have an operational window from about Mach $5(24.5 \mathrm{~km})$ to Mach $7(29.0 \mathrm{~km})$. It is bounded by the yielding limit over the entire operating window. In contrast, the higher strength of TZM pushes the yielding limit above the capillary limit $(F=0.05)$ to widen the operational window from Mach $7(29.0 \mathrm{~km})$ to above Mach $9(32.3 \mathrm{~km})$. There would be little benefit to increasing the capillary limit since the system is expected to fail by yielding in the tip wall at higher temperatures.

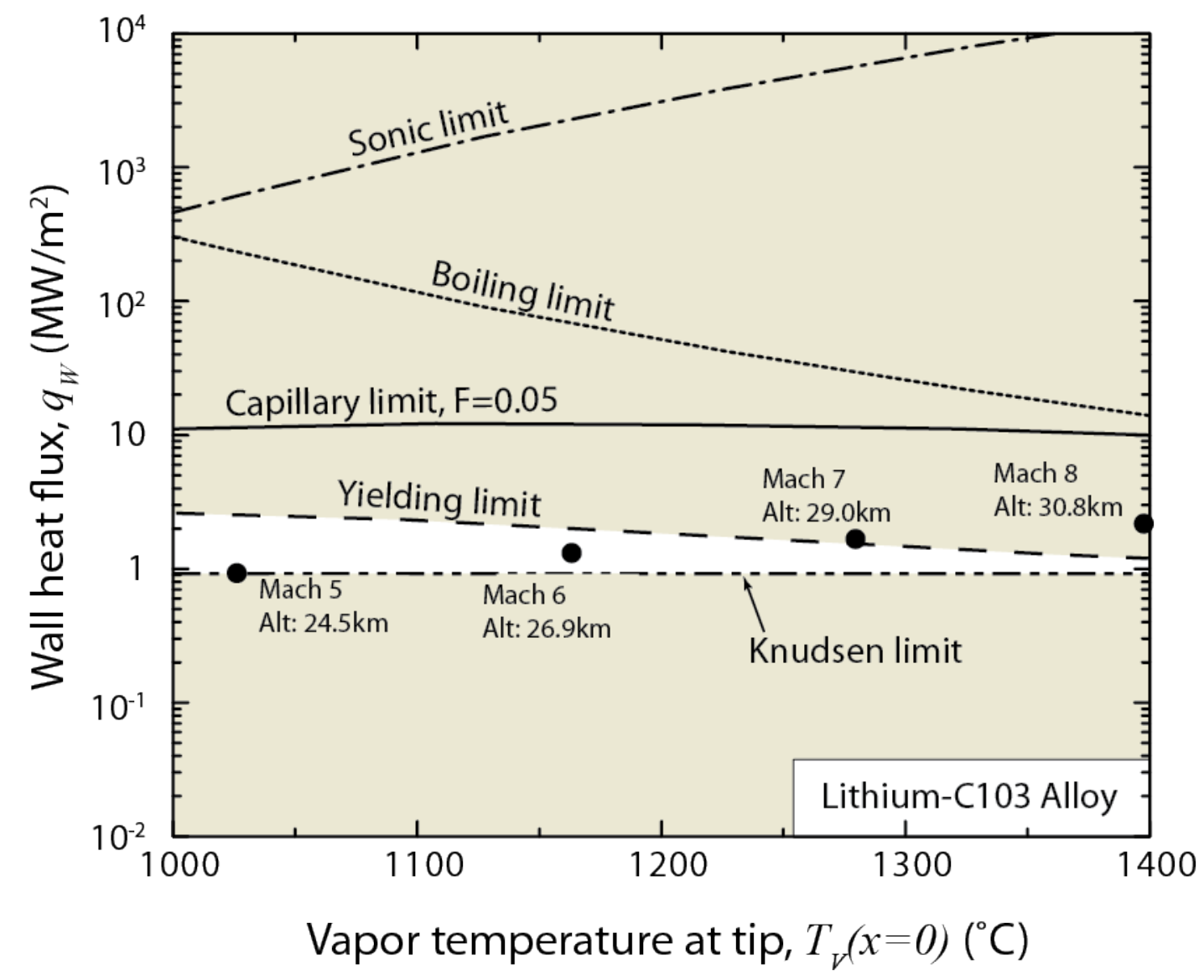

Figure 8.7. Heat transport limits map for a C103/lithium system. The white area delineates the operating window. 


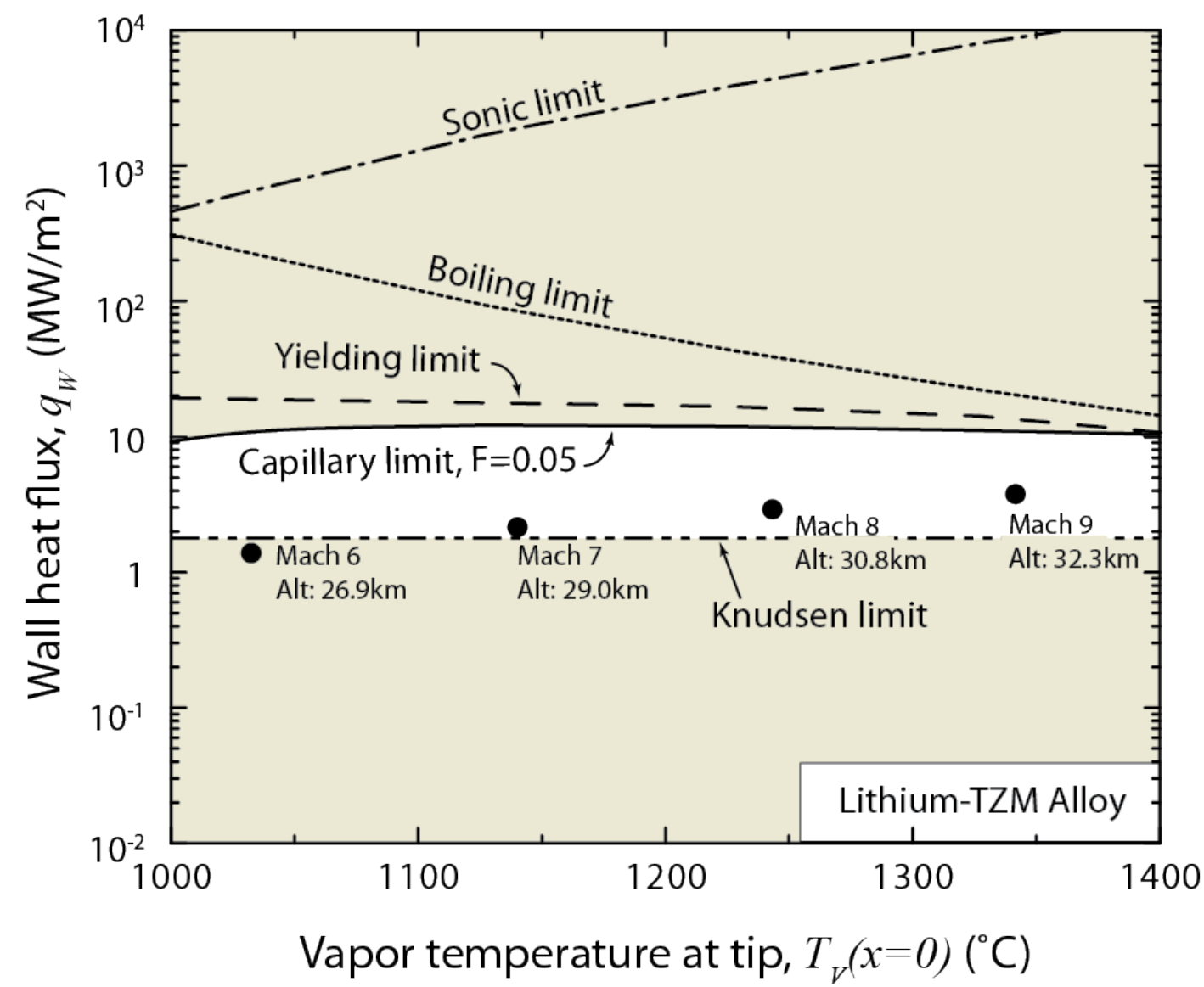

Figure 8.8. Heat transport limits map for a TZM/lithium system. The white area delineates the operating window. 
Table 8.2. Design values for hypersonic leading edges of three case alloy/working fluid combinations. $R_{L E}=3 m m$ and $\varphi=6^{\circ}$

\begin{tabular}{cccc}
\hline & IN718/Sodium & $\begin{array}{c}\text { C103/Lithium } \\
\text { Mach5 }(24.5 \mathrm{~km}) \\
\text { to Mach 7 } \\
(29.0 \mathrm{~km})\end{array}$ & $\begin{array}{c}\text { TZM/Lithium } \\
\text { Mach 7 (29.0km) } \\
\text { (Mach number and altitude) } \\
(32.3 \mathrm{~km})\end{array}$ \\
\hline \hline Design length, $L_{\text {flat }}(\mathrm{mm})$ & Mach 5 $(24.5 \mathrm{~km})$ & 20 & 41.15 \\
Wall thickness, $b_{\text {wall }}(\mathrm{mm})$ & 1.59 & 1.59 & 1.59 \\
Wick thickness, $b_{\text {wick }}(\mathrm{mm})$ & 0.71 & 0.71 & 0.71 \\
Wick pore radius, $R_{P}(\mu \mathrm{m})$ & 10 & 225 & 225 \\
Wick permeability, $K\left(\mathrm{~m}^{2}\right)$ & $7.74 \times 10^{-9}$ & $7.74 \times 10^{-9}$ & $7.74 \times 10^{-9}$ \\
Wick porosity, $\epsilon$ & 0.97 & 0.97 & 0.97 \\
\hline
\end{tabular}




\section{Chapter 9. Conclusion}

This study explored the potential of passive, structurally efficient, high thermal conductance heat spreaders for managing the intense heat that impinges upon the leading edges of hypersonic vehicles. A methodology for their design has been established which considers the influence of design parameters (case wall thickness and condenser length) on the maximum (tip) temperature, isothermal operating temperature, and thermally-induced stress in the tip's wall. As part of this methodology, analytical expressions have been developed to predict the limits of heat transport which at hypersonic flight enthalpies. The models have been experimentally evaluated under simulated hypersonic heat fluxes. It has been found that:

- The relationship between geometric design parameters (condenser length, wall thickness) and operating conditions (wall temperature, maximum [tip] temperature, and thermallyinduced tip stress) can be succinctly presented as a design map given a case alloy, working 
fluid, and flight parameters (Mach number and altitude). The design map delineates the boundary for workable designs so long as the heat transport limits are not encountered. Therefore, a comprehensive design approach using both design and limits maps is necessary.

- A capillary limit model which incorporates (1) pressure drops associated with the change of phase (evaporation and condensation), and (2) active and inactive areas of the liquid-vapor interface participating in evaporation and condensation processes predicts a lower operating limit than the traditional models which do not account for these effects and is more in line with experimental results reported in the literature for cylindrical heat pipes.

- Analytical models predict yielding of the wall material at the leading edge tip when a thermal stress, arising from the strain mismatch generated by large through-thickness thermal gradients, exceeds the material's yield strength. This criterion was formulated as a previously unidentified performance limit, referred to here as the "yielding limit".

- For the fluid, wick, and alloy case material combinations examined here, the predicted sonic and boiling limits are well above the capillary and yielding limits and therefore unlikely to inhibit heat spreader operation.

- A low temperature, proof-of-concept leading edge heat spreader (water-SS304) lowered maximum tip temperatures (up to 43\%) over an identical system without fluid and exhibited isothermal operation. The temperature predictions of the analytical design map and F.E. model were validated, showing good agreement with experimental results. 
- A high temperature, leading edge heat spreader (sodium-IN718 system), tested near Mach 5 enthalpies, lowered maximum tip temperatures (15\%) and axial thermal gradients (33\% on average) but did not achieve a constant temperature profile and exhibited tip temperatures higher than those predicted by the design model.

- By virtue of a heat balance between the condensing surface and the ambient, the length of the heat spreader dictates the vapor operating temperature. This operating temperature must exceed the transition (Knudsen) temperature for the efficient redistribution of heat by convective processes over the entire condenser section, making the design length a critical parameter for isothermal operation.

- If isothermal operation is a design objective, the Knudsen temperature should be included on the limits map and considered during the heat spreader's design.

- It is not necessary for the leading edge to operate isothermally in order to achieve a substantial decrease in tip temperature.

- Using the design methodology:

(i) a sodium-IN718 system has been designed for the heat fluxes of low-altitude Mach 5 (24.5km) flight;

(ii) a lithium-C103 system has been designed for Mach $5(24.5 \mathrm{~km})$ to Mach 7 $(29.0 \mathrm{~km})$ 
(iii) a lithium-TZM system has been designed for Mach 7 (29.0km) flight to over Mach $9(32.3 \mathrm{~km})$. 


\section{References}

[1] T. A. Heppenheimer and N. A. and S. Administration, Facing the Heat Barrier: A History of Hypersonics. Government Printing Office, 2009.

[2] J. D. Anderson, "Aerothermodyanmics: A tutorial discussion," in Thermal structures and materials for high-speed flight, E. A. Thornton, Ed. American Institute of Aeronautics and Astronautics, 1992.

[3] J. D. Anderson, Hypersonic and high temperature gas dynamics. McGraw-Hill, 1989.

[4] E.-H. Hirschel, Basics of aerothermodynamics. Springer, 2005.

[5] C. Peebles, Road to Mach 10: lessons learned from the X-43A flight research program. American Institute of Aeronautics and Astronautics, 2008.

[6] D. V. Wie, S. D’Alessio, and M. White, "Hypersonic Airbreathing Propulsion," Johns Hopkins APL Technical Digest, vol. 26, no. 4, pp. 430-437, 2005.

[7] R. T. Voland, L. D. Huebner, and C. R. McClinton, "X-43A Hypersonic vehicle technology development," Acta Astronautica, vol. 59, no. 1-5, pp. 181-191, 2006.

[8] P. H. Oosthuizen and W. E. Carscallen, Compressible fluid flow. McGraw-Hill, 1997.

[9] J. Arrington and J. Jones, "Shuttle Performance: Lessons Learned," in NASA Conference Publication 2283. 
[10] K. Bowcutt, "Private communication."

[11] R. L. Dotts, H. . Battley, J. T. Hughes, and J. T. Neuenschwander, "Shuttle Orbiter Reusuable Surface Insulation Thermal Performance," in Entry vehicle heating and thermal protection systems: Space Shuttle, Solar Starprobe, Jupiter Galileo Probe, vol. 85, P. E. Bauer, H. E. Collicott, A. I. of A. and Astronautics, and A. S. of M. Engineers, Eds. American Institute of Aeronautics and Astronautics, 1983.

[12] D. B. Paul, C. L. Clay, B. Harber, H. Croop, D. Glass, and S. Scotti, "Extreme Environment Structures," in Structures technology for future aerospace systems, A. K. Noor, Ed. AIAA, 2000.

[13] M. Opeka, I. Talmy, and J. Zaykoski, "Oxidation-based materials selection for 2000 C+ hypersonic aerosurfaces: theoretical considerations and historical experience," Journal of materials science, vol. 39, no. 19, pp. 5887-5904, 2004.

[14] M. F. Ashby, H. Shercliff, and D. Cebon, Materials: Engineering, Science, Processing and Design. Elsevier, 2009.

[15] J. P. Holman, Heat transfer. McGraw-Hill, 2002.

[16] G. V. Candler, "High-Temperature Effects in Hypersonic Flight," Encylopedia of Aerospace Engineering, 2010.

[17] J. Thoemel, O. Chazot, and P. Barbante, "Aspects of advanced catalysis modeling for hypersonic flows," in Proceedings of the Summer Program, 2008.

[18] L. Gong, W. L. Ko, R. D. Quinn, and A. R. Center, Thermal response of space shuttle wing during reentry heating. National Aeronautics and Space Administration, Ames Research Center, Dryden Flight Research Facility, 1984.

[19] D. E. Glass, "Ceramic matrix composite (CMC) thermal protection systems (TPS) and hot structures for hypersonic vehicles," in 15th AIAA International Space Planes and Hypersonic Systems and Technologies Conference, 2008.

[20] J. Lane, "Design Processes and Criteria for the X-51A Flight Vehicle Airframe," Nov. 2007.

[21] T. Jackson, “USA Applied Hypersonics,” Bremen, Germany, 19-Oct-2009.

[22] "Fully Integrated Scramjet Missile Engine Tested at Mach 6.5," www.navy.mil, 06Jun-2002. 
[23] D. Van Wie, D. Drewry, D. King, and C. Hudson, "The hypersonic environment: Required operating conditions and design challenges," Journal of materials science, vol. 39, no. 19, pp. 5915-5924, 2004.

[24] A. Martellucci and T. B. Harris, "An assessment of key aerothermal issues for the structural design of high speed vehicles," in 1st Thermal Structures Conference, Charlottesville, VA, Proceedings, 1990.

[25] S. P. Schneider, "Effects of Roughness on Hypersonic Boundary-Layer Transition," Journal of Spacecraft and Rockets, vol. 45, pp. 193-209, Mar. 2008.

[26] H. N. Kelly and M. L. Blosser, "Active cooling from the sixties to NASP," NASA STI/Recon Technical Report N, vol. 94, p. 37541, Jul. 1994.

[27] E. P. Scala, "A Brief History of Composites in the U. S.-The Dream and the Success," JOM, vol. 48, no. 2, pp. 45-48, 1996.

[28] C. Yaws, Yaw's Critical Property Data for Chemical Engineers and Chemists. Knovel, 2012.

[29] L. Steg and H. Lew, "Hypersonic Ablation,” May 1962.

[30] D. M. Curry, J. A. Cunningham, and J. R. Frahm, "Space Shuttle Orbiter Leading Edge Thermal Performance," in Entry Vehicle Heating and Thermal Protection Systems: Space Shuttle, Solar Starprobe, Jupiter Galileo Probe, vol. 85, P. E. Bauer and H. E. Collicott, Eds. AIAA, 1983.

[31] B. Laub and E. Venkatapathy, "Thermal protection system technology and facility needs for demanding future planetary missions," presented at the International Workshop on Planetary Probe Atmospheric Entry and Descent Trajectory Analysis and Science, Lisbon, Portugal, 2003.

[32] H. Rivers and D. Glass, "Advances in Hot Structure Development," in Thermal Protection Systems and Hot Structures, 2006.

[33] C. J. Camarda, "Analysis and radiant heating tests of a heat-pipe-cooled leading edge,” NASA Tech Note D-8468, 1977.

[34] C. J. Camarda and R. V. Masek, "Design, Analysis, and Tests of a Shuttle-Type Heat-Pipe-Cooled Leading Edge," Journal of Spacecraft and Rockets, vol. 18, no. 1, pp. 71$78,1981$.

[35] D. E. Glass, C. J. Camarda, M. A. Merrigan, and J. T. Sena, "Fabrication and testing of Mo-Re heat pipes embedded in carbon/carbon," Journal of spacecraft and rockets, vol. 36, no. 1, pp. 79-86, 1999. 
[36] D. Glass and C. Carmada, "Preliminary thermal/structural analysis of a carboncarbon/refractory-metal heat-pipe-cooled wing leading edge," in 1st Thermal Structures Conference, Charlottesville, VA, 1990, pp. 241-257.

[37] G. W. Sutton, “The Initial Development of Ablation Heat Protection, An Historical Perspective," Journal of Spacecraft and Rockets, vol. 19, pp. 3-11, Jan. 1982.

[38] J. H. Lundell, "Spallation of the Galileo Probe Heat Shield," in Entry vehicle heating and thermal protection systems: Space Shuttle, Solar Starprobe, Jupiter Galileo Probe, vol. 85, P. E. Bauer and H. E. Collicott, Eds. AIAA and ASM, 1983.

[39] Barton C. Hacker, "Adventures in Research: A History of Ames Research Center, 1940-1965," Technology and Culture, vol. 12, no. 4, pp. 669-671, Oct. 1971.

[40] E. Venkatapathy, B. Laub, G. J. Hartman, J. O. Arnold, M. J. Wright, and G. A. Allen Jr., "Thermal protection system development, testing, and qualification for atmospheric probes and sample return missions: Examples for Saturn, Titan and Stardusttype sample return," Advances in Space Research, vol. 44, no. 1, pp. 138-150, Jul. 2009.

[41] H. K. Tran, C. E. Johnson, D. J. Rasky, F. C. L. Hui, M.-T. Hsu, T. Chen, Y. K. Chen, D. Paragas, and L. Kobayashi, "Phenolic Impregnated Carbon Ablators (PICA) as Thermal Protection Systems for Discovery Missions," Nasa Technical Memorandum, no. April, p. 74, 1997.

[42] H. K. Tran, W. D. Henline, S. H. Ming-ta, D. J. Rasky, and S. R. Riccitiello, Lowdensity resin impregnated ceramic article having an average density of 0.15 to $0.40 \mathrm{~g} / \mathrm{cc}$. Google Patents, 1996.

[43] H. K. Tran, W. D. Henline, S. H. Ming-ta, D. J. Rasky, and S. R. Riccitiello, Lowdensity resin impregnated ceramic article and method for making the same. Google Patents, 1997.

[44] T. H. Squire and J. Marschall, "Material property requirements for analysis and design of UHTC components in hypersonic applications," Journal of the European Ceramic Society, vol. 30, no. 11, pp. 2239-2251, 2010.

[45] M. D. Novak and C. G. Levi, "Oxidation and volatilization of silicide coatings for refractory niobium alloys,” American Society of Mechanical Engineers Paper, vol. 42908, 2007.

[46] M. Z. Alam, B. Venkataraman, B. Sarma, and D. K. Das, "MoSi2 coating on Mo substrate for short-term oxidation protection in air," Journal of Alloys and Compounds, vol. 487, no. 1-2, pp. 335-340, Nov. 2009. 
[47] M. J. Donachie and S. J. Donachie, Superalloys: a technical guide. ASM International, 2002.

[48] L. Ronquillo, "The history of heat shields for manned space flight," in International Astronautical Federation, International Astronautical Congress, 35 th, Lausanne, Switzerland, 1984.

[49] D. R. Jenkins, Space Shuttle: The History of the National Space Transportation System. Voyageur Press, 2007.

[50] Granta Design, CES Material Selection Software. .

[51] J. D. Walker, "From Columbia to Discovery: Understanding the impact threat to the space shuttle," International Journal of Impact Engineering, vol. 36, no. 2, pp. 303-317, 2009.

[52] C. Ohlhorst, D. Glass, W. Bruce, M. Lindell, W. Vaughn, R. Smith, F. V. SAIC, C. Hogenson, T. ATK-GASL, and T. Kowbel, "Development Of X-43 A Mach 10 Leading Edges," in 56 th International Astronautical Congress; Fukuoka; Japan, 2005.

[53] N. S. Jacobson and D. M. Curry, "Oxidation microstructure studies of reinforced carbon/carbon,” Carbon, vol. 44, no. 7, pp. 1142-1150, Jun. 2006.

[54] V. Z. Shemet, A. P. Pomytkin, and V. S. Neshpor, "High-temperature oxidation behaviour of carbon materials in air," Carbon, vol. 31, no. 1, pp. 1-6, 1993.

[55] N. S. Jacobson, D. J. Roth, R. W. Rauser, J. D. Cawley, and D. M. Curry, "Oxidation through coating cracks of SiC-protected carbon/carbon," Surface and Coatings Technology, vol. 203, no. 3-4, pp. 372-383, Nov. 2008.

[56] S.-Q. Guo, "Densification of $\mathrm{ZrB} 2$-based composites and their mechanical and physical properties: A review," Journal of the European Ceramic Society, vol. 29, no. 6, pp. 995-1011, Apr. 2009.

[57] E. Wuchina, M. Opeka, S. Causey, K. Buesking, J. Spain, A. Cull, J. Routbort, and F. Guitierrez-Mora, "Designing for ultrahigh-temperature applications: The mechanical and thermal properties of $\mathrm{HfB} 2, \mathrm{HfCx}, \mathrm{HfNx}$ and $\mathrm{Hf}(\mathrm{N})$," Journal of Materials Science, vol. 39, pp. 5939-5949, Oct. 2004.

[58] W. L. Vaughn and H. G. Maahs, "Active-to-Passive Transition in the Oxidation of Silicon Carbide and Silicon Nitride in Air," Journal of the American Ceramic Society, vol. 73, no. 6, pp. 1540-1543, Jun. 1990. 
[59] W. Kowbel, Y. Huang, and H. Tsou, "Effect of boron ion implantation on the oxidation behavior of three-dimensional carbon-carbon composite," Carbon, vol. 31, no. 2, pp. 355-363, 1993.

[60] D. W. McKee, "Oxidation behavior and protection of carbon/carbon composites," Carbon, vol. 25, no. 4, pp. 551-557, 1987.

[61] S. Ragan and G. T. Emmerson, "The effect of heat-treatment temperature upon the properties of matrix-inhibited carbon-carbon composites," Carbon, vol. 30, no. 3, pp. 339344, 1992.

[62] Y.-J. Lee, H.-J. Joo, and L. R. Radovic, "Preferential distribution and oxidation inhibiting/catalytic effects of boron in carbon fiber reinforced carbon (CFRC) composites," Carbon, vol. 41, no. 13, pp. 2591-2600, 2003.

[63] N. Claussen, "Strengthening strategies for $\mathrm{ZrO} 2$-toughened ceramics at high temperatures," Materials science and engineering, vol. 71, pp. 23-38, 1985.

[64] H. Zhao, C. G. Levi, and H. N. G. Wadley, "Vapor deposited samarium zirconate thermal barrier coatings," Surface and Coatings Technology, vol. 203, no. 20-21, pp. 31573167, Jul. 2009.

[65] H. Zhao, "Low conductivity thermal barrier coatings," University of Virginia, 2009.

[66] M. Gasch and S. Johnson, "Physical characterization and arcjet oxidation of hafnium-based ultra high temperature ceramics fabricated by hot pressing and field-assisted sintering," Journal of the European Ceramic Society, vol. 30, no. 11, pp. 2337-2344, Aug. 2010.

[67] G. Petzow, R. Telle, and R. Danzer, "Microstructural defects and mechanical properties of high-performance ceramics," Materials Characterization, vol. 26, no. 4, pp. 289-302, Jun. 1991.

[68] G. J. Zhang, W. M. Guo, D. W. Ni, and Y. M. Kan, "Ultrahigh temperature ceramics (UHTCs) based on ZrB2 and HfB2 systems: Powder synthesis, densification and mechanical properties," in Journal of Physics: Conference Series, 2009, vol. 176.

[69] A. Spring, Wei-Ming Guo, Guo-Jun Zhang, Pei-Ling Wang, and V. D. Krstic, "Fabrication and Characterization of $\mathrm{ZrB}_{2}$-Based Ceramic Using Synthesized $\mathrm{ZrB}_{2}-\mathrm{LaB}_{6}$ Powder.," Journal of the American Ceramic Society, vol. 91, no. 8, pp. 2763-2765, 2008.

[70] F. Peng and R. F. Speyer, "Oxidation resistance of fully dense ZrB2 with SiC, TaB2, and TaSi2 additives," Journal of the American Ceramic Society, vol. 91, no. 5, pp. 1489-1494, 2008. 
[71] X. Zhang, P. Hu, J. Han, L. Xu, and S. Meng, "The addition of lanthanum hexaboride to zirconium diboride for improved oxidation resistance," Scripta Materialia, vol. 57, no. 11, pp. 1036-1039, 2007.

[72] G. P. Peterson, An introduction to heat pipes: modeling, testing, and applications. Wiley-Interscience, 1994.

[73] A. Faghri, Heat pipe science and technology. Taylor \& Francis, 1995.

[74] D. A. Reay, P. A. Kew, P. A. Kew, and P. D. Dunn, Heat pipes. ButterworthHeinemann, 2006.

[75] D. E. Glass, C. J. Camarda, M. A. Merrigan, J. Sena, and R. S. Reid, "Fabrication and testing of a leading-edge-shaped heat pipe," Journal of Spacecraft and Rockets, vol. 36, no. 6, pp. 921-923, 1999.

[76] L. T. Clark and G. S. Glenn, "Design analysis and testing of liquid metal heat pipes for application to hypersonic vehicles," AIAA, Thermophysics, Plasmadynamics and Lasers Conference, vol. -1, Jun. 1988.

[77] N. Wicks and J. W. Hutchinson, "Optimal truss plates," International Journal of Solids and Structures, vol. 38, no. 30-31, pp. 5165-5183, 2001.

[78] H. N. G. Wadley, "Multifunctional periodic cellular metals," Philosophical Transactions of the Royal Society A: Mathematical, Physical and Engineering Sciences, vol. 364, no. 1838, p. 31, 2006.

[79] H. N. G. Wadley, N. A. Fleck, and A. G. Evans, "Fabrication and structural performance of periodic cellular metal sandwich structures," Composites Science and Technology, vol. 63, no. 16, pp. 2331-2343, 2003.

[80] J. H. Lim and K. J. Kang, "Mechanical behavior of sandwich panels with tetrahedral and Kagome truss cores fabricated from wires," International journal of solids and structures, vol. 43, no. 17, pp. 5228-5246, 2006.

[81] D. T. Queheillalt and H. N. G. Wadley, "Cellular metal lattices with hollow trusses," Acta Materialia, vol. 53, no. 2, pp. 303-313, 2005.

[82] Z. Wei, V. S. Deshpande, A. G. Evans, K. P. Dharmasena, D. T. Queheillalt, H. N. G. Wadley, Y. V. Murty, R. K. Elzey, P. Dudt, Y. Chen, D. Knight, and K. Kiddy, "The resistance of metallic plates to localized impulse," Journal of the Mechanics and Physics of Solids, vol. 56, no. 5, pp. 2074-2091, May 2008.

[83] A. Basiulis and C. Camarda, "Design, fabrication and test of liquid metal heat-pipe sandwich panels," NASA STI/Recon Technical Report N, vol. 83, p. 22541, 1983. 
[84] D. T. Queheillalt, G. Carbajal, G. Peterson, and H. N. G. Wadley, "A multifunctional heat pipe sandwich panel structure," International Journal of Heat and Mass Transfer, vol. 51, no. 1-2, pp. 312-326, 2008.

[85] H. N. G. Wadley, "Private communication."

[86] M. A. Merrigan, J. Sena, and D. E. Glass, "Evaluation of a sodium/Hastelloy-X heat pipe for wing leading edge cooling," Heat transfer-Houston 1996, pp. 333-341, 1996.

[87] B. Boman and T. Elias, "Tests on a sodium/Hastelloy $\mathrm{X}$ wing leading edge heat pipe for hypersonic vehicles," in AIAA and ASME, 5th Joint Thermophysics and Heat Transfer Conference, 1990.

[88] C. C. Silverstein, A feasibility study of heat-pipe-cooled leading edges for hypersonic cruise aircraft. National Aeronautics and Space Administration, 1971.

[89] H. G. Cowgill, "Sodium Corrosion of Alloy 718 at 649C," Department of Energy, TI85 026707, 1984.

[90] J. H. Stang, E. M. Simons, J. A. DeMastry, and J. M. Genco, "Compatibility of Liquid and Vapor Alkali Metals with Construction Materials," DMIC 227, Apr. 1966.

[91] C. E. Sessions and J. H. DeVan, "Effect of Oxygen, Heat Treatment, and Test Temperature on the compatibility of Several Advanced Refractory Alloys with Lithium," ORNL4430, Apr. 1971.

[92] A. Faghri and C. Harley, "Transient lumped heat pipe analyses," Heat Recovery Systems and CHP, vol. 14, no. 4, pp. 351-363, Jul. 1994.

[93] F. M. White, Fluid mechanics. WCB/McGraw-Hill, 1999.

[94] C. A. Steeves, M. Y. He, S. D. Kasen, L. Valdevit, H. N. G. Wadley, and A. G. Evans, "Feasibility of metallic structural heat pipes as sharp leading edges for hypersonic vehicles," Journal of Applied Mechanics, vol. 76, p. 031014, 2009.

[95] W. H. Heiser and D. T. Pratt, Hypersonic airbreathing propulsion. AIAA, 1994.

[96] U. COESA, "Standard Atmosphere 1976," US Government Printing Office, Washington, DC, 1976.

[97] F. M. White, Viscous fluid flow. McGraw-Hill, 1991.

[98] L. Lees, "Laminar heat transfer over blunt-nosed bodies at hypersonic flight speeds," AIAA Journal, vol. 41, no. 7; Supplement A, pp. 356-366, 2003. 
[99] C. Goyne, R. Stalker, and A. Paull, "Skin-friction measurements in high-enthalpy hypersonic boundary layers," Journal of Fluid Mechanics, vol. 485, no. 25, pp. 1-32, 2003.

[100] F. Livingston, "Oblique shock wave angle charts for a perfect gas," Jet Propulsion Lab., California Inst. of Tech., Pasadena, 1964.

[101] E. W. Cheney and D. R. Kincaid, Numerical mathematics and computing. Cengage Learning, 2007.

[102] S. D. Kasen, "Unpublished research." .

[103] F. Kreith and W. Z. Black, Basic heat transfer. Harper \& Row, 1980.

[104] P. Vinz and C. Busse, "Axial heat transfer limits of cylindrical sodium heat pipes between $25 \mathrm{~W} / \mathrm{cm} 2$ and $15.5 \mathrm{k} \mathrm{W} / \mathrm{cm}$ 2," in Proceedings of 1st International Heat Pipe Conference, Stuttgart, Germany, 1973.

[105] R. Richter and J. M. Gottschlich, "Thermodynamic aspects of heat pipe operation," Journal of Thermophysics and Heat Transfer, vol. 8, pp. 334-340, Apr. 1994.

[106] D. D. Odhekar and D. K. Harris, "Experimental investigation of bendable heat pipes using sintered copper felt wick," in Thermal and Thermomechanical Phenomena in Electronics Systems, 2006.

[107] S. W. Chi, Heat pipe theory and practice: a sourcebook. Hemisphere Pub. Corp., 1976.

[108] V. P. Carey, Liquid-vapor phase-change phenomena. Taylor and Francis, 2008.

[109] H.-J. Butt, K. Graf, and M. Kappl, Physics and Chemistry of Interfaces. John Wiley \& Sons, 2006.

[110] H. Wang, X. Peng, S. V. Garimella, and D. M. Christopher, "Microbubble return phenomena during subcooled boiling on small wires," International Journal of Heat and Mass Transfer, vol. 50, no. 1-2, pp. 163-172, Jan. 2007.

[111] H. Wang, S. V. Garimella, and J. Y. Murthy, "An analytical solution for the total heat transfer in the thin-film region of an evaporating meniscus," International Journal of Heat and Mass Transfer, vol. 51, no. 25-26, pp. 6317-6322, Dec. 2008.

[112] H. Wang, S. V. Garimella, and J. Y. Murthy, "Characteristics of an evaporating thin film in a microchannel," International Journal of Heat and Mass Transfer, vol. 50, no. 19-20, pp. 3933-3942, Sep. 2007. 
[113] J. A. Schonberg, S. DasGupta, and P. C. Wayner Jr., "An augmented Young-Laplace model of an evaporating meniscus in a microchannel with high heat flux," Experimental Thermal and Fluid Science, vol. 10, no. 2, pp. 163-170, Feb. 1995.

[114] S. DasGupta, J. Schonberg, and P. Wayner, "Investigation of an evaporating extended meniscus based on the augmented Young-Laplace equation," ASME Journal of Heat Transfer, vol. 115, pp. 201-201, 1993.

[115] H. Haj-Hariri, "Private communication."

[116] S. S. Panchamgam, A. Chatterjee, J. L. Plawsky, and P. C. Wayner Jr., "Comprehensive experimental and theoretical study of fluid flow and heat transfer in a microscopic evaporating meniscus in a miniature heat exchanger," International Journal of Heat and Mass Transfer, vol. 51, no. 21-22, pp. 5368-5379, Oct. 2008.

[117] J. G. Collier and J. R. Thome, Convective boiling and condensation. Oxford University Press, 1996.

[118] A. Marchand, J. H. Weijs, J. H. Snoeijer, and B. Andreotti, "Why is surface tension a force parallel to the interface?," American Journal of Physics, vol. 79, p. 999, 2011.

[119] W. D. Callister, Materials Science and Engineering: An Introduction. John Wiley \& Sons, 2002.

[120] A. B. Cohen and A. D. Kraus, Advances in thermal modeling of electronic components and systems. Hemisphere Pub. Corp., 1998.

[121] B. Paul, "Compilation of Evaporation Coefficients," American Rocket Society Journal, vol. 32, p. 1321, 1962.

[122] A. I. H. Committee, Metals Handbook. Asm International, 1998.

[123] R. Monazami, "Part I: On the evaporation of menisci in microchannels Part II: A momentum integral model for prediction of steady operation and flooding in thermosyphons," University of Virginia, 2012.

[124] R. W. Johnson, The Handbook of Fluid Dynamics. CRC Press, 1998.

[125] W. M. Rohsenow, J. P. Hartnett, and Y. I. Cho, Handbook of heat transfer. McGrawHill, 1998.

[126] E. K. Levy, "Theoretical Investigation of Heat Pipes Operating at Low Vapor Pressures," Journal of Engineering for Industry, vol. 90, no. 4, p. 547, 1968. 
[127] C. A. Steeves, M. Y. He, L. Valdevit, S. Kasen, H. Haj-Hariri, H. N. G. Wadley, and A. G. Evans, "Metallic Structural Heat Pipes as Sharp Leading Edges for Mach 7 Vehicles," in IMECE2007, Seattle, WA, 2007.

[128] S. D. Kasen, C. Steeves, D. Queheillalt, A. G. Evans, and H. N. G. Wadley, "A heat plate leading edge for hypersonic vehicles," in Proceedings of the ASME International Mechanical Engineering Congress and Exposition, Boston, MA, 2008, pp. 2-6.

[129] J. Tian, T. Kim, T. J. Lu, H. P. Hodson, D. T. Queheillalt, D. J. Sypeck, and H. N. G. Wadley, "The effects of topology upon fluid-flow and heat-transfer within cellular copper structures," International Journal of Heat and Mass Transfer, vol. 47, no. 14-16, pp. 31713186, Jul. 2004.

[130] DuPont, "Nomex 994 Technical Data Sheet.” 2002.

[131] A. J. Neves and M. H. Nazaré, Properties, Growth, and Applications of Diamond. IET, 2001.

[132] R. P. Ananatmulat, S. J. Mayhan, and P. A. White, "Microstructural Characterization of Advanced Ferrous Alloys Exposed to Liquid Sodium," in Tenth Annual Technical Meeting of the International Metallographic Society, Houston, Texas, 1977.

[133] Novamet, “Open Cell Nickel Foam Product Data Sheet.” .

[134] Greene G.A. and Finfrock C.C., "Oxidation of Inconel 718 in Air at High Temperatures," Oxidation of Metals, vol. 55, no. 5/6, pp. 505-521, 2001.

[135] Y. Cao and A. Faghri, "Micro/miniature heat pipes and operating limitations," Journal of Enhanced Heat Transfer, vol. 1, no. 3, 1994.

[136] E. Rathakrishnan, Gas Dynamics. PHI Learning Pvt. Ltd., 2004.

[137] I. Valyi, Atomic and ionic sources. Wiely, 1977.

[138] C. R. Brooks, M. Cash, and A. Garcia, “The Heat Capacity of Inconel 718 from 313 to 1053 deg K," Journal of Nuclear Materials, vol. 78, no. 2, pp. 419-421, 1978.

[139] Allegheny Technologies Inc., “C103 Alloy Product Data Sheet.” 2012.

[140] Special Metals, "Inconel 718 Product Data Sheet." 2007.

[141] T. E. Tietz and J. W. Wilson, Behavior and Properties of Refractory Metals. Stanford University Press, 1965. 
[142] ITER Council, "Final Report of the ITER Engineering Design Activities," ITER EDA No. 21, 2001.

[143] U. S. D. of Defense, Metallic materials and elements for aerospace vehicle structures. U.S. Dept. of Defense, 1994.

[144] R. W. Ohse, Ed., Handbook of thermodynamic and transport properties of alkali metals. Blackwell Scientific, 1985.

[145] M. A. Saad, Compressible Fluid Flow. Prentice-Hall, 1985.

[146] R. J. Moffat, "Uncertainty Analysis," in Thermal Measurements in Electronics Cooling, K. Azar, Ed. CRC Press, 1997.

[147] Extech Instruments, “Extech 430 User’s Guide.” Aug-2007.

[148] Omega Engineering, "Product Data Sheet." . 


\section{Appendix A. Thermo-mechanical properties for SS-304, IN718,C-103, and TZM}


Stainless Steel AISI 304 mechanical properties (taken as constant with temperature)

\begin{tabular}{cc}
\hline Property & Value [28], [122] \\
\hline \hline Young's modulus, $E$ & $195 \mathrm{GPa}$ \\
Yield strength, $\sigma_{y}$ & $215 \mathrm{MPa}$ \\
Thermal expansion coefficient, $\alpha$ & $17.3 \mu \mathrm{m} / \mathrm{m}^{\circ} \mathrm{C}$ \\
Poisson's ratio, $\nu$ & 0.33 \\
Thermal conductivity, $k$ & $16.2 \mathrm{~W} / \mathrm{mK}$ \\
\hline
\end{tabular}


Inconel 718, C-103, and TZM Temperature-dependent heat capacity

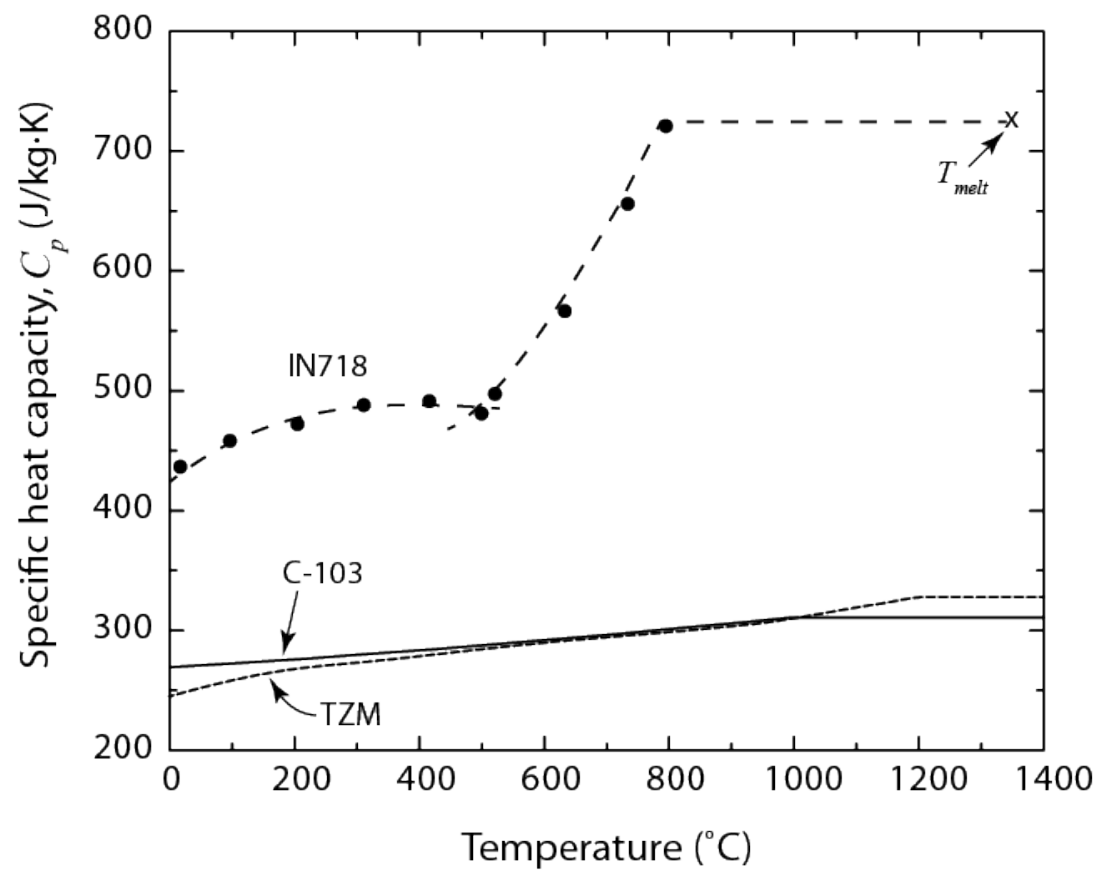

Figure A.1 Heat capacity temperature dependence. 


\begin{tabular}{|c|c|c|c|c|c|}
\hline Alloy & & Equation $\left(T\right.$ in ${ }^{\circ} \mathrm{C}, C_{p}$ in $\left.\mathrm{J} / \mathrm{kgK}\right)$ & & & Reference(s) \\
\hline IN718 & & $C_{p}=\left\{\begin{array}{c}526.727-0.0476148 \mathrm{~T}-8.92138 \times \frac{10^{6}}{(273.15+\mathrm{T})^{2}} \\
2.71 \frac{(-133.249+\mathrm{T})\left(399965 .-711.241 \mathrm{~T}+\mathrm{T}^{2}\right)}{(273.15+\mathrm{T})^{2}} \\
725\end{array}\right.$ & $\begin{array}{c}T \leq 507^{\circ} \mathrm{C} \\
507<T \leq 787^{\circ} \mathrm{C} \\
T>787^{\circ} \mathrm{C}\end{array}$ & & {$[138]$} \\
\hline C-103 & & $C_{p}=\left\{\begin{array}{c}269.053+0.0323504 \mathrm{~T}+9.79386 \times 10^{-6} \mathrm{~T}^{2} \\
311\end{array}\right.$ & $\begin{array}{l}\mathrm{T} \leq 1000^{\circ} \mathrm{C} \\
\mathrm{T}>1000^{\circ} \mathrm{C}\end{array}$ & & [139] \\
\hline $\mathrm{TZM}^{15}$ & $C_{p}=\{$ & $\left\{\begin{array}{c}-15.0691+2.12289 \mathrm{~T}-0.00666008 \mathrm{~T}^{2}+0.0000109688 \mathrm{~T}^{3}-9 \\
+4.41531 \times 10^{-12} \mathrm{~T}^{5}-8.00137 \times 10^{-16} \mathrm{~T}^{6} \\
328\end{array}\right.$ & $.73604 \times 10^{-9} \mathrm{~T}^{4}$ & $\begin{array}{l}T \leq 1227^{\circ} \mathrm{C} \\
T>1227^{\circ} \mathrm{C}\end{array}$ & {$[28]$} \\
\hline
\end{tabular}

${ }^{15}$ Taken as molybdenum. 
Inconel 718, C-103, and TZM Temperature-dependent yield strength

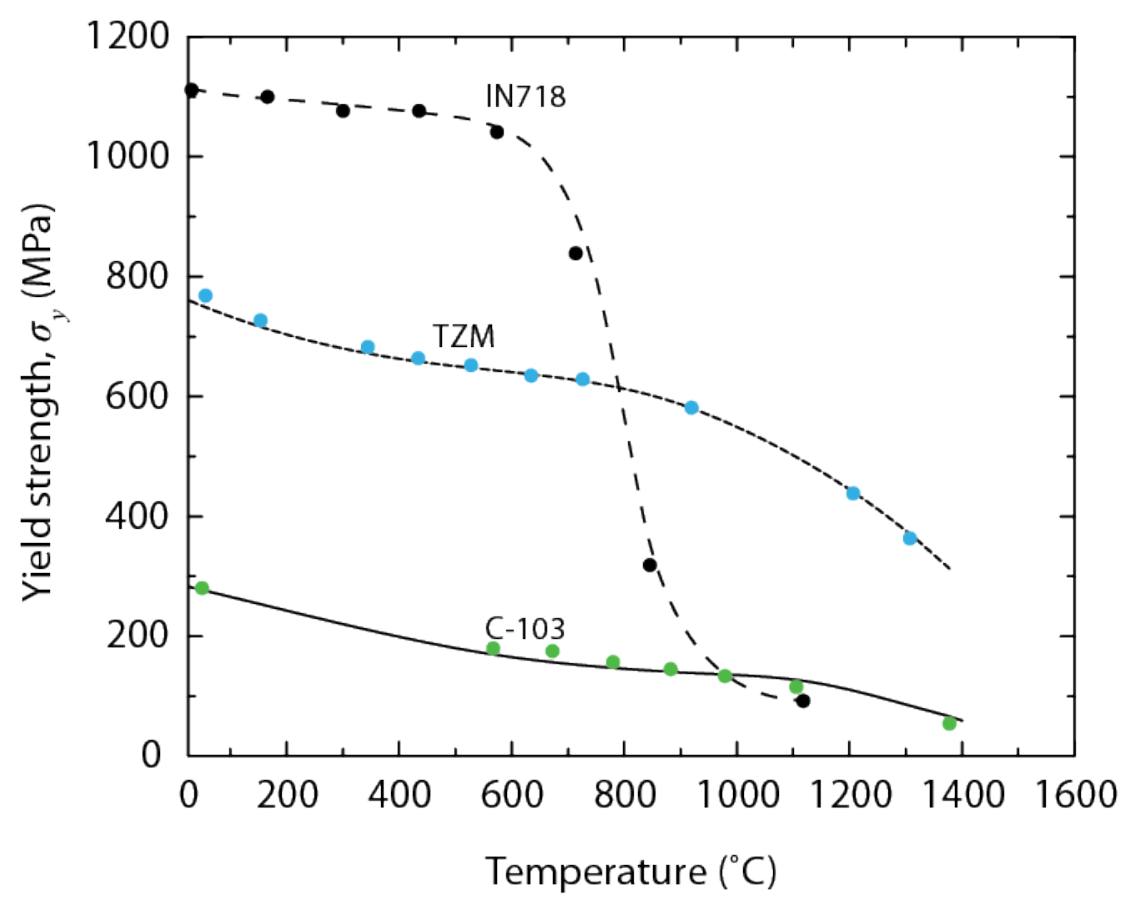

Figure A.2 Yield strength temperature dependence. 
Table A. 1. Temperature dependent yield strength of IN718 [140].

\begin{tabular}{cc}
\hline $\begin{array}{c}\text { Temperature, } T \\
\left({ }^{\circ} \mathrm{C}\right)\end{array}$ & $\begin{array}{c}\text { Yield strength, } \sigma_{y} \\
(\mathrm{MPa})\end{array}$ \\
\hline \hline 93 & 1170 \\
204 & 1100 \\
316 & 1090 \\
427 & 1070 \\
538 & 1070 \\
649 & 1030 \\
760 & 827 \\
871 & 310 \\
982 & 138 \\
1093 & 103
\end{tabular}

Table A. 2. Temperature dependent yield strength of C103 [139].

\begin{tabular}{cc}
\hline Temperature, $T\left({ }^{\circ} \mathrm{C}\right)$ & $\begin{array}{c}\text { Yield strength, } \sigma_{y} \\
(\mathrm{MPa})\end{array}$ \\
\hline \hline 25 & 269 \\
537 & 158 \\
760 & 138 \\
870 & 124 \\
1093 & 124 \\
1370 & 55 \\
&
\end{tabular}

Table A. 3. Temperature dependent yield strength of TZM [28].

\begin{tabular}{cc}
\hline $\begin{array}{c}\text { Temperature, } T \\
\left({ }^{\circ} \mathrm{C}\right)\end{array}$ & $\begin{array}{c}\text { Yield strength, } \sigma_{y} \\
(\mathrm{MPa})\end{array}$ \\
\hline \hline 27 & 759.589 \\
127 & 721.36 \\
327 & 673.5 \\
427 & 658.86 \\
527 & 647.08 \\
627 & 635.65 \\
727 & 622.07 \\
927 & 578.48 \\
1227 & 434.41 \\
1327 & 355.41 \\
1477 & 190
\end{tabular}


Inconel 718, C-103, and TZM Temperature-dependent elastic modulus

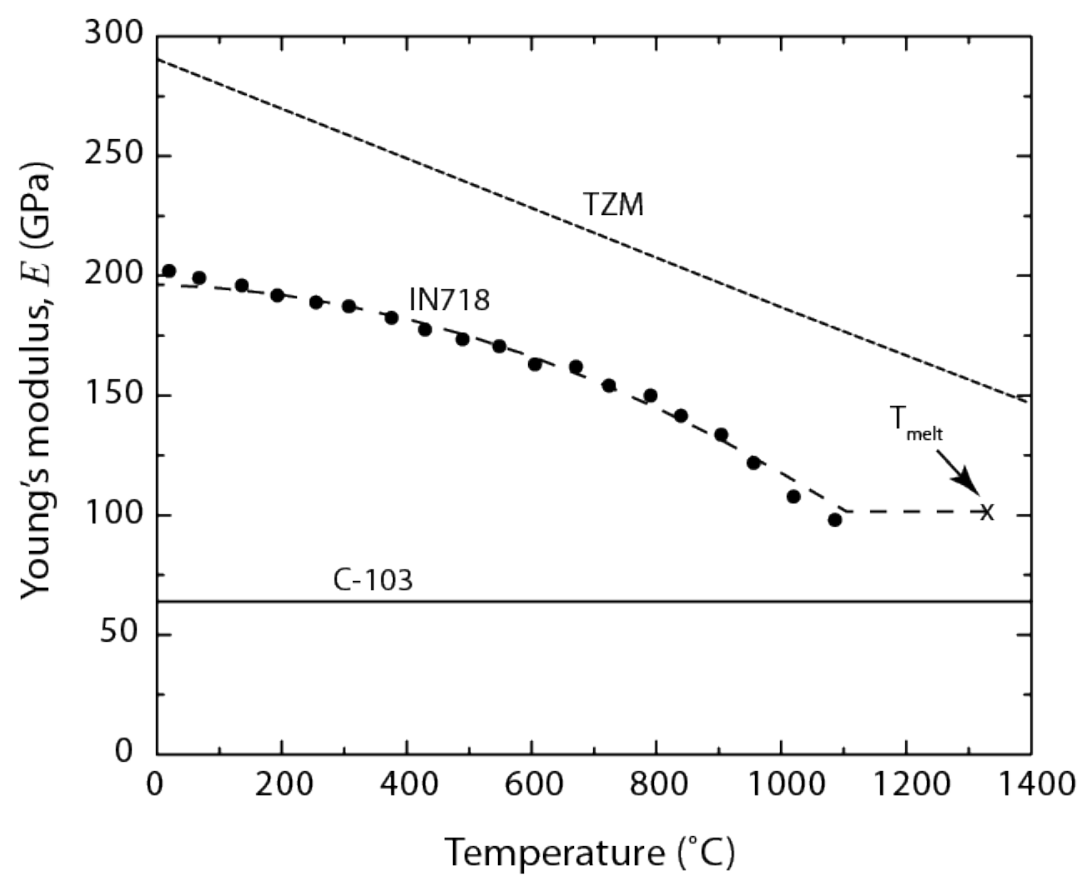

Figure A.3 Young's modulus temperature dependence. 


\begin{tabular}{|c|c|c|c|}
\hline Alloy & Equation $\left(T\right.$ in ${ }^{\circ} \mathrm{C}, E$ in $\left.\mathrm{GPa}\right)$ & & Reference $(s)$ \\
\hline IN718 & $E=\left\{\begin{array}{c}196.413-0.0076461 T-0.000071032 T^{2} \\
103\end{array}\right.$ & $\begin{array}{l}T \leq 1093^{\circ} \mathrm{C} \\
T>1093^{\circ} \mathrm{C}\end{array}$ & {$[140]$} \\
\hline C-103 & $\mathrm{E}=64$ & & [139] \\
\hline TZM & $E=290.497-0.10369 T$ & & [141], [142] \\
\hline
\end{tabular}


Inconel 718, C-103, and TZM Temperature-dependent thermal conductivity

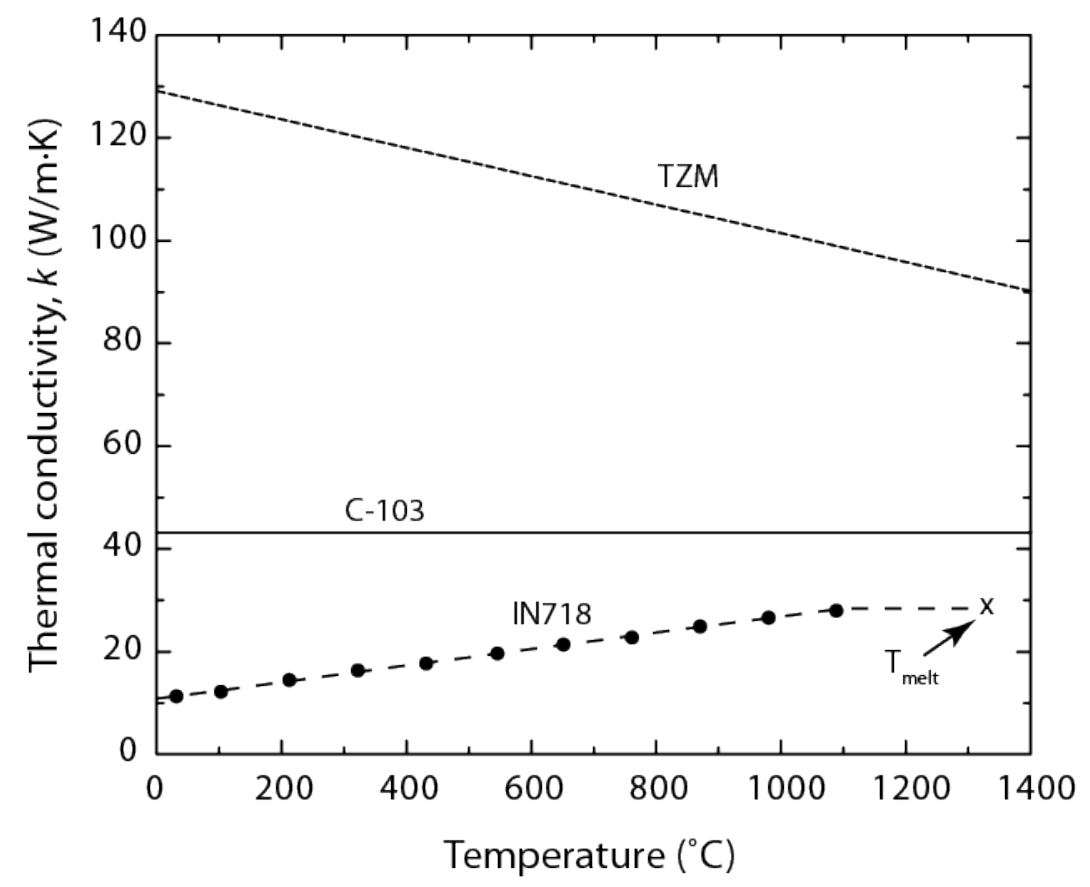

Figure A.4 Thermal conductivity temperature dependence. 


\begin{tabular}{|c|c|c|c|}
\hline Alloy & Equation $\left(T\right.$ in ${ }^{\circ} \mathrm{C}, k$ in $\left.\mathrm{W} / \mathrm{mK}\right)$ & & Reference $(s)$ \\
\hline IN718 & $k=\left\{\begin{array}{c}10.803+0.0162949 T-2.53206 \times 10^{-7} T^{2} \\
28.31\end{array}\right.$ & $\begin{array}{l}, T<1093^{\circ} \mathrm{C} \\
T \geq 1093^{\circ} \mathrm{C}\end{array}$ & [143] \\
\hline C-103 & $k=43.2$ & & [139] \\
\hline TZM & $k=129.133-0.027631 T$ & & [141], [142] \\
\hline
\end{tabular}


Inconel 718, C-103, and TZM thermal expansion coefficient and Poisson's ratio

\begin{tabular}{cccc}
\hline Alloy & $\begin{array}{c}\text { Thermal expansion coefficient, } \alpha \\
\left(\mu \mathrm{m} / \mathrm{m}^{\circ} \mathrm{C}\right)\end{array}$ & Poisson's ratio, $\nu$ & Reference $(\mathrm{s})$ \\
\hline \hline IN718 & $16\left(\right.$ at $\left.800^{\circ} \mathrm{C}\right)$ & 0.32 & {$[140]$} \\
C-103 & $8.10\left(1200^{\circ} \mathrm{C}\right)$ & 0.38 & {$[139]$} \\
TZM & $6.70\left(1227^{\circ} \mathrm{C}\right)$ & 0.32 & {$[141],[142]$} \\
\hline
\end{tabular}




\section{Appendix B. Working fluid properties for water, sodium, and lithium}




\section{Thermophysical properties of water}

From [108]

Molar Mass, $M=0.018 \mathrm{~kg} / \mathrm{mol}$

Heat capacity ratio, $\gamma=1.334$

Accommodation coefficient, $c=0.04$

\begin{tabular}{|c|c|c|c|c|c|c|c|c|c|}
\hline $\begin{array}{c}\text { Vapor } \\
\text { Temperature, } \\
T_{V} \\
\left({ }^{\circ} \mathrm{C}\right)\end{array}$ & $\begin{array}{c}\text { Latent Heat of } \\
\text { Vaporization, } \\
\lambda \\
(\mathrm{J} / \mathrm{kg})\end{array}$ & $\begin{array}{c}\text { Liquid } \\
\text { Density, } \\
\rho_{L} \\
\left(\mathrm{~kg} / \mathrm{m}^{3}\right)\end{array}$ & $\begin{array}{c}\text { Vapor } \\
\text { Density, } \\
\rho_{V} \\
\left(\mathrm{~kg} / \mathrm{m}^{3}\right)\end{array}$ & $\begin{array}{c}\text { Liquid } \\
\text { Thermal } \\
\text { Conductivity, } \\
k_{L} \\
(\mathrm{~W} / \mathrm{mK}) \\
\end{array}$ & $\begin{array}{c}\text { Liquid } \\
\text { Viscosity, } \\
\mu_{L} \\
(\mathrm{~kg} / \mathrm{ms})\end{array}$ & $\begin{array}{c}\text { Vapor } \\
\text { Viscosity, } \\
\mu_{V} \\
(\mathrm{~kg} / \mathrm{ms})\end{array}$ & $\begin{array}{c}\text { Surface } \\
\text { Tension, } \\
\sigma_{L V} \\
(\mathrm{~N} / \mathrm{m})\end{array}$ & $\begin{array}{c}\text { Sound } \\
\text { Velocity, } \\
c \\
(\mathrm{~m} / \mathrm{s})\end{array}$ & $\begin{array}{c}\text { Saturation } \\
\text { Pressure, } \\
P_{V} \\
(\mathrm{~Pa})\end{array}$ \\
\hline 20 & 2448000 & 998 & 0.02 & 0.603 & 0.001 & 0.0000096 & 0.0728 & 423.80 & 2339.20 \\
\hline 40 & 2402000 & 992.1 & 0.05 & 0.63 & 0.00065 & 0.0000104 & 0.07 & 438.00 & 7384.40 \\
\hline 60 & 2359000 & 983.3 & 0.13 & 0.649 & 0.00047 & 0.0000112 & 0.0666 & 451.60 & 19946.00 \\
\hline 80 & 2309000 & 972 & 0.29 & 0.668 & 0.00036 & 0.0000119 & 0.0626 & 464.70 & 47415.00 \\
\hline 100 & 2258000 & 958 & 0.6 & 0.68 & 0.00028 & 0.0000127 & 0.0589 & 477.30 & 101420.00 \\
\hline 120 & 2200000 & 945 & 1.12 & 0.682 & 0.00023 & 0.0000134 & 0.055 & 489.60 & 198670.00 \\
\hline 140 & 2139000 & 928 & 1.99 & 0.683 & 0.0002 & 0.0000141 & 0.0506 & 501.50 & 361500.00 \\
\hline 160 & 2074000 & 909 & 3.27 & 0.679 & 0.00017 & 0.0000149 & 0.0466 & 513.00 & 618140.00 \\
\hline 180 & 2003000 & 888 & 5.16 & 0.669 & 0.00015 & 0.0000157 & 0.0429 & 524.30 & 1002600.00 \\
\hline 200 & 1967000 & 865 & 7.87 & 0.659 & 0.00014 & 0.0000165 & 0.0389 & 535.30 & 1554700.00 \\
\hline
\end{tabular}




\section{Thermophysical properties of sodium}

From [28], [121], [144]

Molar Mass, $M=0.023 \mathrm{~kg} / \mathrm{mol}$

Heat capacity ratio, $\gamma=1.6$

Accommodation coefficient, $c=1.0$

\begin{tabular}{|c|c|c|c|c|c|c|c|c|c|}
\hline $\begin{array}{c}\text { Vapor } \\
\text { Temperature, } \\
T_{V} \\
\left({ }^{\circ} \mathrm{C}\right)\end{array}$ & $\begin{array}{c}\text { Latent Heat of } \\
\text { Vaporization, } \\
\lambda \\
(\mathrm{J} / \mathrm{kg})\end{array}$ & $\begin{array}{c}\text { Liquid } \\
\text { Density, } \\
\rho_{L} \\
\left(\mathrm{~kg} / \mathrm{m}^{3}\right)\end{array}$ & $\begin{array}{c}\text { Vapor } \\
\text { Density, } \\
\rho_{V} \\
\left(\mathrm{~kg} / \mathrm{m}^{3}\right)\end{array}$ & $\begin{array}{c}\text { Liquid } \\
\text { Thermal } \\
\text { Conductivity, } \\
k_{L} \\
(\mathrm{~W} / \mathrm{mK})\end{array}$ & $\begin{array}{c}\text { Liquid } \\
\text { Viscosity, } \\
\mu_{L} \\
(\mathrm{~kg} / \mathrm{ms})\end{array}$ & $\begin{array}{c}\text { Vapor } \\
\text { Viscosity, } \\
\mu_{V} \\
(\mathrm{~kg} / \mathrm{ms})\end{array}$ & $\begin{array}{c}\text { Surface } \\
\text { Tension, } \\
\sigma_{L V} \\
(\mathrm{~N} / \mathrm{m})\end{array}$ & $\begin{array}{c}\text { Sound } \\
\text { Velocity, } \\
c \\
(\mathrm{~m} / \mathrm{s})\end{array}$ & $\begin{array}{c}\text { Saturation } \\
\text { Pressure, } \\
P_{V} \\
(\mathrm{~Pa})\end{array}$ \\
\hline 477 & 4258084 & 837.3 & 0.001307 & 66 & 0.000244 & 0.00001565 & 0.1533 & 587.1 & 343 \\
\hline 527 & 4213326 & 825.1 & 0.003419 & 63.4 & 0.000227 & 0.00001599 & 0.1483 & 603.4 & 949 \\
\hline 577 & 4167699 & 813.1 & 0.007968 & 61.05 & 0.000213 & 0.00001627 & 0.1433 & 619.5 & 2330 \\
\hline 627 & 4121203 & 801.2 & 0.01683 & 58.7 & 0.000201 & 0.00001652 & 0.1383 & 635.3 & 5150 \\
\hline 677 & 4074272 & 789.5 & 0.03278 & 56.55 & 0.00019 & 0.00001674 & 0.1334 & 650.8 & 10500 \\
\hline 727 & 4026038 & 777.8 & 0.05959 & 54.4 & 0.000181 & 0.00001694 & 0.1285 & 666 & 19900 \\
\hline 777 & 3976934 & 766.1 & 0.102 & 52.4 & 0.000173 & 0.00001713 & 0.1236 & 680.9 & 35400 \\
\hline 827 & 3926528 & 754.5 & 0.166 & 50.4 & 0.000166 & 0.00001732 & 0.1187 & 695.6 & 59600 \\
\hline 877 & 3875686 & 742.8 & 0.2624 & 48.6 & 0.000159 & 0.00001751 & 0.1139 & 710 & 96100 \\
\hline 927 & 3823106 & 730.9 & 0.3909 & 46.8 & 0.000153 & 0.00001771 & 0.1091 & 724.1 & 150000 \\
\hline 977 & 3769658 & 719 & 0.5652 & 45.2 & 0.000148 & 0.00001792 & 0.1044 & 738 & 224000 \\
\hline 1027 & 3714905 & 706.8 & 0.7844 & 43.5 & 0.000143 & 0.00001814 & 0.09968 & 752.2 & 322000 \\
\hline
\end{tabular}




\section{Thermophysical properties of lithium}

From [28], [121], [144]

Molar Mass, $M=0.0069 \mathrm{~kg} / \mathrm{mol}$

Heat capacity ratio, $\gamma=1.6$

Accommodation coefficient, $c=1.0$

\begin{tabular}{|c|c|c|c|c|c|c|c|c|c|}
\hline $\begin{array}{c}\text { Vapor } \\
\text { Temperature } \\
, T_{V} \\
\left({ }^{\circ} \mathrm{C}\right)\end{array}$ & $\begin{array}{c}\text { Latent Heat of } \\
\text { Vaporization, } \\
\lambda \\
(\mathrm{J} / \mathrm{kg})\end{array}$ & $\begin{array}{c}\text { Liquid } \\
\text { Density, } \\
\rho_{L} \\
\left(\mathrm{~kg} / \mathrm{m}^{3}\right)\end{array}$ & $\begin{array}{c}\text { Vapor } \\
\text { Density, } \rho_{V} \\
\left(\mathrm{~kg} / \mathrm{m}^{3}\right)\end{array}$ & $\begin{array}{c}\text { Liquid } \\
\text { Thermal } \\
\text { Conductivity } \\
, k_{L} \\
(\mathrm{~W} / \mathrm{mK})\end{array}$ & $\begin{array}{c}\text { Liquid } \\
\text { Viscosity, } \\
\mu_{L} \\
(\mathrm{~kg} / \mathrm{ms})\end{array}$ & $\begin{array}{c}\text { Vapor } \\
\text { Viscosity, } \\
\mu_{V} \\
(\mathrm{~kg} / \mathrm{ms})\end{array}$ & $\begin{array}{c}\text { Surface } \\
\text { Tension, } \\
\sigma_{L V} \\
(\mathrm{~N} / \mathrm{m})\end{array}$ & $\begin{array}{c}\text { Sound } \\
\text { Velocity, } \\
c \\
(\mathrm{~m} / \mathrm{s})\end{array}$ & $\begin{array}{c}\text { Saturation } \\
\text { Pressure, } \\
P_{V} \\
(\mathrm{~Pa})\end{array}$ \\
\hline 527 & 216332997.3 & 483.8 & 0.000001006 & 51.03 & 0.00031558 & 0.0000107 & 0.3509 & 1136 & 0.957 \\
\hline 627 & 213884488 & 473.9 & 0.000001169 & 52.89 & 0.00028138 & 0.00001138 & 0.3373 & 1182 & 12.4 \\
\hline 727 & 211291948.7 & 463.9 & 0.00008213 & 54.54 & 0.0002547 & 0.0000119 & 0.3239 & 1230 & 96 \\
\hline 827 & 208699409.5 & 453.8 & 0.000401 & 55.99 & 0.0002334 & 0.00001227 & 0.3105 & 1279 & 509 \\
\hline 927 & 205962840.3 & 443.7 & 0.001494 & 57.24 & 0.00021598 & 0.00001253 & 0.2973 & 1327 & 2040 \\
\hline 1027 & 203226271.1 & 433.6 & 0.004319 & 58.29 & 0.00020143 & 0.00001271 & 0.2842 & 1374 & 6580 \\
\hline 1127 & 200345671.9 & 423.7 & 0.01162 & 59.13 & 0.00018907 & 0.00001284 & 0.2713 & 1420 & 17900 \\
\hline 1227 & 197609102.7 & 413.9 & 0.0262 & 59.78 & 0.00017844 & 0.00001296 & 0.2584 & 1464 & 42700 \\
\hline 1327 & 194584473.6 & 404.3 & 0.05314 & 60.22 & 0.00016918 & 0.00001307 & 0.2458 & 1508 & 91000 \\
\hline 1427 & 191559844.4 & 394.9 & 0.0987 & 60.46 & 0.00016102 & 0.0000132 & 0.2332 & 1550 & 177000 \\
\hline 1527 & 188535215.3 & 385.7 & 0.1701 & 60.5 & 0.00015379 & 0.00001333 & 0.2208 & 1592 & 319000 \\
\hline 1627 & 185366556.2 & 376.7 & 0.2578 & 60.33 & 0.00014731 & 0.00001348 & 0.2086 & 1633 & 540000 \\
\hline 1727 & 182053867.2 & 367.9 & 0.424 & 59.97 & 0.00014148 & 0.00001364 & 0.1965 & 1674 & 864000 \\
\hline
\end{tabular}




\section{Appendix C. Derivation of the vapor momentum equation (cylindrical pipe)}

A schematic illustration of the vapor flow in a constant area duct is shown in Figure C.1.

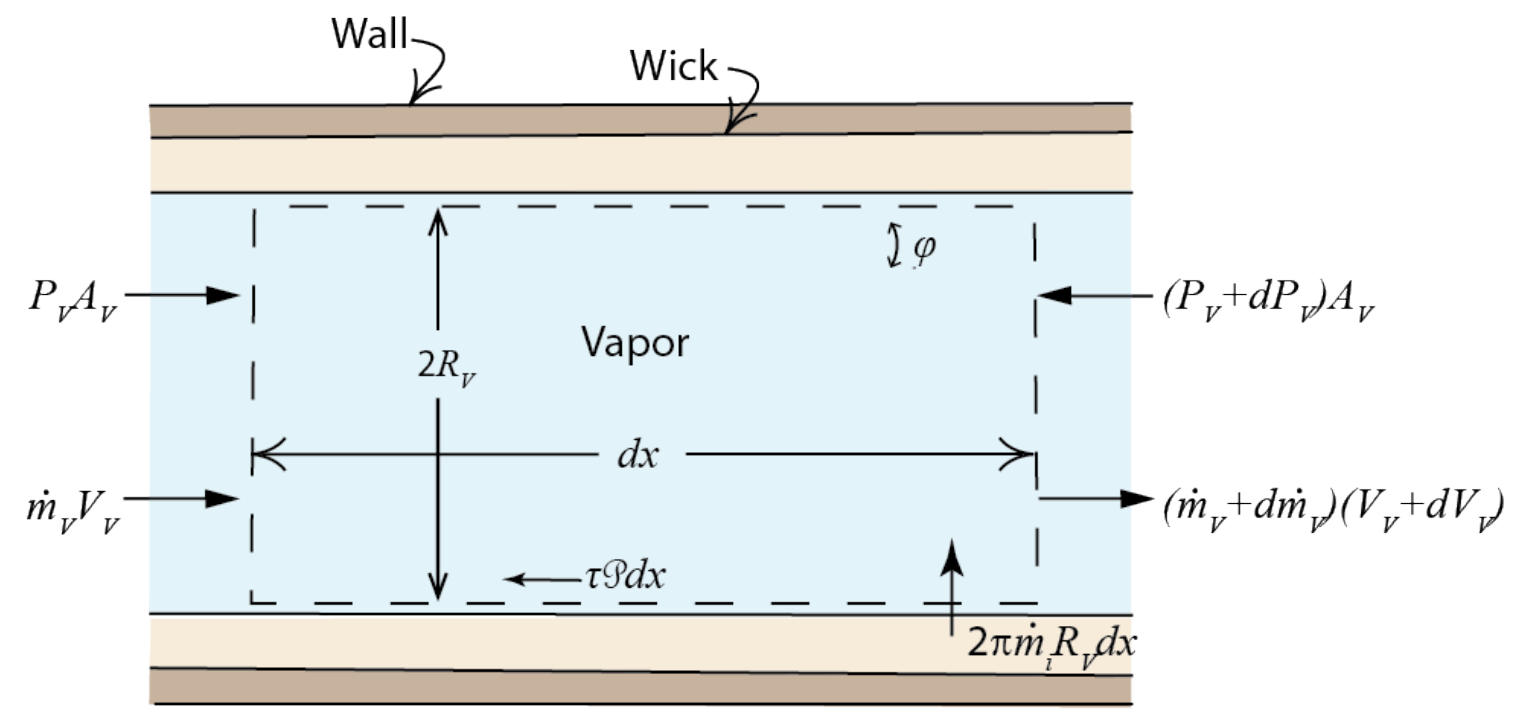

Figure C.1. Control volume for the vapor momentum equation of a constant area duct. 
A momentum balance over length $d x$ yields;

$$
\begin{aligned}
P_{V} A_{V}-\left(P_{V}+\right. & \left.d P_{V}\right) A_{V} \\
& =\beta\left[\left(\dot{m}_{V}+d \dot{m}_{V}\right)\left(V_{V}+d V_{V}\right)-\dot{m}_{V} d V_{V}\right]+\tau \mathcal{P} d x
\end{aligned}
$$

where $P_{V}$ is the vapor pressure, $A_{V}$ is the cross-sectional area, $\beta$ is the momentum correction parameter, $\dot{m}_{V}$ is the vapor mass flow rate, $V_{V}$ is the vapor velocity, and $\tau$ is the shear stress of the vapor flow at the walls, and $\mathcal{P}$ is the wetted perimeter of the duct, given by;

$$
\mathcal{P}=2 \pi R_{V}
$$

where $R_{V}$ is the vapor space radius. It has been assumed that the injected mass $\dot{m}_{i}$ is normal to the flow direction and does not contribute to the axial momentum. The shear stress on the walls is [145];

$$
\tau=\frac{f \rho_{V} V_{V}^{2}}{8}
$$

where $\rho_{V}$ is the vapor density and $f$ is the Darcy frictin factor.. Substituting (C.2) and (C.3) into (C.1);

$$
d P_{V}=-\frac{\beta}{A_{V}}\left(\dot{m}_{V} d V_{V}+V_{V} d \dot{m}_{V}\right)-\frac{f \rho_{V} V_{V}^{2} \pi R_{V}}{4 A_{V}} d x
$$

The vapor Reynolds number, $\operatorname{Re}_{V}$, is defined as; 


$$
\operatorname{Re}_{V}=\frac{\rho_{V} V_{V} D_{H}}{\mu_{V}}
$$

where $\mu_{V}$ is the vapor's viscosity and $D_{H}$ is the hydraulic diameter of the duct given by;

$$
D_{H}=2 R_{V}
$$

Substituting into equations (C.5) and (C.6) into (C.4), and noting the cross-sectional area $A_{V}=\pi R_{V}^{2}$, gives;

$$
d P_{V}=-\frac{\beta}{A_{V}}\left(\dot{m}_{V} d V_{V}+V_{V} d \dot{m}_{V}\right)-\frac{\mu_{V} f \operatorname{Re}_{V} V_{V}}{8 R_{V}^{2}} d x
$$

The vapor velocity can be expressed as a function of the mass flow rate, density, and area;'

$$
V_{V}=\frac{\dot{m}_{V}}{\rho_{V} A_{V}}
$$

Differentiating equation (C.8) gives;

$$
d V_{V}=\frac{1}{\rho_{V} A_{V}} d \dot{m}_{V}-\frac{\dot{m}_{V}}{\rho_{V}^{2} A_{V}} d \rho_{V}
$$

Equations (C.8) and (C.9) can be used to express equation (C.7) as;

$$
d P_{V}=-\frac{\beta}{\rho_{V} A_{V}^{2}}\left(2 \dot{m}_{V} d \dot{m}_{V}-\frac{\dot{m}_{V}^{2}}{\rho_{V}} d \rho_{V}\right)-\frac{f \operatorname{Re}_{V} \mu_{V} \dot{m}_{V}}{8 A_{V} R_{V}^{2} \rho_{V}} d x
$$

The mass flow rate $\dot{m}_{V}$ is a function of the vapor flow's heat transport rate, $Q$; 


$$
\dot{m}_{V}=\frac{Q}{\lambda}
$$

where $\lambda$ is the latent heat of vaporization. Differentiating gives;

$$
d \dot{m}_{V}=\frac{1}{\lambda} d Q
$$

Substituting equations (C.11) and (C.12) into (C.10) completes the expression for the pressure drop across $d x$ as a function of the heat transport rate, $Q$, for a cylinder;

$$
d P_{V}=-\frac{\beta}{\rho_{V} A_{V}^{2} \lambda^{2}}\left(2 Q d Q-\frac{Q^{2}}{\rho_{V}} d \rho_{V}\right)-\frac{f \operatorname{Re}_{V} \mu_{V}}{8 A_{V} R_{V}{ }^{2} \rho_{V} \lambda} Q d x
$$




\section{Appendix D. Derivation of the vapor momentum equation (wedge geometry)}

A schematic illustration of the vapor flow in a diverging wedge is shown in Figure D.1.

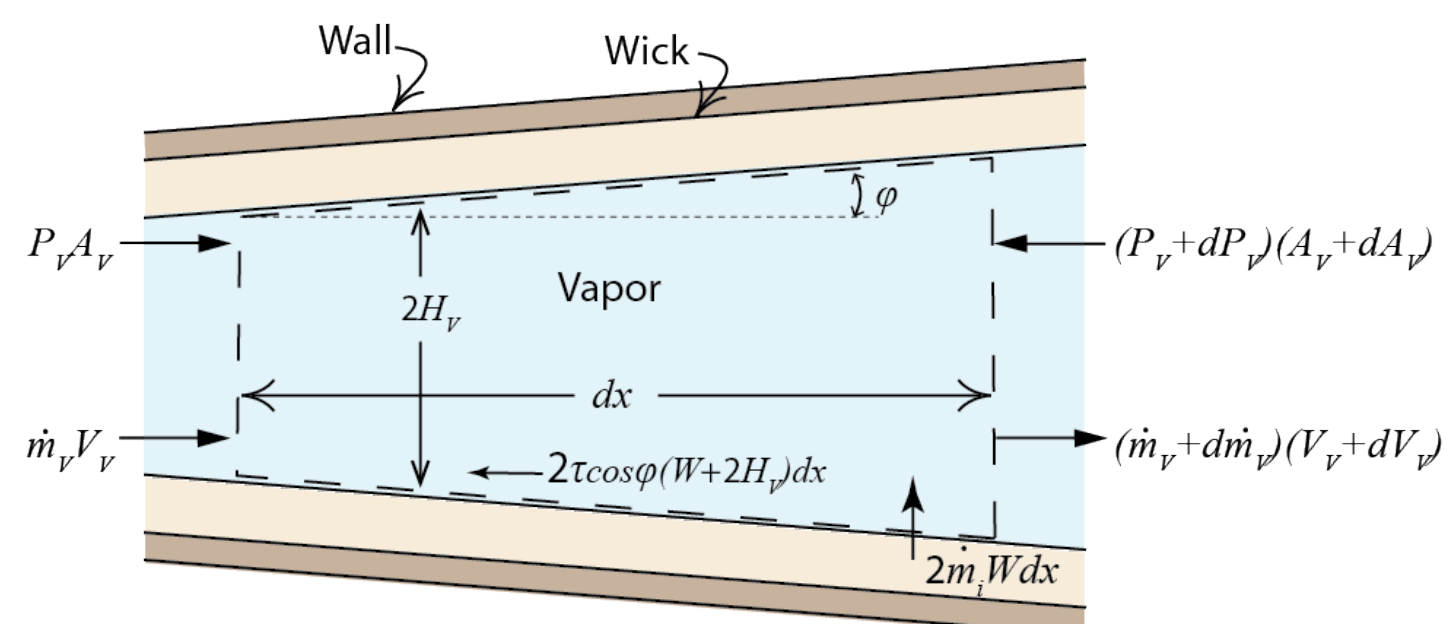

Figure D.1. Control volume for the vapor momentum equation of a diverging duct. 
A momentum balance over length $d x$ yields;

$$
\begin{aligned}
P_{V} A_{V}-\left(P_{V}+\right. & \left.d P_{V}\right)\left(A_{V}+d A_{V}\right)+\left(P_{V}+\frac{d P_{V}}{2}\right) d A_{V} \\
= & \beta\left[\left(\dot{m}_{V}+d \dot{m}_{V}\right)\left(V_{V}+d V_{V}\right)-\dot{m}_{V} V_{V}\right] \\
& +2 \tau\left(W+2 H_{V}\right) \cos \varphi d x
\end{aligned}
$$

where $P_{V}$ is the vapor pressure, $A_{V}$ is the cross-sectional area, $\beta$ is the momentum correction parameter, $\dot{m}_{V}$ is the vapor mass flow rate, $V_{V}$ is the vapor velocity, and $\tau$ is the shear stress of the vapor flow at the walls. It has been assumed that the injected mass $\dot{m}_{i}$ is normal to the flow direction and does not contribute to the axial momentum. The cross-sectional area and shear stress [145] are given by;

$$
A_{V}=2 H_{V} W
$$

and

$$
\tau=\frac{f \rho_{V} V_{V}^{2}}{8}
$$

where $\rho_{V}$ is the vapor density and $f$ is the Darcy frictin factor.. Substituting equations (D.2) and (D.3) into (D.1); 


$$
\begin{aligned}
d P_{V}=-\frac{\beta}{A_{V}}\left(\dot{m}_{V} d V_{V}+V_{V} d \dot{m}_{V}\right) & \\
& -\frac{f \rho_{V} V_{V}^{2}\left(W+2 H_{V}\right) \cos \varphi}{4 A_{V}} d x
\end{aligned}
$$

The vapor Reynolds number, $\operatorname{Re}_{V}$, is defined as;

$$
\operatorname{Re}_{V}=\frac{\rho_{V} V_{V} D_{H}}{\mu_{V}}
$$

where $\mu_{V}$ is the vapor's viscosity and $D_{H}$ is the hydraulic diameter of the wedge given by;

$$
D_{H}=\frac{A_{V}}{8\left(W+2 H_{V}\right)}
$$

Substituting into equations (D.5) and (D.6) into (D.4) produces;

$$
\begin{aligned}
d P_{V}=-\frac{\beta}{A_{V}}\left(\dot{m}_{V} d V_{V}+V_{V} d \dot{m}_{V}\right) & \\
& -\frac{2 \mu_{V} f \operatorname{Re}_{V}\left(W+2 H_{V}\right)^{2}}{A_{V}{ }^{2}} V_{V} \cos \varphi d x
\end{aligned}
$$

The vapor velocity can be expressed as a function of the mass flow rate, density, and area;'

$$
V_{V}=\frac{\dot{m}_{V}}{\rho_{V} A_{V}}
$$

Differentiating equation (D.8) gives; 


$$
d V_{V}=\frac{1}{\rho_{V} A_{V}} d \dot{m}_{V}-\frac{\dot{m}_{V}}{\rho_{V}^{2} A_{V}} d \rho_{V}-\frac{\dot{m}_{V}}{\rho_{V} A_{V}^{2}} d A_{V}
$$

which can be used to express equation (D.7) as;

$$
\begin{array}{r}
d P_{V}=-\frac{\beta}{\rho_{V} A_{V}^{2}}\left(2 \dot{m}_{V} d \dot{m}_{V}-\frac{\dot{m}_{V}^{2}}{\rho_{V}} d \rho_{V}-\frac{\dot{m}_{V}^{2}}{A_{V}} d A_{V}\right) \\
-\frac{2 \mu_{V} f \operatorname{Re}_{V}\left(W+2 H_{V}\right)^{2} \cos \varphi}{\rho_{V} A_{V}{ }^{3}} \dot{m}_{V} d x
\end{array}
$$

The mass flow rate $\dot{m}_{V}$ can be expressed as a function of the vapor flow's heat transport rate, $Q$

$$
\dot{m}_{V}=\frac{Q}{\lambda}
$$

where $\lambda$ is the latent heat of vaporization. Differentiating gives;

$$
d \dot{m}_{V}=\frac{1}{\lambda} d Q
$$

Substituting equations (D.11) and (D.12) into (D.10) completes the expression for the pressure drop across $d x$ as a function of the heat transport rate, $Q$, for a wedge;

$$
\begin{array}{r}
d P_{V}=-\frac{\beta}{\rho_{V} A_{V}{ }^{2} \lambda^{2}}\left(2 Q d Q-\frac{Q^{2}}{\rho_{V}} d \rho_{V}-\frac{Q^{2}}{A_{V}} d A_{V}\right) \\
-\frac{2 \mu_{V} f \operatorname{Re}_{V}\left(W+2 H_{V}\right)^{2} \cos \varphi}{\lambda \rho_{V} A_{V}{ }^{3}} Q d x
\end{array}
$$




\section{Appendix E. Error analysis}

The methodology used for error analysis is described in [146].

\section{E.1 Infrared temperature measurements}

Temperature measurements taken using the infrared camera are adjusted for two known sources of error: random error, $u_{r}$, and camera instrumentation error, $u_{c}$. The random error is determined by computing the standard deviation of the assumed temperature value over a two hour period for the low temperature testing and over a period of five minutes for the high temperature testing. The instrumentation error is listed in the product literature and includes calibration error. Table E. 1 shows the values for these errors. 
Table E.1. Infrared temperature uncertainty.

\begin{tabular}{ccc}
\hline \hline Uncertainty & Details & Value \\
\hline$u_{r}$ & Random error taken as std. dev. & $0.05^{\circ} \mathrm{C}$ (average) \\
& & \\
$u_{c}$ & Instrumentation error & greater of $\pm 2^{\circ} \mathrm{C}$ or \\
& & $\pm 2 \%$ of reading \\
\hline \hline
\end{tabular}

The total error, $u_{I R}$, is calculated as;

$$
u_{I R}^{2}=u_{r}^{2}+u_{c}^{2}
$$

\section{E.2 Applied power}

Testing of the low temperature, SS304/H2O system used resistance thin film heaters to deliver heat to the leading edge tip. The applied power, $Q_{A}$ was calculated from a measurement of the circuit resistance, $R$, and voltage, $V$, using;

$$
Q_{A}=V^{2} / R
$$

Using the root-sum-squared uncertainty method, the error propagation for the applied power, $u_{Q_{A}}$, is calculated from the individual uncertainties;

$$
u_{Q_{A}}{ }^{2}=\left(\frac{\partial Q_{A}}{\partial V} u_{V}\right)^{2}+\left(\frac{\partial Q_{A}}{\partial R} u_{R}\right)^{2}
$$

where $u_{V}$ and $u_{R}$ are the uncertainty of the voltage and resistance measurement, respectively. Taking the derivative of equation (E.2) and substituting into (E.3), the error propagation becomes; 


$$
u_{Q_{A}}{ }^{2}=\left(\frac{2 V}{R} u_{V}\right)^{2}+\left(\frac{V^{2}}{R^{2}} u_{R}\right)^{2}
$$

The error sources for $u_{V}$ and $u_{R}$ are given in Table E.2.

Table E.2. Uncertainties associated with the applied power.

\begin{tabular}{ccc}
\hline \hline Uncertainty & Details & Value \\
\hline$u_{\text {variac }}$ & Variable transformer uncertainty. Taken as smallest & possible voltage change \\
$u_{m m, V}$ & $\begin{array}{c}\text { Multimeter instrumentation uncertainty operating in } \\
\text { voltmeter mode }\end{array}$ & $\pm 0.15 \mathrm{~V}$ \\
$u_{m m, R}$ & $\begin{array}{c}\text { Multimeter instrumentation uncertainity operating in } \\
\text { ohmmeter mode }\end{array}$ & $\pm 0.8 \%$ reading[147] \\
\hline \hline
\end{tabular}

The total errors are then calculated as;

$$
u_{V}^{2}=u_{\text {variac }}^{2}+u_{m m, V}^{2}
$$

$$
u_{R}^{2}=u_{m m, R}^{2}
$$

\section{E.3 Temperature Difference Calculations, $\Delta T_{s}$}

The temperature difference across the samples is calculated using;

$$
\Delta T_{\boldsymbol{s}}=T_{s, x=0}-T_{s, x=L}
$$

The root-sum-squared uncertainty is calculated by;

$$
u_{\Delta T}^{2}=u_{T}^{2}+u_{I R}^{2}
$$


For the low temperature experiments, $T_{s, x=0}$ is taken by thermocouple measurement and is associated with error $u_{T}=u_{T / C}$. For the high temperature experiments, $T_{s, x=0}$ is taken by IR with $u_{T}=u_{I R}$. For both sets of experiments, $T_{s, x=L}$ is taken by IR and is associated with errors noted by $u_{I R}$ in (E.1). Experimental errors are shown in Table E.3.

Table E.3. Uncertainties associated with the temperature difference calculation.

\begin{tabular}{ccc}
\hline \hline Uncertainty & Details & Value \\
\hline$u_{r}$ & Random error taken as std. dev. & $0.05^{\circ} \mathrm{C}$ (average) \\
$u_{c}$ & Instrumentation error & greater of $\pm 2^{\circ} \mathrm{C}$ or \\
& & $\pm 2 \%$ of reading \\
$u_{T / C}$ & Error for Type K thermocouple & $\pm 1.1^{\circ} \mathrm{C}[148]$ \\
\hline \hline
\end{tabular}

\section{E.4 Thermal Stress Calculations}

The thermal stress is;

$$
\sigma_{t h}=\frac{E \alpha}{1-v}\left(T_{\max }-T_{V}\right)
$$

Values $E, \alpha$, and $v$ are literature values and taken as exact. The root-sum-squared uncertainty is calculated by;

$$
u_{\sigma_{t h}}{ }^{2}=\left(\frac{\partial \sigma_{t h}}{\partial T_{\max }} u_{T / C}\right)^{2}+\left(\frac{\partial \sigma_{t h}}{\partial T_{V}} u_{I R}\right)^{2}
$$

where;

$$
\frac{\partial \sigma_{t h}}{\partial T_{\max }}=\frac{E \alpha}{1-v}
$$

and, 


$$
\frac{\partial \sigma_{t h}}{\partial T_{V}}=-\frac{E \alpha}{1-v}
$$

$T_{\max }$ is taken by thermocouple measurement and is associated with error $u_{T / C} \cdot T_{V}$ is taken by IR and is associated with errors noted by equation (E.1). Experimental errors are shown in Table E.4.

Table E.4 Uncertainties associated with the thermal stress calculation.

\begin{tabular}{ccc}
\hline \hline Uncertainty & Details & Value \\
\hline$u_{r}$ & Random error taken as std. dev. & $0.05^{\circ} \mathrm{C}$ (average) \\
$u_{c}$ & Instrumentation error & greater of $\pm 2^{\circ} \mathrm{C}$ or \\
$u_{T / C}$ & Error for Type K thermocouple & $\pm 2 \%$ of reading \\
\hline \hline
\end{tabular}

